\title{
CP VIOLATION IN SINGLE TOP QUARK PRODUCTION \\ By
}

Weigang Geng

\section{A DISSERTATION}

Submitted to

Michigan State University

in partial fulfillment of the requirements

for the degree of

\section{DOCTOR OF PHILOSOPHY}

Physics

2012 
UMI Number: 3521592

All rights reserved

INFORMATION TO ALL USERS

The quality of this reproduction is dependent on the quality of the copy submitted.

In the unlikely event that the author did not send a complete manuscript and there are missing pages, these will be noted. Also, if material had to be removed, a note will indicate the deletion.

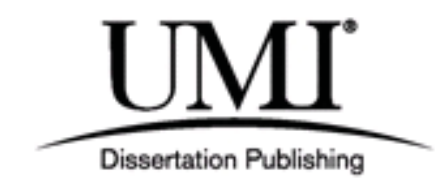

UMI 3521592

Copyright 2012 by ProQuest LLC.

All rights reserved. This edition of the work is protected against unauthorized copying under Title 17, United States Code.

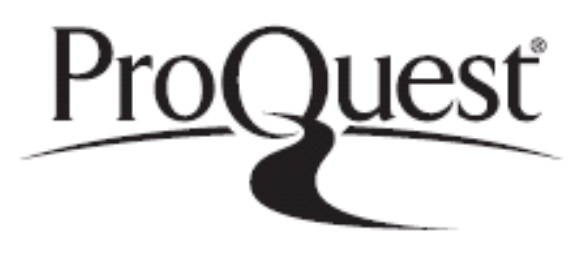

ProQuest LLC.

789 East Eisenhower Parkway

P.O. Box 1346

Ann Arbor, MI 48106 - 1346 


\section{ABSTRACT CP VIOLATION IN SINGLE TOP QUARK PRODUCTION \\ By \\ Weigang Geng}

We present a search for CP violation in single top quark production with the $D \varnothing$ experiment at the Tevatron proton-antiproton collider. CP violation in the top electroweak interaction results in different single top quark production cross sections for top and antitop quarks. We perform the search in the single top quark final state using $5.4 \mathrm{fb}^{-1}$ of data, in the $s$-channel, $t$-channel, and for both combined. At this time, we do not see an observable CP asymmetry. 
To Ni. 


\section{TABLE OF CONTENTS}

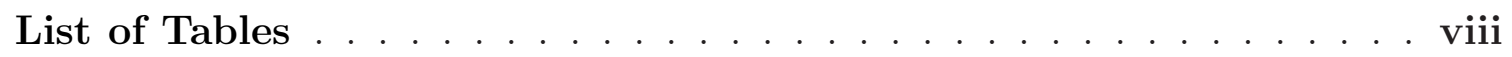

List of Figures . . . . . . . . . . . . . . . . . . . $\quad$ x

Chapter 1 Introduction $\ldots \ldots \ldots \ldots \ldots$

1.1 CP Violation . . . . . . . . . . . . . . . . . . 2

1.2 Motivation for searching for $\mathrm{CP}$ violation in single top . . . . . . 3

1.3 Samples selection and single top analysis overview . . . . . . . . 4

Chapter 2 Theory . . . . . . . . . . . . . . . . . . 6

2.1 The Standard Model . . . . . . . . . . . . . . . . . . . . . . . 7

2.1 .1 Fermions . . . . . . . . . . . . . . . . . . . . . . . 9

2.1.2 Gauge Bosons . . . . . . . . . . . . . . . . . . . . 11

2.1 .3 Higgs Boson . . . . . . . . . . . . . . . . . . . . . . 12

2.1.4 Limits of the Standard Model . . . . . . . . . . . . . . 13

2.2 The Top Quark . . . . . . . . . . . . . . . . . . . . . . 14

2.2 .1 Properties . . . . . . . . . . . . . . . . . . . . . . 14

2.2 .2 Production . . . . . . . . . . . . . . . . . . . . 15

2.2 .3 Decay . . . . . . . . . . . . . . . . . . . 15

2.3 The single top quark . . . . . . . . . . . . . . . . . 16

2.3.1 Single top production and decay at the Tevatron . . . . . . 16

2.3.2 Motivations for searching for CP Violation in single top . . . . 26

2.4 Outline of CP violation measurement in single top . . . . . . . . . 28

Chapter 3 Experimental Setup . . . . . . . . . . . . . . . 30

3.1 The Accelerator Complex and Tevatron . . . . . . . . . . . . . 31

3.2 Luminosity and Cross Sections . . . . . . . . . . . . . . 35 
3.3 The $D \varnothing$ detector $\ldots \ldots \ldots \ldots$

3.3.1 Particle Signatures . . . . . . . . . . . . . . . . . . 40

3.3.2 Coordinate System and Pseudorapidity . . . . . . . . . . . 43

3.3.3 The Central Tracking System . . . . . . . . . . . . . . . 44

3.3.3.1 Silicon Micro-strip Tracker (SMT) _. . . . . . . 46

3.3.3.2 Central Fiber Tracker (CFT) . . . . . . . . . . 46

3.3.4 The Central and Forward Preshower Detectors . . . . . . . . 47

3.3.5 The Calorimeters and ICD . . . . . . . . . . . . . . 48

3.3.5.1 Calorimeters . . . . . . . . . . . . . 48

3.3.5.2 Intercryostat Detector . . . . . . . . . . . . . 51

3.3.6 The Muon System . . . . . . . . . . . . . . . . . . . . . 51

3.3.7 The Luminosity Monitor . . . . . . . . . . . . . . . . . . . 52

3.3.8 The DØ Trigger System . . . . . . . . . . . . . . . . . 53

3.3.8.1 The Level 1 Trigger . . . . . . . . . . . . . . 54

3.3.8.2 The Level 2 Trigger . . . . . . . . . . . . . . 55

3.3.8.3 The Level 3 Trigger . . . . . . . . . . . . . . 58

Chapter 4 Event Reconstruction and Object Identification . . . . 60

4.1 Tracks . . . . . . . . . . . . . . . . . . . . . . . . 61

4.2 Primary Vertices . . . . . . . . . . . . . . . . . 63

4.3 Electrons. . . . . . . . . . . . . . . . . . . 65

4.4 Muons . . . . . . . . . . . . . . . . . . . . . . . . 66

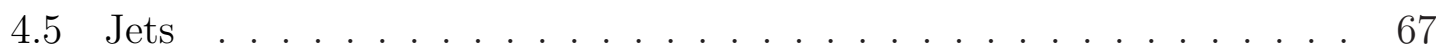

$4.6 \quad$ b Jets . . . . . . . . . . . . . . . . . . . . . . . . . 69

4.7 Missing Transverse Energy . . . . . . . . . . . . . . . . . 71

Chapter 5 Event Selection . . . . . . . . . . . . . . . 73

5.1 Strategy . . . . . . . . . . . . . . . . . . . . . 73

5.2 Event selection cuts . . . . . . . . . . . . . . . . . 74

5.2 .1 General selection . . . . . . . . . . . . . . . . . 74

5.2 .2 Electron channel selection $\ldots \ldots \ldots \ldots \ldots$

5.2 .3 Muon channel selection . . . . . . . . . . . . . . . . . . . 77

$5.2 .4 \quad b$-tagging selection $\ldots \ldots \ldots \ldots \ldots$

Chapter 6 Data and Simulation Samples . . . . . . . . . . . . 80

6.1 Data samples . . . . . . . . . . . . . . . . . . . . . . 80

6.1.1 Signal data samples . . . . . . . . . . . . . . . . . . 80

6.1 .2 Multijets data samples . . . . . . . . . . . . . . 81 
6.1.2.1 Multijets in the electron channel . . . . . . . . . . 82

6.1.2.2 Multijets in the muon channel . . . . . . . . . . 83

6.2 Simulation samples . . . . . . . . . . . . . . . . . . 83

6.2.1 Signal simulation samples . . . . . . . . . . . . . 83

6.2.2 Background simulation samples . . . . . . . . . . . . 84

6.2.3 Simulation samples corrections and normalization . . . . . . . 84

6.2.3.1 Simulation efficiencies corrections . . . . . . . . 86

6.2.3.2 $W+$ jets and multijets backgrounds . . . . . . . . 88

6.3 Lepton charge mis-ID measurement samples . . . . . . . . . . . . . . 89

Chapter 7 Event Yields and Data/Simulation Comparison . . . . 91

7.1 Event yields . . . . . . . . . . . . . . . . . . 91

7.2 Signal acceptance . . . . . . . . . . . . . . . . . . . . . . . . . . . . . . . . . . . . . . . . . . . . . . . . . . .

7.3 Data and Monte Carlo comparison . . . . . . . . . . . . . . . 99

Chapter 8 Multivariate Analysis . . . . . . . . . . . . . . . . 104

8.1 Split samples . . . . . . . . . . . . . . . . . . . 105

8.2 Output transformation . . . . . . . . . . . . . . . . 107

8.3 Key for plots . . . . . . . . . . . . . . . . . . . . . . . . 109

8.4 Boosted Decision Trees (BDT) . . . . . . . . . . . . . . 110

8.5 Bayesian Neural Networks (BNN) . . . . . . . . . . . . . . 115

8.6 Neuroevolution of augmented topologies (NEAT) . . . . . . . . . . 120

8.7 A combined BNN using BDT, BNN and NEAT discriminants . . . . 125

Chapter 9 Statistical Analysis . . . . . . . . . . . . 132

9.1 Poisson distribution likelihood . . . . . . . . . . . . . . . . . . . 132

9.2 Bayesian analysis . . . . . . . . . . . . . . . . . 134

9.3 Posterior probability density for the CP asymmetry . . . . . . . . . . 140

Chapter 10 Systematic Uncertainties . . . . . . . . . . . . . . . 142

10.1 Uncertainties affecting normalization only . . . . . . . . . . . . 143

10.2 Uncertainties affecting normalization and shape . . . . . . . . . 150

Chapter 11 CP Violation Measurement . . . . . . . . . . . 153

11.1 Cross section measurement . . . . . . . . . . . . . . . 153

11.2 Expected results . . . . . . . . . . . . . . . . . . . 157

11.3 Observed results . . . . . . . . . . . . . . . . . 162 
Chapter 12 Results and Conclusions . . . . . . . . . . . . . 167

Appendix A Plots After Splitting by the Lepton Charge . . . . . . 169

Appendix B SAM Definitions of Dataset Used in This Analysis . . . 183

Appendix C Plots for discriminants after the binning transformation 185

Appendix D Lepton Charge Mis-Identification . . . . . . . . . . 190

D.1 Electron Charge Mis-ID Rate . . . . . . . . . . . . . . . . 193

D.2 Muon Charge Mis-ID Rate . . . . . . . . . . . . . . . 195

D.3 Conclusion . . . . . . . . . . . . . . . . . . . . . . . . . . . . . . 198

Appendix E W Asymmetry Check . . . . . . . . . . . . . . 199

Appendix F $b / \bar{b}$ Jet Energy Scale (JES) Check . . . . . . . . . . 202

Appendix G Production and decay asymmetries . . . . . . . . 212

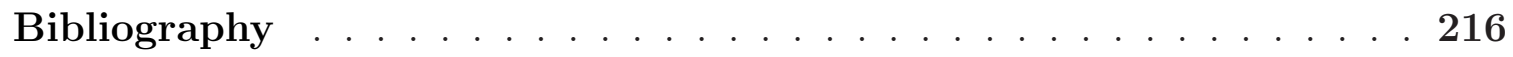




\section{LIST OF TABLES}

Table $2.1 \quad$ Standard Model gauge bosons . . . . . . . . . . . . . . . . . 12

Table 2.2 Single top quark production cross sections [1]. . . . . . . . . 17

Table 6.1 Integrated luminosity for the datasets used in this analysis. . 81

Table 6.2 The Monte Carlo samples event samples . . . . . . . . . . . . 85

Table 7.1 One $b$ tagged event yields . . . . . . . . . . . . . . 94

Table 7.2 Two $b$ tagged event yields . . . . . . . . . . . . . . . . . . 95

Table 7.3 Expected and observed yields in "positive" samples . . . . . 96

Table 7.4 Expected and observed yields in "negative" samples . . . . . 97

Table 7.5 Combined (Electron+Muon, Run IIa+Run IIb) signal acceptances. 99

Table 8.1 BDT discriminant used in the current analysis. . . . . . . . . 112

Table 11.1 Expected results for CP asymmetry in s, t, s+t channels. . . 161

Table 11.2 Observed results for CP asymmetry in s, t, s+t channels. . . 166

Table D.1 Overall Charge mis-ID Rates. . . . . . . . . . . . . . . 198 viii 
Table F.1 Event yields for signals in "positive" samples . . . . . . . . . 203

Table F.2 Event yields for signals in "positive" samples b/bbar JES . . 203

Table F.3 Event yields for signals in "negative" samples . . . . . . . . . 204

Table F.4 Event yields for signals in "negative" samples b/bbar JES . . 204

Table F.5 Ratio of integrals for variables before and after b/ $/ \bar{b}$ JES . . . 205 


\section{LIST OF FIGURES}

Figure 1.1 Flow chart of the stages of the analysis overview . . . . . . 5

Figure 2.1 The Standard Model of elementary particles . . . . . . . . 8

Figure 2.2 Feynman diagram for the quark-antiquark annihilation . . . 16

Figure 2.3 Feynman diagram for single top s-channel production. . . . . 18

Figure $2.4 \quad$ Feynman diagram for single top $t$-channel production. . . . . 19

Figure 2.5 Decay branching ratio of a top quark . . . . . . . . . . 20

Figure $2.6 \quad$ Feynman diagram for single top $s$-channel . . . . . . . . . . 20

Figure $2.7 \quad$ Feynman diagram for single top $t$-channel . . . . . . . . . . . 21

Figure 2.8 The transverse momentum for $s$-channel . . . . . . . . . . . . 22

Figure 2.9 The transverse momentum for $t$-channel . . . . . . . . . . . 23

Figure 2.10 The pseudorapidity for $s$-channel . . . . . . . . . . . . 24

Figure 2.11 The pseudorapidity for $t$-channel . . . . . . . . . . . . 25

Figure 2.12 Feynman diagram for $W^{\prime}$ boson decaying into single top. . . 27 
Figure 2.13 FCNC via exchange of a gluon in single top production. . . . 28

Figure $3.1 \quad$ Fermilab's accelerater chain. . . . . . . . . . . . . . . . . . 32

Figure 3.2 Fermilab's Tevatron Tunnel . . . . . . . . . . . . . . . 36

Figure 3.3 Side view of the $D \varnothing$ detector . . . . . . . . . . . . . . . . 39

Figure $3.4 \quad$ A schematic of a hermetic detector . . . . . . . . . . . . 41

Figure 3.5 High energy particle signatures left in the detector components 42

Figure 3.6 The Tracking System at DØ. . . . . . . . . . . . . . . . 45

Figure 3.7 The disk/barrel design of the silicon microstrip tracker. . . . 47

Figure $3.8 \quad$ Illustration of the calorimeter systems. . . . . . . . . . . . . . 49

Figure 3.9 Intersecting view of a quarter of the $D \varnothing$ calorimeter . . . . 50

Figure 3.10 Trigger-related data flow in L1 and L2 trigger systems. . . . 58

Figure $4.1 \quad$ Illustration of a primary vertex. . . . . . . . . . . . . . 64

Figure 4.2 Illustration of jet formation. . . . . . . . . . . . . . . . 68

Figure 4.3 Illustration of a secondary vertex . . . . . . . . . . . . 70

Figure 7.1 A pie chart illustration of backgrounds and signals . . . . . . 92

Figure 7.2 Lepton transverse momentum . . . . . . . . . . . . . . 100

Figure 7.3 HT for positive and negative samples . . . . . . . . . . . 101

Figure 7.4 The Lepton Eta . . . . . . . . . . . . . . . . . 101 
Figure 7.5 Single top mass distribution . . . . . . . . . . . . . 102

Figure 7.6 Top Mass distribution for positive and negative samples . . . 102

Figure 7.7 W Transverse mass distribution . . . . . . . . . 103

Figure 8.1 Flowcharts of the MVA analyses. . . . . . . . . . 106

Figure 8.2 Color scheme . . . . . . . . . . . . . . . . . . . . . . . . . . . 109

Figure 8.3 Decision tree illustration . . . . . . . . . . . . . 111

Figure $8.4 \quad$ BDT output for $s+t$ channel $\ldots \ldots \ldots \ldots$

Figure 8.5 BDT output for $s$-channel . . . . . . . . . . . . 114

Figure 8.6 BDT output for $t$-channel . . . . . . . . . . . . . . 115

Figure 8.7 An illustration of a simple Neural Network. . . . . . . . . 116

Figure $8.8 \quad$ BNN output for $s+t$ channel $\ldots \ldots \ldots \ldots \ldots$

Figure $8.9 \quad$ BNN output for $s$ channel $\ldots \ldots \ldots \ldots$

Figure 8.10 BNN output for t channel . . . . . . . . . . . . . . . 120

Figure 8.11 NEAT output for $s+t$ channel $\ldots \ldots \ldots \ldots \ldots$

Figure 8.12 NEAT output for $s$ channel . . . . . . . . . . . . . . 123

Figure 8.13 NEAT output for t channel . . . . . . . . . . . . . . . . 124

Figure 8.14 Combined BNN output for $s+t$ channel $\ldots \ldots \ldots \ldots$

Figure 8.15 Combined BNN output for $s$ channel . . . . . . . . . 127 
Figure 8.16 Combined BNN output for t channel . . . . . . . . . . . . . . 128

Figure $8.17 \quad s+t$ channel discriminant plots . . . . . . . . . . . . . . 129

Figure 8.18 Zoomed $s+t$ channel discriminant plots . . . . . . . . . . . 129

Figure $8.19 t$ channel discriminant plots . . . . . . . . . . . . . . . 130

Figure 8.20 Zoomed $t$ channel discriminant plots . . . . . . . . . . . . . 130

Figure $8.21 s$ channel discriminant plots . . . . . . . . . . . . 131

Figure 8.22 Zoomed $s$ channel discriminant plots . . . . . . . . . . . 131

Figure $9.1 \quad$ Illustration of flat probability density . . . . . . . . . . . 135

Figure 9.2 Illustration of cross section measurement . . . . . . . . . 136

Figure 9.3 Illustration of two dimensional posterior probability density . 139

Figure 11.1 Illustration of a $2 d$ posterior . . . . . . . . . . . . . . . 155

Figure 11.2 Illustration of measuring CP violation . . . . . . . . . . . 156

Figure 11.3 Expected CP Violation results in $s+t$-channel . . . . . . . . 158

Figure 11.4 Expected CP Violation results in $t$-channel . . . . . . . . . . 159

Figure 11.5 Expected CP Violation results in s-channel . . . . . . . . . . 160

Figure 11.6 Observed CP Violation results in $s+t$-channel . . . . . . . . 163

Figure 11.7 Observed CP Violation results in $t$-channel . . . . . . . . . . 164

Figure 11.8 Observed CP Violation results in $s$-channel . . . . . . . . . . 165 xiii 
Figure A.1 Color scheme . . . . . . . . . . . . . . . . . . . 170

Figure A.2 Transverse momentum of lepton for both samples . . . . . 171

Figure A.3 Centrality for All Jets for both samples . . . . . . . . . . 171

Figure A.4 Delta Phi between Lepton MET for both samples . . . . . 172

Figure A.5 HT for both samples . . . . . . . . . . . . . . . 172

Figure A.6 HT-AllJets-MinusBTaggedJet for both samples . . . . . . . 173

Figure A.7 Invariant Mass of two leading jets for both samples . . . . . 173

Figure A.8 InvariantMass-LightQuarkJets1-2 for both samples . . . . . 174

Figure A.9 Leading b Tagged Jet for both samples . . . . . . . . . . 174

Figure A.10 LeadingBTaggedJetEta for both samples . . . . . . . . 175

Figure A.11 LeadingBTaggedJetLeptonDeltaPhi for both samples . . . . . 175

Figure A.12 LeadingBTaggedJetPt for both samples . . . . . . . . . 176

Figure A.13 LeadingLightQuarkJetBTagNN for both samples . . . . . 176

Figure A.14 LeadingLightQuarkJetEta for both samples . . . . . . . 177

Figure A.15 LeadingLightQuarkJetLeptonDeltaPhi for both samples . . . 177

Figure A.16 LeadingLightQuarkJetPt for both samples . . . . . . 178

Figure A.17 LeptonEta for both samples _. . . . . . . . . . 178

Figure A.18 METPt for both samples _ . . . . . . . . . . . . . 179 
Figure A.19 QTimesEta for positive and negative samples . . . . . . . 179

Figure A.20 SecondLightQuarkJetPt for positive and negative samples . . 180

Figure A.21 Single top mass for positive and negative samples $\ldots \ldots$. 180

Figure A.22 SpinCorr for positive and negative samples . . . . . . . . 181

Figure A.23 TopMassMinChiSqr for positive and negative samples . . . . 181

Figure A.24 WT for positive and negative samples . . . . . . . 182

Figure C.1 $\quad s+t$ channel discriminant plots . . . . . . . . . 187

Figure C.2 Zoomed $s+t$ channel discriminant plots . . . . . . 187

Figure C.3 $\quad t$ channel discriminant plots . . . . . . . . . . . 188

Figure C.4 Zoomed $t$ channel discriminant plots . . . . . . . 188

Figure C.5 $\quad s$ channel discriminant plots for $\ldots \ldots \ldots \ldots$

Figure C.6 Zoomed $s$ channel discriminant plots . . . . . . . . . . 189

Figure D.1 Muon charge mis-ID rate as a function of $p_{T} \ldots \ldots \ldots 197$

Figure E.1 Expected posterior density for $W+$ jets asymmetry $\ldots . .200$

Figure E.2 Observed posterior density for $W+$ jets asymmetry . . . . 201

Figure F.1 HT for all jets before and after $\mathrm{b} / \bar{b}$ JES $\ldots \ldots \ldots 206$

Figure F.2 Leading jet transverse momentum before and after b/ $/ \bar{b}$ JES 207

Figure F.3 Single top mass before and after b/ $/ \bar{b}$ JES $\ldots \ldots \ldots . . .208$ 
Figure F.4 HT for all jets before and after b/b JES . . . . . . . . . . 209

Figure F.5 Leading jet transverse momentum before and after b/ $\bar{b}$ JES 210

Figure F.6 Single top mass before and after b/ JES . . . . . . . . . . . 211 


\section{Chapter 1}

\section{Introduction}

This thesis gives an overview of the first search for $\mathrm{CP}$ violation in single top quark production at the $D \emptyset$ experiment at the Fermilab Tevatron Collider. Chapter 1 gives an introduction to $\mathrm{CP}$ violation and the motivation for this search in the single top process; Chapter 2 sketches the theory background; Chapter 3 gives an introduction of the experimental facilities used in this analysis; Chapter 4 describes how to identify basic physics objects; Chapter 5 presents the data and simulation samples used in this analysis; Chapter 6 shows the event selection cuts and describes how the background samples are normalized; Chapter 7 presents the multivariate methods used; Chapter 8 describes how the statistical methods are implemented; Chapter 9 discusses systematic uncertainties encountered in this analysis; Chapter 10 shows the specific methods used to measure the $\mathrm{CP}$ asymmetry and the results; Chapter 11 
concludes this thesis by summarizing the results of the analysis and giving an outlook for future analyses.

\subsection{CP Violation}

Charge conjugation and Parity (CP) symmetry states that the laws of physics should be the same if a particle were interchanged with its antiparticle ( $C$ symmetry), and left and right were swapped ( $P$ symmetry). CP-symmetry was proposed in 1957 by Lev Landau as the true symmetry between matter and antimatter [2]. CP violation occurs when there is a difference in the way that particles and anti-particles interact. CP violation was first discovered in 1964, in the decays of neutral kaons by J. Cronin and V. Fitch [3]. Their discovery showed that weak interactions violate not only the charge-conjugation symmetry $\mathrm{C}$ between particles and antiparticles and the parity $\mathrm{P}$, but also their combination. The violation of CP-symmetry plays an important role both in the attempts to explain the dominance of matter over antimatter in the universe, and in the study of weak interactions in particle physics.

The Standard Model Lagrangian contains two ways to break CP-symmetry. The first of these is in the QCD strong sector, which has yet not been found experimentally. The second way, involving the weak force, has been experimentally verified, but only accounts for a small portion of the $\mathrm{CP}$ violation required to explain baryogenesis. Here we present a search for CP violation involving the top quark. 


\subsection{Motivation for searching for $\mathrm{CP}$ violation in single top}

The top quark was discovered in 1995 at the Fermilab Tevatron by the CDF and D0 collaborations [4]. It is the heaviest elementary particle found to date. Its large mass and corresponding coupling strength to the Higgs boson suggests that CP violation in the top sector might be accessible [5].

$\mathrm{CP}$ violation processes can exist within the Standard Model (SM) through a CP-violating phase in the Cabibbo-Kobayashi-Maskawa (CKM) matrix. However, it is estimated that the amount of CP-violation in the SM is not enough to explain baryogenesis, suggesting the possibility that new physics is needed to account for all CP-violating processes [6].

$\mathrm{CP}$ violation is a very rare phenomenon that was confirmed in the decay of $K_{L^{-}}$ mesons [3]. However, the amount of $\mathrm{CP}$ violation observed in these systems can be accommodated within the SM [7]. More interesting are systems involving Bmesons where the expected CP-violating effects within the SM are much larger [8]. On the other hand, the SM predicts very low CP violation for top physics because of the Glashow-Iliopoulos-Maiani (GIM) mechanism which suppresses changes in strangeness by 2 in weak interactions, making any large $\mathrm{CP}$-violation effect direct evidence of physics beyond the SM $[6,8]$. 
The single top process provides a direct measurement of the CKM Matrix element

$\left|V_{t b}\right|$. One of the assumptions in measuring $\left|V_{t b}\right|$ from single top is that the $W t b$ vertex is CP-conserving, and we test that assumption here.

\subsection{Samples selection and single top analysis overview}

This analysis is based on the single top cross section measurement in $s+t, s$ and $t$ channels. (See Section 2.3 for definitions of these channels.) The data and simulation samples went through various selection cuts and processing stages before multivariate and statistical analysis. Sketch of the analysis is shown in Figure 1.1. These items will be discussed in detail in Chapters 4 through 11. 


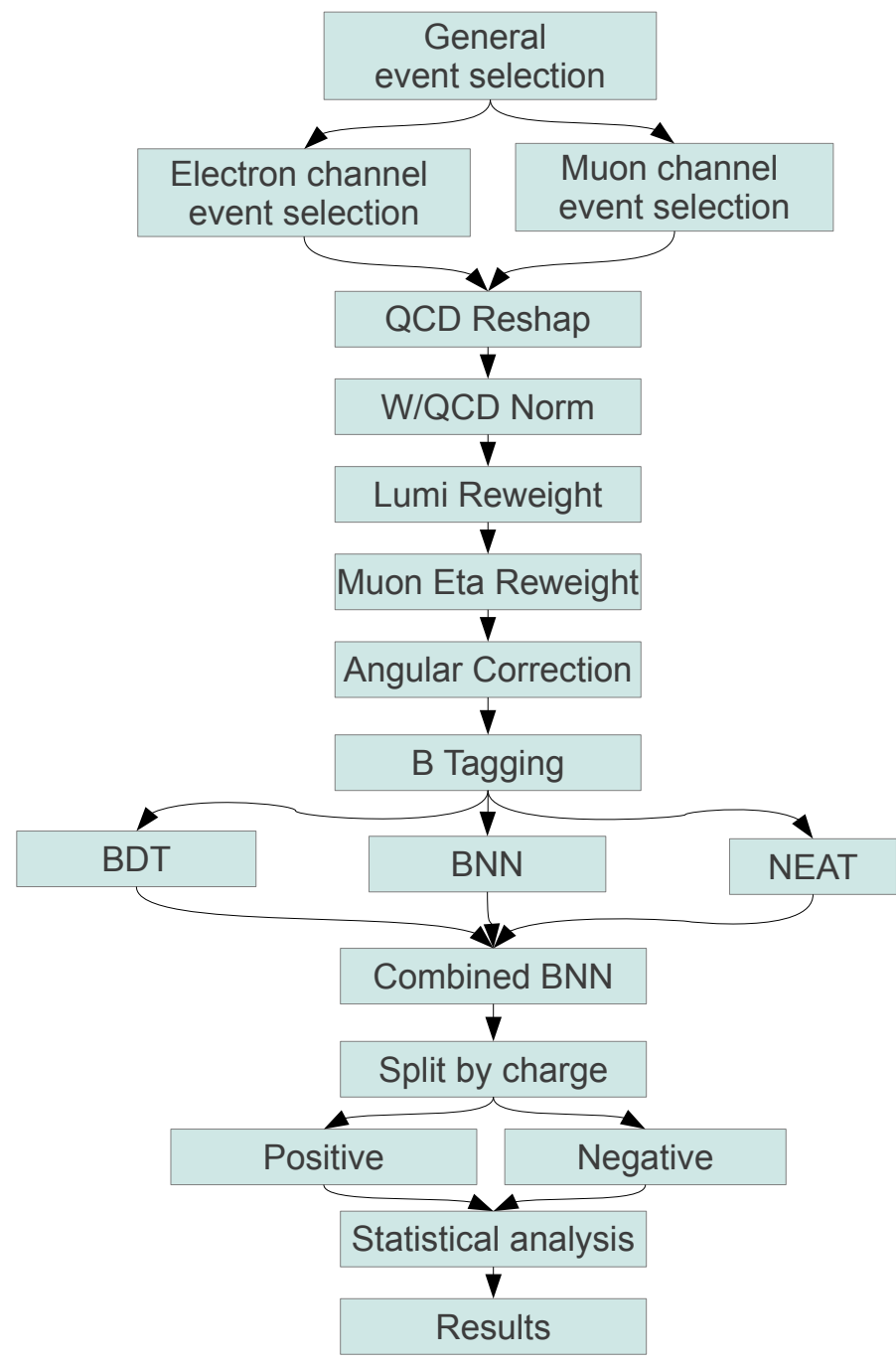

Figure 1.1: Flow chart of the stages of $\mathrm{CP}$ violation analysis in single top. For interpretation of the references to color in this and all other figures, the reader is referred to the electronic version of this dissertation. 


\section{Chapter 2}

\section{Theory}

Elementary particles are the building blocks of the world around us and their interactions have been successfully described by the Standard Model of Elementary Particles $[9,10,11,12]$. High energy experimentalists conduct experiments either to test the SM's predictions in great detail, or to search for new interactions that are not predicted by the SM (Beyound SM). At the energy frontier, high energy accelerators can produce heavy particles such as top quarks to probe new physics.

The top quark is the most massive observed elementary particle. It was postulated in 1973 by Makoto Kobayashi and Toshihide Maskawa to explain CP violation in kaon decays [7]. The top quark was discovered at the Tevatron collider at Fermilab in 1995, with the production of a top-antitop pair via strong interactions [4].

C.-P. Yuan et al [13] and S. Willenbrock [14] predicted a top quark production 
mode by means of the electroweak interaction, in which the top quark is produced singly, thus also called "Single Top" quark production. This production mode has been observed at the Tevatron $[15,16]$ in 2009 by the $D \varnothing$ and CDF collaborations.

Single top is sensitive to many new physics models, and CP violation would be a significant manifestation of them. Moreover, this measurement is unique in the Tevatron ( $p \bar{p}$ collider), and is not easily measured at the Large Hadron Collider (LHC). The last section of this chapter explains how CP Violation in single top manifest itself and how we intend to measure it.

\subsection{The Standard Model}

The Standard Model includes two major theories - quantum electroweak theory and quantum chromodynamics. It describes the interactions between all known particles (electromagnetic, weak, and strong interactions) in terms of one internally consistent framework, the quantum field theory (QFT). The particles in the Standard Model are divided into two groups - fermions and bosons.

The Standard Model has had extensive success in explaining a wide variety of experimental results. Among other examples, the Standard Model predicted the existence of the $\mathrm{W}$ and $\mathrm{Z}$ bosons, gluon, and the top and charm quarks before these particles were observed. Their predicted properties were also experimentally confirmed with good precision. 


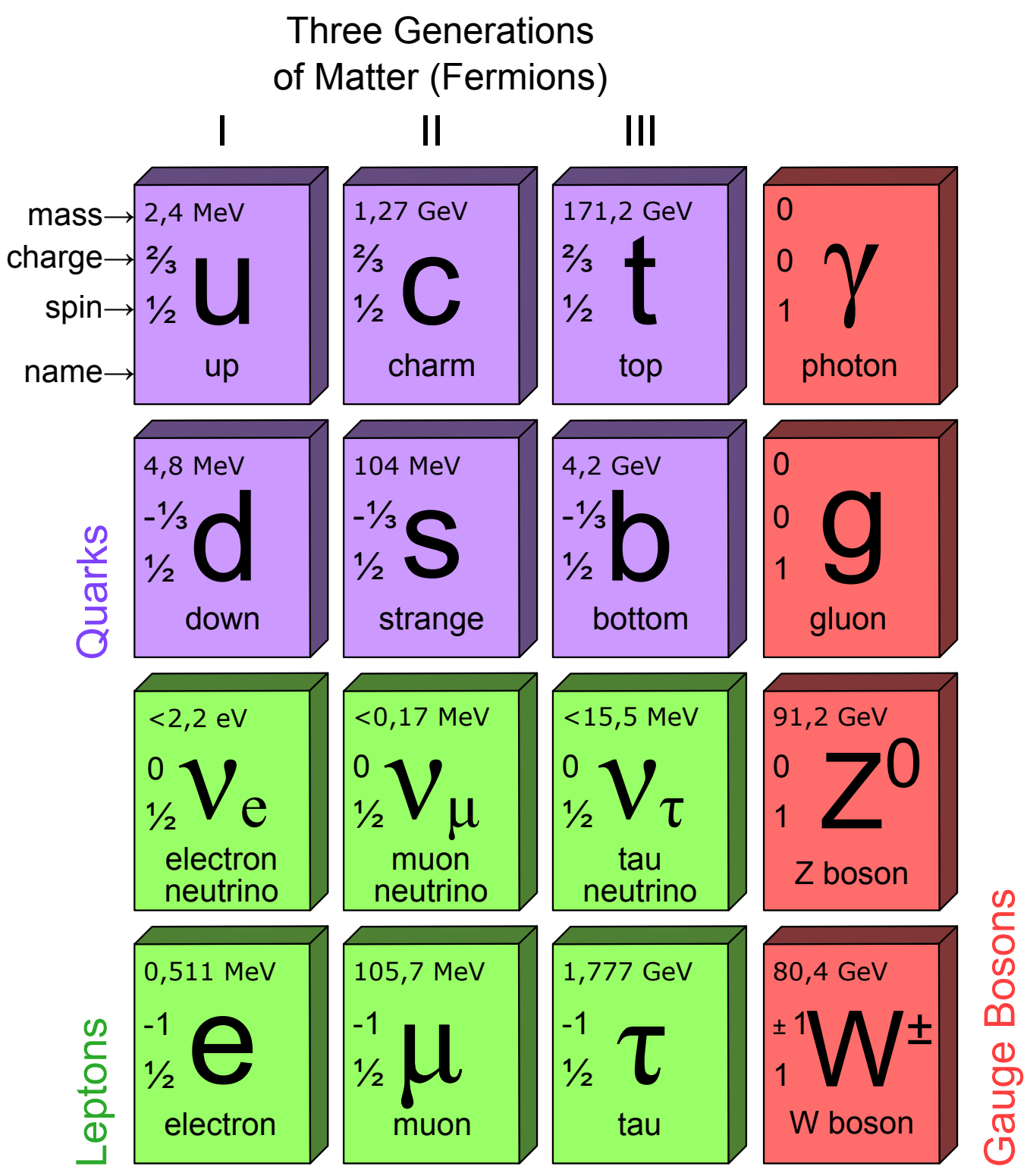

Figure 2.1: The Standard Model of elementary particles. [17] 
The Standard Model depends on 19 parameters, whose numerical values are established by experiment.

\subsubsection{Fermions}

The Standard Model includes 12 elementary particles of spin $\frac{1}{2}$ known as fermions, which observe the Pauli exclusion principle [18]. There are the six quarks (up, down, charm, strange, top, bottom), and six leptons (electron, electron neutrino, muon, muon neutrino, tau, tau neutrino). Each quark/lepton has a corresponding antiparticle with the same mass but opposite electric charge.

The fermions are the building blocks of matter. They are further grouped into three generations, where the lightest are in the first generation and the heaviest in the third. Each generation contains a charged lepton with charge -1 , a charge-neutral neutrino, and up-type and down-type quarks with charges $+\frac{2}{3}$ and $-\frac{1}{3}$ respectively. All ordinary (baryonic) matter is made of first generation charged particles (electrons, and up and down quarks), since these do not decay. On the other hand, the two higher generation charged particles decay within very short half lives.

Some properties of quarks and leptons can be seen in Figure 2.1. Note by massenergy equivalence, we usually express mass in units of $\mathrm{eV} / c^{2}$, where $\mathrm{c}$ is the speed of light in a vacuum, and we set $c^{2}=1$ in this thesis, and then leave out $c$ from equations. The masses of hadrons are mostly of the order of $1 \mathrm{GeV} / c^{2}$, which makes 
the $\mathrm{GeV}$ (gigaelectronvolt, or a billion electronvolt) a very convenient unit of mass. For example the proton has a mass of $0.938 \mathrm{GeV}$.

The quarks carry color charge (red, green, blue), and hence interact through the strong interaction. Quarks also carry electric charge and weak isospin (a component of the weak hypercharge, which unifies weak with electromagnetic interactions.), so they can interact with each other both elctromagnatically and via the weak interaction. At lower energy, or larger distance, the strong interaction becomes very strong, resulting in a phenomena called color confinement, which results in quarks being bound to one another, forming color-neutral composite particles called hadrons. Hadrons are strongly interacting particles built from two types of quark combination: Baryon $=Q Q Q(3$ quarks), Meson $=Q \bar{Q}$ (quark-antiquark pair). The familiar proton $(u u d)$ and the neutron $(u d d)$ are the two baryons composed of quarks with the smallest masses.

The electrons, muons, and taus do not carry color charge, but carry an electric charge, and so they all interact electromagnetically. The neutrinos do not carry color charge or electric charge, so they only interact with the other particles through the weak force, which makes them very difficult to detect. 


\subsubsection{Gauge Bosons}

In the Standard Model, gauge bosons are force carriers that mediate the strong, weak, and electromagnetic fundamental interactions. The gauge bosons of the Standard Model all have spin 1 (thus the name bosons).

Photons mediate the electromagnetic force between particles that carry electric charges. The photon is massless and is described by quantum electrodynamics (QED) [11].

The $W^{+}, W^{-}$, and $Z$ gauge bosons mediate the weak interactions between particles of different flavors (all quarks and leptons). They are massive, with the $Z$ boson the heaviest. These three gauge bosons along with the photons are grouped together, as collectively mediating the electroweak interaction.

The eight gluons mediate the strong interactions between color-charged particles (the quarks). Gluons are massless. The eightfold multiplicity of gluons is labeled by a combination of color and anticolor charge (e.g. red-antigreen). Because the gluon has an effective color charge, they can also interact among with each other, which is different from photons, which do not carry electric charge and therefore do not interact with each other. The gluons and their interactions are described by the theory of quantum chromodynamics (QCD) [11].

Table 2.1 gives a summary of the properties of the gauge bosons in the SM. 
Table 2.1: Standard Model gauge bosons

\begin{tabular}{|c|c|c|c|}
\hline \multicolumn{4}{|c|}{ Gauge Bosons spin $=1$} \\
\hline \hline Force & Gauge boson & Mass $[\mathrm{GeV}]$ & Electric Charge \\
\hline Strong & Gluon $(g)$ & 0 & 0 \\
Weak & $W^{ \pm}$ & $80.398 \pm 0.023$ & \pm 1 \\
Weak & $Z$ & $91.1876 \pm 0.0021$ & 0 \\
EM & Photon $(\gamma)$ & 0 & 0 \\
\hline
\end{tabular}

\subsubsection{Higgs Boson}

The Standard Model also predicts the existence of another boson, the Higgs boson, which gives mass to all elementary particles, including the massive $W / Z$ bosons and the lighter fermions, i.e. quarks and leptons, and even the Higgs boson itself. In this way the Higgs boson is a key building block in the Standard Model.

The Higgs particle was theorized by R. Brout, F. Englert, P. Higgs, G. Guralnik, C. R. Hagen, and T. Kibble in 1964 [19, 20, 21]. It has no intrinsic spin (spin 0), and for that reason is classified as a boson (like the gauge bosons integer spin 1). Because an exceptionally large amount of energy and beam luminosity are theoretically required to observe a Higgs boson in high energy colliders, it is the only fundamental particle predicted by the Standard Model that has yet to be observed. It is hoped that the LHC at CERN will confirm the existence of the Higgs boson. 


\subsubsection{Limits of the Standard Model}

In spite of extensive experimental successes, the Standard Model is not a complete theory of fundamental interactions. It has its own intrinsic defects, and there are numerous phenomena that the SM can not explain. This leads to various Beyond the Standard Model (BSM) searches. Listed below are some of the limitations of the SM:

- It does not incorporate the physics of dark energy, which is the reason that the universe is expanding at an accelerating rate, and accounts for $73 \%$ of the total mass-energy of the universe [22].

- It does not explain gravitation, and it can’t describe general relativity consistently in terms of quantum field theory.

- It does not correctly account for neutrino oscillations and their non-zero masses observed by the oscillation experiments [23]. Although mass terms for the neutrinos can be added to the standard model by hand, the specifics of neutrino mass are still unclear.

- It depends on 19 numerical constants, whose values are obtained from experiment, but the origin of the values is unknown.

- It has several apparently unnatural properties giving rise to puzzles like the strong CP problem, where QCD strong interaction is CP-symmetric, and the 
hierarchy problem, such as why the weak force is $10^{32}$ times stronger than gravity [24].

- It does not contain any dark (i.e. nonluminous) matter particle that possesses all of the required properties deduced from cosmology observations [25].

- It is also difficult to accommodate the predominance of matter over antimatter (matter/antimatter asymmetry).

\subsection{The Top Quark}

The existence of the top quark was postulated (together with bottom quark) in 1973 by Makoto Kobayashi and Toshihide Maskawa to explain the observed CP violations in kaon decay [7]. In hadron colliders, it can be produced via both the strong and electroweak interactions. The top quark was discovered in 1995 by the $D \varnothing$ and CDF Collabrations at Fermilab via strong interactions [4]. The production of single tops via weak interaction was discovered also at Fermilab by the $D \varnothing$ and CDF experiments in $2009[15,16]$.

\subsubsection{Properties}

The top quark is an up-type quark with spin $1 / 2$ and charge $+2 / 3 e$. It has the largest mass of any known elementary particles. Its mass is measured $172.9 \pm 1.5 \mathrm{GeV}[26]$. 
Due to its large mass, it needs high energy colliders to be created, such as Tevatron or LHC. It participates in strong, weak and electromagnetic processes.

\subsubsection{Production}

In hadron collisions, top quarks are produced dominantly in pairs through the QCD

processes $q \bar{q} \rightarrow t \bar{t}$ and $g g \rightarrow t \bar{t}$. Approximately $85 \%$ of the production cross section at the Tevatron is from $q \bar{q}$ annihilation, with the remainder from gluon-gluon fusion. Smaller cross sections are expected from electroweak single-top productions mechanisms, mediated by virtual $s$-channel and $t$-channel $W$ bosons, respectively. A virtual particle is one having mass not equal to that of the free particles, also called "off mass shell".

A Feynman diagram for top pair production is shown in Fig. 2.2

\subsubsection{Decay}

Top quarks decay almost exclusively into a $W$ boson and a $b$ quark, with a branching ratio $B(t \rightarrow W+b) \approx 99.8 \%$. Thus the final decay products are determined by the W boson decay.

The fraction of top events containing $b$ quarks is expected to be close to $100 \%$, and the quarks to be rather energetic, since they come from the decay of a massive object. 


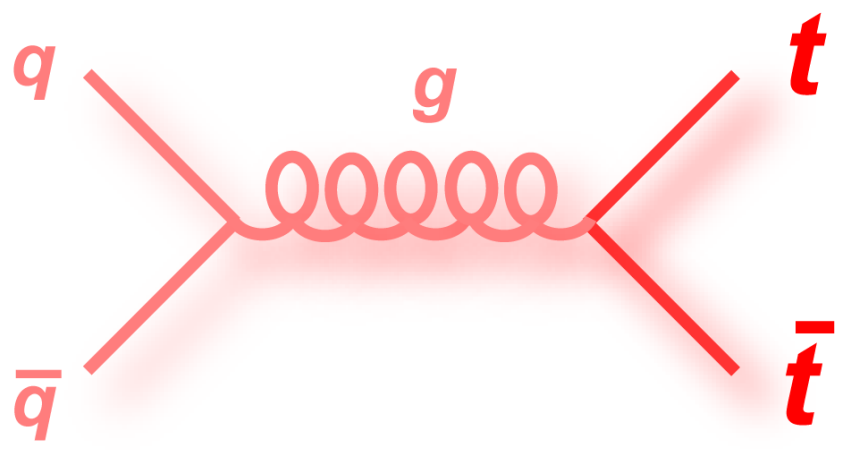

Figure 2.2: Leading order Feynman diagram for the production of top quark pairs in strong forces, where a quark-antiquark pair form a very energetic gluon, which then decays into a top-antitop pair. From here and below, for the Feynman diagrams, the time axis is always from left to right, and the space axis is from bottom up, unless otherwise noted.

Because of its enormous mass, the top quark has an extremely short lifetime of

only $5 \times 10^{-25} \mathrm{~s}$, which is about 20 times smaller than the time scale of the strong interaction $\left(10^{-23} \mathrm{~s}\right)$. As a result, unlike other quarks, the top quark is expected to decay before top-flavored hadrons or tt-quarkonium bound states can form. This provides a unique opportunity to study directly the decay of quarks.

\subsection{The single top quark}

\subsubsection{Single top production and decay at the Tevatron}

Electroweak top quark production is usually referred to as single top quark production because only one top quark is produced per event. At hadron colliders, 
there are three Standard Model single top quark production modes, the $s$-channel $(p \bar{p} \rightarrow t \bar{b}+X) 2.3$ and $t$-channel $(p \bar{p} \rightarrow t q \bar{b}+X) 2.4$ exchanges of a virtual $W$, and the $t W$ production $(p \bar{p} \rightarrow t W+X)$. The next to leading order (NLO) cross sections with NNLL corrections for these processes at the Tevatron at $1.96 \mathrm{TeV}$ are listed in Table 2.2. The central values of the cross sections for single top are for estimated 172.5 GeV top quark mass. The uncertainties include components from the choice of scale and parton distribution functions, but not for the top quark mass.

Table 2.2: Single top quark production cross sections [1].

\begin{tabular}{|c|c|}
\hline Process & Cross Section $(p b)$ \\
\hline$t$-channel & $2.26 \pm 0.12$ \\
$s$-channel & $1.04 \pm 0.04$ \\
\hline
\end{tabular}

At the Tevatron, the two dominant single top quark production channels are the $s$-channel and $t$-channel. In the $s$-channel, an intermediate virtual time-like $W$-boson decays into a top and antibottom quark, as shown in Figure 2.3. The final state is therefore a $W$ boson and two bottom jets. In the $t$-channel, a bottom quark (originating from gluon radiation) transforms to top quark by exchanging a virtual space-like $W$ boson with an up or down quark, as shown in Figure 2.4. The accompanying bottom quark is typically at large pseudorapidity (See definition in Equation 3.6) and low transverse momentum, defined as $p_{T}=\sqrt{p_{x}^{2}+p_{y}^{2}}$. Thus they usually es- 
cape detection, and are often not reconstructed in the analysis. The $t$-channel final state results also dominantly in $W+2$ jets, with just one of the jets coming from a bottom quark. Occasionally, the initial or final-state quark can radiate a hard gluon or quark at a large angle. That can be detected as additional jets. Together with the light quark, this will lead to $W+3$ and $W+4$ jets events. The number of jets reconstructed in the detectors depends on the decay kinematics. Although as shown in Figure 2.4 is a $2 \rightarrow 3$ process, it contributes similar large cross section in the NLO calculation to $s$-chanel [27]. The $t W$ production mode, where a single top quark is created in association with a real (on-shell) $W$ boson, has too small of a cross section $(0.14 \pm 0.03 \mathrm{pb}[1])$ at the Tevatron energy, so it's not included in our search channels.

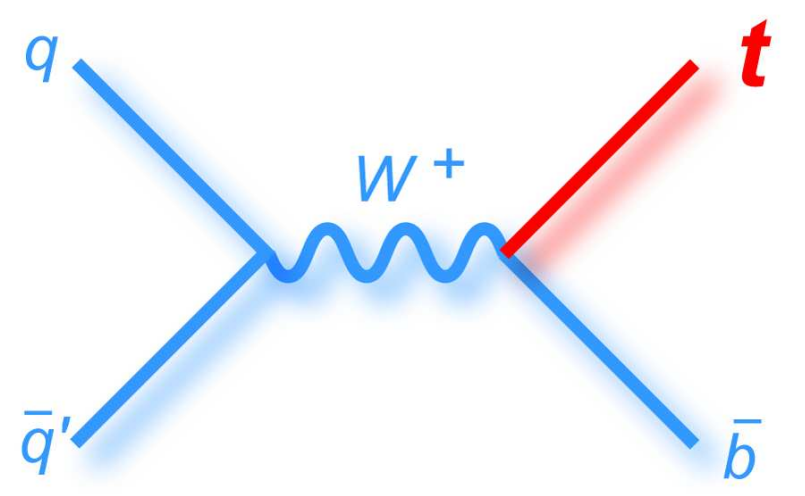

Figure 2.3: Feynman diagram for single top s-channel production.

As mentioned in the top quark section 2.2, the top quark decays to a $W$ boson 


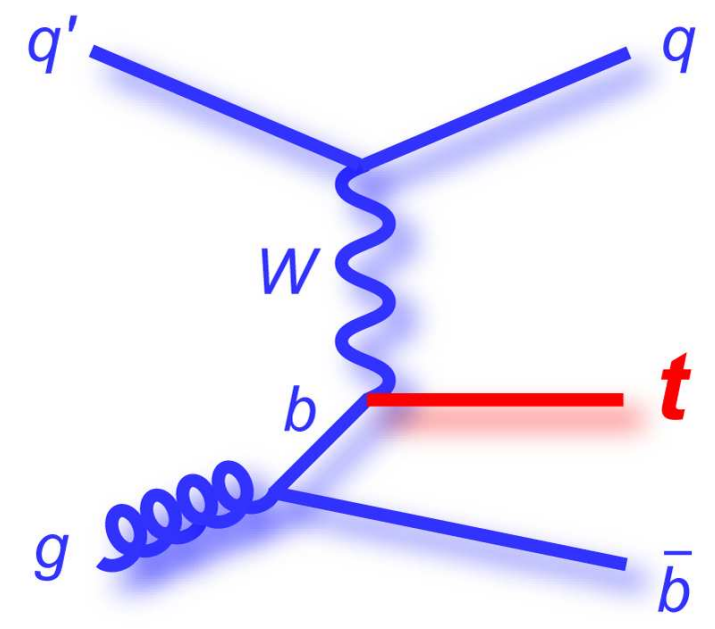

Figure 2.4: Feynman diagram for single top $t$-channel production.

and a $b$ quark. The $W$ boson further decays to two jets or to a lepton plus neutrino (Figure 2.5). The all-jets channel is immersed in a large QCD background. In our analysis, the electron and muon leptonic final states are the most useful. The Feynman diagrams for the single top production and decay modes for $s$ - and $t$ channel are shown in Figure 2.6 and Figure 2.7 separately.

Due to its large mass, the top quark is produced almost at rest, and the $b$ quark from the top quark decay tends to be central and have large transverse momentum, as shown in Figure ??. For the decay products of $W$ boson, the lepton has a softer $p_{T}$ spectrum than that of the neutrino. This is due to the $W$ boson polarization, so the lepton from the $W$ boson decay preferentially points in the same direction as the incident proton or antiproton. The lepton appears as an isolated track of 


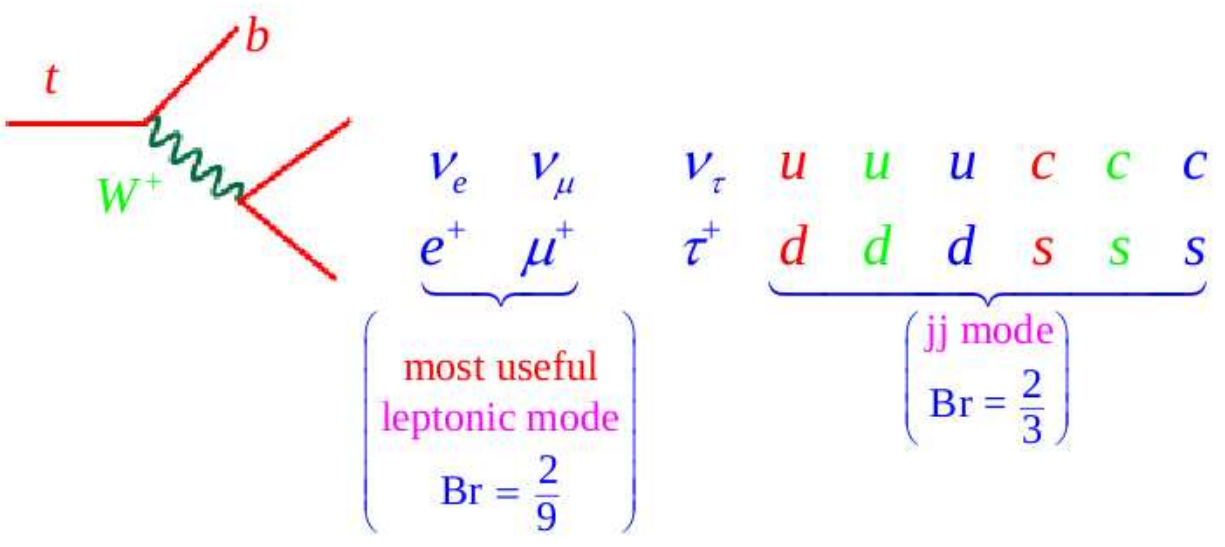

Figure 2.5: Decay branching ratio of a top quark. Source: CP Yuan class notes.

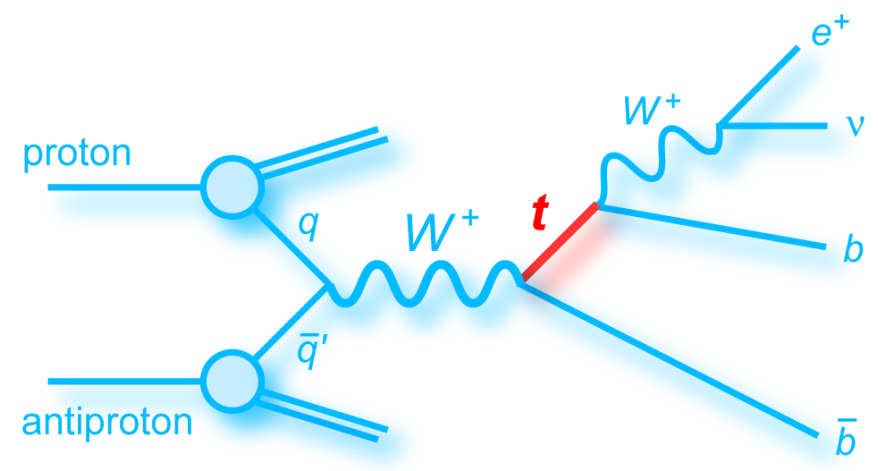

Figure 2.6: Feynman diagram for single top $s$-channel production and decay. 


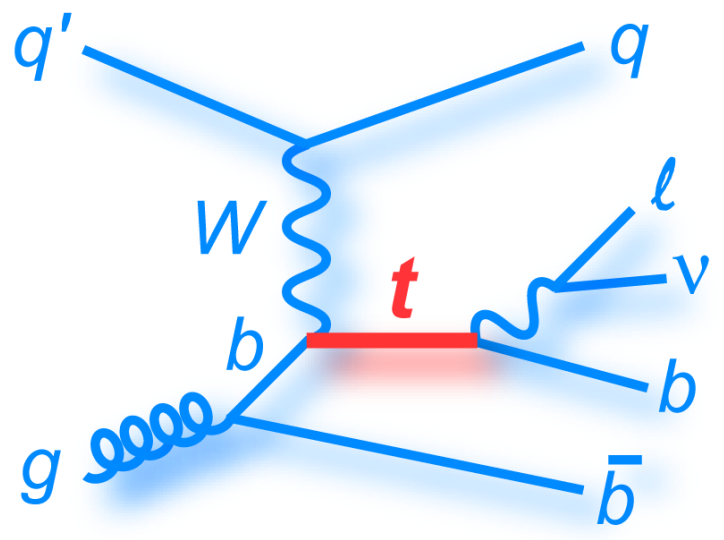

Figure 2.7: Feynman diagram for single top $t$-channel production and decay.

high momentum in the cetnral tracking detector, matched with a shower in the electromagnetic segments of the calorimeter or the muon chamber. The transverse momenta of neutrinos from the $W$ boson decay are reconstructed from the imbalance in transverse energy measured in each event (missing ET):

$$
E_{T}=-\sum E_{T}^{i}
$$

(Other quarks involved in the collision generate secondary hadrons, but they are mostly low $\left.p_{T} \cdot\right)$

Several single top parton level kinematics distributions for both channels are shown in Figure 2.8 to Figure 2.11 .

The light quark produced in the $t$-channel has reasonably large $p_{T}$, but its most distinguishing feature is the asymmetric $Q(l) \times \eta$ distribution, where $Q(l)$ is the 


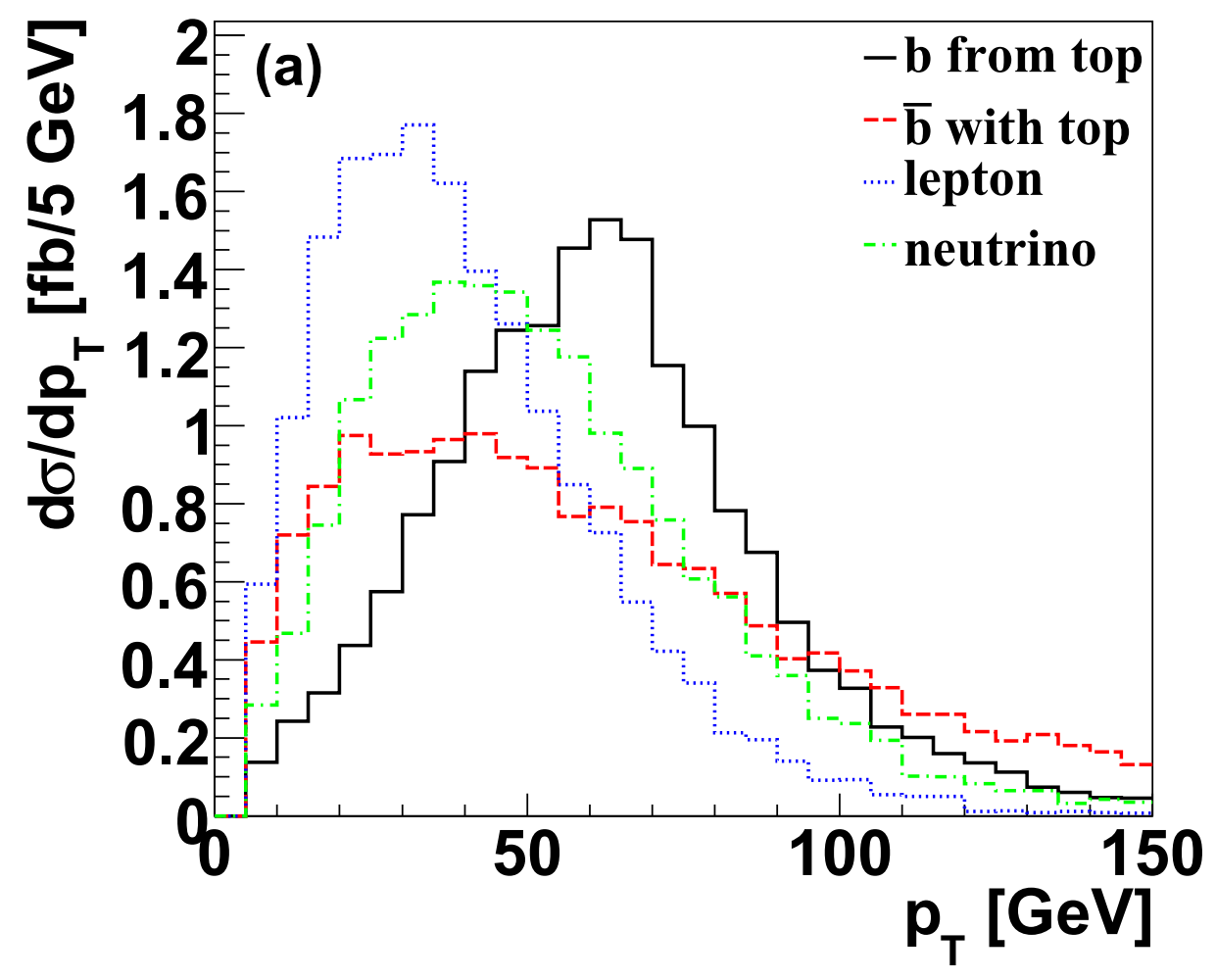

Figure 2.8: The transverse momentum for $s$-channel 


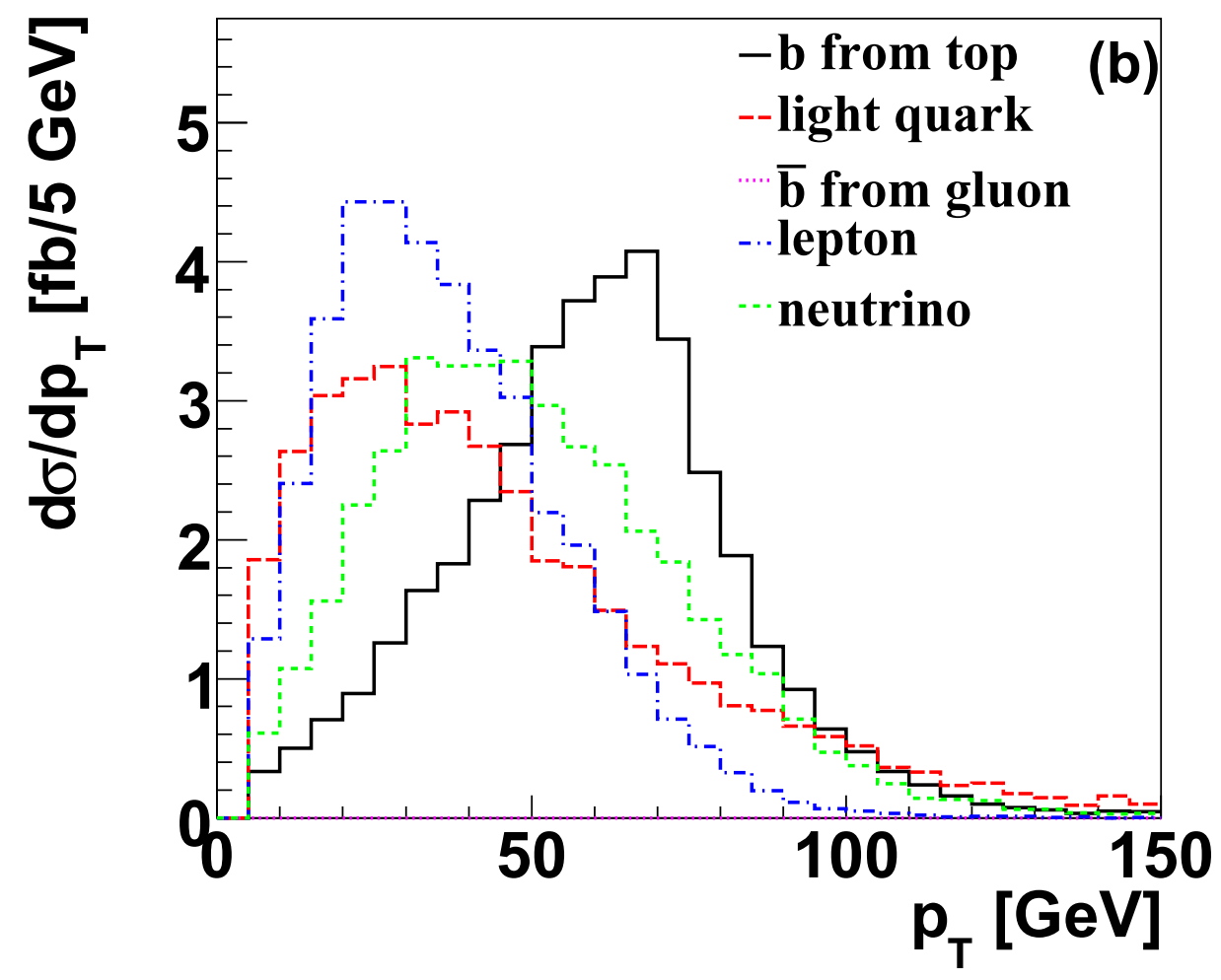

Figure 2.9: The transverse momentum for $t$-channel 


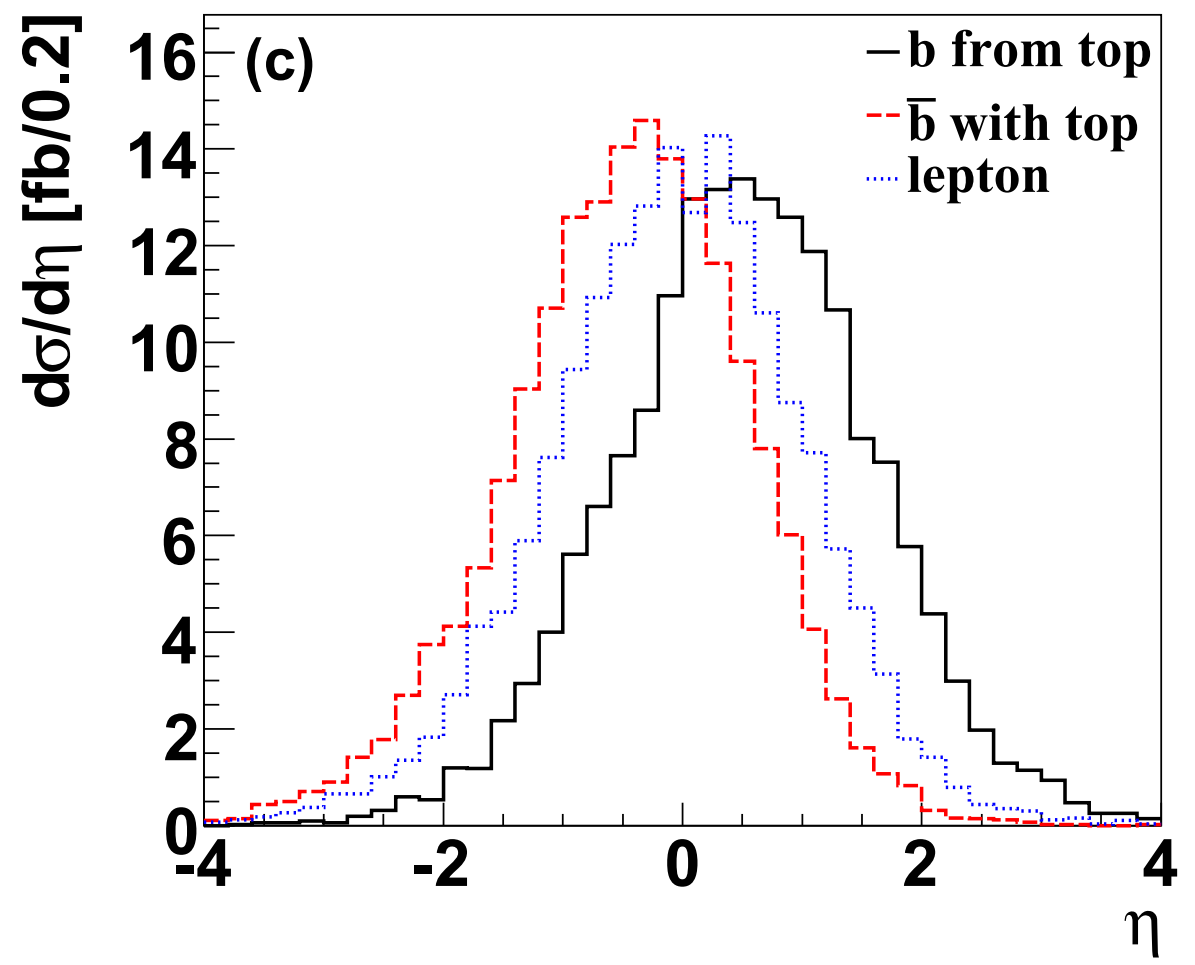

Figure 2.10: The pseudorapidity for $s$-channel 


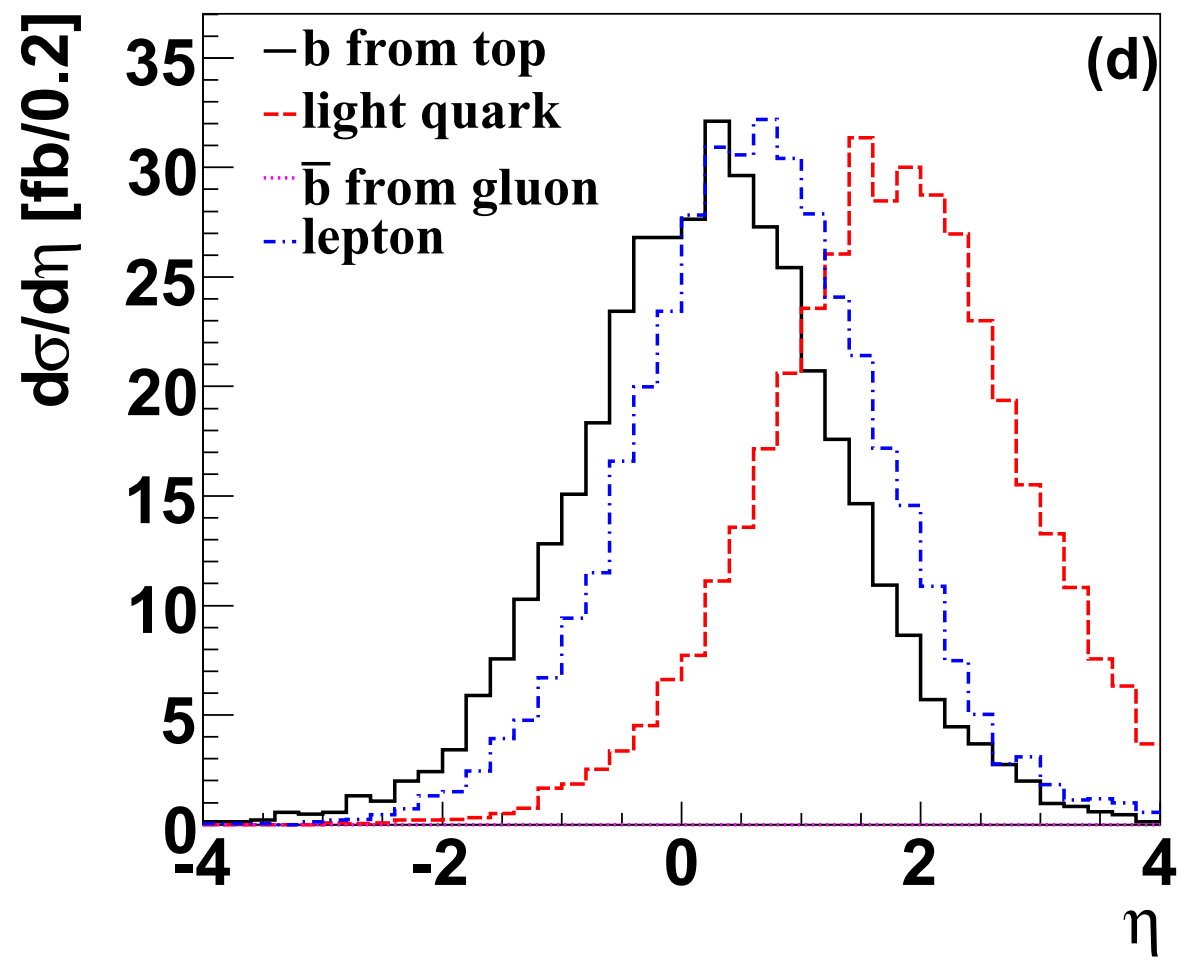

Figure 2.11: The pseudorapidity for $t$-channel 
charge of the lepton in the event, which is used to keep the multiplication positive. This asymmetry arises because the final state light quark produced during the single top production most often is a $d$ quark that moves in the same direction as the proton (antiproton). The light quark $\eta$ will hence tend to have the same sign as the charge of the lepton from the top decay.

Event selection therefore requires a high $p_{T}$ electron or muon, two to four jets, one of which must be identified as originating from a bottom quark, and missing ET (Details of event selection see Chapter 5).

\subsubsection{Motivations for searching for CP Violation in single top}

The main significance of measuring the cross sections for single-top production is that

it is directly proportional to the $\left|V_{t b}\right|^{2}$ component of the CKM matrix. Moreover, no assumption is needed on the number of families or on the unitarity of the CKM matrix to measure $\left|V_{t b}\right|$. If there is $\mathrm{CP}$ violation, it would show up for amplitude in $\left|V_{t b}\right|$

Single top quark production offers excellent opportunities to probe physics beyond the standard model. There are several ways new physics could produce a single top quark differently for top and anti-top through processes such as new exchange particles, flavor changing neutral currents (FCNC), anomalous $W t b$ couplings or 
other processes.

Single top can be produced by the decay of a resonance, for example, a $W^{\prime}$ boson, which have the same final states as the SM single top production, as shown in Figure 2.12. If this resonance couples differently to top and anti-top, this could produce different cross sections.

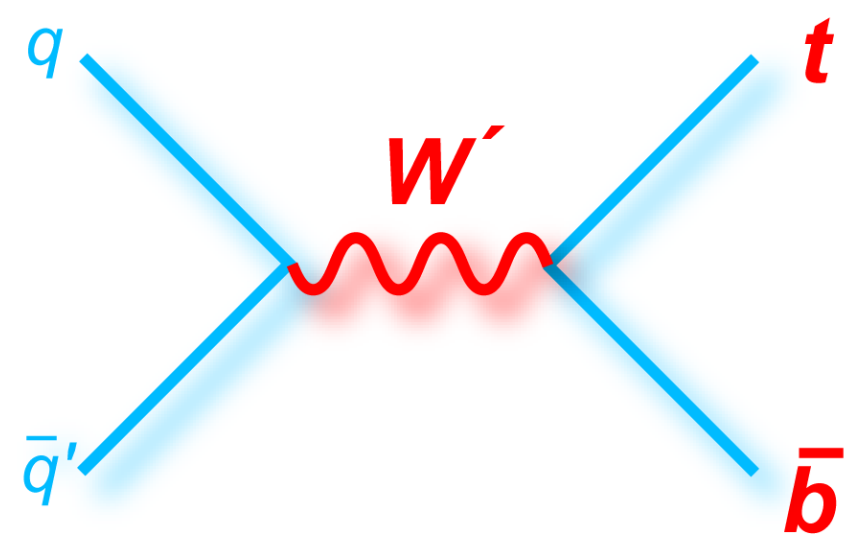

Figure 2.12: Feynman diagram for $W^{\prime}$ boson decaying into single top.

Non-SM interactions, such as flavor changing neutral currents (FCNC), can also enhance the total single top cross section for $t$-channel, and possibly introduce a difference in top and anti-top production rates. The Feynman diagrams for FCNC via exchange of a gluon are shown in Figure 2.13.

In summary, the $s$-channel $(t b)$ single top production is most sensitive to new, heavy charged bosons. The $t$-channel $(t q b)$ single top quark production would similarly be enhanced by flavor-changing neutral currents (FCNC). Either could produce 


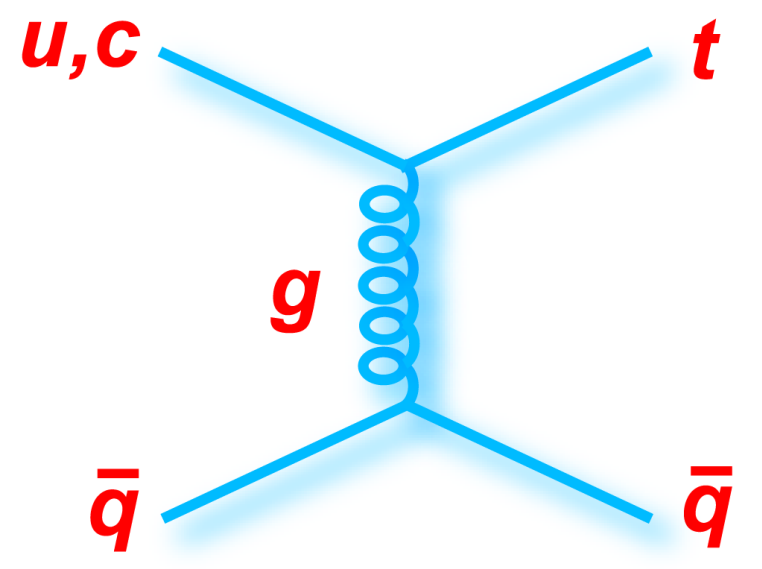

Figure 2.13: FCNC via exchange of a gluon in single top production.

a CP asymmetry effect, providing sensitivity to new physics.

\subsection{Outline of CP violation measurement in single top}

Combining the CP symmetry with simultaneous time reversal (T) produces a combined symmetry called CPT symmetry. As of now, no violations of CPT symmetry have been observed. CPT symmetry must be preserved in any Lorentz invariant local quantum field theory with a Hermitian Hamiltonian.

We exploit that Tevatron is a $p \bar{p}$ collider, meaning that the initial state is a $\mathrm{CP}$ eigenstate and therefore, any difference between the cross sections for $p \bar{p} \rightarrow t X$ and $p \bar{p} \rightarrow \bar{t} X$ would be a clear indication of $\mathrm{CP}$ violation $[8,28]$. 
The purpose of this measurement is to measure the top and anti-top quark production separately. The theoretical cross section for each of them is half that of the total single top cross section.

The asymmetry $\mathcal{A}_{P}$ in the single top production can be expressed as follows:

$$
\mathcal{A}_{P}=\frac{\sigma(p \bar{p} \rightarrow t X)-\sigma(p \bar{p} \rightarrow \bar{t} X)}{\sigma(p \bar{p} \rightarrow t X)+\sigma(p \bar{p} \rightarrow \bar{t} X)}
$$

where $\sigma$ denotes the production rate.

The strategy of this analysis is to identify the production of single $t$ or $\bar{t}$ quarks using the charge of the lepton when the $W$ boson decays leptonically and then measure the individual single $t$ and $\bar{t}$ production cross sections. We assume that no significant $\mathrm{CP}$ violation is present in background samples.

This analysis uses the same event selection and signal and background modeling as the measurement of the single top quark production cross section using $5.4 \mathrm{fb}^{-1}$ of DØ Run IIa and Run IIb data [29]. We split by lepton charge the discriminant that was used to measure the single top quark production cross section [30], and use it to measure top and antitop production cross sections separately. Therefore all the systematic uncertainties in the single top quark cross section measurement are applied to this analysis $[29,30]$. A new systematic uncertainty is added to account for the misidentification of the lepton charge, discussed in detail in Appendix D. 


\section{Chapter 3}

\section{Experimental Setup}

The Fermi National Accelerator Laboratory (Fermilab) [31, 32] is located in the west surburbs of Chicago, Illinois in the USA. Fermilab is the only facility in the world where high energy protons and anti-protons collide at a center-of-mass energy of $\sqrt{s}=1.96 \mathrm{TeV}[32,33]$. The Tevatron completed its physics program in September 2011 and stopped running. All the other elements in the accelerator chain except the Tevatron and recycler are still being used for neutrino physics and other experiments.

A complex detection system is required to "see" the details of these energetic collisions, and study interesting physics processes such as single top quark production. The two main detectors at Fermilab are known as D $\varnothing$ and CDF. This chapter presents a description of accelerator complex and the DØ detector at Fermilab. 


\subsection{The Accelerator Complex and Tevatron}

Fermilab uses a series of accelerators to create the high energy proton and antiproton beams that collide in the Tevatron collider. The proton beams are created starting with hydrogen gas from a linac at the beginning of the accelerator cycle. A portion of the proton beams are taken from the Main Injector to create antiprotons. Once enough antiprotons are accumulated, they are loaded into the Tevatron, where they are accelerated to the final highest energy stage. The accelerated protons and antiprotons are grouped into a number of bunches, and they collide at two points where the $\mathrm{CDF}(\mathrm{B} 0)$ and $D \varnothing(\mathrm{D} 0)$ detectors are located. Figure 3.1 is a picture illustrating Fermilab's accelerator chain. Below we talk about each part separately.

\section{- Cockcroft-Walton Pre-accelerator}

Producing negatively charged hydrogen ions $\left(H^{-}\right)$is the first step in creating proton and antiproton beams. This takes place in the Cockcroft-Walton generator. Hydrogen gas is converted into $\mathrm{H}^{-}$ions by introducing it into a

container lined with molybdenum electrodes. The ions are accelerated out of the container by a $750 \mathrm{keV}$ electrostatic field applied by the Cockcroft-Walton generator.

\section{- Linear Accelerator}

The Fermilab Linac is a 500-foot long linear particle accelerator that accelerates 


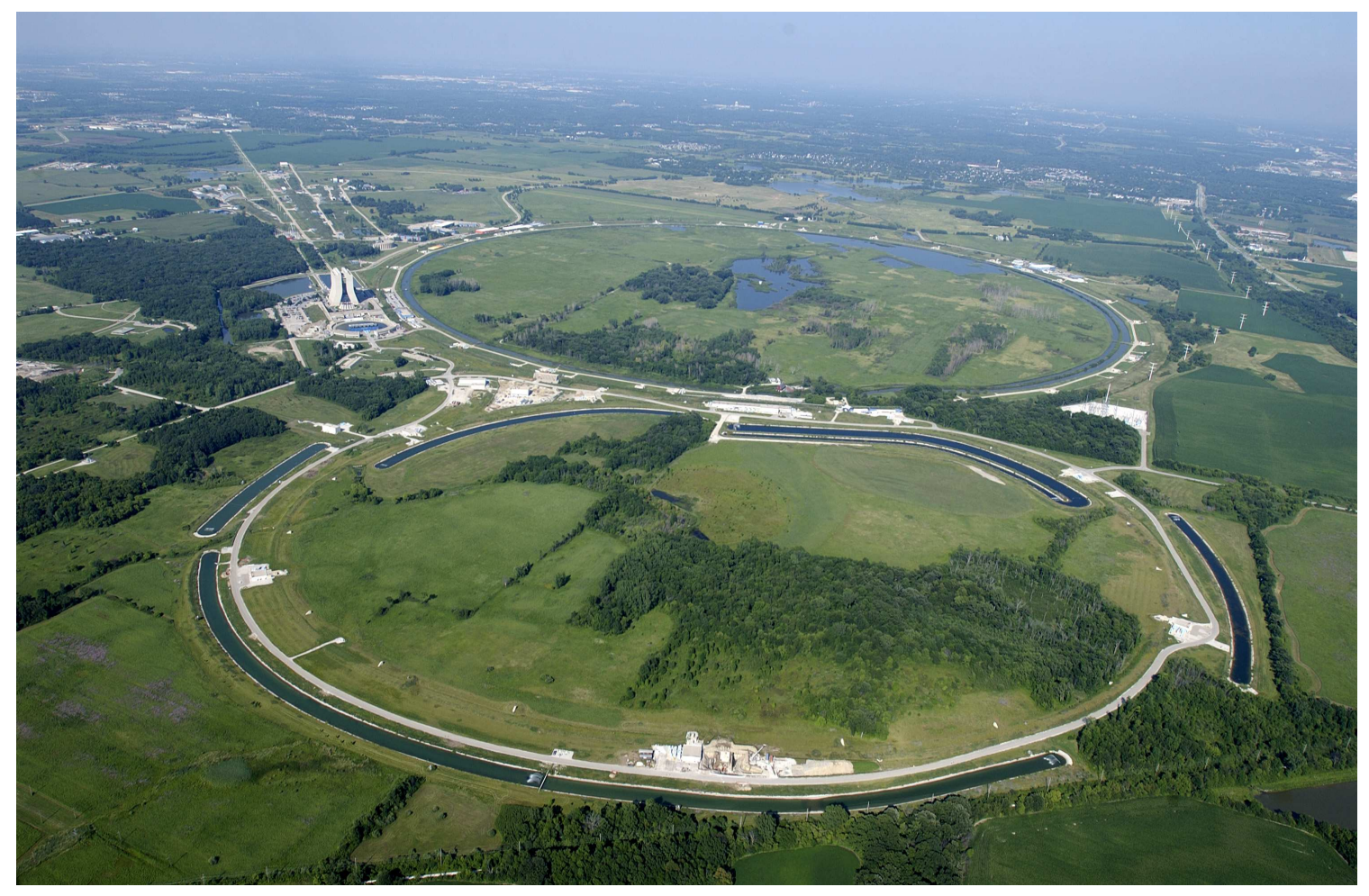

Figure 3.1: Fermilab's accelerater chain. 
the negative hydrogen ions $H^{-}$from the Cockcroft-Walton pre-accelerator to $400 \mathrm{MeV}$ before injecting them into the Booster accelerator. The $\mathrm{H}^{-}$ions pass through a carbon foil, which removes electrons from the hydrogen ions, creating positively charged protons or $H^{+}$.

\section{- Booster}

Located nearly 20 feet below ground, the Booster is a circular accelerator with a diameter of 500 feet that uses magnets to bend electrically charged particles in a circular path. Boosting means the protons coming from the linac experience a repeated acceleration from electric fields during each revolution, increasing their energy by a small amount each time. The protons travel around the Booster about 20,000 times, and their energy is boosted to $8 \mathrm{GeV}$ by the end of the acceleration cycle. Then the Booster sends the protons to the Main Injector.

In the synchrotron, the final beam energy is determined by the ring radius and the maximum value of field:

$$
p=0.3 B \rho,
$$

where $p$ is the proton momentum, B is the magnetic field in Tesla, and $\rho$ is the ring radius in meters.

- Main Injector The Main Injector (MI) is the next link in the accelerator chain. 
The MI performs three functions: it accelerates protons and antiprotons for injection into the Tevatron, it delivers protons for antiproton production, and it transfer antiprotons between antiproton storage rings and from the antiproton storage rings to the Tevatron. Protons from the Main Injector are also delivered to several neutrino targets.

\section{- Antiproton Source}

Antiprotons do not exist freely in nature like normal matter. To produce antiprotons, a $120 \mathrm{GeV}$ beam of protons taken from the main injector is steered onto the side of a drum shaped nickel target. The drum is rotated quickly to prevent the beam from hitting the same spot and thus preventing the destruction of the target. The collisions produce many secondary particles including antiprotons. The aniprotons then enter a beamline in which they are captured and focused, before being injected into a storage ring. After accumulating a sufficient number of antiprotons in the storage ring, they are sent to the Recycler before they are injected into the Tevatron.

\section{- The Recycler}

The Fermilab Recycler is a $8 \mathrm{GeV}$ permanent magnet storage ring for the accumulation of antiprotons from the antiproton source, and the recovery of the antiprotons remaining at the end of a Tevatron store. It is located in the 
Main Injector tunnel directly above the Main Injector beamline.

\section{- The Tevatron}

The Tevatron is the most powerful proton-antiproton accelerator in the world. The Tevatron ceased operations on September 30th, 2011. It accelerates beams of protons and antiprotons to nearly the speed of light at an energy of 0.98 $\mathrm{TeV}$ around a four-mile circumference vacuum pipe mostly surrounded by superconducting electromagnets. 774 niobium-titanium superconducting dipole magnets cooled in liquid helium are used to produce a magnetic filed of 4.2 Tes-

las. Another $240 \mathrm{NbTi}$ quadrupole magnets are used to focus the beam [31]. The collisions reproduce conditions in the early universe $(\approx 10$ ps after the Big Bang, in comparison to 1 ps for LHC) and probe the structure of matter at a very small distance. Picture 3.2 shows the Tevatron tunnel.

\subsection{Luminosity and Cross Sections}

In particle physics, collision rates are measured in terms of instantaneous luminosity, L. The luminosity is an important value to characterize the performance of an accelerator. Luminosity is defined as the number of particles per unit area per unit time, usually expressed in cgs units, $\mathrm{cm}^{-2} \mathrm{~s}^{-1}$.

For an intersecting storage ring collider, the instantaneous luminosity can be 


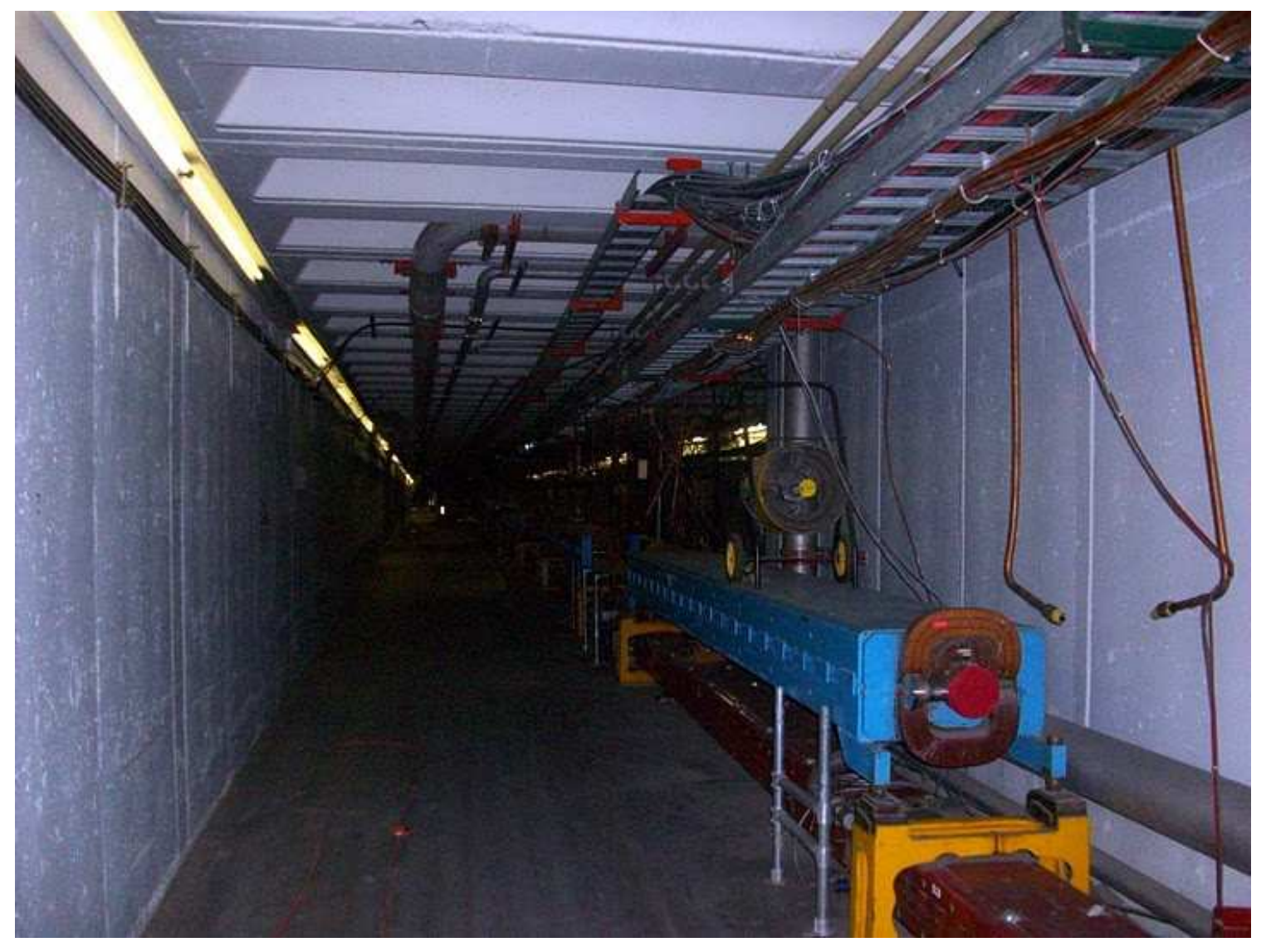

Figure 3.2: Fermilab's Tevatron Tunnel. The upper magnets are not in use. The lower magnets are part of the Tevatron Collider. Protons and antiprotons travel through a vacuum pipe located inside the string of magnets. On the roof are the cables and cooling pipes. 
characterized by the parameters:

$$
L=f n \frac{N_{1} N_{2}}{A}
$$

where

$f$ is the revolution frequency,

$n$ is the number of bunches in one beam in the storage ring,

$N_{i}$ is the number of particles in each bunch,

and $A$ is the cross area of the beam.

The higher the luminosity, the greater the chance that a proton will collide with an antiproton. The Tevatron had been able to deliver luminosities up to $4 \times 10^{32}$ $\mathrm{cm}^{-2} \mathrm{~s}^{-1}[34]$. At the interaction point, the beams are squeezed very narrowly to increase the chance of hard collisions.

The rate of a physics process is given by

$$
\frac{d N}{d t}=L \sigma
$$

where $\sigma$ is the total cross section of the process, $\mathrm{N}$ is the count of physics events, and $\mathrm{L}$ is the (instantaneous) luminosity.

The total number of collisions which result in a given physics process in a period 
of time can thus be written as

$$
N=\sigma \int L d t=\sigma \mathcal{L}
$$

where $\mathcal{L} \equiv \int L d t$, the integrated luminosity, is defined as the integral of the instantaneous luminosity with respect to time.

Therefore, the collision count can be calculated by multiplying the integrated luminosity by the cross-section for that collision process. The total number of collisions is directly proportional to the integrated luminosity over this time.

The integrated luminosity has the unit of inverse area, usually expressed as inverse picobarns $p b^{-1}$ or inverse femtobarns $f b^{-1}\left(1\right.$ barn $=10^{-24} \mathrm{~cm}^{2} ; 1 \mathrm{fb}=10^{-15}$ barns $\left.=10^{-39} \mathrm{~cm}^{2}\right)$.

This analysis uses $5.4 \mathrm{fb}^{-1}$ of data collected at $D \varnothing$.

\subsection{The $D \varnothing$ detector}

Detectors are our "eyes" to record and identify the useful events to reveal the nature of fundamental interactions. $D \varnothing$ is one of two detectors used to study collisions produced in the Tevatron. Proton-antiproton collisions create showers of new particles at the center of both $\mathrm{CDF}$ and $D \varnothing$ detectors at a rate of more than 2 million $\mathrm{Hz}$. We cannot record all those events. The trigger system decides whether an event 
is interesting enough to be worth keeping. About 20 events per second are stored on computer tape. For these interesting events, the detectors record each particle's flight path, energy, momentum and electric charge.

$D \varnothing$ is a typical high energy particle detector. It measures thirty feet tall and fifty feet long and consists of four major parts: a tracking system, calorimeter, muon system, trigger system. Figure 3.3 gives a sketch of the $D \varnothing$ detector components.

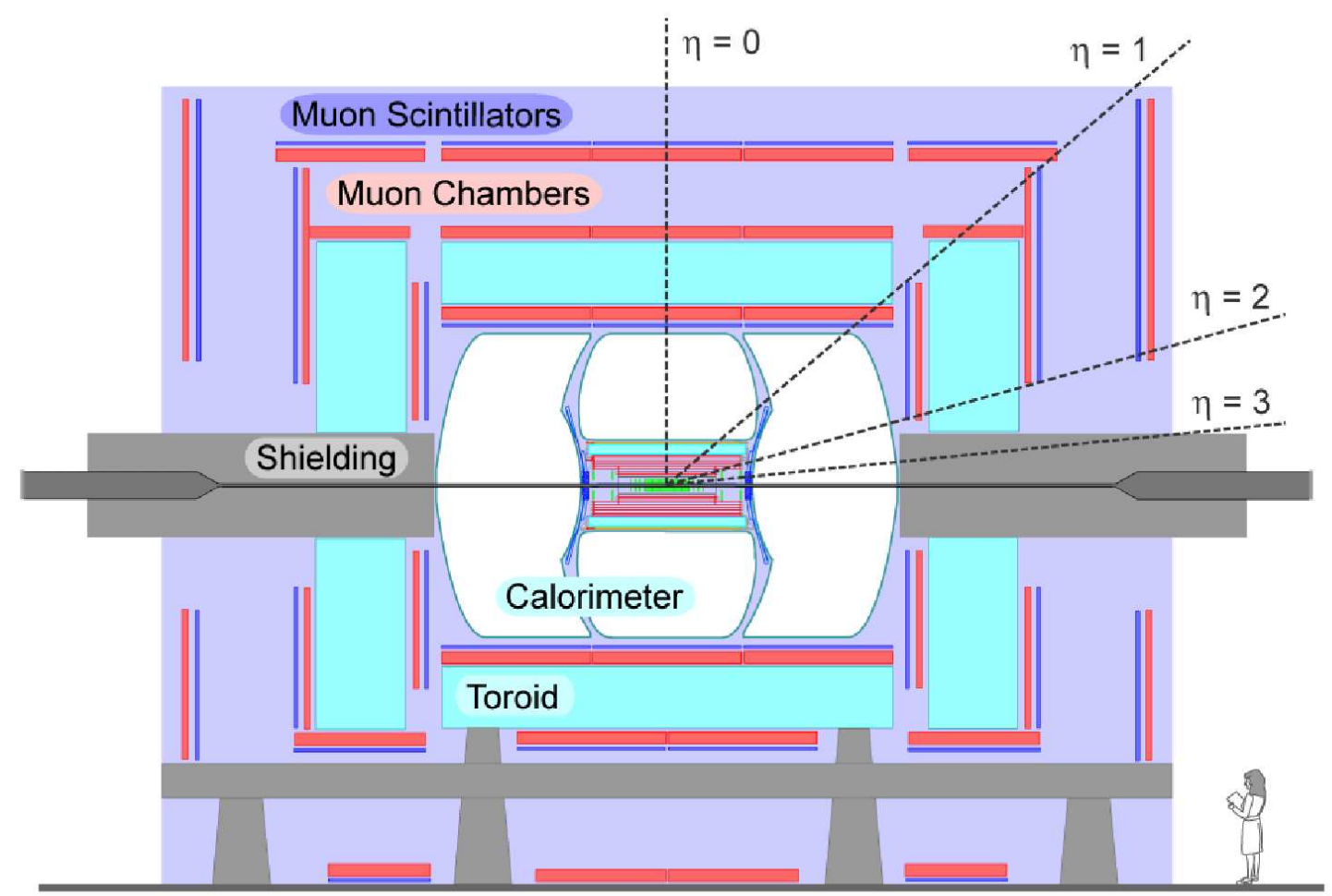

Figure 3.3: Side view of the $D \varnothing$ detector. The proton/anti-proton beam travels from left/right to right/left. 


\subsubsection{Particle Signatures}

Stable particles such as $p, \bar{p}, e^{ \pm}, \gamma$ will show up in the detector as energy deposits in hadronic and electromagnetic calorimeters or charged tracks in the tracking system. Muons have a long lifetime and interact less with matters in the detector, which means they pass through the entire detector before they decay, and leave tracks in the muon detectors. Most of the particles produced in high-energy collisions are short-lived. In order to observe all possible decay products of an collision, one needs a hermetic detector and electronic system to record the events for further offline analysis. Besides, since the presence of a neutrino is inferred from the missing energy, it's important that the calorimeters have $4 \pi$ solid angle coverage, so that missing energy is not due to any failure to detect all produced particles. Figure 3.4 shows a typical cylindrical modern detector:

Figure 3.5 shows what signatures particles will leave in the components of the detector.

- Most charged particles, like electrons and protons, are detected both in the tracking chamber and the electromagnetic calorimeter.

- Muons have a long life time, and have small interactions with matters in calorimeter, so they are detected in all parts of the detector. They pass through the entire detector, and leave tracks in the muon chamber. 


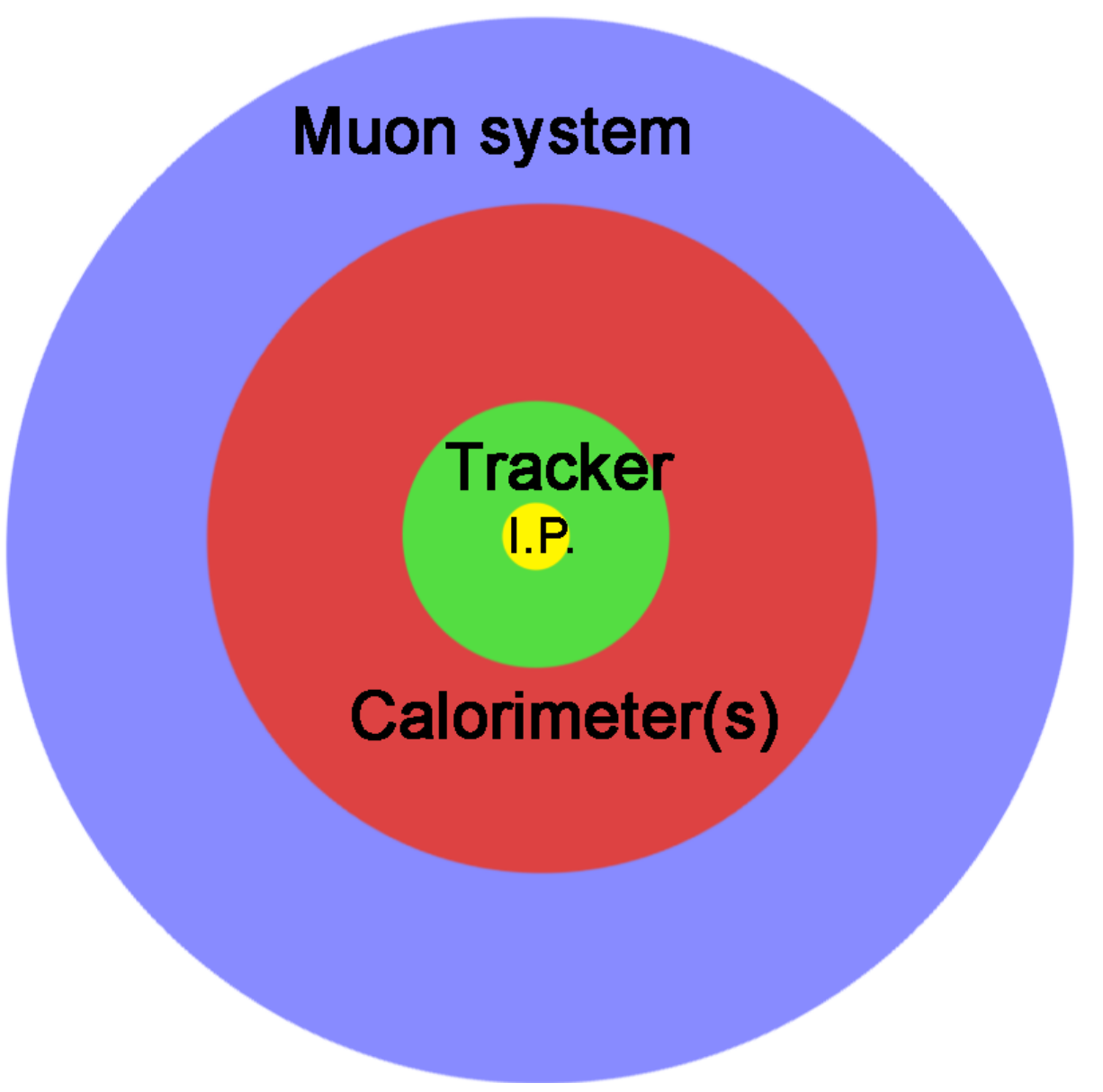

Figure 3.4: A schematic of the basic components of a hermetic detector. I.P. refers to the region containing the interaction point. This is a cross section of the typical cylindrical design. [35]. 


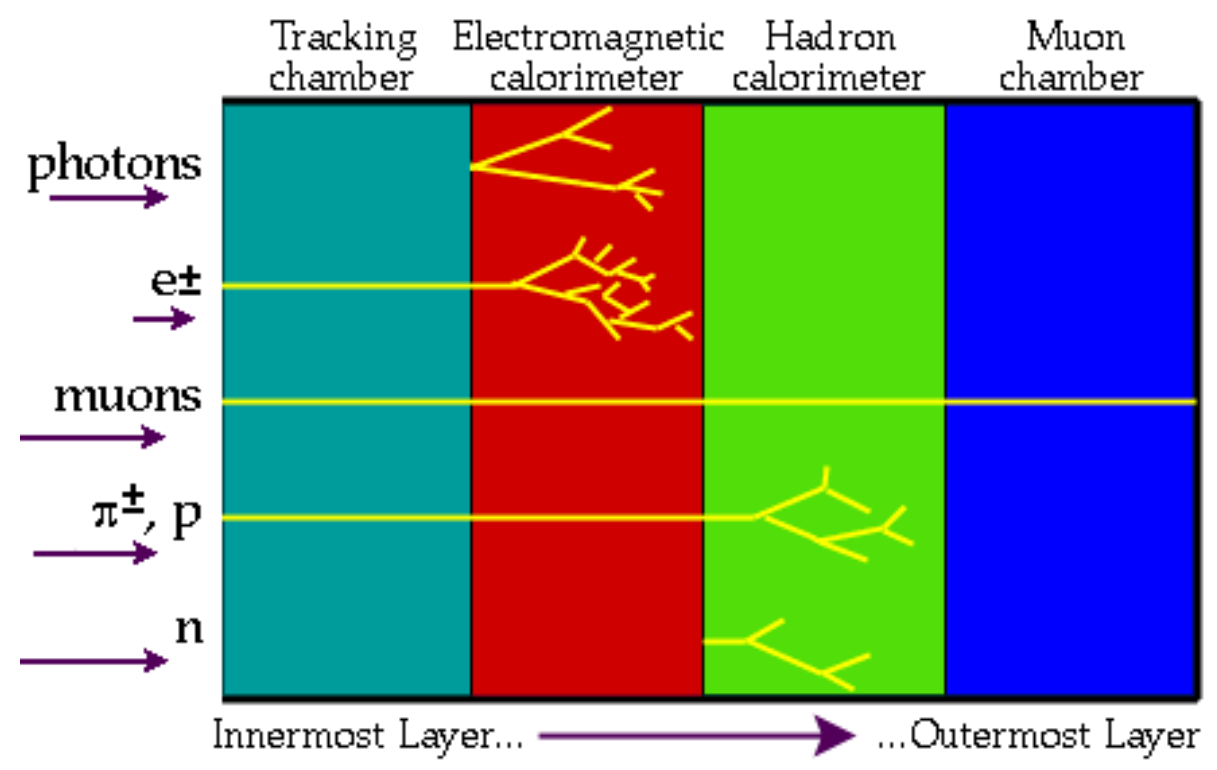

Figure 3.5: High energy particle signatures left in the detector components [36].

- Neutral particles, like neutrons and photons, are not detectable in the tracking chamber.

- Photons are detected by the electromagnetic calorimeter, while neutrons are evidenced by the energy they deposit in the hadron calorimeter.

- Neutrinos rarely interact with matter, and will escape from detection by the detector. Their presence can only be inferred using missing energy by looking at the momentum balance (Eq. 2.1).

An event consists of all data pertaining to a single $p \bar{p}$ interaction as measured in the detector. 


\subsubsection{Coordinate System and Pseudorapidity}

For the convenience of the $D \emptyset$ detector description and data analysis, we use a righthanded coordinate system, in which the z-axis is defined along the beam pipe in the direction of the proton beam, and the $\mathrm{y}$-axis is upward, and thus $\mathrm{x}$-axis directed into the paper by right-handed rule (Figure3.3). The (x, y)-plane is usually referred to as the transverse plane, which is vertical to the beam direction (z-axis). The polar angle $\theta$ is the angle relative to the positive z-axis, and the azimuthal angle $\phi$ is the angle from the positive $\mathrm{x}$-axis on the transverse plane.

The rapidity of a particle of momentum $p^{\mu}$ is defined as

$$
y=\frac{1}{2} \ln \left(\frac{|\mathbf{p}|+p_{z}}{|\mathbf{p}|-p_{z}}\right)
$$

where $p_{z}$ is the component of the momentum along the beam axis.

In the Tevatron, the particles are traveling close to the speed of light, thus the mass of the particle is neglectable, and $E \approx|\mathbf{p}|$ establishes. In this limit, the rapidity becomes equal to the pseudorapidity:

$$
y=\frac{1}{2} \ln \left(\frac{E+p_{z}}{E-p_{z}}\right)=-\frac{1}{2} \ln \left[\tan \left(\frac{1-\cos \theta}{1+\cos \theta}\right)\right]=-\ln \left[\tan \left(\frac{\theta}{2}\right)\right] \equiv \eta,
$$

where $\eta \equiv-\ln \left[\tan \left(\frac{\theta}{2}\right)\right]$ is called the pseudorapidity, and $\theta$ is the angle between the particle momentum $\mathbf{p}$ and the beam axis. 
We use the term "forward" to describe the regions that are close to the beam axis at large $|\eta|$ (e.g. 2.5), and "central" means the regions with small $|\eta|$ (e.g. 1.5). Pseudorapidity depends only on the polar angle of the trajectory of a particle, and not on its energy as seen from Eq. 3.6.

The detector pseudorapidity $\eta^{\text {det }}$ is defined as the pseudorapidity of the calorimeter cluster (electron, jet, or central track muon) with respect to the geometric center of the $D \varnothing$ detector, $\eta^{\text {det }} \equiv-\ln \left[\tan \left(\frac{\theta_{\text {det }}}{2}\right)\right]$, where $\theta_{\text {det }}$ is relative to the center of the detector.

\subsubsection{The Central Tracking System}

The point where the beams collide is surrounded by tracking detectors to record the tracks (trajectories) of high energy particles produced in the collision. The Tracking System is closest to the interaction point. It includes a silicon micro-strip tracker (SMT) and a central fiber tracker (CFT) right outside the silicon detector. The whole tracker is immersed in a $2 \mathrm{~T}$ superconducting solenoidal magnet, thus particle tracks are curved; from the curvature we can deduce their momentum. The SMT and CFT are optimized for tracking and vertexing at pseudorapidities $|\eta|<3$ and $|\eta|<2.5$, respectively. A schematic view of the central tracking system is shown in Figure 3.6.

The Tracking System is used to identify and measure the momentum of electrons 


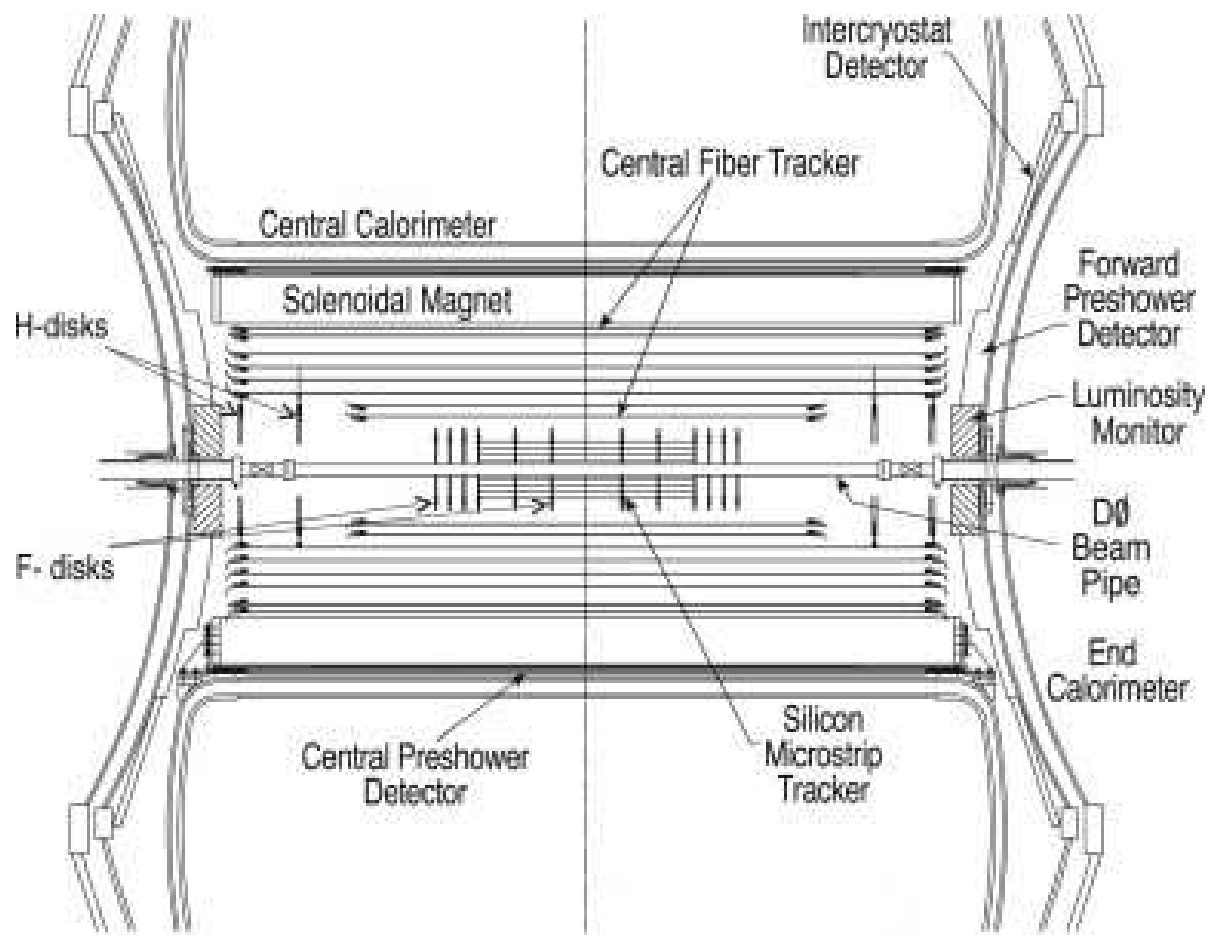

Figure 3.6: The Tracking System at $D \varnothing$. 
and muons, and to determine the position of the primary interaction vertex. The two tracking detectors are able to locate the primary interaction vertex with a resolution of about $35 \mu \mathrm{m}$ along the beamline. They are also used to identify jets originating from $b$ quarks, which is an important decay product of single top.

\subsubsection{Silicon Micro-strip Tracker (SMT)}

The silicon micro-strip tracker (SMT) is located closest around the interaction region. It gives very precise information, but silicon is expensive, so it is installed closest to the beam where they cover a smaller area. The SMT is used for both tracking and vertexing.

The SMT is designed of barrel modules interspersed with disks in the center, and disks in the forward region (Figure 3.7). The barrel detectors primarily measure the $r-\phi$ coordinate and the disk detectors measure $r-z$ and $r-\phi$. Vertices of particles in the forward region are reconstructed in three dimensions by the disks, and vertices of particles in the central region are measured in the barrels, and the central fiber tracker. The detector has in total six barrels and sixteen disks.

\subsubsection{Central Fiber Tracker (CFT)}

Outside the silicon, $D \varnothing$ has an outer tracker made of scintillating fibers, which produce photons of light when a particle passes through. The CFT consists of scintillating fibers mounted on eight concentric support cylinders and occupies $r$ from 


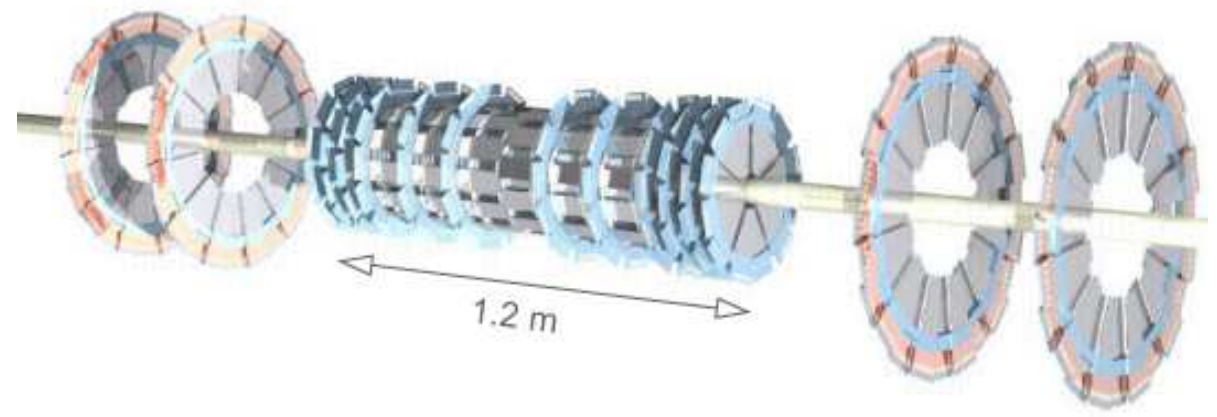

Figure 3.7: The disk/barrel design of the silicon microstrip tracker.

20 to $52 \mathrm{~cm}$ from the center of the beampipe. The fibers are double clad and are 835 microns in diameter. The two innermost cylinders are $1.66 \mathrm{~m}$ long; the outer six cylinders are $2.52 \mathrm{~m}$ long. The outer cylinder provides coverage for $|\eta|<1.7$ (See Fig. 3.6).

Each cylinder supports two layers of fiber doublets. One doublet layer of fibers is oriented along the beam direction $(\mathrm{z})$ and a second doublet layer is oriented at a stereo angle in $+3^{\circ}$ or $-3^{\circ}$.

The scintillating fibers are coupled to clear fiber waveguides which carry the scintillation light to visible light photon counters for read out. The entire detector has 71,680 channels.

\subsubsection{The Central and Forward Preshower Detectors}

The central and forward preshower detectors are located between the solenoid and the calorimeters. The preshower detectors aid in electron identification and back- 
ground rejection during both triggering and offline reconstruction. They function as calorimeters as well as tracking detectors, enhancing the spatial matching between tracks and calorimeter showers.

The central preshower detector (CPS) covers the region $|\eta|<1.3$. The CPS consists of three concentric cylindrical layers, each having 1280 triangular scintillator strips. The resolution of the CPS is about $6 \mathrm{~mm}$. The two forward preshower detectors (FPS) cover $1.5<|\eta|<2.5$.

\subsubsection{The Calorimeters and ICD}

The $D \varnothing$ calorimeter system consists of three uranium/liquid-argon calorimeters and an intercryostat detector (ICD).

\subsubsection{Calorimeters}

The calorimeters are located outside the tracking system. They use uranium metal bathed in liquefied argon to capture particles and measure their energies. The calorimeters provide energy measurements for electrons, photons, and jets, as well as assist in identification of electrons, photons, jets, and muons and measure the transverse energy imbalance in events (missing transverse energy $\mathbb{E}_{T}$ ). They play crucial role in this analysis. The calorimeter systems are illustrated in Figure 3.8 below. 


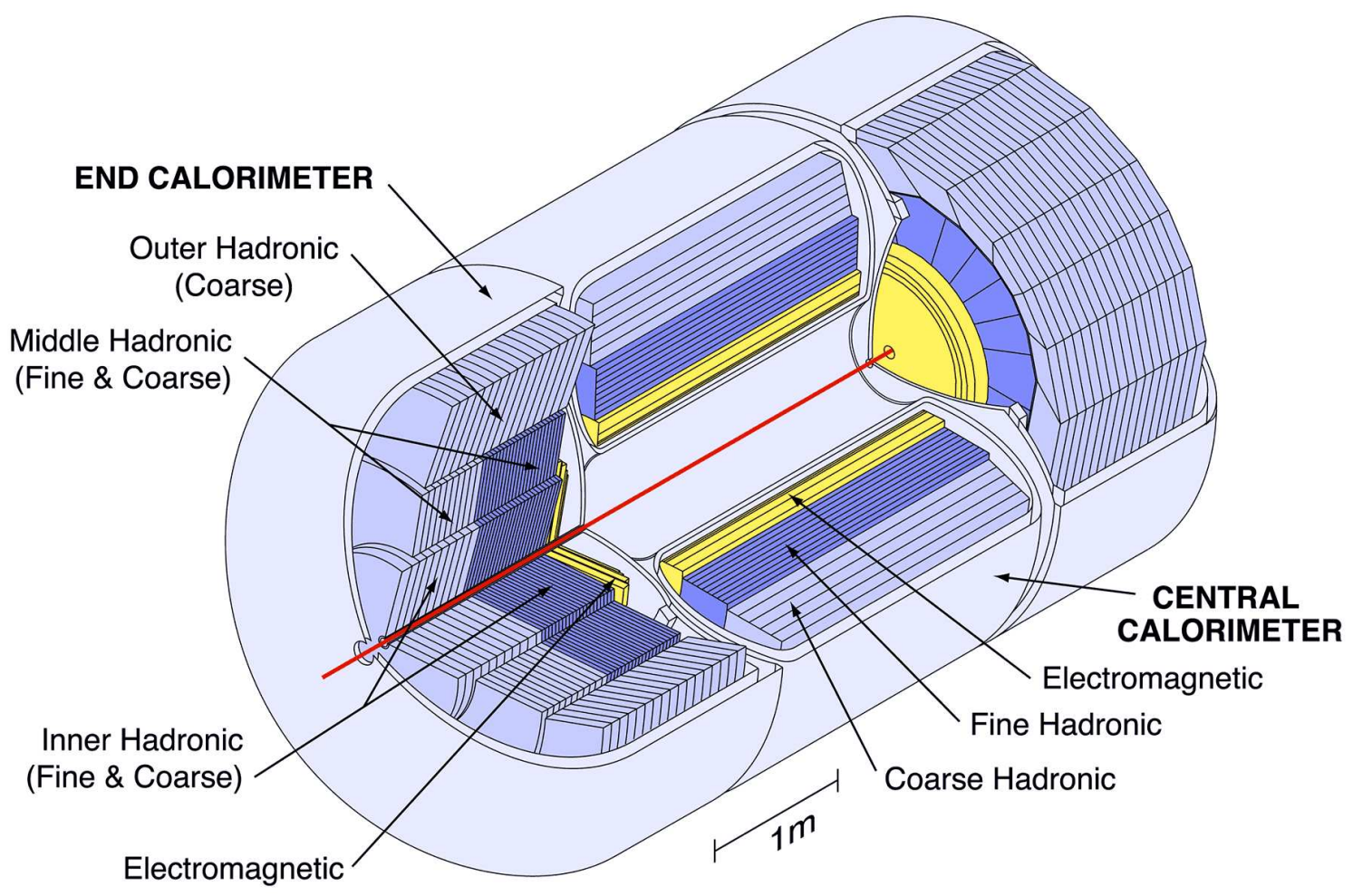

Figure 3.8: Illustration of the calorimeter systems. 
As can be seen in Figure 3.8, each calorimeter contains an electromagnetic section closest to the interaction region followed by fine and coarse hadronic sections.

As shown in Figure 3.9, the calorimeter has a central section (CC) covering pseudorapidities up to $|\eta| \approx 1.1$, and two end calorimeters (EC) that extend coverage to very forward region $|\eta| \approx 4.2$. All three calorimeters are housed in separate cryostats that maintain a temperature of approximately $90 \mathrm{~K}$.

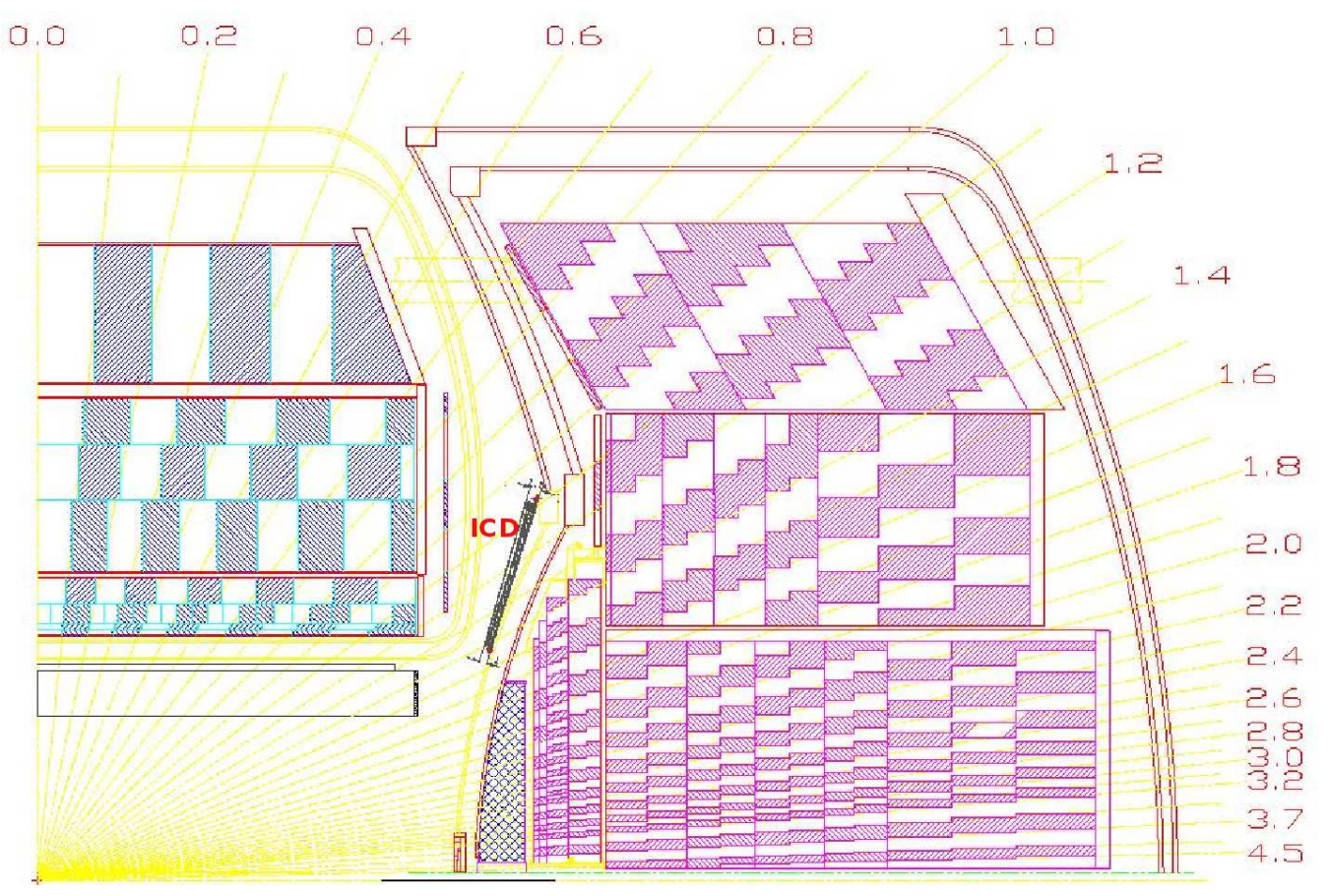

Figure 3.9: Intersecting view of a quarter of the $D \varnothing$ calorimeter, showing the trigger towers and pseudorapidity coverage. The shading pattern indicates groups of cells ganged together for signal readout. The rays indicate pseudorapidity intervals from the center of the detector. The ICD is also denoted in the figure.

The signals generated by particle interactions in the calorimeters are grouped into showers. Calorimeter readout cells form pseudo-projective towers as shown 
in Figure 3.9, which are called Trigger Towers, which are essential for L1 and L2 triggers. There are 55,296 calorimeter electronic channels to be read out in total.

\subsubsection{Intercryostat Detector}

There are two issues with the calorimeters:

- The three calorimeters provide incomplete coverage in pseudorapidity.

- There is substantial material in the region we do not analyze, thus degrading the energy resolution.

To address these problems, additional single-cell structure layers of sampling have been added between the central and end cryostats. This Intercryostat detector (ICD) consists of scintillator sampling that is attached to the exterior surfaces of the end cryostats. It covers the region $1.1<|\eta|<1$.4. Its rough location is shown in Figure 3.9.

\subsubsection{The Muon System}

Surrounding all of the rest of the detector, the muon system resides at the most outside layer beyond the calorimeter, and as the name implies, detects muons. Unlike most common particles, muons don't get absorbed in the calorimeter; they interact small with the calorimeter and live long enough to leave the detector. By putting 
detectors outside, we can identify muons relatively easily. High energy muons are quite rare and a sign of interesting collisions.

The central muon system uses proportional drift tubes (PDTs), central scintillation counters. The PDTs and scintillators are emerged in toroidal magnets in order to bend charged particles. The scintillators are also used for triggering. The central muon system provides coverage for $|\eta|<1.0$.

The forward muon system extends muon detection to $|\eta| \approx 2.0$, and uses mini drift tubes (MDTs) rather than PDTs, and includes trigger scintillation counters and beam pipe shielding.

\subsubsection{The Luminosity Monitor}

Luminosity at the $D \varnothing$ interaction region is measured by plastic scintillator arrays placed in front of the EC cryostats. A dedicated Luminosity Monitor (LM) is used to detect inelastic pp collisions. The LM also serves to measure beam halo rates and as a fast measurement of the $\mathrm{z}$ coordinate of the interaction vertex.

The LM detector consists of two arrays of twenty-four plastic scintillation counters with Photomultiplier tubes (PMTs) readout. The arrays are located in front of the end calorimeters and occupy the radial region between the beam pipe and the forward preshower detector. They cover the pseudorapidity range $2.7<|\eta|<4.4$. 


\subsubsection{The $D \varnothing$ Trigger System}

Proton-antiproton collisions delivered by the Tevatron happen inside the detector 2.5 million times every second. We cannot record all those events on computer tape. Instead, trigger and data acquisition systems are designed to accommodate the high interaction rates. The trigger is a system of fast electronics and computers that decides whether an event is interesting enough to be kept using information from a small subset of the $D \varnothing$ detector signals. The task of the trigger system is to suppress background events as efficiently as possible while not losing precious physics events.

Three distinct levels form this trigger system with each succeeding level examining fewer events but in greater detail.

- The first stage (Level 1 or L1) comprises a collection of hardware trigger elements that provide a trigger accept rate of about $2 \mathrm{kHz}$.

- In the second stage (Level 2 or L2), hardware engines and embedded microprocessors associated with specific subdetectors provide information to a global processor (L2GBL) to construct a trigger decision based on individual objects as well as object correlations. Once there is a L2 accept, the complete detector information is read out. The L2 system has an accept rate of approximately 1 $\mathrm{kHz}$.

- Events passed by L1 and L2 are sent to a farm of Level 3 (L3) microprocessors; 
sophisticated algorithms reduce the rate to about $50 \mathrm{~Hz}$ to be recorded for offline reconstruction.

The overall coordination and control of $D \emptyset$ triggering is handled by the software package COOR running on the online host. COOR interacts directly with the trigger framework (for L1 and L2 triggers) and with the data acquisition supervising systems (for the L3 triggers).

\subsubsection{The Level 1 Trigger}

The Level 1 Trigger is implemented in hardware and programmable firmware. The calorimeter trigger (L1Cal) inputs consist of electromagnetic and hadronic trigger tower energies made up of sums over depth and transverse coordinates (in a $\Delta \eta \times \Delta \phi=0.2 \times 0.2$ region). The central track trigger (L1CTT) does a fast track identification and momentum estimate using FPGAs and firmware. The muon system trigger (L1Muon) looks for patterns consistent with muons using hits from muon wire chambers, muon scintillation counters, and tracks from the L1CTT. The L1 forward proton detector trigger (L1FPD) selects diffractively-produced events in which the outgoing protons or antiprotons are scattered at very small angles. The trigger framework (TFW) gathers digital information from each of these L1 trigger devices and determines whether a particular event is to be accepted for further examination by L2, or rejected. The L1 trigger makes a decision in $3.5 \mu \mathrm{s}$. 


\subsubsection{The Level 2 Trigger}

The L2 trigger provides detector-specific preprocessors (L2Cal, L2Muon, L2PS, L2CTT, L2STT) and a global stage (L2Global) to refine objects coming from L1 and test for correlations in physics signatures across detector subsystems. L2 includes preprocessors for each detector subsystem and a global processor for integration of the data. Preprocessor subsystems include tracking, calorimeter, preshower, and muon systems. The subsystems work in parallel and trigger decisions are made in the L2Global stage based on physics objects reconstructed in the preprocessors.

L2 preprocessors collect data from the front-ends and L1 trigger system and analyze these data to form physics objects. L2 can also combine data across detectors to form higher quality physics objects and examine event-wide correlations in all L2 physics objects. The full detector is read out for events passing L2 and further analysis in the L3 trigger.

Data arrive at the L2 system via three transmission protocols. Calorimeter and tracker data and signals from the TFW are transmitted by $1.3 \mathrm{Gbit} / \mathrm{s}$ serial GLinks on optical fibers [37]. The muon system uses $160 \mathrm{Mbit} / \mathrm{s}$ Cypress Hotlink transmitters on coaxial cables or standard CAT/6 cables, unshielded twisted pair (UTP) Hotlinks [38].

- L2Cal The calorimeter preprocessor system identifies jets and electrons/ photons and calculates event $\not_{T}$ for the global processor. Each processor uses the 
$E_{T}$ data from the 2560 calorimeter trigger towers, including both electromagnetic towers and the electromagnetic and hadronic tower sums.

- L2Muon L2Muon receives the L1Muon output and data from approximately 150 front-end modules (from the PDTs, MDTs, and the scintillation counters). The muon candidates contain the track $p_{T}, \eta$ and $\phi$ coordinates, and quality and timing information.

- L2PS L2PS is used for electron refinement at the trigger level by providing evidence for early shower development and by giving a good spatial point for comparison with calorimeter clusters or tracks. At L2, the CPS and FPS are treated as separate detectors and their data are processed independently.

- L2STT The L2STT performs online pattern recognition in the data from the SMT. It reconstructs charged particle tracks found in the CFT at L1 with increased precision by utilizing the much finer spatial resolution of the SMT. The L2STT improves the momentum measurement of charged particle tracks at the trigger level. Requiring hits in the SMT helps reject spurious L1 triggers from accidental track patterns in the CFT. L2STT is able to measure the impact parameter of tracks precisely enough to tag the decays of long-lived particles, specifically B hadrons.

- L2CTT The L2CTT preprocessor takes inputs from the L1CTT and the 
L2STT. It operates in two different modes: i) with input tracks straight from L1CTT and ii) with input tracks from L2STT which receives input from the L1CTT and SMT barrels. The $p_{T}$ or impact parameter sorted list of L2 tracks are passed on to L2Global.

- L2Global L2Global is the first level of the trigger to examine correlations across all detector systems. L2Global is responsible for making trigger decisions based on the objects identified by the L2 preprocessors. Trigger decisions are made by creating global physics objects. L2Global imposes requirements on the global physics objects according to configuration information it receives from the Trigger Control Computer (TCC) based on the downloaded trigger menu from COOR. Level 2 Trigger Control Computer (L2TCC or TCC2) is a computer that runs L2 Relay Software (or L2RS). It provides an interface to COOR and monitor servers, and configures and controls all L2 crates.

After an L1Accept is issued, the TFW sends a trigger decision mask to L2Global, and the SCL sends notification of an L1 accepted event to every geographic sector. For each event, L2Global uses the 128 L1 trigger decision bits (each trigger decision bit represents a pass or fail of a certain trigger condition) and preprocessor inputs to make a trigger decision, and returns this decision to the TFW. The trigger list specifies which trigger conditions L2 will impose for each run. The trigger list is downloaded to L2Global by the TCC, which receives 
its instructions from COOR.

A block diagram of the L1 and L2 trigger systems is shown in Figure 3.10.

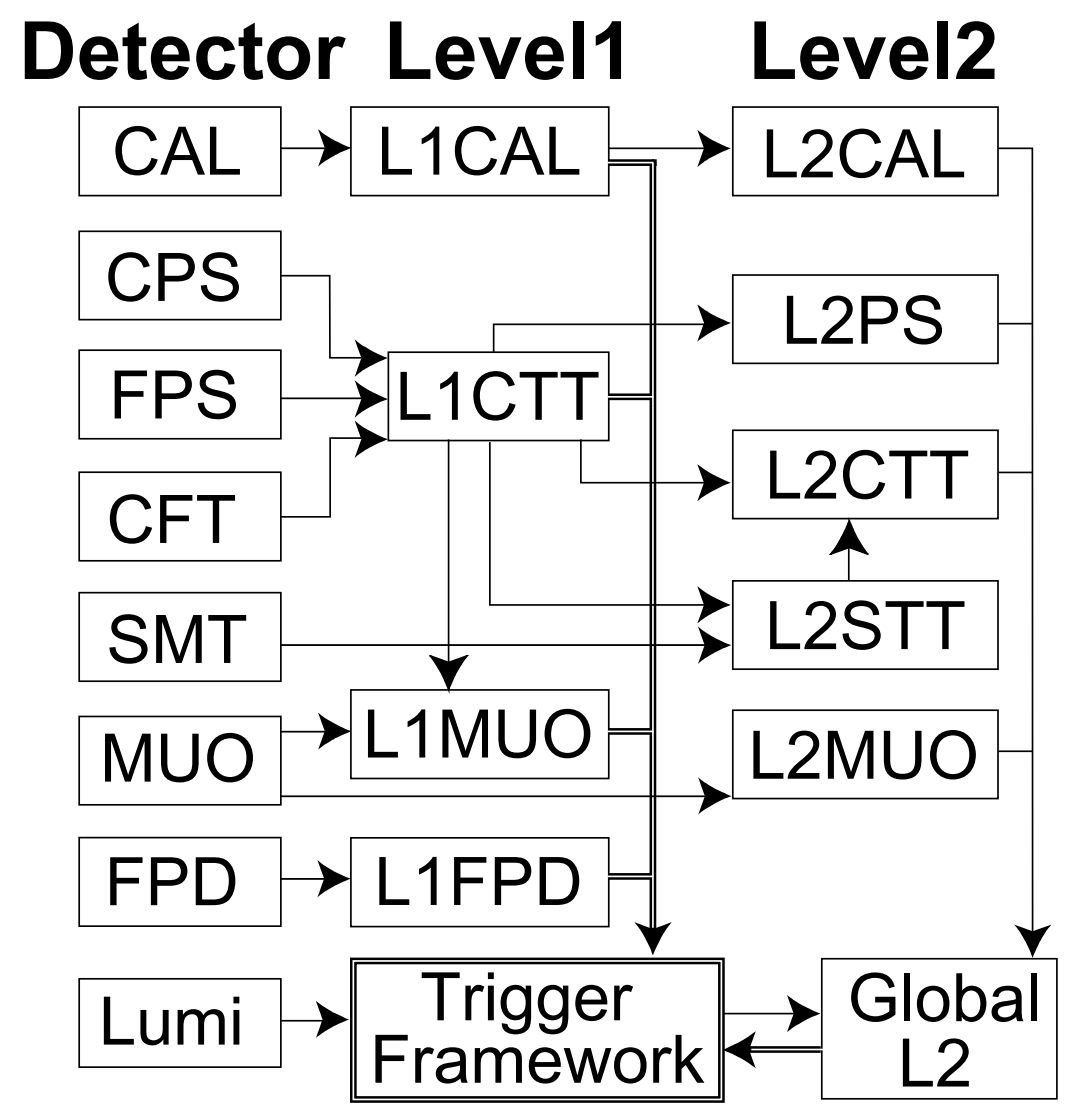

Figure 3.10: Trigger-related data flow in L1 and L2 trigger systems.

\subsubsection{The Level 3 Trigger}

The L3 trigger provides additional rejection at a higher level through a fully programmable software trigger. L3 has the full readout information available from all detector elements. L3 performs a limited reconstruction of events, reducing a nomi- 
nal $1 \mathrm{kHz}$ input rate to $50 \mathrm{~Hz}$ for offline analysis. Its decisions are based on complete physics objects as well as on the relationships between such objects. The trigger list includes blocks of filter scripts that specify one or more filters. Only when all filters in a script are satisfied, is the trigger satisfied and the event sent out to be recorded. 


\section{Chapter 4}

\section{Event Reconstruction and Object}

\section{Identification}

This chapter describes how the detector subsystems are used in order to identify physics objects, such as jets and electrons, which are created from a $p \bar{p}$ collision. Particle identification is essential for many physics analyses at the $D \emptyset$ experiment. The hits with a corresponding position and measured energy are clustered to form either a track or a calorimeter energy cluster, depending on their locations in the detector. From these physics objects are created: vertices, electrons, muons, jets and $E_{T}$. The $D \varnothing$ offline reconstruction software [39] is responsible for reconstructing those objects that are used to perform all $D \varnothing$ physics analyses.

The reconstructed objects are related to each other. By extrapolating all tracks 
to the beam line, the crossing point with the most tracks is used as the vertex. Different detector systems help in separating physics objects from each other. For example, muon will have signal at muon detector, while electron does not, which will discriminate from electron. Jets have a bigger and wider shower shape, while electron are isolated signals in calorimeter, and usually have tracks in the tracking system. MET is the sum of all energies measured in calorimeter. Since there will be some energy missing to get the balance in each direction, we assume there is a neutrino, which can not be detected. Even though physics objects are reconstructed separately, objects are not allowed to overlap and share hits or energy or tracks.

\subsection{Tracks}

Charged particles are identified in the CTT, which is discussed at Section 3.3.3. It can be used to measure the particle momentum. Matching with calorimeter energy deposits or muon hits, we can determine the particle trajectories more precisely.

In the plane perpendicular to the direction of the magnetic field, the trajectory of a charged particle is a circle. Trajectories originating from the interaction point can be characterized by two parameters $(\rho, \phi)$, where $\phi$ is the direction of the track at the point of closest approach to $(0,0)$, and the curvature:

$$
\rho=\frac{q B}{p_{t}}
$$


where $q$ is charge, $B$ is magnetic filed, and $p_{t}$ is transverse momentum.

Due to the large number of hits in the event, pattern recognition in the tracking system can not be achieved by conventional combinational approach. One must reduce the combinations of track candidates before feeding them to the final Kalman filter based local road finders, which is highly CPU consuming. In this analysis two preselection pattern recognition algorithms are used:

- Histogram Track Finding (HTF): divides the detector into slices in $(\phi, \rho)$ and uses the Hough transform [40] to preselect a initial number of combinations of groups of hits of relatively small size [41].

- Alternative Algorithm (AA): starts from combinations of hits in at least three SMT super-layers. Track candidates are extended to CFT detectors [42].

Of these two methods, AA is better at low $\mathrm{pT}$ and high impact parameter and has a lower fake rate. HTF has higher efficiency at high pT, and has higher efficiency in difficult $\eta$ regions like the overlap region (region with partial CFT coverage), and is less affected by high luminosity.

Having done these preselections, a Kalman filter based global track-finding reconstructor is applied to these track candidates to find the final global tracks in the event, and write a list of found tracks back into the event. A Kalman filter gives a weighted average of the predicted and measured track values, with more weight 
to the ones with smaller uncertainty. The estimates produced by the Kalman filter method tend to be closer to the true values than the original measurements because the weighted average has a better estimated uncertainty. As implied by their name, the global tracks are not specific to any one detector, instead to all the tracking detector subsystems (CFT, SMT, Muon).

\subsection{Primary Vertices}

The real or physics location of the hard scatter interaction point is named primary vertex (PV). See figure 4.1 for an example. In $D \varnothing$ primary vertices are reconstructed by means of an adaptive primary vertex algorithm [43]. This algorithm first selects tracks coming from different interactions by clustering them according to their $z$ position; in the second step, the location and width of the beam is determined and then used to re-fit tracks and finally each cluster of tracks is associated with a vertex using the "adaptive" technique of giving all tracks a weight and reiterating the fit; the third and last step consists of choosing the vertex that has the lowest probability of coming from a minimum bias interaction (event with no trigger requirements). 


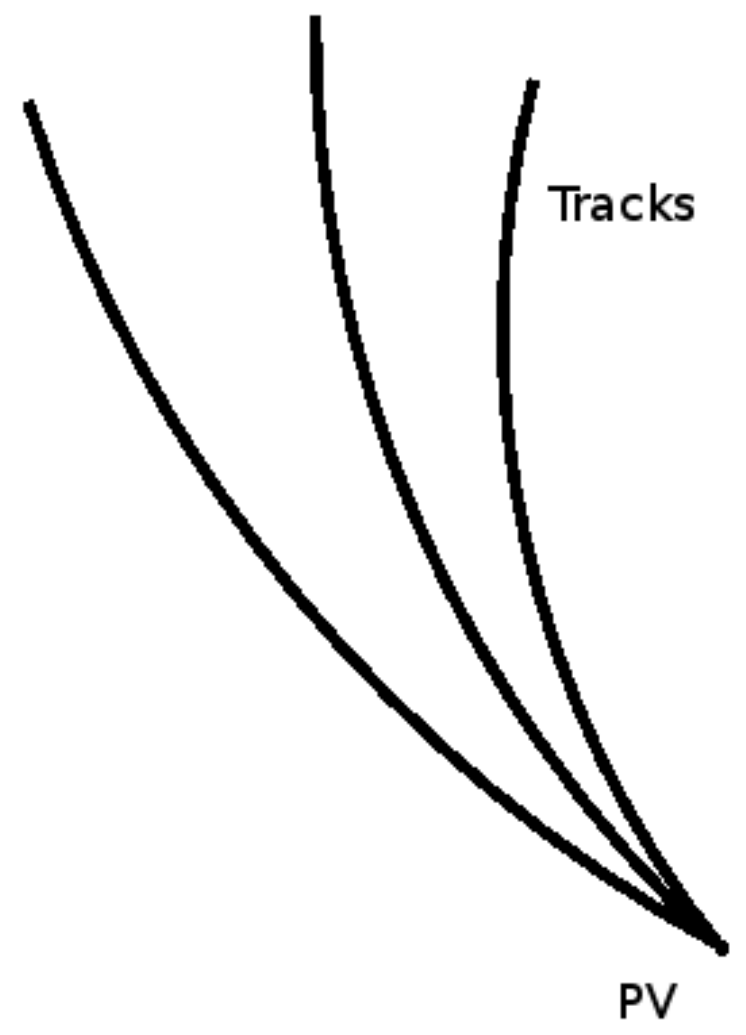

Figure 4.1: Illustration of a primary vertex. 


\subsection{Electrons}

Electrons are defined as clusters of energy depositions in the electromagnetic section of the calorimeter, matched with a track from the Central Tracking System. We

require electrons to be within the central calorimeter with $\left|\eta^{\mathrm{det}}\right|<1.1$ (CC) 3.3.2. This analysis uses three types of electrons:

\section{- Loose isolated electron}

The fraction of cluster energy deposited in the electromagnetic section of the calorimeter, over the total energy deposited in both the electromagnetic and hadronic sections of the calorimeter, is defined as the EM fraction. For electrons, we require its EM fraction $>0.9$. The electron must also meet shape requirements. The energy deposition in the calorimeter must be matched to a charged particle track from the tracking detectors with $p_{T}>5 \mathrm{GeV}$ and originating from the primary vertex. The electron is also required to be isolated (not within a jet), and the transverse momentum $p_{T}(e)>15 \mathrm{GeV}$.

\section{- Tight isolated electron}

The definition of a loose isolated electron is used as the basis for the definition of a tight isolated electron. A tight isolated electron must pass all the loose isolated electron requirements and have a value of EM-likelihood $\mathcal{L}>0.85$. Electron likelihood is the probability to discriminate a real electron from faked 
ones, using a discriminant based on several electron identification variables.

\section{- Multijets modeling electron}

A multijet electron is defined as a Loose no-track electron. It must have EM fraction $>0.9$, satisfy shape requirement, isolation $<0.2$, and $p_{T}(e)>15 \mathrm{GeV}$. There are no requirements for a matching track. We use thus defined electrons to model the multijet background sample as shown in Section 6.1.2.1.

\subsection{Muons}

Muons are identified by combining tracks in the muon spectrometer $\left(\left|\eta^{\operatorname{det}}\right|<2.0\right)$ with central detectors tracks. We use the following definitions in this analysis:

\section{- Loose isolated muon}

Loose muons must hit 3 layers in the muon section and pass the cosmic ray rejection requirements. The track reconstructed in the muon system must match a track reconstructed in the central tracker with medium track quality and match requirements. The track must be close to the primary vertex: $z$ (track, PV $)<1 \mathrm{~cm}$. The muon's transverse momentum must be $p_{T}(\mu)>15 \mathrm{GeV}$, and it must not be in a jet, determined by requiring $\Delta R(\mu$, jet $)>0.5$.

\section{- Tight isolated muon}

The definition of a loose isolated muon is used as the basis of the tight isolated 
muon. Tight isolated muons are loose muons with the additional isolation criterion that: (a) the momenta of all tracks in a cone of radius $R<0.5$ around the muon direction, except the track matched to the muon, add up to less than $20 \%$ of the muon $p_{T}$; and (b) the energy deposited in an annular cone of radius $0.1<R<0.4$ around the muon direction is less that $20 \%$ of the muon $p_{T}$.

\section{- Multijets modeling muon}

The multijet muon is defined as one which passes all selection cuts (before $b$ tagging), but no isolation or $\Delta R$ (muon, jet) requirements are made on the muon. Any jets close to the muon are removed from the event, and $E_{T} T$ is recalculated to regain momentum balance. Thus defined muons are used to model the multijet background samples.

\subsection{Jets}

A jet is a narrow cone of hadrons and other particles produced by the hadronization of a quark or gluon. Because of QCD confinement, when two quarks reach a separation distance of around $10^{-15} \mathrm{~m}$ (diameter of a hadron), they fragment into jets of mesons and baryons, that are actually recorded by the detector. The jets are measured and studied through jet reconstruction, correction and flavor tagging, in order to 
determine the properties of the original quark. Figure 4.2 shows how the quark evolves to a jet in the detector.

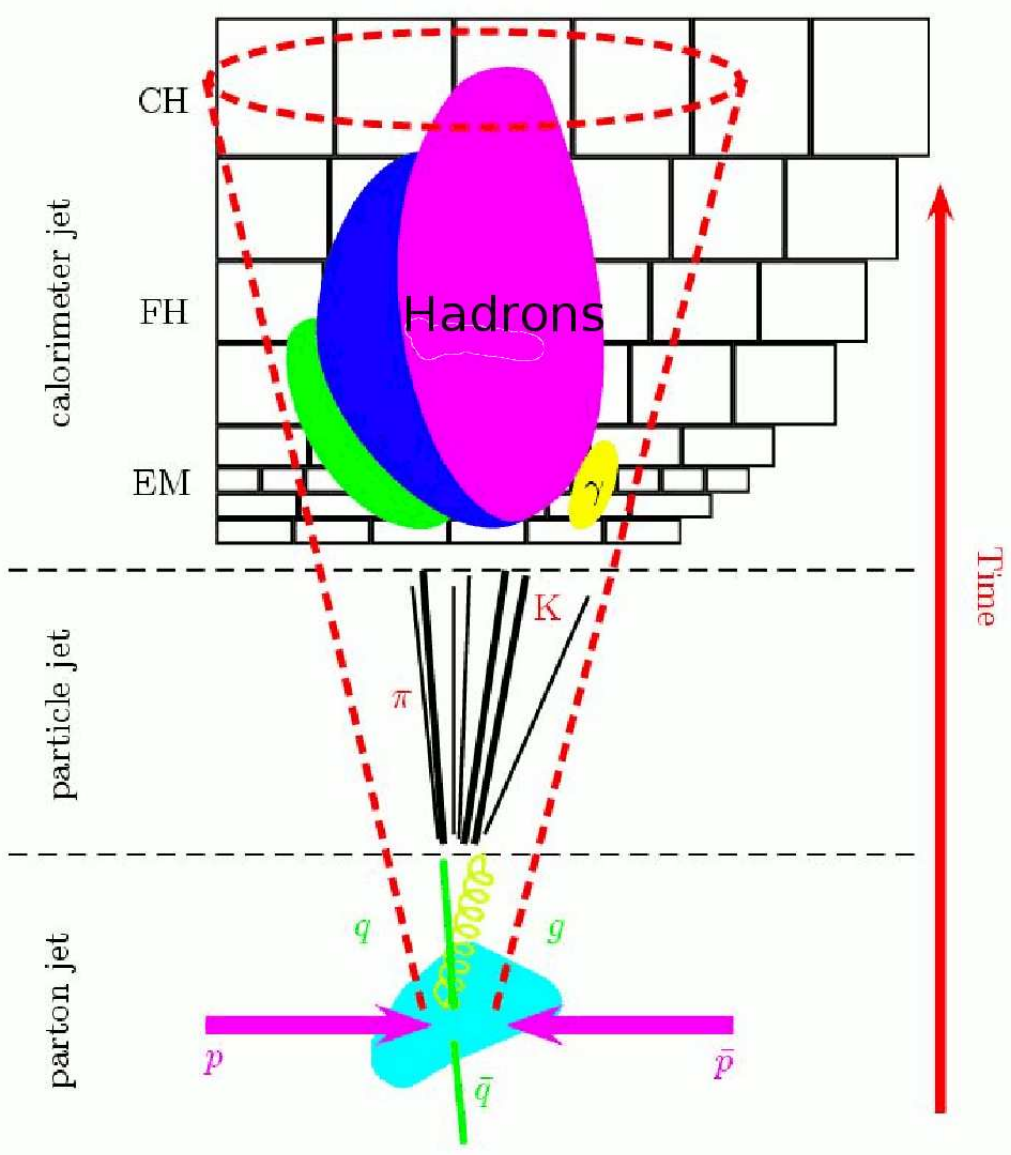

Figure 4.2: Illustration of jet formation.

In $D \varnothing$, jets are reconstructed using the RunII cone algorithm, with a cone size of 0.5. Jet energy scale corrections [44] are applied to jets in the data and MC to convert jet energies from reconstruction to particle-level. Jets containing a muon within $\Delta R(\mu$, jet-axis $)<0.5$ are considered to originate from a semileptonic $b$-quark decay 
and are corrected for the momentum carried away by the muon and the neutrino. For this correction, it is assumed that the neutrino carries the same momentum as the muon.

Monte Carlo jets need to be corrected for differences in the reconstruction and identification efficiency between data and simulation, and for different calorimeter responses. The "jet shifting, smearing, and removal algorithm" (JSSR) [45] is applied.

Jets are accepted in the analysis if they satisfy the Jet-ID selection criteria [46], and have $p_{T}>15 \mathrm{GeV}$ and $|\eta|<3.4$. For RunIIb only, we require that tracks associated with jets originate from the primary vertex.

\section{$4.6 \quad b$ Jets}

$b$ jets are jets originating from bottom quarks. They are identified ("tagged") by the b-tagging algorithm. Because top quarks nearly always decay to a bottom quark, identifying bottom quarks helps to identify the decays of top quarks. The most important feature of $b$ jets is that they have sufficient lifetime and travel some distance before decaying, resulting in a secondary vertex, as show in figure 4.3.

Given that single top quark events have at least one $b$ jet in the final state, we use a neural network (NN) based $b$ jet tagger [47] to identify jets originating from $b$ quarks. Jets are first required to be "taggable" and then are "tagged". "taggable" 


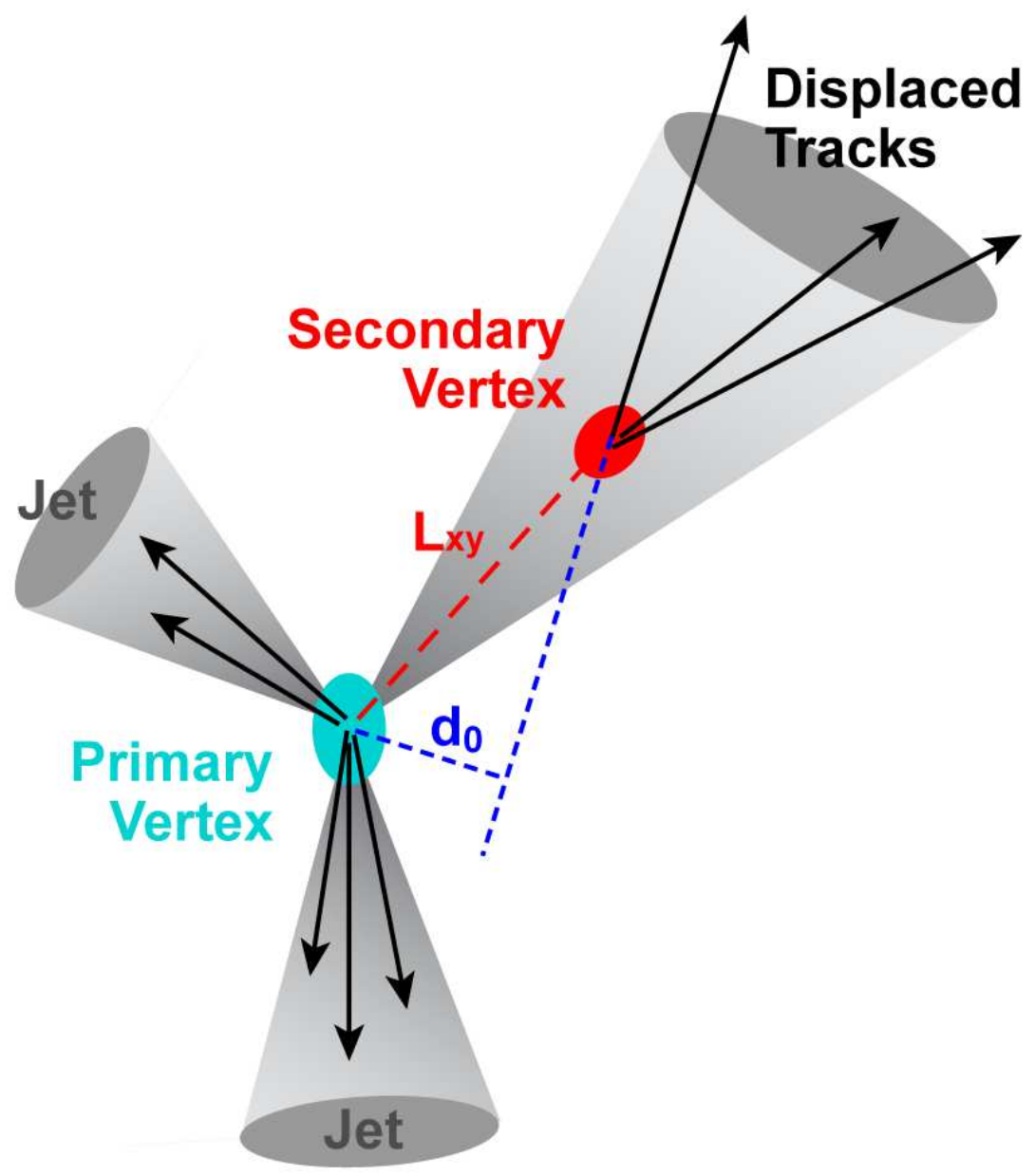

Figure 4.3: Illustration of the secondary vertex of a b jet. http://www-d0.fnal.gov/Run2Physics/top/singletop_observation/. $\quad d_{0}$ is distance between the secondary track and primary vertex. A displaced track is one with large $d_{0}$. The $2 \mathrm{D}$ distance between the primary vertex and the secondary vertex is the transverse decay length, which we denote $L_{x y}$. 
means a jet has enough tracking information that the $b$-tagging will be reliable. If a jet has no tracks, or is too forward where there is no tracker, then $b$-tagging makes no sense so we can't use it. We only try to b-tag jets which are taggable. This is done by looking at the secondary vertex information and seeing if it's consistent with a $b$ hadron decay.

Both taggability and tagging are applied directly on the Monte Carlo samples, and the efficiencies are corrected by a scale factor derived from comparing $\mathrm{MC}$ to data. We select events that either have one $b$ jet that has $b$-tag NN output $>0.775$, or two $b$ jets with $b$-tag NN output $>0.5$.

\section{7 $\quad$ Missing Transverse Energy}

In the Tevatron, the initial momentum of the colliding partons along the beam axis is unknown (parton distribution). However, the initial momentum transverse to the beam axis is zero. Even though neutrinos escape detection, the component of their momentum in the transverse plane ("missing transverse energy") can be inferred from conservation of momentum in the collision. The sum of the transverse momenta of undetected neutrinos is therefore equal to the negative sum of the transverse momenta of all particles observed in the detector. In practice, we compute the missing transverse energy by adding up vectorially the transverse energies in all cells of the electromagnetic and fine hadronic calorimeters. Cells in the coarse hadronic 
calorimeter are only added if they are part of a good jet. This raw quantity is then corrected for the energy corrections applied to the reconstructed objects, and the momenta of all muons in the event are corrected for their energy loss in the calorimeter. 


\section{Chapter 5}

\section{Event Selection}

At the Tevatron, single top quark production is a very rare process relative to its

major backgrounds (cross section $\approx 3 \mathrm{pb}^{-1}$ ). A general event selection strategy is implemented to maximize signal acceptance, and thereafter a multivariate technique is used to separate signal from background, which will be discussed in the next chapter.

\section{$5.1 \quad$ Strategy}

The experimental signal for single top events is one isolated high transverse momentum central lepton, and missing transverse energy from the decay of $W$ boson from the top quark decay, accompanied by a $b$ jet from the top decay, and a second jet which is sometimes also from the decay of a $b$ hadron. Since there may be significant 
initial- or final- state radiation, we include our search events with between two to four jets.

The composition of the background components is different for events with dif-

ferent jet multiplicities $(2,3,4)$ and lepton flavors (electron, muon). The signal to background ratio and signal acceptance in each subsample set also differ significantly. Thus the data is divided into many orthogonal sub-samples (i.e. channels) according to jet and $b$-tag multiplicities. The event selection criteria in this analysis are applied separately for the different channels, and then several channels are combined to increase statistics.

\subsection{Event selection cuts}

We apply a loose event selection to find events containing an isolated lepton, missing transverse energy, and two to four jets with high transverse momentum. Then we make a series of topological cuts to reduce the multijet background in each channel to an acceptable low level.

\subsubsection{General selection}

These selection cuts are applied for both electron and muon channels.

- Good quality (for data). 
- Instantaneous luminosity $>0$.

- Pass Mega-OR trigger requirement, in which at least one of the selected "reasonable" triggers has to fire. For details of which triggers are included in the Mega-OR trigger and their efficiencies, see Appendix ??.

- Good primary vertex: $\left|z_{\mathrm{PV}}\right|<60 \mathrm{~cm}$ with at least three tracks attached.

- Two, three, or four good jets with $p_{T}>15 \mathrm{GeV}$ and $\left|\eta^{\text {det }}\right|<3.4$. For Run IIb jets are required to be vertex-confirmed.

- The leading jet $p_{T}>25 \mathrm{GeV}$.

- For Run IIb only, the second leading jet $p_{T}$ cut is raised to $20 \mathrm{GeV}$ in events with exactly three good jets.

- For Run IIb only, the second leading jet $p_{T}$ cut is raised to $25 \mathrm{GeV}$ in events with exactly four good jets.

- Jet triangle cut of $\mid \Delta \phi\left(\right.$ leading jet, $\left.\mathbb{E}_{\mathrm{T}}\right) \mid$ vs. $\mathbb{E}_{T}$ :

- $|\Delta \phi|$ from 1.5 to $\pi \mathrm{rad}$ when $E_{T}=0 \mathrm{GeV}$, and $E_{T}$ from 0 to $35 \mathrm{GeV}$ when $\mid \Delta \phi=\pi \mathrm{rad}$.

- Missing transverse energy

- $20<$ E $_{T}<200 \mathrm{GeV}$ in events with exactly two good jets. 
- $25<\not_{T}<200 \mathrm{GeV}$ in events with three or more good jets.

- Scalar sum of the transverse energies of all good jets

- $H_{T}$ (alljets) $>50(55) \mathrm{GeV}$ in events with exactly two good jets for Run IIa (Run IIb) period.

- $H_{T}$ (alljets) $>75(80) \mathrm{GeV}$ in events with exactly three good jets for Run IIa (Run IIb) period.

- $H_{T}$ (alljets) $>100$ (110) $\mathrm{GeV}$ in events with exactly four good jets for Run IIa (Run IIb) period .

- Jet triangle cut of second leading jet $p_{T}$ vs. $H_{T}$ (alljets) for Run IIb in events with exactly two good jets.

- second leading jet $p_{T}$ from 0 to $27.5 \mathrm{GeV}$ when $H_{T}$ (alljets) $=0 \mathrm{GeV}$, and $H_{T}$ (alljets) from 0 to $165 \mathrm{GeV}$ when second leading jet $p_{T}=0 \mathrm{GeV}$.

- Scalar sum of the transverse energies of all good jets, the lepton transverse momentum, and the missing transverse energy

- $H_{T}$ (alljets, lepton, $\left.E_{T}\right)>120 \mathrm{GeV}$ in events with exactly two good jets.

- $H_{T}$ (alljets, lepton, $\left.\not{ }_{T}\right)>140 \mathrm{GeV}$ in events with exactly three good jets.

- $H_{T}$ (alljets, lepton, $\left.\not E_{T}\right)>160 \mathrm{GeV}$ in events with exactly four good jets. 


\subsubsection{Electron channel selection}

These selection cuts are applied for electron channels only.

- Only one tight electron with $\left|\eta^{\text {det }}\right|<1.1$ and $p_{T}>15(20) \mathrm{GeV}$ in events with 2 (3 or more) good jets.

- No additional loose electron with $p_{T}>15 \mathrm{GeV}$.

- No tight isolated muon with $p_{T}>15 \mathrm{GeV}$ and within $\left|\eta^{\text {det }}\right|<2.0$.

- Electron coming from the primary vertex: $|\Delta z(e, \mathrm{PV})|<1 \mathrm{~cm}$.

- Electron triangle cuts of $\left|\Delta \phi\left(e, \not_{T} T\right)\right|$ vs. $E^{\prime} T$

- $|\Delta \phi|$ from 2 to $0 \mathrm{rad}$ when $\mathscr{E}_{T}=0 \mathrm{GeV}$, and $\not_{T}$ from 0 to $40 \mathrm{GeV}$ when $|\Delta \phi|=0 \mathrm{rad}$.

- $|\Delta \phi|$ from 1.5 to $0 \mathrm{rad}$ when $E_{T}=0 \mathrm{GeV}$, and $\not_{T}$ from 0 to $50 \mathrm{GeV}$ when $|\Delta \phi|=0 \mathrm{rad}$.

- $|\Delta \phi|$ from 2 to $\pi \mathrm{rad}$ when $E_{T}^{\prime}=0 \mathrm{GeV}$, and $\not_{T}$ from 0 to $24 \mathrm{GeV}$ when $|\Delta \phi|=\pi \mathrm{rad}$.

\subsubsection{Muon channel selection}

These selection cuts are applied for muon channels only.

- Only one tight muon with $p_{T}>15 \mathrm{GeV}$ and $\left|\eta^{\text {det }}\right|<2.0$. 
- No additional loose muons with $p_{T}>4 \mathrm{GeV}$.

- No loose electron with $p_{T}>15 \mathrm{GeV}$ and within $\left|\eta^{\operatorname{det}}\right|<2.5$.

- Muon coming from the primary vertex: $|\Delta z(\mu, \mathrm{PV})|<1 \mathrm{~cm}$.

- Muon triangle cuts of $\left|\Delta \phi\left(\mu, \mathbb{E}_{\mathrm{T}}\right)\right|$ vs. $\not_{T}$

- $|\Delta \phi|$ from 1.2 to 0 rad when $\not_{T}=0 \mathrm{GeV}$, and $\not_{T}$ from 0 to $85 \mathrm{GeV}$ when $|\Delta \phi|=0 \mathrm{rad}$.

- $|\Delta \phi|$ from 2.5 to $\pi \mathrm{rad}$ when $E_{T}=0 \mathrm{GeV}$, and $\not_{T}$ from 0 to $30 \mathrm{GeV}$ when $|\Delta \phi|=\pi \mathrm{rad}$.

- Muon track curvature significance cuts of $\mid$ TrackCurvSig $\mid$ vs. $\left|\Delta \phi\left(\mu, \not E_{T}\right)\right|$, where $\mid$ TrackCurvSig $|=| \frac{q / p_{T}}{\sigma\left(1 / p_{T}\right)} \mid$, and $q$ and $p_{T}$ are the charge and transverse momentum of the charged track associated with the muon.

- $|\Delta \phi|$ from $0.875 \pi$ to $\pi$ rad when |TrackCurvSig $\mid=0$, and |TrackCurvSig| from 0 to 4 (6) when $|\Delta \phi|=\pi$ rad for Run IIa (Run IIb) period.

- $|\Delta \phi|$ from 2 to $\pi$ rad when $\mid$ TrackCurvSig $\mid=0$, and |TrackCurvSig $\mid$ from 0 to 2 (3) when $|\Delta \phi|=\pi$ rad for Run IIa (Run IIb) period.

These cuts are needed to reject events with poorly measured muons that cause an excess in data over background model in the $\Delta \phi$ distributions. 
- Transverse momentum of the leading jet within the ICD region of the detector: leading jet $p_{T}>30 \mathrm{GeV}$ when $1.0<\mid$ leading jet $\eta^{\text {det }} \mid<1.5$.

- Transverse momentum of the second leading jet within the ICD region of the detector: leading jet $p_{T}>25 \mathrm{GeV}$ when $1.0<\mid$ leading jet $\eta^{\text {det }} \mid<1.5$ only for Run IIb period with exactly three good jets.

\subsection{4 b-tagging selection}

To be able to extract single top events we also look for the presence of one or two $b$ tagged jets. We select events that have exactly one jet with $b$-tag NN output $>0.775$ and no other jet with $b$-tag NN output $>0.5$, or two jets with $b$-tag NN output $>$ 0.5. This ensures that there is no overlap between the one tag and two tags samples. 


\section{Chapter 6}

\section{Data and Simulation Samples}

In this chapter, the data and simulation samples used for this analysis are presented. The corrections to multijet and MC simulation samples are discussed.

\subsection{Data samples}

The data sample was collected from the $p \bar{p}$ collisions in the Tevatron at a center of mass energy of $1.96 \mathrm{TeV}$, using the $D \varnothing$ detector.

\subsubsection{Signal data samples}

The data sample was collected between August 2002 and February 2006 (Run IIa) and between June 2006 and June 2009 (RunIIb), corresponding to $1.08+4.28=$ 
$5.35 \mathrm{fb}^{-1}$ of good quality data in each of the $e$ and $\mu$ channels, as shown in Table 6.1. The datasets used in this analysis are listed in Appendix B.

Table 6.1: Integrated luminosity for the datasets used in this analysis.

\begin{tabular}{cc||c} 
Data sample & Trigger Version & Integrated Luminosity $\left[\mathrm{pb}^{-1}\right]$ \\
\hline Run IIa & v8 - v14 & 1078.81 \\
\hline Run IIb & v15a & 534.44 \\
& v15b & 688.02 \\
& v15c & 397.31 \\
& v16 & 2661.90 \\
\hline Total & v8-16 & 5360.48
\end{tabular}

We require events to pass the Mega-OR trigger requirement, in which at least one of the selected "reasonable" triggers has to fire. We measure that the Mega-OR trigger efficiency for the events that pass our selection is $100 \%$ within the assigned systematic uncertainties and thus no additional trigger efficiency correction factors are needed in our MC samples. Details of trigger efficiencies measurement are described in Appendix ??.

\subsubsection{Multijets data samples}

Multijet events form the second largest background after $W+$ jets. We use data events to model it. We select those events that pass all our event selection criteria 
except the lepton requirements.

\subsubsection{Multijets in the electron channel}

The multijets background in the electron channel is modeled with data events that pass all selection cuts (before $b$ tagging) except that the EM object is not required to have a matching track. In addition, the EM object is required to fail the electron likelihood cut.

This sample suffers from a kinematic bias that can be corrected by reweighting the events with a kinematic correction factor [48]. The kinematic correction factor is defined as

$$
\mathrm{QCDWeight}=\varepsilon_{\text {fake }-e} /\left(1-\varepsilon_{\text {fake }-e}\right)
$$

which depends on the parameter $\varepsilon_{\text {fake- }} e$, which is the efficiency for a multijet faked electron (See definition in Section 4.3.) to pass the tight electron criteria.

$\varepsilon_{\text {fake }-e}$ is measured as the ratio of the number of events with a tight electron over the number of events with an multijet faked electron in the $E_{T}<10 \mathrm{GeV}$ region. Even though the low- $\not_{T}$ region is dominated by multijet events, there is a nonnegligible contamination from real electrons, in particular for the tight sample. This signal contamination originates mostly from $Z+$ jets events and to some extent also from $W+$ jets events. The expected contributions to the multijet and tight electron samples originating from $W+$ jets and $Z+$ jets events are estimated from Monte Carlo 
and subtracted from the number of events in data before computing $\varepsilon_{\text {fake}}-e$.

\subsubsection{Multijets in the muon channel}

The multijets background in the muon channel is modeled with data events that pass all selection cuts (before $b$ tagging), but no isolation or $\Delta R$ (muon, jet) requirements are made on the muon. In order to make the jets in these events match those in the signal data, any jets close to the muon are removed from the event, and $\not_{T}$ is recalculated to regain momentum balance.

\subsection{Simulation samples}

\subsubsection{Signal simulation samples}

Simulated single top event samples are generated with the CompHEP-SINGLETOP [49] Monte Carlo event generator. SINGLETOP produces events whose kinematic distributions match those from NLO calculations. The top quark mass is $172.5 \mathrm{GeV}$, and this applies to all top quark samples. The PDF set is CTEQ6M [50] and the scales

are $m_{t}^{2}$ for the $s$-channel and $\left(m_{t} / 2\right)^{2}$ for the $t$-channel. The top quarks and the $W$ bosons from the top quark decays are decayed in CompHEP-SINGLEToP to ensure the spins are properly transferred. PYTHIA [51] is used to add the underlying event and initial- and final-state radiation. EVTGEN [52] is used to decay $b$ hadrons. 


\subsubsection{Background simulation samples}

The $W+$ jets, $Z+$ jets, and $t \bar{t}$ background samples for this analysis are generated using ALPGEN [53] (version 2.11). Zero-bias overlay events are used to better model the high instantaneous luminosity. All MC simulated events have an additional number of zero bias $p \bar{p}$ events superimposed, dependent on the luminosity profile.

Table 6.2 shows the cross sections, branching fractions, and initial numbers of events for the Monte Carlo samples. The $W+$ jets and $Z+$ jets cross sections are from ALPGEN.

\subsubsection{Simulation samples corrections and normalization}

The expected number of Monte Carlo events coming from the simulated backgrounds samples are obtained in three steps:

- First, apply scale factors such that the MC reconstruction and selection efficiencies match those found in data, as explained in Section 6.2.3.1 below.

- Second, the $t \bar{t}, Z+$ jets, dibosons, and single top samples are normalized to the integrated luminosity of our dataset using the cross sections and branching fractions listed in Table 6.2. The normalization weight is calculated as:

$$
w=\frac{\sigma_{\text {Theory }} \times \text { Integrated Luminosity }}{\text { Number of events produced by MC }} .
$$


Table 6.2: The cross sections, branching fractions, and initial numbers of events in the Monte Carlo event samples. The symbol $\ell$ stands for electron plus muon plus tau decays. We only include tau decays to leptons.

\begin{tabular}{|c|c|c|c|c|}
\hline \multirow[b]{2}{*}{ Event Type } & \multicolumn{4}{|c|}{ The Monte Carlo Event Sets } \\
\hline & $\begin{array}{c}\text { Cross Section } \\
{[\mathrm{pb}]}\end{array}$ & $\begin{array}{l}\text { Branching } \\
\text { Fraction }\end{array}$ & $\begin{array}{l}\text { No. of p17 } \\
\text { Events }\end{array}$ & $\begin{array}{c}\text { No. of p20 } \\
\text { Events }\end{array}$ \\
\hline \multicolumn{5}{|l|}{ Signals } \\
\hline$t b \rightarrow \ell+\mathrm{jets}$ & $1.04_{-0.04}^{+0.04}$ & $0.324 \pm 0.003$ & $0.6 \mathrm{M}$ & $0.5 \mathrm{M}$ \\
\hline$t q b \rightarrow \ell+$ jets & $2.26_{-0.12}^{+0.12}$ & $0.324 \pm 0.003$ & $0.6 \mathrm{M}$ & $0.5 \mathrm{M}$ \\
\hline Signal total & $3.30_{-0.16}^{+0.16}$ & $0.324 \pm 0.003$ & $1.2 \mathrm{M}$ & $1.0 \mathrm{M}$ \\
\hline \multicolumn{5}{|l|}{ Backgrounds } \\
\hline$t \bar{t} \rightarrow \ell+$ jets & $7.46_{-0.67}^{+0.48}$ & $0.438 \pm 0.004$ & $1.5 \mathrm{M}$ & $1.3 \mathrm{M}$ \\
\hline$t \bar{t} \rightarrow \ell \ell$ & $7.46_{-0.67}^{+0.48}$ & $0.105 \pm 0.001$ & $1.5 \mathrm{M}$ & $1.3 \mathrm{M}$ \\
\hline Top pairs total & $7.46_{-0.67}^{+0.48}$ & $0.543 \pm 0.005$ & $3.0 \mathrm{M}$ & $1.6 \mathrm{M}$ \\
\hline$W b \bar{b} \rightarrow \ell \nu b b$ & 90.5 & $0.324 \pm 0.003$ & $2.7 \mathrm{M}$ & $3.0 \mathrm{M}$ \\
\hline$W c \bar{c} \rightarrow \ell \nu c c$ & 260 & $0.324 \pm 0.003$ & $2.7 \mathrm{M}$ & $3.0 \mathrm{M}$ \\
\hline$W j j \rightarrow \ell \nu j j$ & 23,831 & $0.324 \pm 0.003$ & $55 \mathrm{M}$ & $97 \mathrm{M}$ \\
\hline $\mathrm{W}+$ jets total & 24,182 & $0.324 \pm 0.003$ & $60.4 \mathrm{M}$ & $103 \mathrm{M}$ \\
\hline$Z b \bar{b} \rightarrow \ell \ell b b$ & 38.7 & $0.0337 \pm 0.0000$ & $0.7 \mathrm{M}$ & $0.7 \mathrm{M}$ \\
\hline$Z c \bar{c} \rightarrow \ell \ell c c$ & 106 & $0.0337 \pm 0.0000$ & $0.7 \mathrm{M}$ & $0.7 \mathrm{M}$ \\
\hline$Z j j \rightarrow \ell \ell j j$ & 7,032 & $0.0337 \pm 0.0000$ & $14 \mathrm{M}$ & $4.0 \mathrm{M}$ \\
\hline $\mathrm{Z}+$ jets total & 7,177 & $0.0337 \pm 0.0000$ & $15.4 \mathrm{M}$ & $5.4 \mathrm{M}$ \\
\hline$W W \rightarrow$ anything & $11.6 \pm 0.4$ & $1.0 \pm 0.0$ & $2.0 \mathrm{M}$ & $0.7 \mathrm{M}$ \\
\hline$W Z \rightarrow$ anything & $3.25 \pm 0.11$ & $1.0 \pm 0.0$ & $1.0 \mathrm{M}$ & $0.6 \mathrm{M}$ \\
\hline$Z Z \rightarrow$ anything & $1.33 \pm 0.04$ & $851.0 \pm 0.0$ & $1.0 \mathrm{M}$ & $0.5 \mathrm{M}$ \\
\hline Diboson total & $16.2 \pm 0.6$ & $1.0 \pm 0.0$ & $4.0 \mathrm{M}$ & $1.8 \mathrm{M}$ \\
\hline
\end{tabular}


- Finally, the $W+$ jets background is corrected for the reconstruction efficiencies, but normalized to the data before tagging, as explained in Section 6.2.3.2 below.

\subsubsection{Simulation efficiencies corrections}

The efficiencies to reconstruct, identify and select objects in the MC are higher than in data, so the following scale factors are used to correct for those differences:

\section{- Primary vertex position reweighting}

We reweight the position of the primary vertices along the beamline in $z$ to make the distribution in $\mathrm{MC}$ events match data.

\section{- Instantaneous luminosity reweighting}

We reweight the instantaneous luminosity distributions of the MC events to match those in Run IIa and Run IIb data.

\section{- $\mathbf{Z} p_{T}$ distribution reweighting}

We reweight the inclusive $Z$ boson $p_{T}$ spectrum to match the theory model.

\section{- EM ID efficiency correction factor}

We correct each event in the electron channel with a scale factor that accounts for the differences in electron cluster finding and identification efficiency [54] [55]. For multijet background model electrons, the $\mathrm{MC}$ to data scale factor is consistent with one. 


\section{- Muon ID and isolation efficiency correction factor}

We correct each event in the muon channel for the muon ID efficiency, the track match, and the isolation. The ID scale factor is parametrized in $\left(\eta_{\mathrm{det}}, \phi\right)$; track match is parametrized in track- $z$ and $\eta_{\mathrm{CFT}}$; and the isolation in $\eta$. The

parametrizations derived in $Z \rightarrow \mu \mu$ data and MC, as described in Ref. [56]: There is an additional mismodeling correction applied to the muon-ID as a function of $\eta_{\text {det }}$ in $W+$ jets events.

- Jet shifting, smearing, and removal (JSSR)

We use the JSSR processor [45] for the $W+$ jets and $Z+$ jets samples (including $W b b, W c c, Z b b$ and $Z c c)$, and dibosons.

\section{- Taggability and $b$-tagging efficiency correction factor}

The taggability and tagging algorithms are directly applied to both data samplesand the simulated samples (direct tagging), and the efficiencies corrected by a scale factor derived from comparing MC to data. Random tagging is used for $W+$ light and $W+c$ samples to ensure proper MC statistics after tagging. The random tagging algorithm replaces the standard b-tag NN output with one that is chosen randomly between a probability $p=1 / 2$ and $p=1 / 24$. This causes many more of the $W+$ light jet events to fall into the one and two tagged samples. 


\subsubsection{2 $W+$ jets and multijets backgrounds}

The $W+$ jets background is taken from Monte Carlo samples and corrected for reconstruction efficiencies as described in Section 6.2.3.1 above. The samples are weighted by the leading-log (LL) cross sections (including branching fractions) from ALPGEN and the integrated luminosity. Comparisons of LL and NLO calculations to data [57] show the need for $K^{\prime}$-factors to scale ALPGEN cross sections for $W j j, W c j$ ( $j$ represents light partons, including $u, d, s), W b \bar{b}$, and $W c \bar{c}$, and also $K_{H F}^{\prime}$-factors for $W b \bar{b}$ and $W c \bar{c}$ ( $H F$ represents Heavy Flavor partons, which include $b$ and $c$ ). Similar cross section correction is done to the $Z+$ Jets samples. The flavor composition of $W+$ jets and $Z+$ jets samples are normalized to NLO.

The multijets background is modeled with the orthogonal data samples described in Section 6.1.2. The multijets and the $W+$ jets backgrounds are normalized using an iterative Kolmogorov-Smirnov-test normalization method (IKS).

In the IKS method, two anti-correlated scale factors are applied to the $W+$ jets and multijets samples to get the proper normalization to data. The scale factors are determined by measuring the agreement between data and background model on three distributions sensitive to the $W+$ jets and multijets normalizations: lepton $p_{T}, \not E_{T}$, and $W$ transverse mass. The final $W+$ jets and multijets scale factors are the ones that maximize the product of the Kolmogorov-Smirnov (KS) test values of each distribution. The KS-test tries to determine if two datasets differ significantly. 
It has the advantage of making no assumption about the distribution of data.

In order to deal with the mismodeling observed for some angular variables, the $W+$ jets and $Z+$ jets samples are reweighted to reproduce the jet $\eta$ distributions in data. The reweighting is done by comparing the $W+$ jets and $Z+$ jets samples to data after subtraction of all other backgrounds. It is done successively on the leading and second leading jet $\eta$, the $\Delta R$ between them, and on the third and fourth jet $\eta$ when applicable. The reweighting is done with a fitted function that excludes the ICR region and preserves the overall normalization.

It is found that the normalization is preserved after applying $b$-tagging, and no further IKS normalization is needed.

\subsection{Lepton charge mis-ID measurement samples}

Charge measurement is important for this analysis since the $\mathrm{CP}$ violation can be diluted by the mis-identification (mis-ID) of the lepton charge. We rely on the track charge to determine whether the selected lepton carries positive or negative charge.

We select $Z \rightarrow e e$ or $Z \rightarrow \mu \mu$ events to measure the charge mis-ID rate. For this purpose, we use samples of events which have two electrons or muons with high transverse momentum in them. The charge mis-ID rate is defined as the ratio between the number of same sign events and the total number of di-lepton events. We make the same lepton and jet requirements to select this sample as in the single 
top analysis. We make no MET cut.

Details of the sample definitions can be found in Appendix D. 


\section{Chapter 7}

\section{Event Yields and Data/Simulation}

\section{Comparison}

In this chapter we present data and simulation sample event yields after the event selection described in Chapter 5.

\section{$7.1 \quad$ Event yields}

The samples after selection, which we call "pretagged", "onetagged" and "twotagged" samples, are dominated by $W+$ jets events, with some $t \bar{t}$ contributions that become more significant for higher jet multiplicities, and smaller contributions from multijets, $Z+$ jets and diboson events. Figure 7.1 gives a pie chart illustration of the composition 


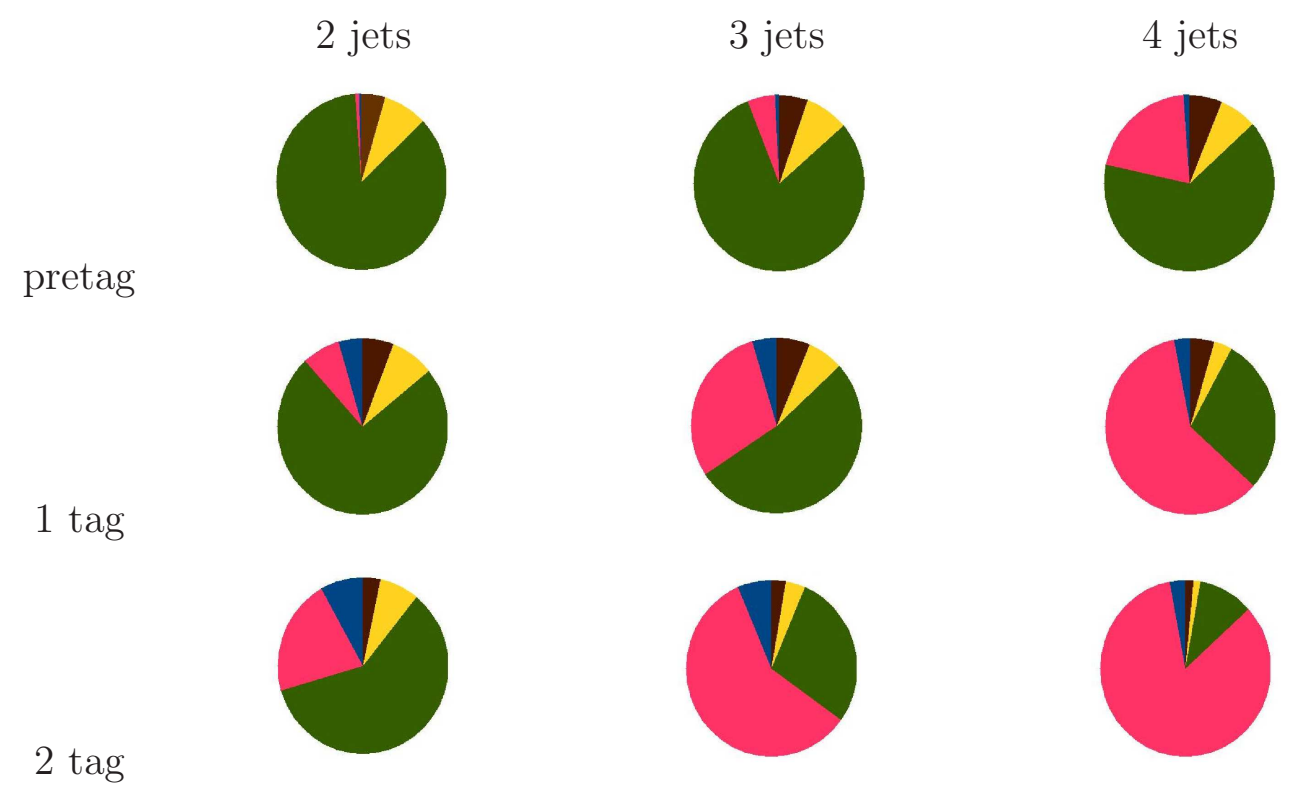

Figure 7.1: A pie chart illustration of the components of each background and signal sample in each jet channel in pretag, one tag, and two tag bins.

of each background and signal sample in each jet channel after this selection.

We use the term "yield" to mean the number of events of the signal or background in question predicted to be in the nearly $5.4 \mathrm{fb}^{-1}$ of data analyzed here. Thus, the event yield for a specific sample is defined as:

$$
\mathcal{Y}=\sigma \times \mathcal{L}=\sigma \times 5.4 \mathrm{fb}^{-1}
$$

where, $\sigma$ is the cross section for that sample, and $\mathcal{L}=5.4 \mathrm{fb}^{-1}$ is the integrated luminosity used in this analysis. On the other hand, the event yield for a MC sample 
can also be expressed as:

$$
\mathcal{Y}=\sum_{i}^{\mathcal{N}_{\text {selected }}} \varepsilon(i)_{\text {corrections }}
$$

where $\mathcal{N}_{\text {selected }}$ is the number of events remaining after selection in that MC sample, and $\varepsilon(i)_{\text {corrections }}$ is the overall efficiency correction factor for the $i$ th event after selection to account for differences between data and MC from particle ID, vertex confirmation, taggability and $b$-tagging, that is:

$$
\begin{aligned}
\varepsilon(i)_{\text {corrections }}= & \varepsilon(i)_{\text {particle ID }} \times \varepsilon(i)_{\text {vertex confirmation }} \times \\
& \varepsilon(i)_{\text {taggability }} \times \varepsilon(i)_{\text {b-tagging }} \times \frac{\sigma}{N_{M C}}
\end{aligned}
$$

, where $\sigma$ is cross section and $N_{M C}$ total number of events in MC sample. For data, the event yield is equal to the number of events.

Tables 7.1 and 7.2 show the yields for all signals and backgrounds for events with one and two b-tagged jets respectively.

For the CP violation measurement, an extra cut applied is to split the samples by lepton charge. Tables 7.3 and 7.4 show these yields for all signals and backgrounds after splitting samples by lepton charge ("positive" and "negative" samples). The sum of the positive and negative yields is identical to the total. We do not redo the normalization of $W+$ jets and QCD separately for positive and negative samples. 
From these tables we can see that in all of the sum of yields of all MC samples equal to the number of events in data. $b$ tagging improves signal to background ratio radically, however, we also lose event statistics in the 1-tag and 2-tag samples. Note that the yield values shown in these and subsequent tables have been rounded for clarity, so that the sums of the components do not always equal exactly the values given for these sums, however all calculations have been done with full-precision values.

Table 7.1: One $\mathrm{b}$ tagged event yields with statistical uncertainty for each jet multiplicity and for all analysis channels combined (Electron+Muon, Run IIa+Run IIb).

\begin{tabular}{l||ccc|c} 
Source & 2 jets & 3 jets & 4 jets & All Channels \\
\hline \begin{tabular}{l|c} 
Signals \\
$t b+t q b$
\end{tabular} & & & & \\
Backgrounds & & & & \\
$t \bar{t}$ & $313 \pm 1.8$ & $545 \pm 2.8$ & $499 \pm 2.8$ & $1,356 \pm 4.4$ \\
$W+$ jets & $3,222 \pm 20$ & $961 \pm 9.9$ & $239 \pm 4.0$ & $4,421 \pm 23$ \\
$Z+$ jets \& dibosons & $357 \pm 6.8$ & $123 \pm 4.3$ & $29 \pm 1.7$ & $510 \pm 8.2$ \\
Multijets & $258 \pm 2.2$ & $117 \pm 1.9$ & $37 \pm 0.83$ & $412 \pm 3.1$ \\
Background Sum & $4,150 \pm 22$ & $1,745 \pm 11$ & $804 \pm 5.2$ & $6,699 \pm 25$ \\
Backgrounds+Signals & $4,348 \pm 22$ & $1,830 \pm 11$ & $830 \pm 5.3$ & $7,008 \pm 25$ \\
Data & $4,284 \pm 65$ & $1,772 \pm 42$ & $851 \pm 29$ & $6,907 \pm 83$ \\
$S: B$ & $1: 21$ & $1: 21$ & $1: 31$ & $1: 22$ \\
\hline
\end{tabular}


Table 7.2: Two b tagged event yields with statistical uncertainty for each jet multiplicity and for all analysis channels combined (Electron+Muon, Run IIa+Run IIb).

\begin{tabular}{l||ccc|c} 
Source & 2 jets & 3 jets & 4 jets & All Channels \\
\hline Signals & & & & \\
$\quad t b+t q b$ & $46 \pm 0.37$ & $31 \pm 0.34$ & $13 \pm 0.22$ & $90 \pm 0.55$ \\
Backgrounds & & & & \\
$t \bar{t}$ & $121 \pm 0.97$ & $285 \pm 1.8$ & $361 \pm 2.2$ & $767 \pm 3.0$ \\
$W+$ jets & $338 \pm 5.1$ & $139 \pm 3.2$ & $45 \pm 1.7$ & $522 \pm 6.3$ \\
$Z+$ jets \& dibosons & $42 \pm 1.7$ & $18 \pm 1.1$ & $5.9 \pm 0.53$ & $66 \pm 2.1$ \\
Multijets & $19 \pm 0.62$ & $13 \pm 0.64$ & $6.3 \pm 0.33$ & $39 \pm 0.95$ \\
Background Sum & $520 \pm 5.5$ & $456 \pm 3.9$ & $418 \pm 2.8$ & $1,394 \pm 7.3$ \\
Backgrounds+Signals & $566 \pm 5.5$ & $487 \pm 3.9$ & $431 \pm 2.8$ & $1,484 \pm 7.3$ \\
Data & $597 \pm 24$ & $535 \pm 23$ & $432 \pm 21$ & $1,564 \pm 40$ \\
$S: B$ & $1: 11$ & $1: 15$ & $1: 33$ & $1: 16$ \\
\hline
\end{tabular}


Table 7.3: Number of expected yields and observed data counts in "positive" samples after $b$-tagging.

\begin{tabular}{l||ccc|c} 
Source & 2 jets & 3 jets & 4 jets & All Channels \\
\hline \multirow{2}{*}{$t b$} & $53 \pm 7.6$ & $22 \pm 3.7$ & $6.4 \pm 1.7$ & $81 \pm 13$ \\
$t q b$ & $70 \pm 5.6$ & $37 \pm 4.4$ & $13 \pm 2.9$ & $119 \pm 13$ \\
$t b+t q b$ & $122 \pm 13$ & $59 \pm 8.1$ & $19 \pm 4.6$ & $200 \pm 26$ \\
$t \bar{t}$ & $218 \pm 41$ & $418 \pm 66$ & $429 \pm 77$ & $1,066 \pm 183$ \\
$W+$ jets & $1,773 \pm 199$ & $549 \pm 67$ & $149 \pm 42$ & $2,471 \pm 307$ \\
$Z+$ jets \& dibosons & $197 \pm 28$ & $73 \pm 21$ & $16 \pm 9.0$ & $286 \pm 57$ \\
Multijets & $133 \pm 12$ & $63 \pm 5.8$ & $21 \pm 2.0$ & $217 \pm 20$ \\
Total prediction & $2,443 \pm 292$ & $1,162 \pm 166$ & $635 \pm 134$ & $4,239 \pm 592$ \\
Data & $2,386 \pm 49$ & $1,161 \pm 34$ & $645 \pm 25$ & $4,192 \pm 65$ \\
$S: B$ & $1: 19$ & $1: 19$ & $1: 32$ & $1: 20$ \\
\hline
\end{tabular}


Table 7.4: Number of expected yields and observed data counts in "negative" samples after $b$-tagging.

\begin{tabular}{l||ccc|c} 
Source & 2 jets & 3 jets & 4 jets & All Channels \\
\hline \multirow{2}{*}{$t b$} & $52 \pm 7.6$ & $22 \pm 3.7$ & $6.5 \pm 1.6$ & $80 \pm 13$ \\
$t q b$ & $71 \pm 5.5$ & $36 \pm 4.2$ & $13 \pm 2.9$ & $120 \pm 13$ \\
$t b+t q b$ & $123 \pm 13$ & $58 \pm 7.9$ & $20 \pm 4.5$ & $200 \pm 25$ \\
$t \bar{t}$ & $217 \pm 42$ & $415 \pm 65$ & $433 \pm 78$ & $1,065 \pm 185$ \\
$W+$ jets & $1,789 \pm 191$ & $551 \pm 86$ & $135 \pm 40$ & $2,474 \pm 316$ \\
$Z+$ jets \& dibosons & $203 \pm 29$ & $68 \pm 19$ & $18 \pm 9.6$ & $289 \pm 56$ \\
Multijets & $144 \pm 13$ & $67 \pm 6.2$ & $22 \pm 2.1$ & $233 \pm 21$ \\
Total prediction & $2,475 \pm 287$ & $1,157 \pm 183$ & $628 \pm 133$ & $4,261 \pm 603$ \\
Data & $2,495 \pm 50$ & $1,146 \pm 34$ & $638 \pm 25$ & $4,279 \pm 65$ \\
$S: B$ & $1: 19$ & $1: 19$ & $1: 31$ & $1: 20$ \\
\hline
\end{tabular}




\subsection{Signal acceptance}

The signal acceptance is defined as:

$$
\mathcal{A}=\frac{\mathcal{B}}{\mathcal{N}_{\text {initial }}} \sum_{\mathcal{N}_{\text {selected }}} \varepsilon_{\text {corrections }}
$$

where $\mathcal{B}$ is the branching fraction for the $\mathrm{MC}$ sample, $\mathcal{N}_{\text {initial }}$ is the initial number of events in each MC sample, $\mathcal{N}_{\text {selected }}$ is the number of $\mathrm{MC}$ events remaining after selection, and $\varepsilon_{\text {corrections }}$ are efficiency correction factors to account for differences between data and MC from particle ID, vertex confirmation, taggability and $b$-tagging.

Table 7.5 shows the percentage of each single top quark signal that remain after selection in each channel before and after $b$-tagged jets have been required. The entries in this table are calculated using the yields shown in Section 7.1 and the following equation:

$$
\mathcal{A}=\frac{\text { Yield }}{\sigma \times \mathcal{L}}
$$

in which $\sigma$ is the predicted cross section (for $t b$ or $t q b$ ) and $\mathcal{L}$ is the integrated luminosity for each sample. 
Table 7.5: Combined (Electron+Muon, Run IIa+Run IIb) signal acceptances.

\begin{tabular}{l||ccc|c} 
& 2 jets & 3 jets & 4 jets & All Channels \\
\hline PreTag & & & & \\
$t b$ & $3.0 \%$ & $1.3 \%$ & $0.39 \%$ & $4.7 \%$ \\
$t q b$ & $2.6 \%$ & $1.2 \%$ & $0.41 \%$ & $4.3 \%$ \\
$t b+t q b$ & $2.8 \%$ & $1.2 \%$ & $0.40 \%$ & $4.4 \%$ \\
1,2 Tags & & & & \\
$t b$ & $1.9 \%$ & $0.78 \%$ & $0.23 \%$ & $2.9 \%$ \\
$t q b$ & $1.2 \%$ & $0.60 \%$ & $0.21 \%$ & $2.0 \%$ \\
$t b+t q b$ & $1.4 \%$ & $0.65 \%$ & $0.22 \%$ & $2.3 \%$ \\
\hline
\end{tabular}

The final combined signal acceptances are:

$$
\begin{aligned}
\mathrm{s}-\text { channel } t b & =(2.9 \pm 0.4) \% \\
\mathrm{t}-\text { channel } t q b & =(2.0 \pm 0.3) \% \\
\mathrm{~s}+\mathrm{t} \text { channels } t b+t q b & =(2.3 \pm 0.3) \%
\end{aligned}
$$

\subsection{Data and Monte Carlo comparison}

We examine various important kinematic variables to make sure the Monte Carlo simulated samples model the data. To judge whether a variable is well modeled or not, the variable distribution for data is compared with the sum of the signal and 
backgrounds. We normalize each sample in the model to the integrated luminosity of data (5.4 ifb), and overlay their sum to the data.

The data-MC comparison plots after the selection cuts in Section 5.2 for both pretagged and tagged samples are shown in Appendix ??.

The data-MC comparison plots after splitting by the lepton charge are shown in Appendix A. The data-MC comparison plots for the combined BNN discriminants after splitting are shown in Appendix C.

Good agreement is seen between the prediction and data in all channels. Selected plots after splitting by the lepton charge are shown below.
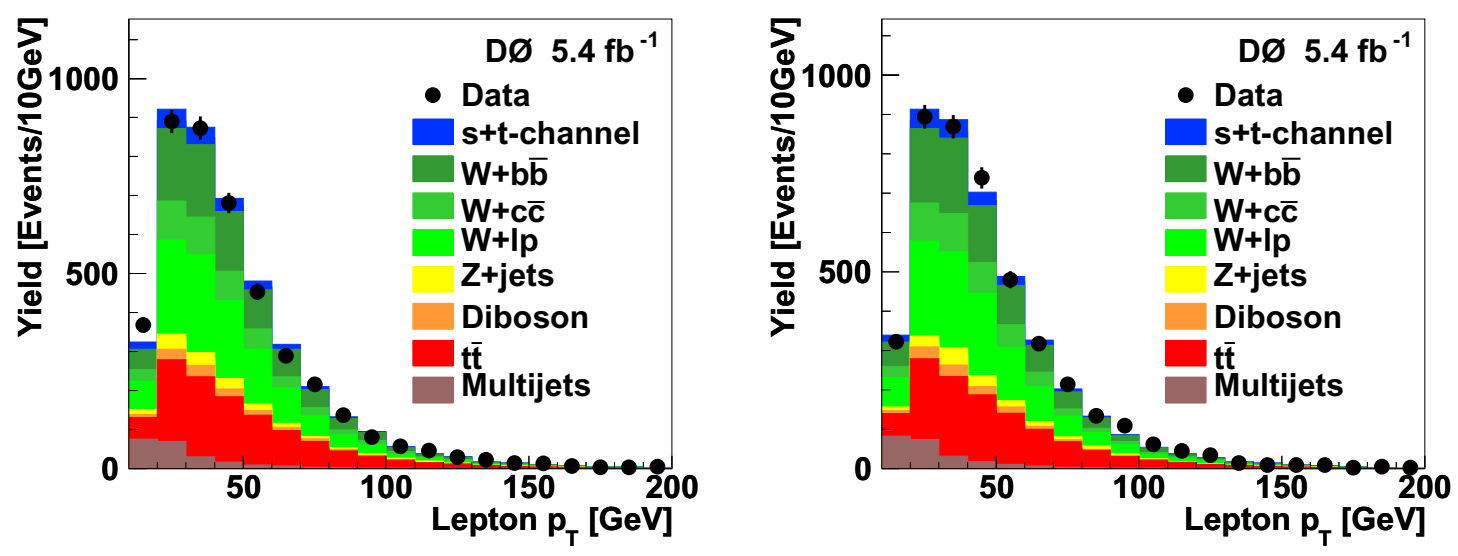

Figure 7.2: The transverse momentum of the lepton in the electron+muon channel for positive (left) and negative (right) samples. 

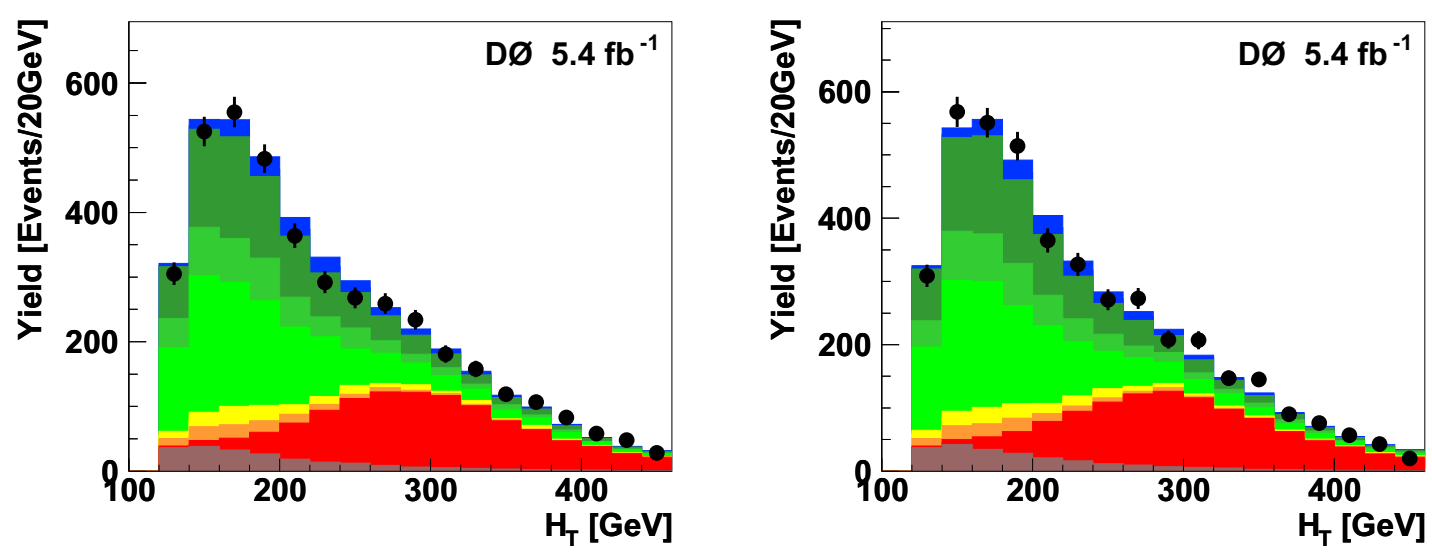

Figure 7.3: The HT in the electron+muon channel for positive (left) and negative (right) samples.
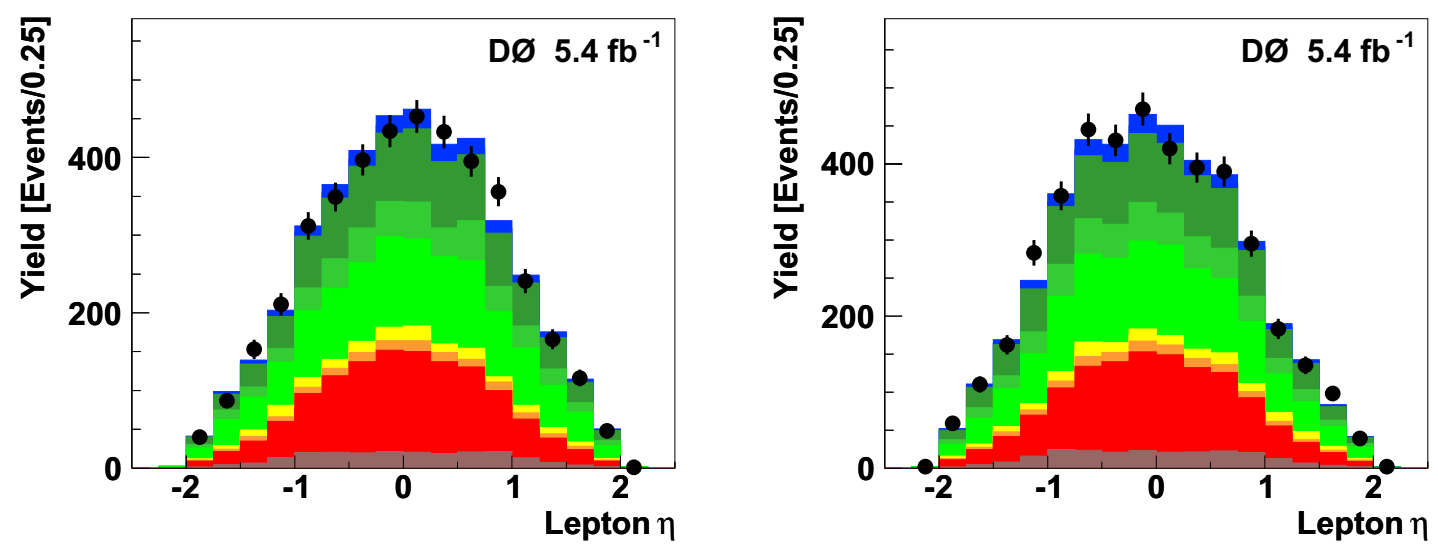

Figure 7.4: The LeptonEta in the electron+muon channel for positive (left) and negative (right) samples. 

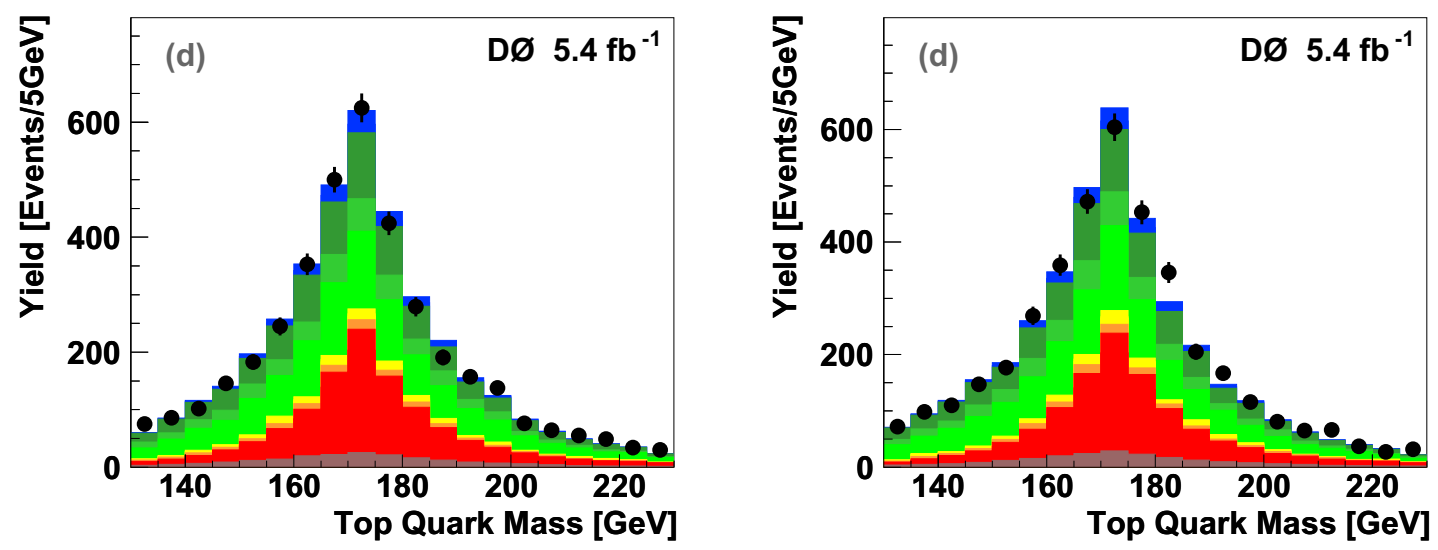

Figure 7.5: Reconstructed top mass distribution in the electron+muon channel for positive (left) and negative (right) samples.
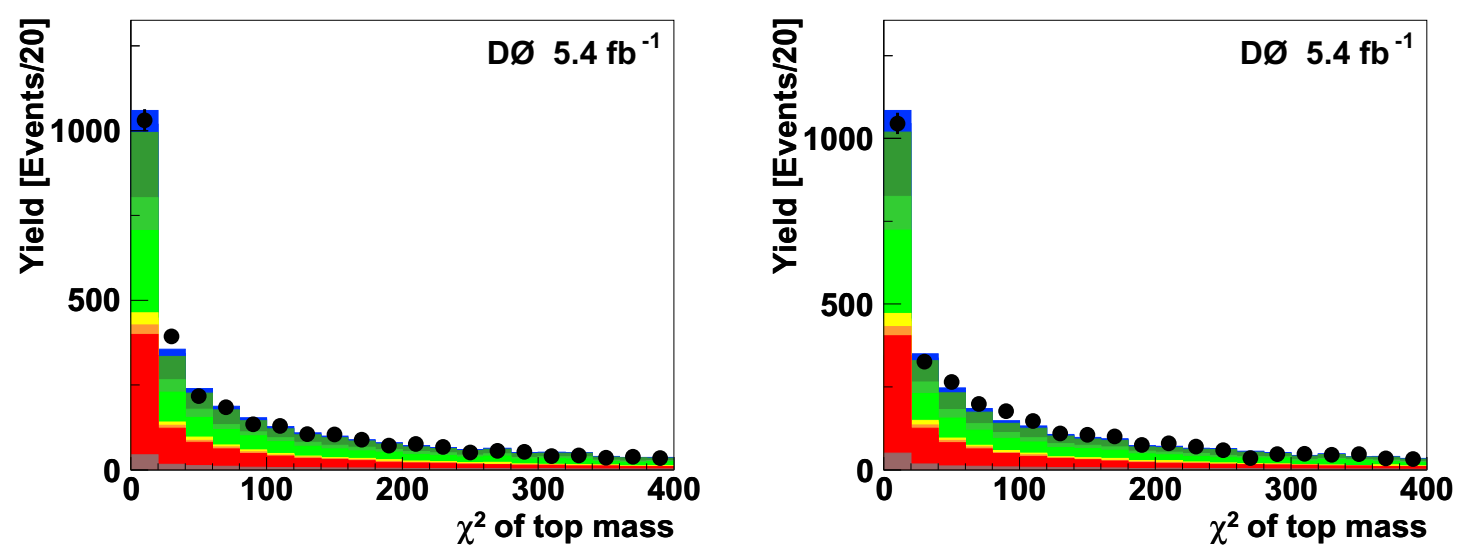

Figure 7.6: Top mass minimum Chi squared distribution in the electron+muon channel for positive (left) and negative (right) samples. TopMassMinChiSqr gives the minimum value of the differences between constructed top mass and theory top mass for all possible reconstructed top quarks. 

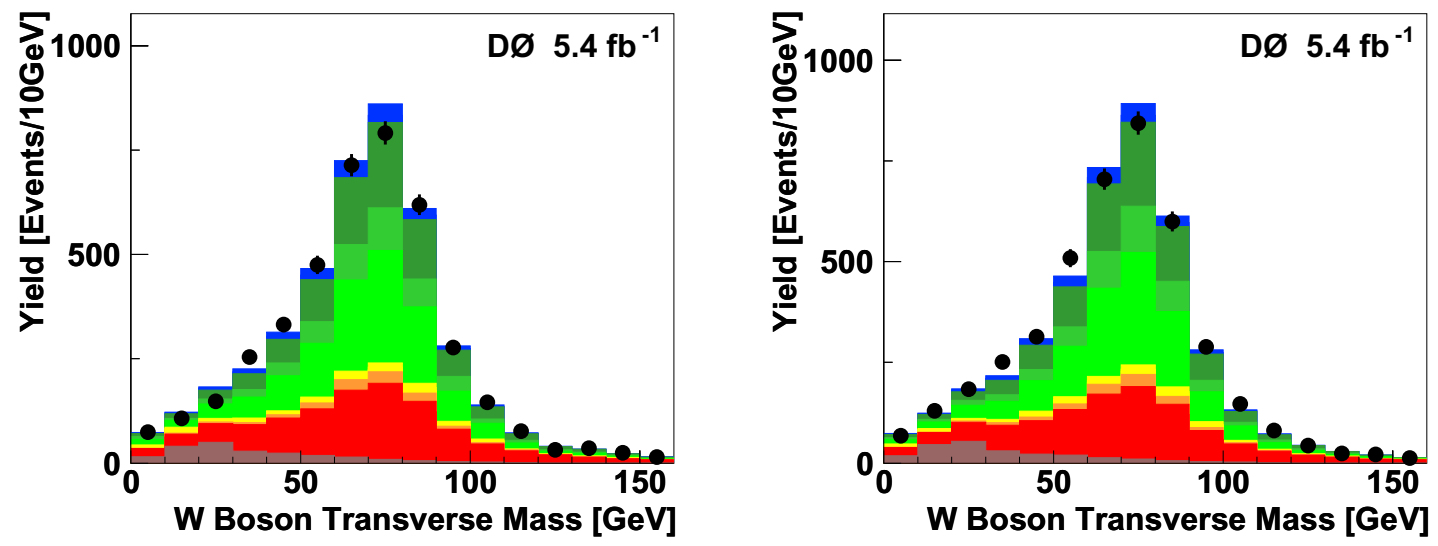

Figure 7.7: The W Transverse mass distribution in the electron+muon channel for positive (left) and negative (right) samples. 


\section{Chapter 8}

\section{Multivariate Analysis}

Given the increasing complexity of modern HEP experiments, multivariate analysis (MVA) techniques have been applied to extract the maximum information content out of the data. Multivariate classification is one of the techniques used for "data mining", which is automatic or semi-automatic analysis of large amount of data in order to extract previously unknown interesting patterns. MVA involves analysis of multiple statistical variables at a time.

Three different MVA techniques are used in the single top analysis: (i) Bayesian neural networks (BNN), (ii) boosted decision trees (BDT), and (iii) neuroevolution of augmented topologies (NEAT). A combined new BNN discriminant is formed by further combining those three methods.

A big advantage of multivariate techniques compared with a conventional cut 
based analysis is that events which fail an individual selection criterion will continue to be considered by the algorithm. In a multivariate technique, the separation power of the many discriminating variables $\mathbf{x}$ is combined into a single discriminant $D(\mathbf{x})$. This way we do not cut out signal events, while obtaining an improved separation power beyond that of any of the single variables alone. This combined single variable corresponds to a one-to-one function of the probability $\operatorname{Pr}(S \mid \mathbf{x})$ that an event characterized by the variables $\mathbf{x}$ belongs to the signal class S. For each MVA, a background event is more likely to give an output in the low-discriminant region $(D(\mathbf{x}) \approx 0)$, while a signal event will tend to output to the high-discriminant region $(D(\mathbf{x}) \approx 1)$, thus separating signal from the backgrounds. The cross section and other info can be derived from the $D(\mathbf{x})$ distributions observed in data using Bayesian calculations which will be discussed in Chapter 9.

Figure 8.1 shows how each of the MVA's is applied in the flow chart of the single top analysis.

\subsection{Split samples}

The signal and background samples are divided into three independent subsets, which are used by the three MVA's.

- the first subset ("training") contains one fourth of the samples and is used to created the multivariate filters for (i.e. train) BDT, BNN and NEAT; 


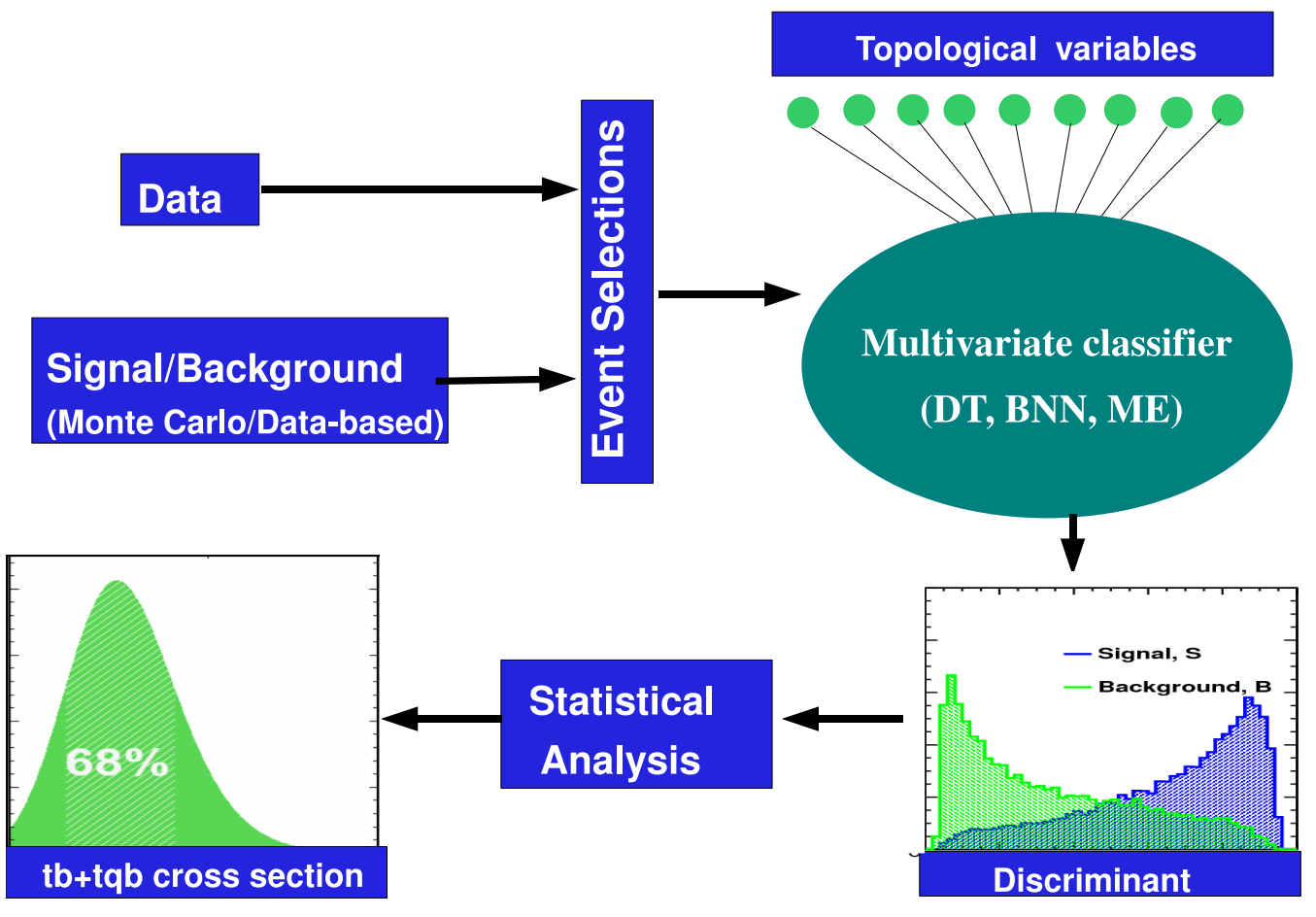

Figure 8.1: Flowcharts of the MVA analyses. 
- the second subset ("testing") also contains one fourth of the samples and is used to created the multivariate filter (i.e. train) the combination BNN of the three different multivariate analyses (BDT, BNN and NEAT) used in the single top analysis;

- the last subset ("yield") contains the remaining half of the samples and is used to verify the BNNs convergence, do the final measurements with all the MVAs (BDT, BNN, NEAT, and combination BNN) and make all the plots.

The original MC samples are split by putting the first event in the testing, the second event in the training and the 3rd and 4th event in the yield sample, and repeating the process until the end of the sample was reached. All three resulting samples are normalized back to the full dataset: testing/training weights multiplied by 4.0 and yield weights multiplied by 2.0 , where the actual factors are calculated as yield in the original over yield in the subsample.

\subsection{Output transformation}

The MVA output is a number between 0 and 1, with background-like (signal-like) events populating mostly in the low (high) discriminant region. In the high discriminant region there may be some bins in which there are some signal but no background events. To avoid this, we apply a monotonic transformation to the discriminant that 
ensures a minimum number of background events in each bin. For details see Appendix D of $D \varnothing$ Note 5811 [58]. All the discriminant plots shown in this chapter are after applying this binning transformation, unless otherwise noted. 


\subsection{Key for plots}

For each MVA output plot, the signal and background distributions use the color scheme illustrated in Figure 8.2.

\begin{tabular}{|c|}
\hline 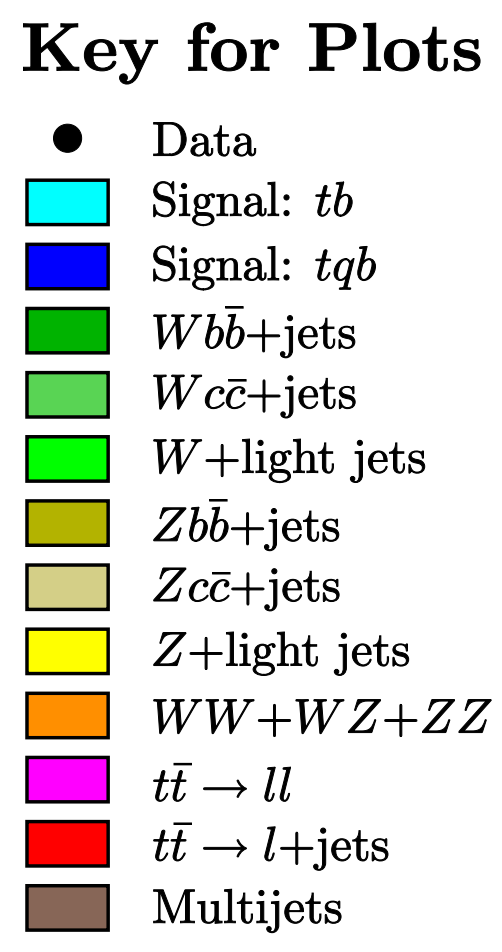 \\
\hline
\end{tabular}

Figure 8.2: Illustration of the color scheme used in plots of signals and backgrounds in the single top analyses. 


\subsection{Boosted Decision Trees (BDT)}

Decision trees (DT) originated in the fields of data mining and pattern recognition. Several methods were developed to improve the DT classification power by creating an ensemble (forest) of decision trees. One of these extensions is boosting decision trees $(\mathrm{BDT})$.

In a decision tree, the internal nodes contains various tests, and terminal nodes are called leaves. Each leaf has an assigned decision tree output value. An event will start from the root node, follow a unique path through the decision tree, and end up at a leaf and the classification of the event is the decision tree output value of this leaf. All nodes continue to be split until they become leaves.

Figure 8.3 shows an example of a decision tree.

A powerful technique to improve the performance of any weak classifier (defined as any classifier that does a little better than random guessing) is boosting [59]. The basic principle of boosting is to create a tree, calculate an associated error function then regenerate a tree with a smaller error function by re-weighting the misclassified events. The boosting algorithm used in D $\varnothing$ 's single top quark search is adaptive boosting (AdaBoost) [59].

In this analysis we find that boosting typically improves the performance by $20 \%$. The increase in performance saturates in the region of 25 boosting cycles, therefore 30 boosts were used in this analysis. The full set of parameters used in this analysis 


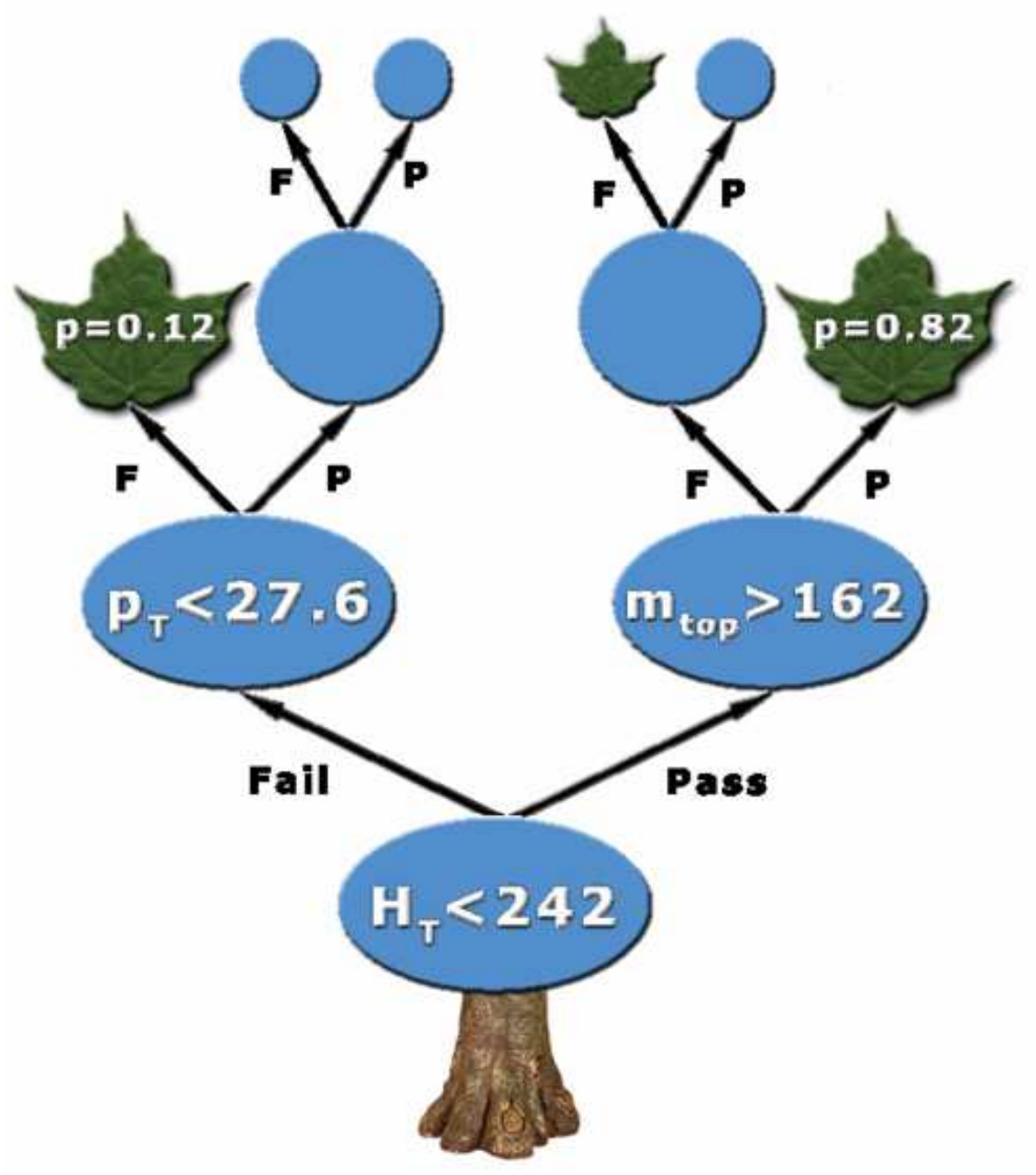

Figure 8.3: An example decision tree. Nodes with associated splitting test are shown as blue circles and terminal nodes with purity output are shown as green leaves. An event (observation) defined by variables $x_{i}$ of which $H_{T}<242$, and $m_{t o p}>162$ will return $D\left(x_{i}\right)=0.82$. An event with variables $x_{j}$ of which $H_{T} \geq 242$ and $p_{T} \geq 27.6$ will result a decision tree output value 0.12 . 
Table 8.1: BDT discriminant used in the current analysis.

\begin{tabular}{lr}
\hline \hline parameter & value \\
\hline Number of Boosting Cycles: & 30 \\
Ada Boost Parameter: & 0.20 \\
Minimal Leaf Size: & 100 \\
Impurity Measure: & Gini \\
Boosting Purity Limit: & 0.5 \\
Number of Bags: & 1 \\
Bagging Fraction: & 0.75 \\
Random Forest Fraction: & 1.0 \\
\hline \hline
\end{tabular}

is as shown in Table 8.1.

Figs. 8.4, 8.5, and 8.6 show the distributions for BDT, for the 6 analysis channels and the combination, for $s+t$-, $s$ - and $t$ - channels, respectively. 
BDT OUTPUT FOR s+t CHANNEL

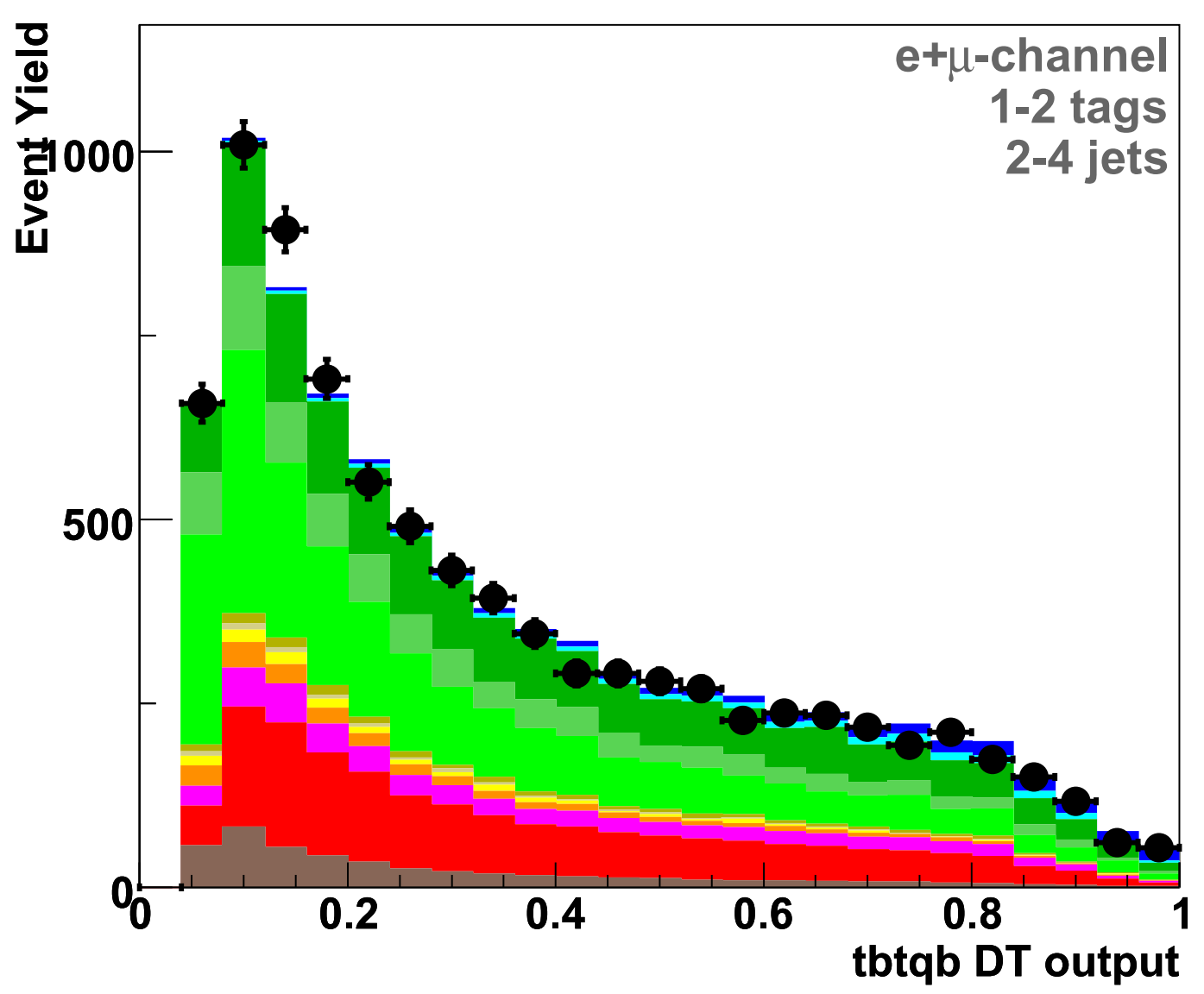

Figure 8.4: BDT output for $s+t$ channel for the six analysis channels combined. 
BDT OUTPUT FOR s CHANNEL

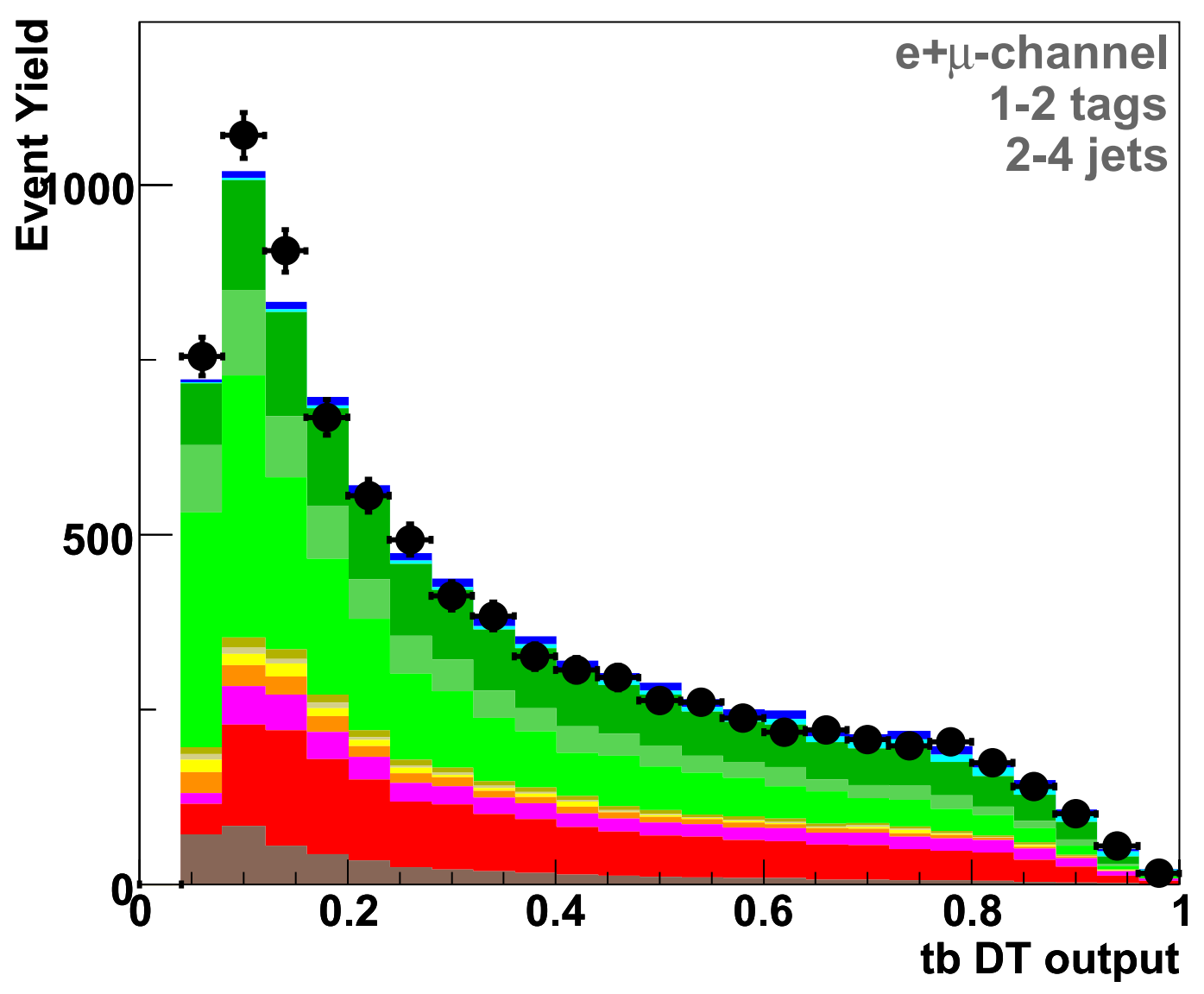

Figure 8.5: BDT output for $s$-channel for the six analysis channels combined. 
BDT OUTPUT FOR t CHANNEL

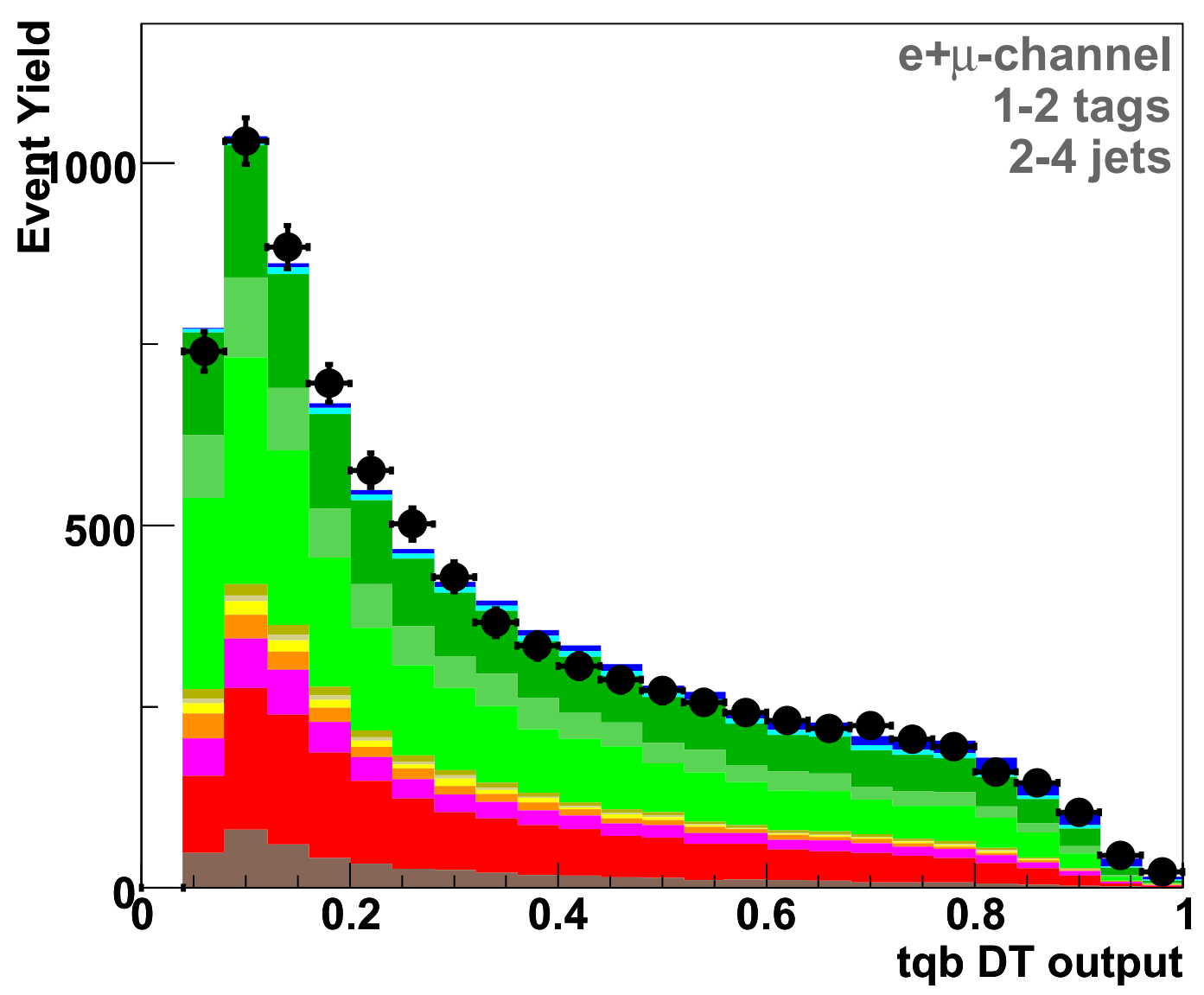

Figure 8.6: BDT output for $t$-channel for the six analysis channels combined.

\subsection{Bayesian Neural Networks (BNN)}

Artificial "neural networks" (NN) are widely used as flexible models for classification.

Bayesian methods can improve the power of these models, so that they can be safely 
exploited when training data is limited. Figure 8.7 shows an example of a simple Neural Network.

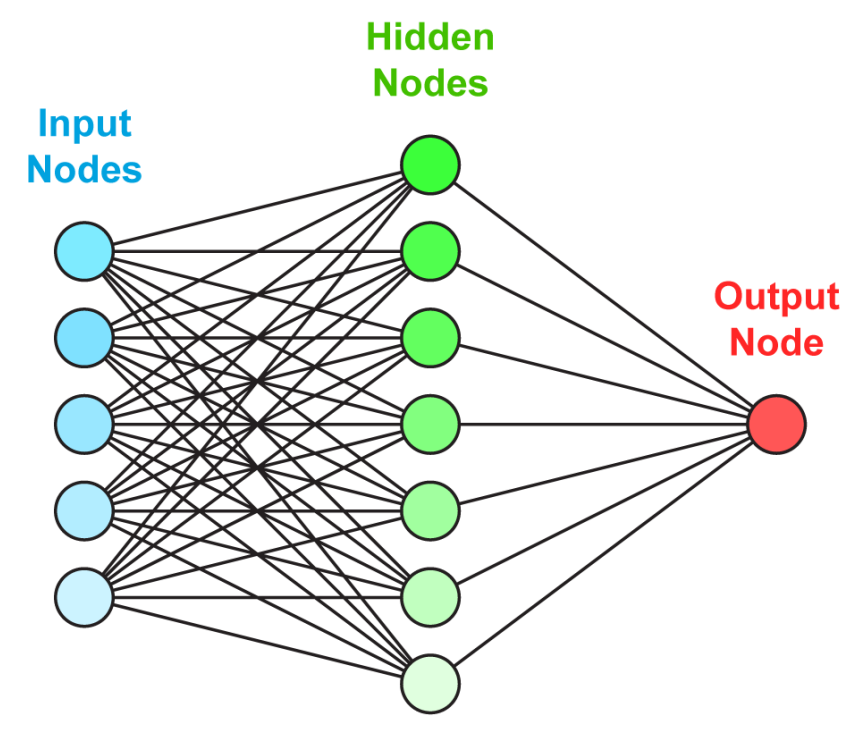

Figure 8.7: An illustration of a simple Neural Network.

The BNN analysis proceeds as follows.

1. The BNN analysis starts from the lepton and jet 4-vectors variables with good discrimination between signal and background for 6 channels.

2. The training of the networks is performed and the discriminant $D(\mathbf{x})$ is built. We then check that the performance of the BNN is adequate.

3. Compute the expected and observed posterior densities of the single top cross section for each of the 6 analysis channels and its combinations using binned 
likelihoods formed from the BNN output distributions of the 6 analysis channels, and determine the cross sections.

For each of the 6 analyses channels we built a training set $T$ by combining 10,000 signal and 10,000 background events, which were sampled proportionally to their weights from the signal and background in the MC training samples. From the training set $T$ we constructed a posterior density $p(w \mid T)$ over the network parameter space. A sample of $K=100$ networks pertaining to the last 100 of 300 epochs was drawn from the posterior density using a Markov Chain Monte Carlo (MCMC) technique $[60,61]$ and used to approximate the discriminant, D.

Figures $8.8,8.9$, and 8.10 show the BNN output distributions for the 6 analysis channels and the combination, for s+t-, s- and t- channels, respectively. 
BNN OUTPUT FOR $s+t$ CHANNEL

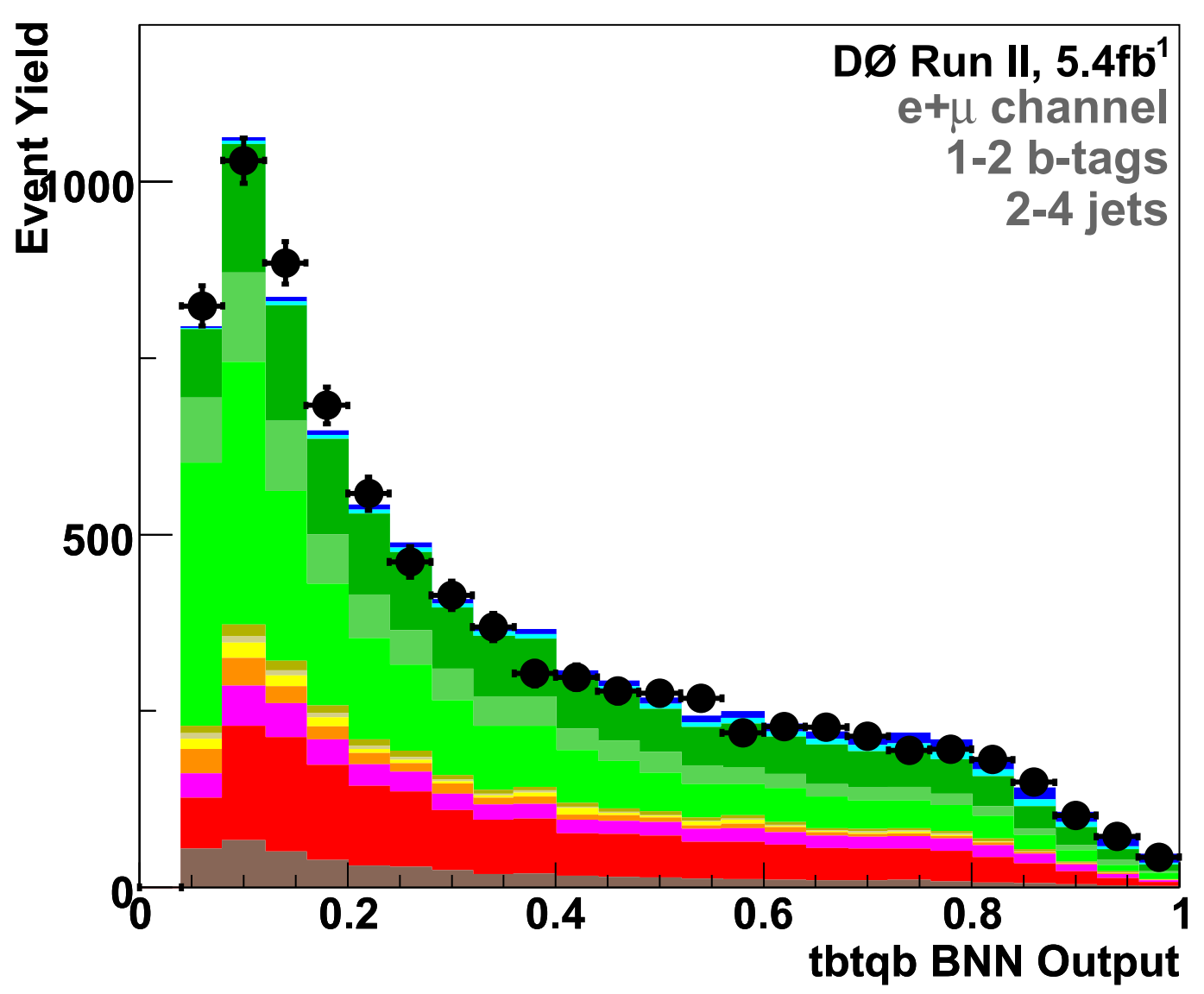

Figure 8.8: BNN output for $s+t$ channel for the six analysis channels combined. 
BNN OUTPUT FOR s CHANNEL

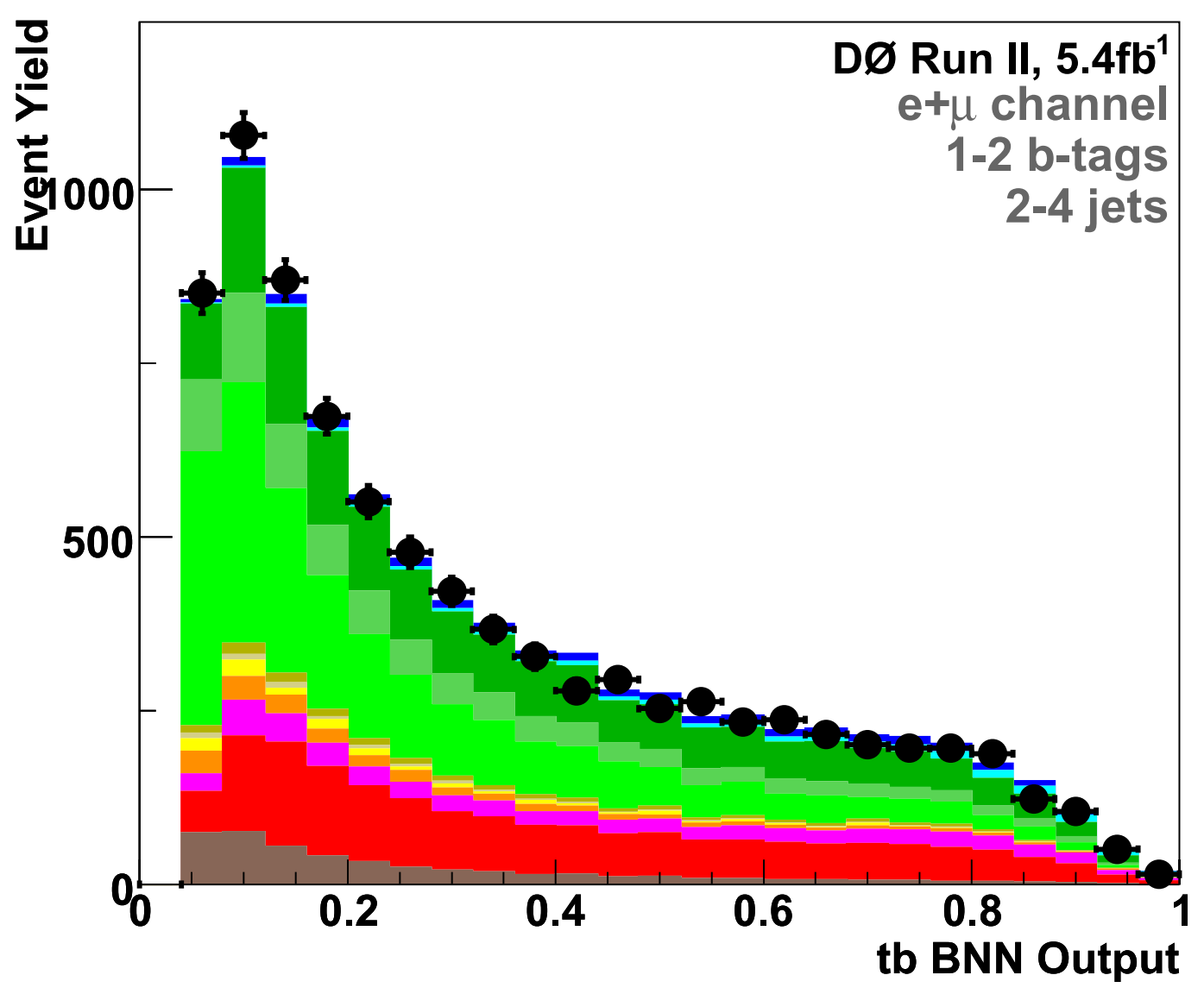

Figure 8.9: BNN output for $s$-channel for the six analysis channels combined. 


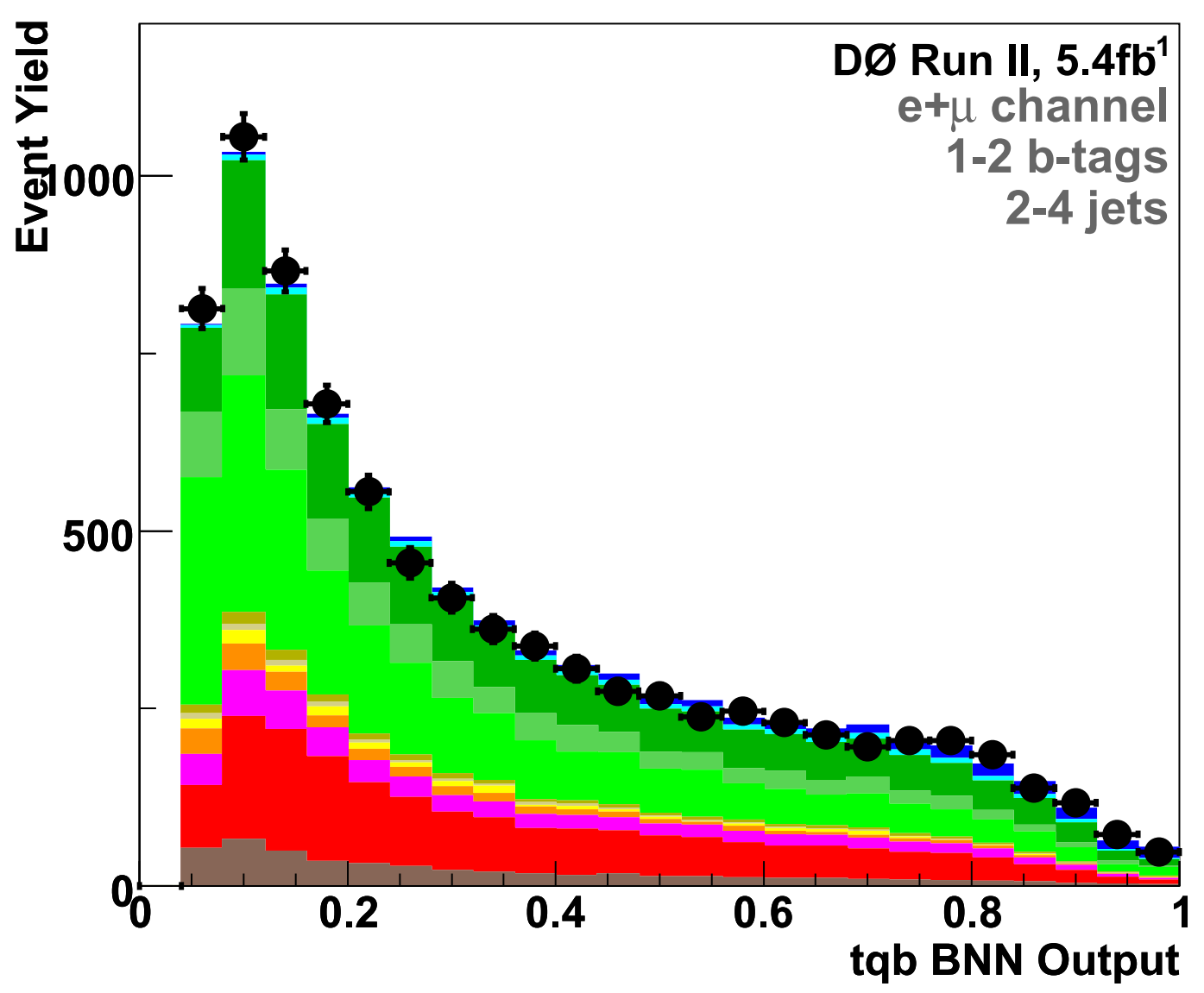

Figure 8.10: BNN output for $t$-channel for the six analysis channels combined.

\subsection{Neuroevolution of augmented topologies (NEAT)}

NeuroEvolution of Augmenting Topologies (NEAT) is a method for evolving artificial neural networks with a genetic algorithm. It is most effective to start evolution 
with small, simple networks and allow them to become increasingly complex over generations, just as organisms in nature increased in complexity since the first cell.

The training for NEAT is based on a genetic algorithm which evolves a population of possible discriminators. The decriminators of NEAT are defined by neural networks (NN) that are similar to those used in BNN. An initial population of NNs is created with zero hidden neurons and randomly assigned weight connections. This population is then evolved by a series of operations that can be classify as mutations, crossovers and selection. Mutations change the weight of existing connections or add new hidden nodes to the network. Crossovers create a new NN by combining two different parent NNs, allowing the combination of those features that potentially can create better discriminators. After these operations, NNs are selected to be part of the next generation depending on the "fitness" of each discriminator.

Figs. 8.11, 8.12, and 8.13 show the NEAT output distributions for the 6 analysis channels and the combination, for s+t-, s- and t- channels, respectively [62]. 


\section{NEAT OUTPUT FOR $\mathrm{s}+\mathrm{t}$ CHANNEL}

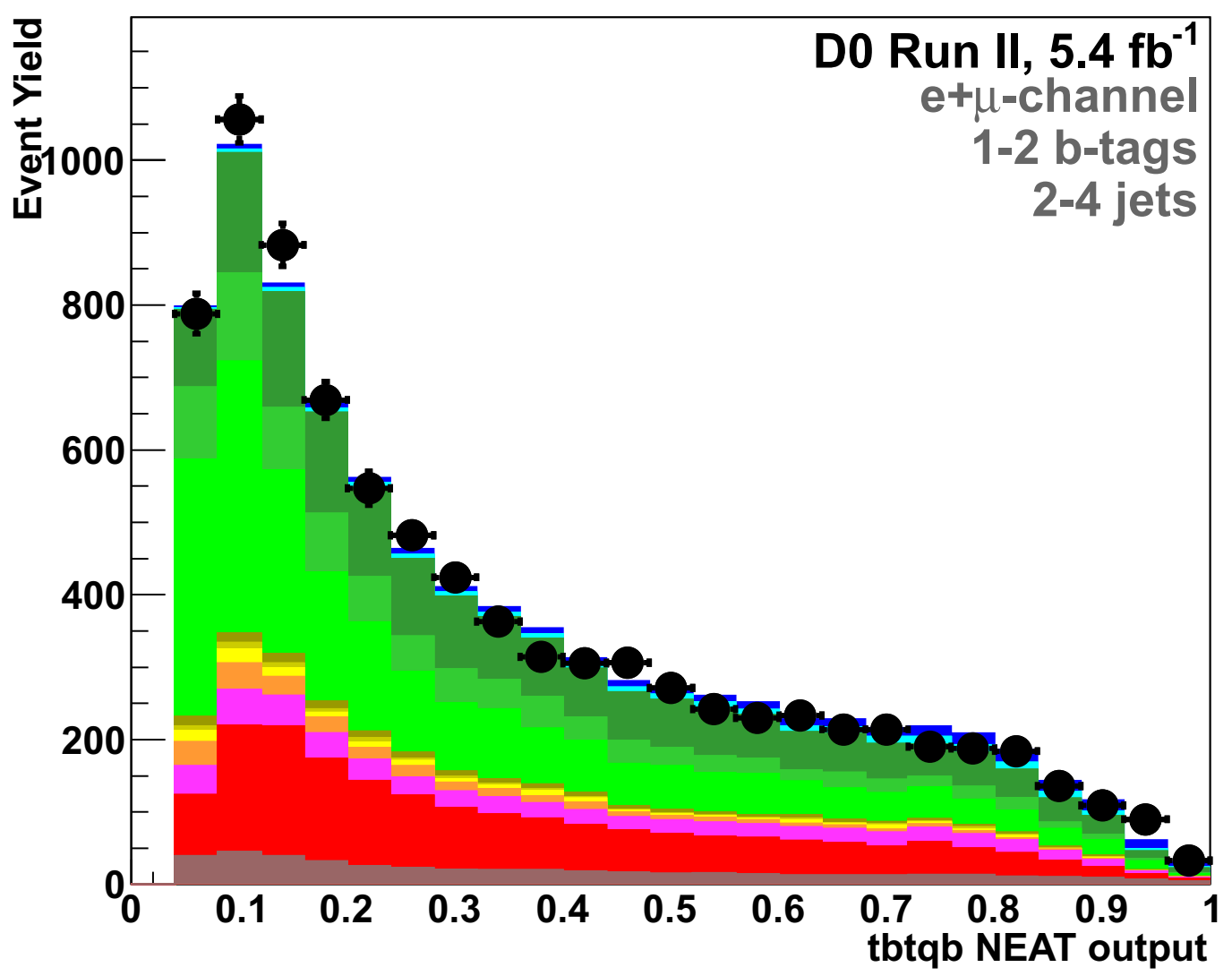

Figure 8.11: NEAT output for $s+t$ channel for the six analysis channels combined. 
NEAT OUTPUT FOR s CHANNEL

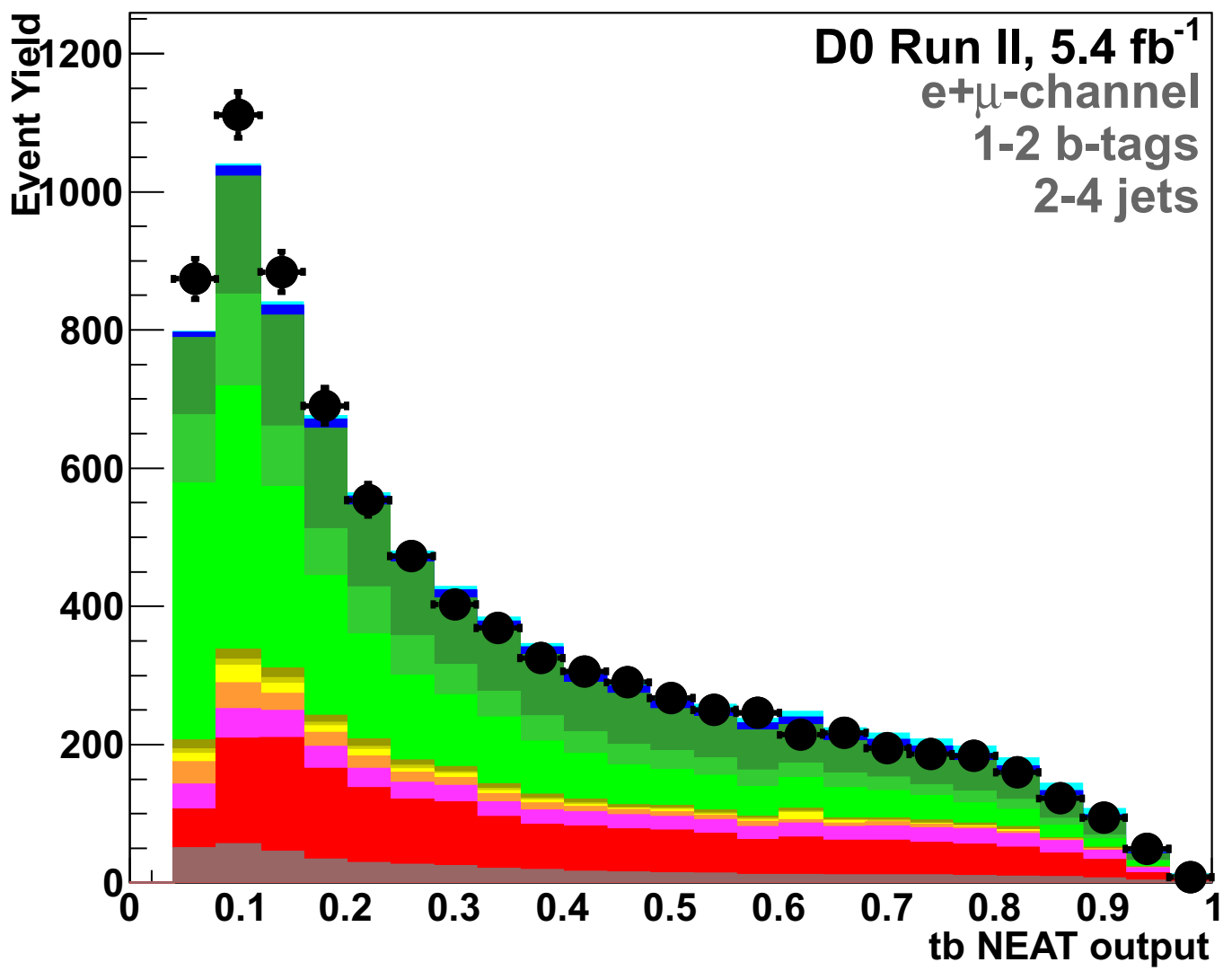

Figure 8.12: NEAT output for $s$-channel for the six analysis channels combined. 


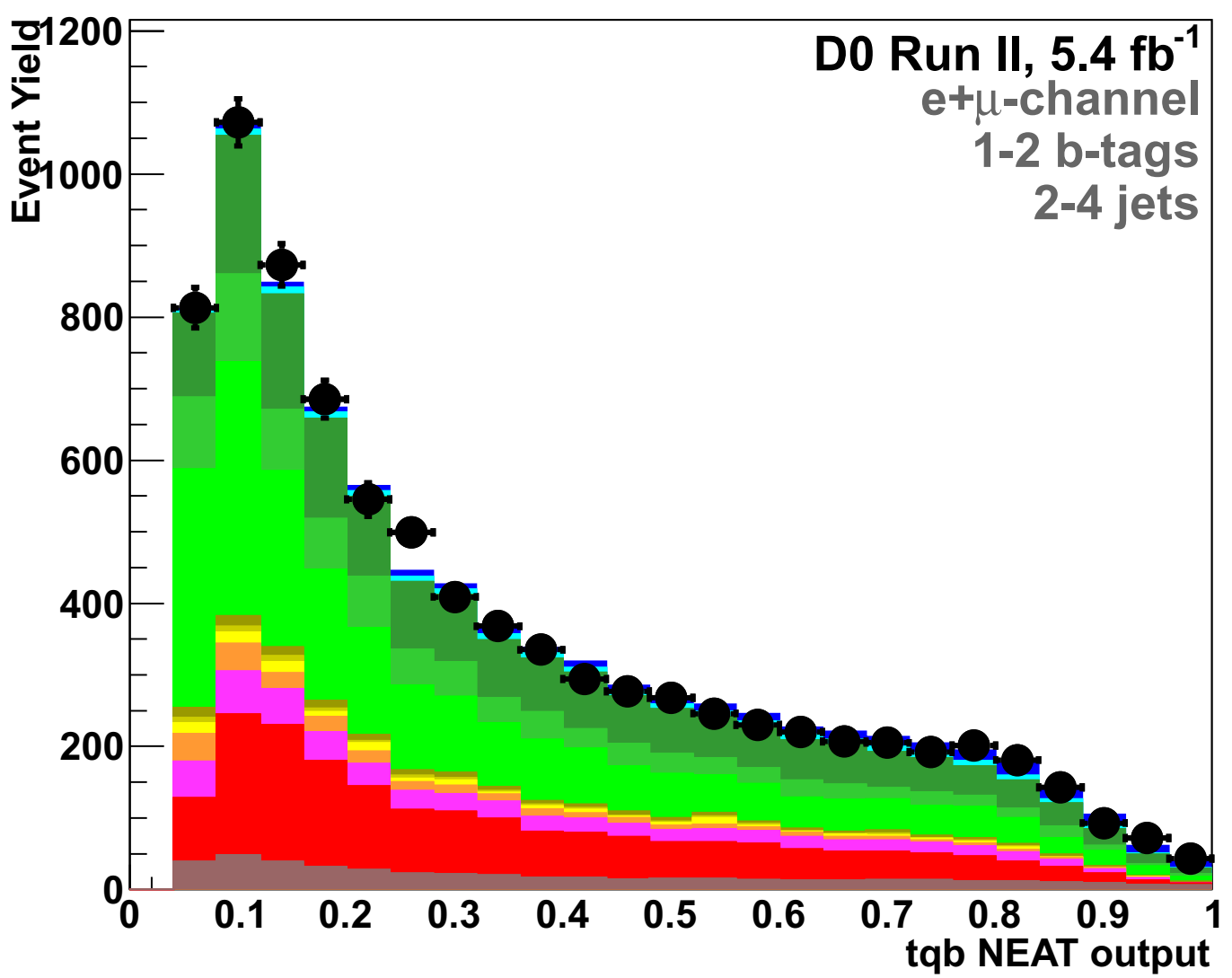

Figure 8.13: NEAT output for $t$-channel for the six analysis channels combined. 


\subsection{A combined BNN using BDT, BNN and NEAT discriminants}

Ensemble tests with datasets containing contributions only from backgrounds and SM signals, shows a $70 \%$ correlation among the outputs of the individual MVA methods above. Thus, to achieve the maximum sensitivity, a second level of BNN is used to construct a combined discriminant for each channel, for $t b$, $t q b$, and $t b+t q b$ events, using BDT, BNN and NEAT discriminants as the inputs.

In this combination BNN, we have two sets of trainings. The combined $t b$ and tqb discriminants take as inputs the three discriminant outputs of BDT, BNN, and NEAT, and they are trained by assuming $t b$ or $t q b$ as signals, respectively. The combined $t b+t q b$ discriminant takes inputs from the six discriminant outputs of BDT, BNN, and NEAT that are trained separately for the $t b$ and the $t q b$ signal. The training for the combined $t b+t q b$ BNN treats the combined $t b+t q b$ contribution as signal with production rates ratio predicted by SM. In both trainings, we followed the same procedure as in the non-combination BNN training, described in Section 8.5. The CP violation analysis uses this combined BNN output as the basis.

Figs. 8.14, 8.15, and 8.16 show the NEAT output distributions for the 6 analysis channels and the combination, for s+t-, s- and t- channels, respectively. 
BNN Combination OUTPUT FOR s+t CHANNEL

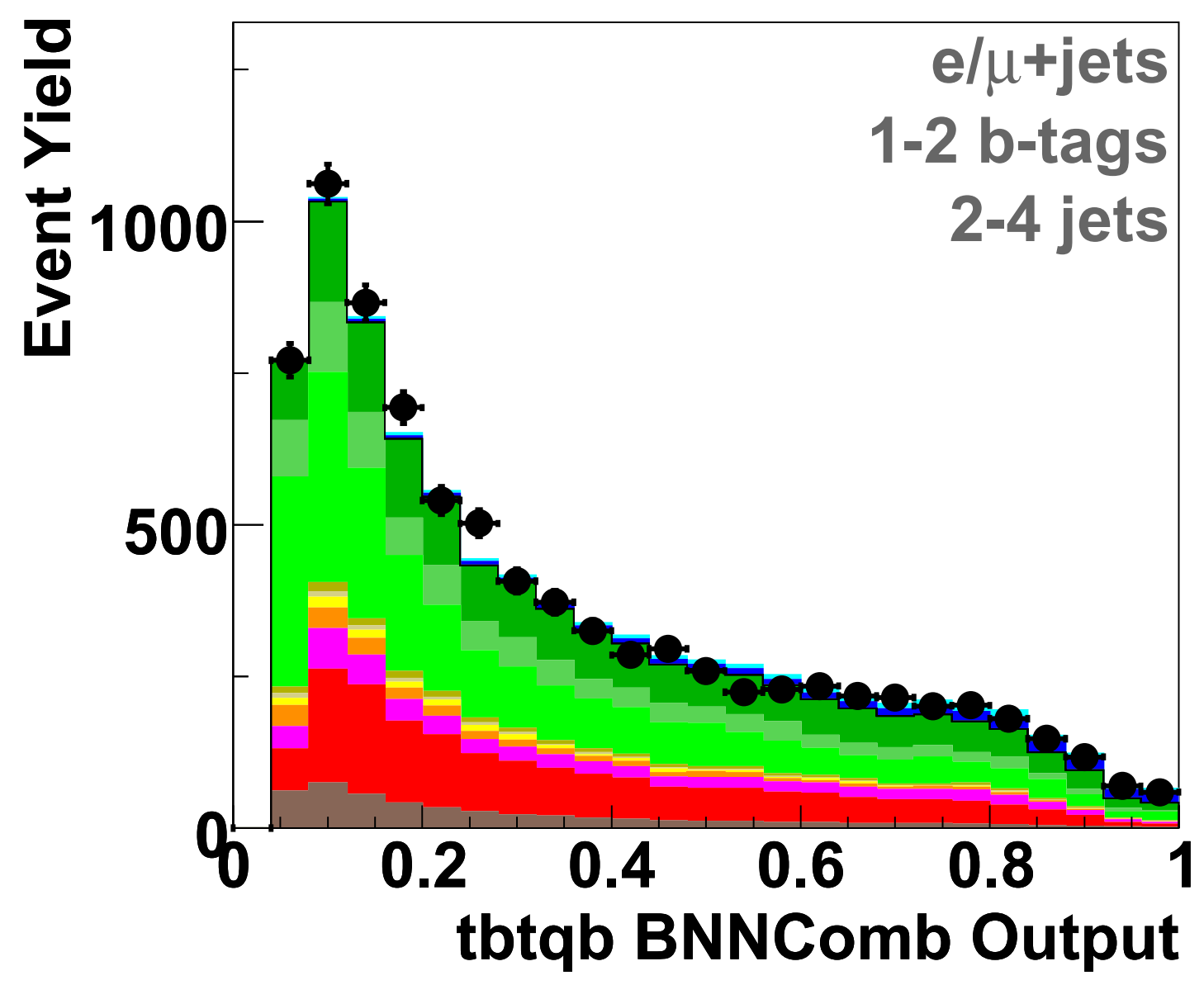

Figure 8.14: Combined BNN output for $s+t$ channel for the six analysis channels combined. 
BNN Combination OUTPUT FOR s CHANNEL

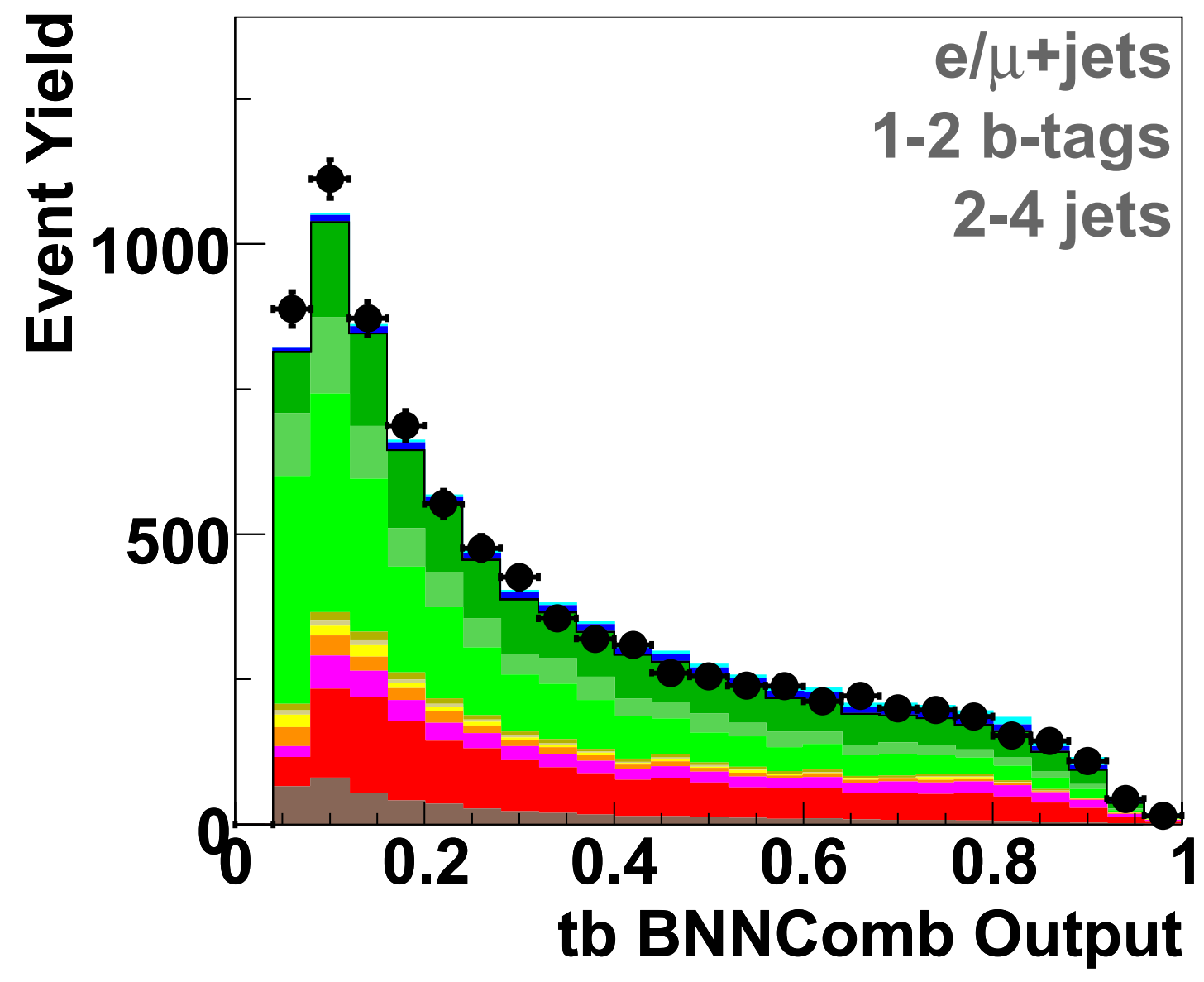

Figure 8.15: Combined BNN output for $s$ channel for the six analysis channels combined. 
BNN Combination OUTPUT FOR t CHANNEL

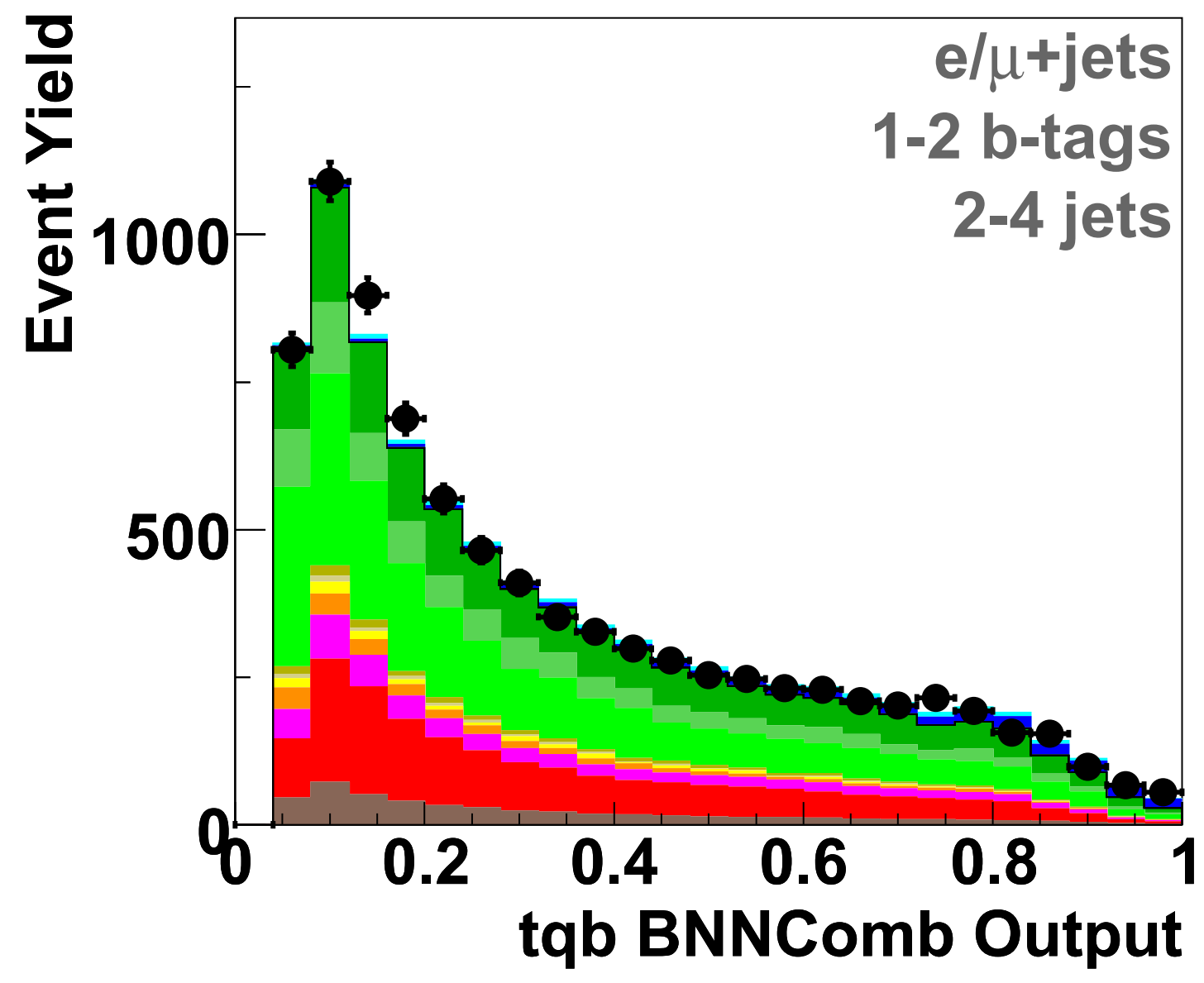

Figure 8.16: Combined BNN output for $s+t$ channel for the six analysis channels combined.

Figures C.1 to C.6 show the combined discriminants distributions for positive and negative samples separately after splitting by lepton charge. 

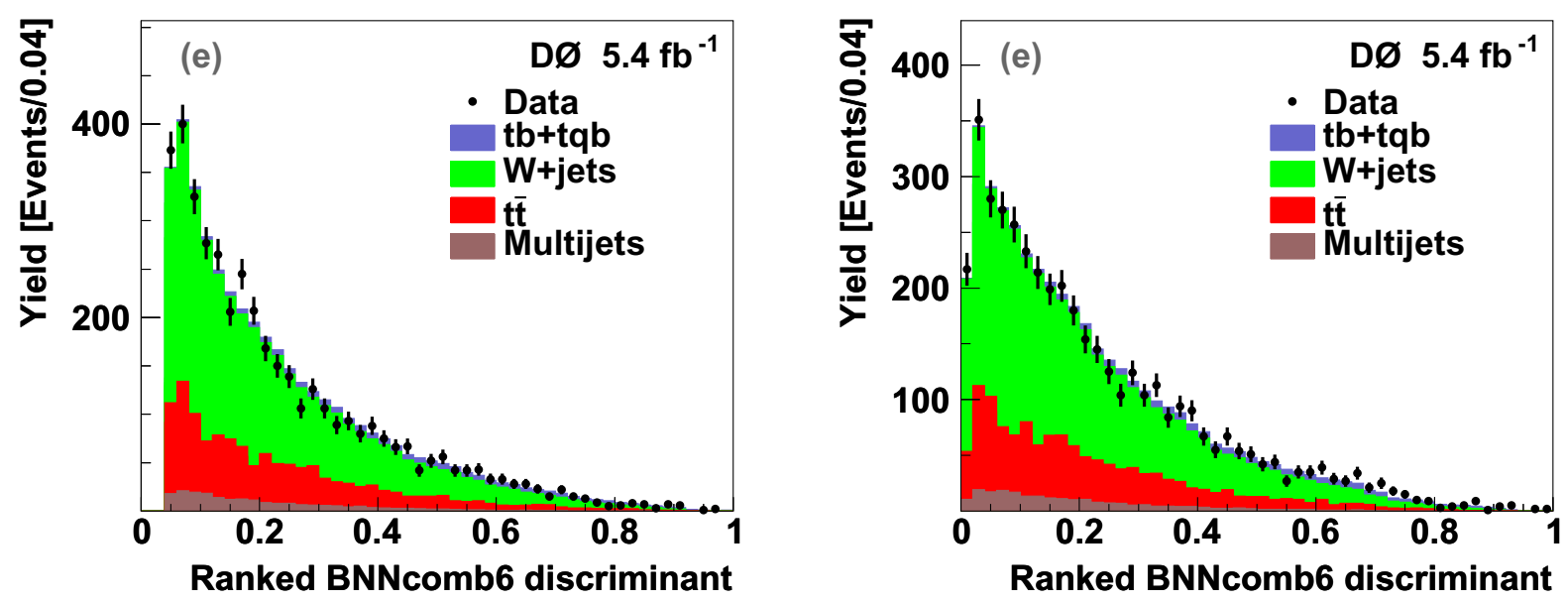

Figure 8.17: The $s+t$ discriminant plots in the electron + muon channel for positive (left) and negative (right) events.
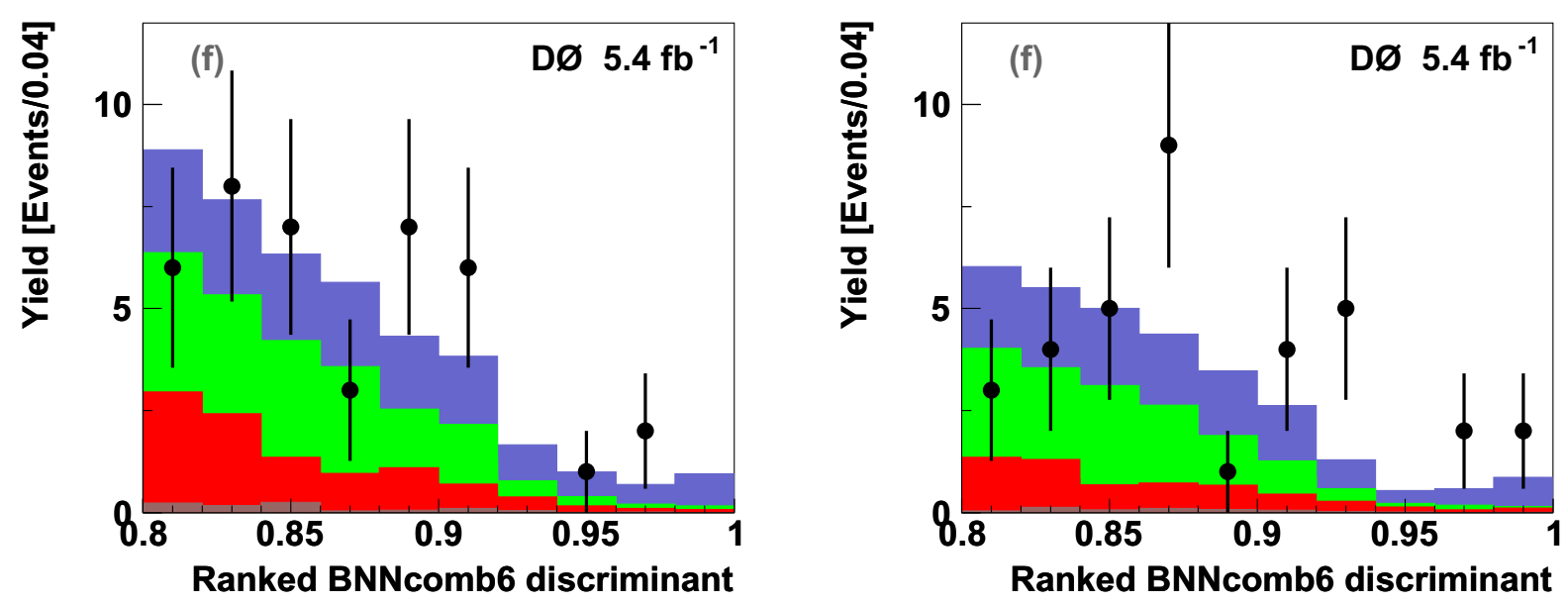

Figure 8.18: The BNNcomb6SortZoom plots in the electron+muon channel for positive (left) and negative (right) events. 

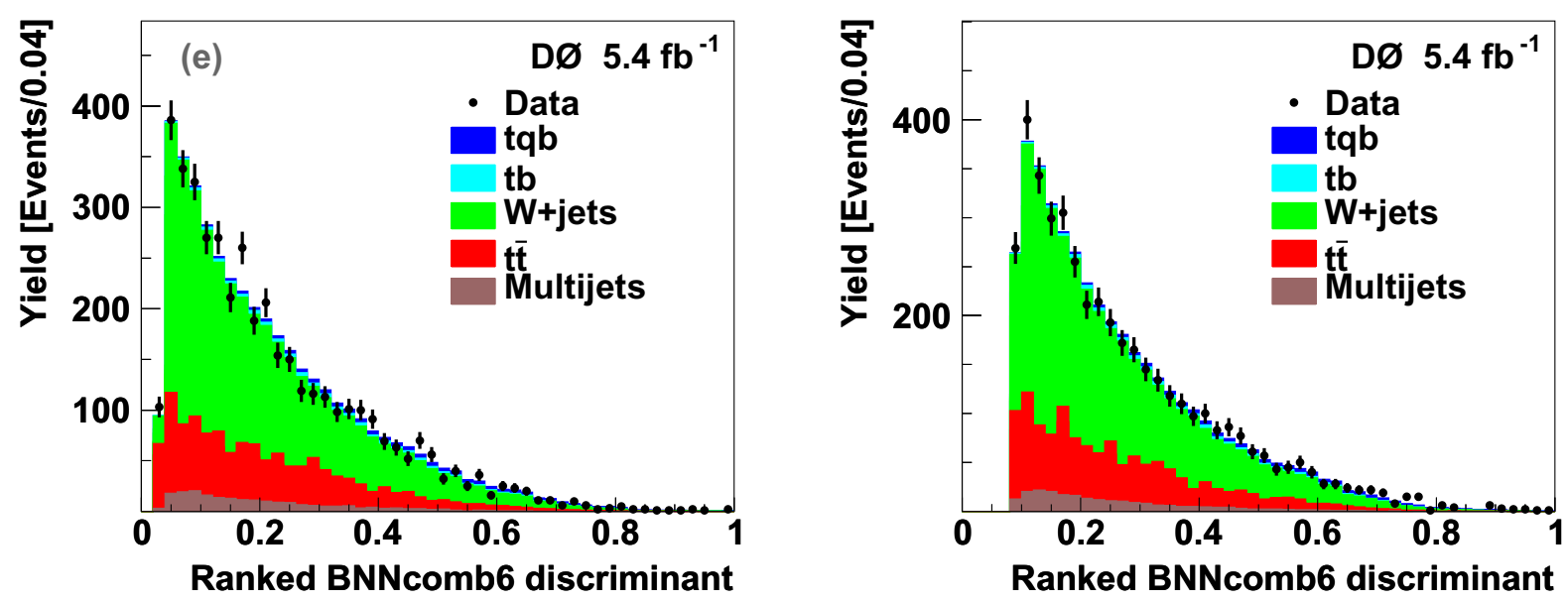

Figure 8.19: The $t$ discriminant plots in the electron+muon channel for positive (left) and negative (right) events.
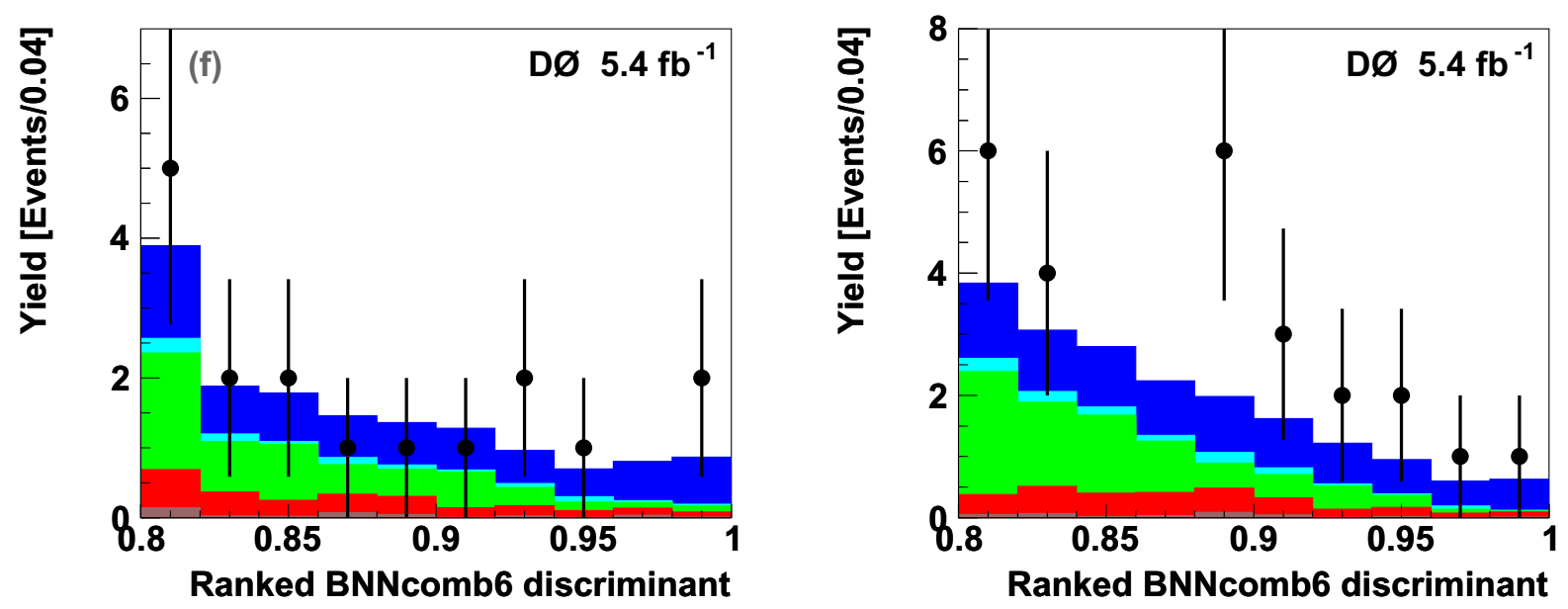

Figure 8.20: The BNNcombTSortZoom plots in the electron+muon channel for positive (left) and negative (right) events. 

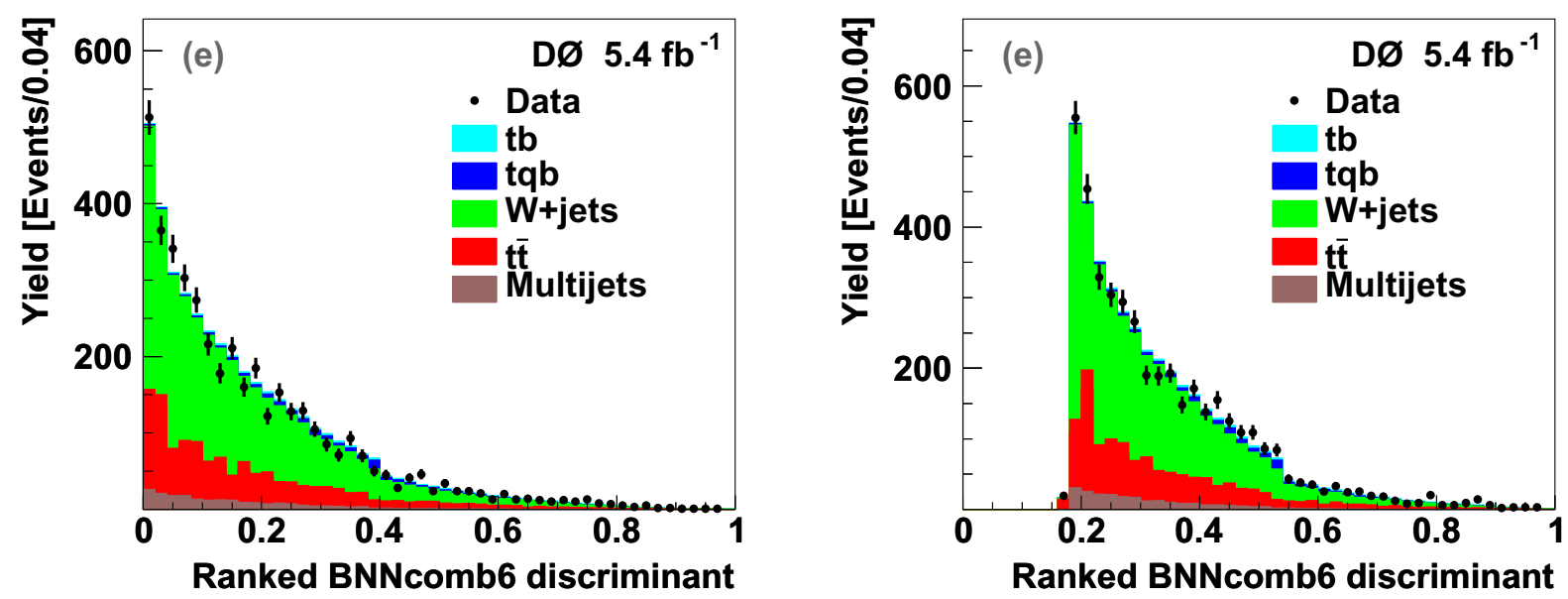

Figure 8.21: The $s$ discriminant plots in the electron+muon channel for positive (left) and negative (right) events.
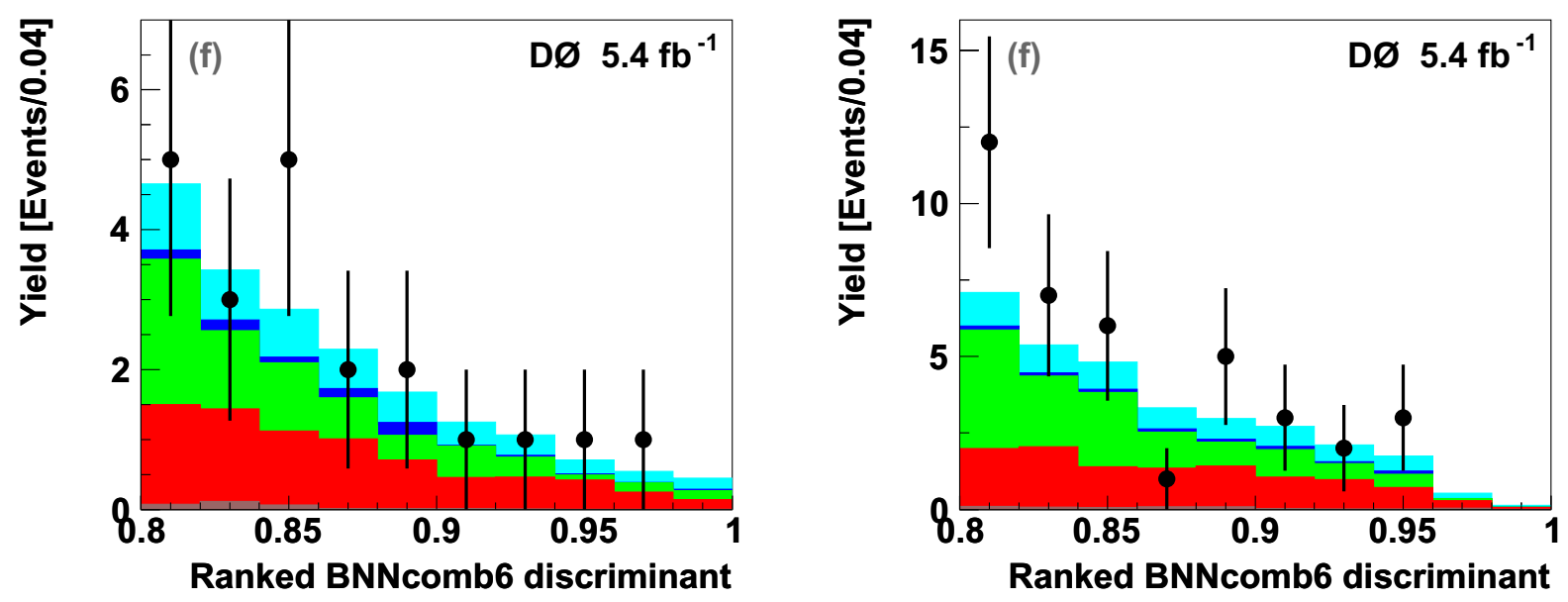

Figure 8.22: The BNNcombSSortZoom plots in the electron+muon channel for positive (left) and negative (right) events. 


\section{Chapter 9}

\section{Statistical Analysis}

In order to obtain a measurement of the $\mathrm{CP}$ asymmetry, the top and antitop cross sections need to be measured simultaneously. The cross sections are determined using the signal and background binned distributions of the combination BNN outputs. The cross section calculations are performed employing Bayesian statistics with the top_statistics [63] package.

\subsection{Poisson distribution likelihood}

The basic assumption of particle physics is that event counts follow a Poisson distribution, that is, if the expected number of events is $d$, the probability to observe a 
count of $D$, is given by:

$$
L(D \mid d)=\frac{e^{-d_{d} D}}{D !},
$$

The expected number of events $d$ come from signal and background sources:

$$
d=\alpha \mathcal{L} \sigma+\sum_{i=1}^{N} b_{i} \equiv a \sigma+\sum_{i=1}^{N} b_{i},
$$

where $\alpha$ is the signal acceptance, $\mathcal{L}$ is the integrated luminosity, $\sigma$ is the signal cross section (the quantity to be measured), $b_{i}$ the expected event count for background source $\mathrm{i}$, and $a \equiv \alpha \mathcal{L}$ is defined as the effective luminosity for the signal. $N$ is the total number of background sources.

We measure the likelihood for each individual histogram bin and regard them as independent measurement channels. The total likelihood for each analysis channel is thus a product of the likelihood of each histogram bin:

$$
L(\mathbf{D} \mid \mathbf{d}) \equiv L(\mathbf{D} \mid \sigma, \mathbf{a}, \mathbf{b})=\prod_{i=1}^{M} L\left(D_{i} \mid d_{i}\right),
$$

where $\mathbf{D}$ and $\mathbf{d}$ represent vectors of observed data counts and expected signal plus background event counts, and $\mathbf{a}$ and $\mathbf{b}$ are vectors of effective luminosity and backgrounds. $M$ is the total number of bins in the channel. 


\subsection{Bayesian analysis}

Bayes' theorem links the uncertainty of a probability model before and after observing the modelled system. From Bayes' theorem, we obtain:

$$
p(\sigma, \mathbf{a}, \mathbf{b} \mid \mathbf{D})=\frac{L(\mathbf{D} \mid \sigma, \mathbf{a}, \mathbf{b}) \pi(\sigma, \mathbf{a}, \mathbf{b})}{p(\mathbf{D})},
$$

We regard the probability to observe a given number of counts for data $p(\mathbf{D})$ is a constant. Further integrating over the parameters $\mathbf{a}$ and $\mathbf{b}$ gives the probability density for the signal cross section given observed event counts D:

$$
p(\sigma \mid \mathbf{D})=\frac{1}{\mathcal{N}} \iint L(\mathbf{D} \mid \sigma, \mathbf{a}, \mathbf{b}) \pi(\sigma, \mathbf{a}, \mathbf{b}) d \mathbf{a}, d \mathbf{b}
$$

where $\mathcal{N}$ is an overall normalization factor from the requirement $\int_{0}^{\sigma} \max p(\sigma \mid \mathbf{D}) d \sigma=$ 1 , where the integral upper bound $\sigma_{\max }$ is chosen as when the posterior probability density $p(\sigma \mid \mathbf{D})$ is sufficiently close to zero. The function $p(\sigma \mid \mathbf{D})$ is called the posterior probability density, which is the probability density of observing a certain cross section assuming event counts $\mathbf{D}$ is observed. The function $\pi(\sigma, \mathbf{a}, \mathbf{b})$ is called the prior probability density. $L(\mathbf{D} \mid \sigma, \mathbf{a}, \mathbf{b})$ is the Poisson likelihood detailed in last section.

In general, our prior knowledge of $\mathbf{a}$ and $\mathbf{b}$ is independent of that of cross section 
$\sigma$. Thus,

$$
\pi(\sigma, \mathbf{a}, \mathbf{b})=\pi(\mathbf{a}, \mathbf{b} \mid \sigma) \pi(\sigma)=\pi(\mathbf{a}, \mathbf{b}) \pi(\sigma)
$$

We assume that the prior probability density of signal cross section is evenly distributed, which is equivalent to a flat prior for the signal cross section: $\pi(\sigma)=$ $1 / \sigma_{\max }$, as shown in the Figure 9.1 below.

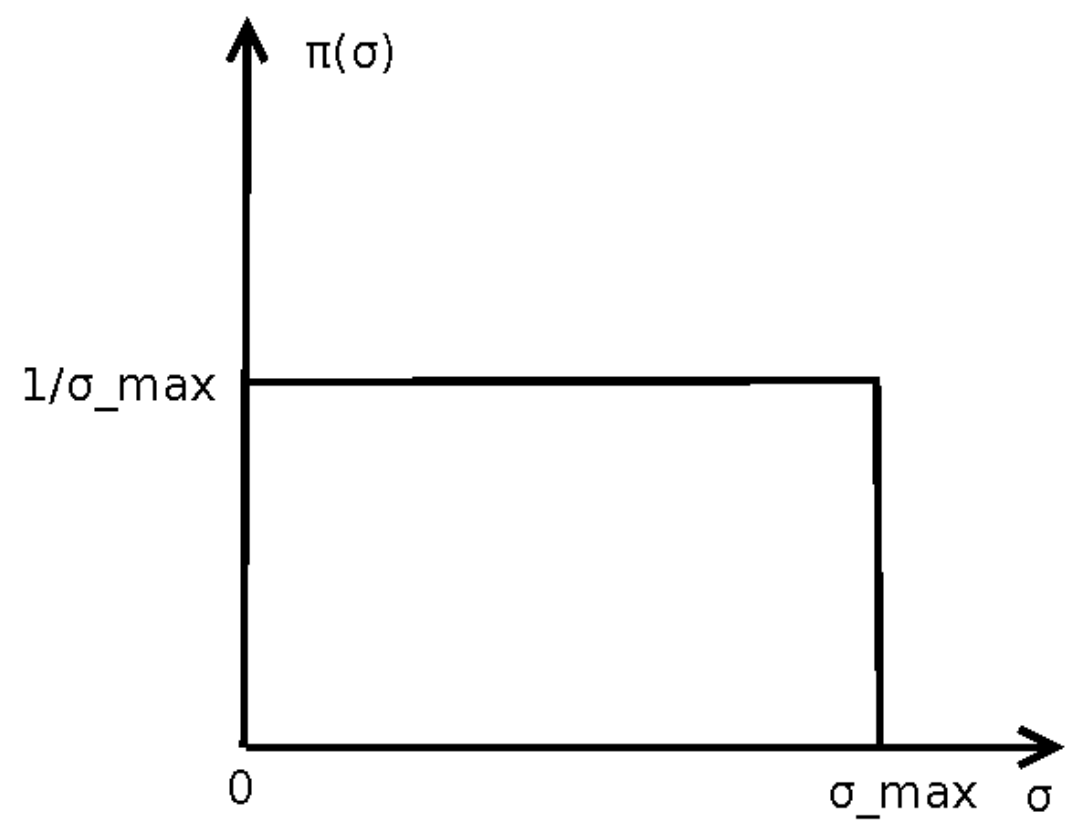

Figure 9.1: An illustrative plot of a flat prior probability density for the signal cross section.

Thus, the posterior probability density for the signal cross section Eq. 9.5 can be written as:

$$
p(\sigma \mid \mathbf{D})=\frac{1}{\mathcal{N} \sigma_{\max }} \iint L(\mathbf{D} \mid \sigma, \mathbf{a}, \mathbf{b}) \pi(\mathbf{a}, \mathbf{b}) d \mathbf{a}, d \mathbf{b}
$$


We choose the peak of the posterior density distribution as the measured cross seciton, and the $68 \%$ confidence interval about the peak as an estimate of its uncertainty, as shown in Figure 9.2.

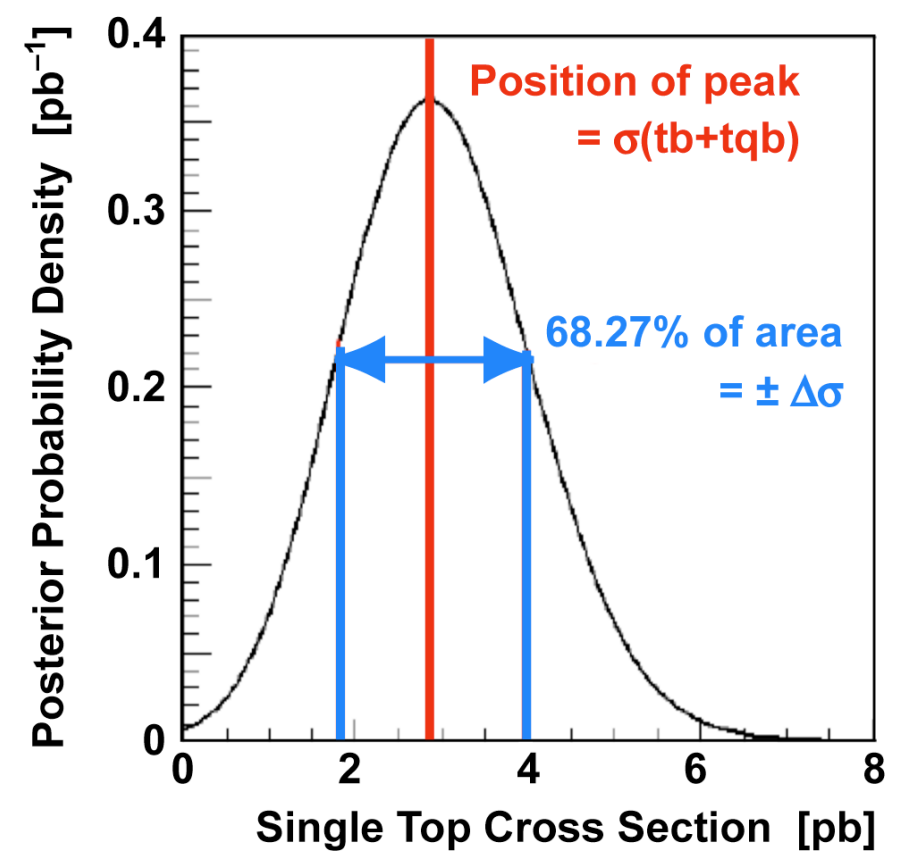

Figure 9.2: An illustrative plot demonstrating cross section measurement from a posterior probability density.

To measure top and antitop quark cross sections, we generalize the method above to measure simultaneously the cross sections of two signals, $\sigma_{+}$and $\sigma_{-}$, by building a two-dimensional posterior probability density. The expected event count in this case will be:

$$
d=a_{+} \sigma_{+}+a_{-} \sigma_{-}+\sum_{i=1}^{N} b_{i}
$$


where $a_{+}$and $a_{-}$are the effective luminosities of the two signals respectively.

As before, we assume a flat prior for each of the two signals:

$$
\pi\left(\sigma_{+}\right)=1 / \sigma_{1, \max }
$$

$$
\pi\left(\sigma_{-}\right)=1 / \sigma_{2, \max }
$$

where $\sigma_{1, \max }$ and $\sigma_{2, \max }$ is a sufficiently high upper bound for the first and second signal cross section to be close to zero.

Finally we get a two dimensional posterior probability density for different values of $\sigma_{+}$and $\sigma_{-}$:

$$
p\left(\sigma_{+}, \sigma_{-} \mid \mathbf{D}\right)=\frac{1}{\mathcal{N} \sigma_{1, \max } \sigma_{2, \max }} \iint L\left(\mathbf{D} \mid \sigma_{+}, \sigma_{-}, \mathbf{a}, \mathbf{b}\right) \pi(\mathbf{a}, \mathbf{b}) d \mathbf{a}, d \mathbf{b} .
$$

Replacing the likelihood with the Poisson distribution, we obtained

$$
L\left(\mathbf{D} \mid \sigma_{+}, \sigma_{-}, \mathbf{a}, \mathbf{b}\right)=\prod_{p=1} \frac{e^{-d_{p}} d_{p}^{D_{p}}}{\Gamma\left(D_{p}+1\right)} \prod_{n=1} \frac{e^{-d_{n}} d_{n}^{D_{n}}}{\Gamma\left(D_{n}+1\right)}
$$

where the $d_{p}$ and $d_{n}$ is the expected number of events with positive and negative 
leptons respectively in each bin:

$$
\begin{aligned}
& d_{p}=\sigma_{+} a_{p}+b_{p} \\
& d_{n}=\sigma_{-} a_{n}+b_{n}
\end{aligned}
$$

Here $a_{p}$ and $a_{n}$ represent the effective luminosities for single top and antitop, and $b_{p}$ and $b_{n}$ are the background yields for events with leptons with positive and negative charges, respectively.

$\pi(\mathbf{a}, \mathbf{b})$ encodes our knowledge of the effective signal luminosities and background yields. The posterior density distribution is obtained by creating a large number of samples of systematic shifts in $\mathbf{a}$ and $\mathbf{b}$. A separate likelihood distribution is obtained for each sample, and the final posterior is the sum over all of the individual likelihoods.

From this likelihood the $2 \mathrm{~d}$ posterior probability density Eq. 9.11 is rewritten as:

$\left.p\left(\sigma_{+}, \sigma_{-}\right)=\frac{1}{\mathcal{N} \sigma_{+, \max } \sigma_{-, \max }} \iint \prod_{p=1} \frac{e^{-d_{p} d_{p}^{D_{p}}}}{\Gamma\left(D_{p}+1\right)} \prod_{n=1} \frac{e^{-d_{n}} d_{n}^{D_{n}}}{\Gamma\left(D_{n}+1\right)} \pi(\mathbf{a}, \mathbf{b}) d \mathbf{a} d \mathbf{b} 9.14\right)$

We can then define a contour plot of equal probability that encloses a volume CL around the peak of the posterior density, as illustrated in Figure 9.3.

The one dimensional posterior probability density for each positive and negative signal can be derived by integrating the two-dimensional posterior density Eq. 9.11 


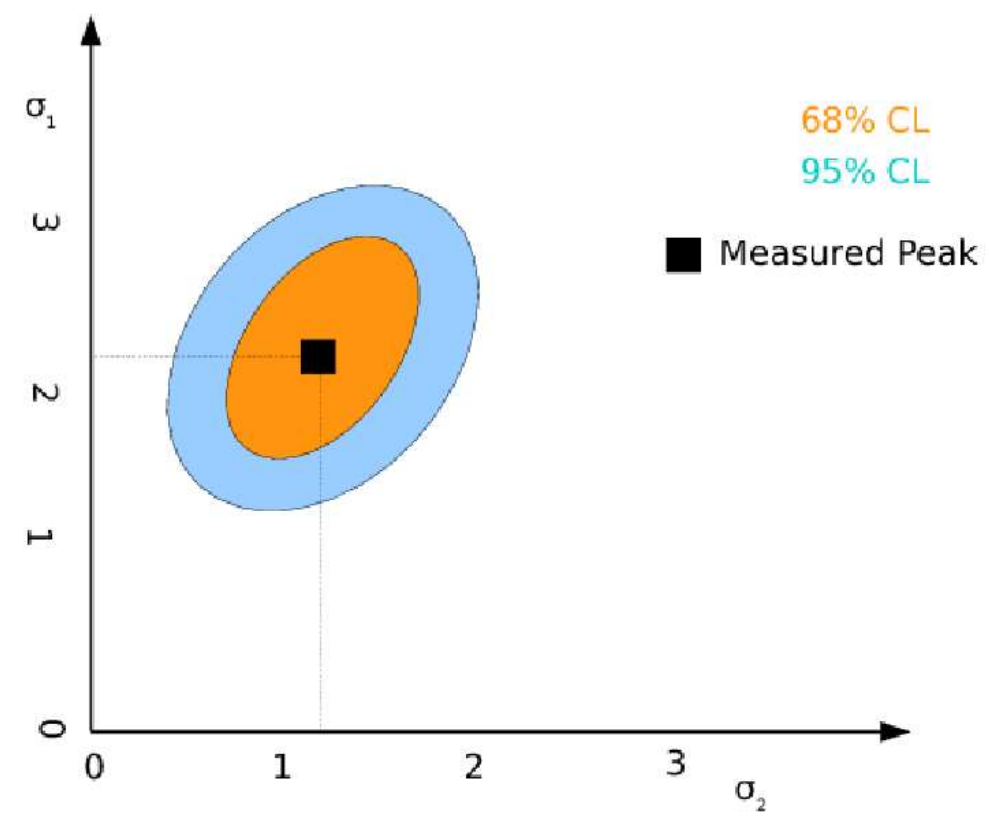

Figure 9.3: An illustrative plot demonstrating a contour plot derived from a two dimensional posterior probability density. 
over the other signal:

$$
p\left(\sigma_{+} \mid \mathbf{D}\right)=\int_{0}^{\sigma_{-}, \max } p\left(\sigma_{+}, \sigma_{-} \mid \mathbf{D}\right) d \sigma_{-},
$$

and

$$
p\left(\sigma_{-} \mid \mathbf{D}\right)=\int_{0}^{\sigma_{+}, \max } p\left(\sigma_{+}, \sigma_{-} \mid \mathbf{D}\right) d \sigma_{+} .
$$

Then we can determine the cross section measurements for positive and negative signals each in the same way as in the one dimensional case, as shown in Figure 9.2.

\subsection{Posterior probability density for the CP asym-}

\section{metry}

To obtain the posterior probability density for the asymmetry, we utilize the relationships (Eq. 2.2):

$$
\mathcal{A}=\frac{\sigma_{+}-\sigma_{-}}{\sigma_{+}+\sigma_{-}} \quad ; \quad \sigma=\sigma_{+}+\sigma_{-}
$$

where $\mathcal{A}$ is the definition of the top production asymmetry, and $\sigma$ the total production cross section. 
Combining with Eq. 9.14, we obtain

$$
p(\sigma, \mathcal{A})=\frac{\sigma}{2 \mathcal{N} \sigma_{\max }} \int L(\mathbf{D} \mid \sigma(1+\mathcal{A}) / 2, \sigma(1-\mathcal{A}) / 2, \mathbf{a}, \mathbf{b}) \pi(\mathbf{a}, \mathbf{b}) d \mathbf{a} d \mathbf{b}
$$

The posterior probability density for the asymmetry $p(\mathcal{A})$ thus can be computed by integrating over the total cross section $\sigma$ :

$$
p(\mathcal{A})=\int p(\sigma, \mathcal{A}) d \sigma
$$




\section{Chapter 10}

\section{Systematic Uncertainties}

Systematic uncertainties expresses our inability to model the detector accurately. Systematic errors are often difficult to assess, because they do not show up as fluctuations in the results of repeated measurements, as statistical uncertainties do. It is important to think about possible sources of systematic errors and to try to correct them or rule them out.

Systematic uncertainties enter the single top measurements in two ways: as uncertainty on the normalization of the signal and background samples, and as effects that change the shapes of the distributions of those samples and the shapes of the expected signal distributions, i.e. the combination BNN outputs. 


\subsection{Uncertainties affecting normalization only}

These systematic uncertainties affects only the normalization of the signal and background samples.

\section{- Integrated luminosity}

The $6.1 \%$ uncertainty on the luminosity estimate affects the signal, $t \bar{t}, Z+$ jets, and diboson yields.

\section{- Theory cross sections}

The uncertainties on the single top and $t \bar{t}$ cross sections come from Refs. [1] and [64], and are calculated for 3 top quark mass points: $170 \mathrm{GeV}, 172.5 \mathrm{GeV}$, and $175 \mathrm{GeV}$. The resulting values for the uncertainties for $172.5 \mathrm{GeV}$ are $\pm 3.8 \%$ for s-channel $t b, \pm 5.3 \%$ for t-channel $t q b$, and $+6.4 \%,-9.0 \%$ for $t \bar{t}$. For $t b+t q b$ combined, the uncertainty is $\pm 4.8 \%$. (NB, the uncertainty on the signal cross section does not of course enter the experimental measurement of it.) The uncertainties on the $Z$ +jets cross section is set to $3.3 \%$ as per reference [65]. The uncertainties on the diboson cross sections is set to $7 \%$ in accordance with the Higgs group current practice [66].

\section{- Branching fractions}

From the Particle Data Book, the branching fractions for a $W$ boson to decay to an electron, muon, or tau lepton, have an average uncertainty of $1.5 \%$ and 
we include this in the MC normalization uncertainties.

\section{- Parton distribution functions}

We have estimated the effect of changing the parton distribution functions on the signals and assign a systematic uncertainty of $2 \%$ to the signal acceptances from this source. PDF uncertainties on the acceptance can be calculated by reweighting the $\mathrm{MC}$ events before selection using 40 CTEQ PDF sets and then calculating the acceptance for each of the eigen sets [50]. The square root of the sum of the squares of the difference between the PDF eigen sets with the nominal PDF set is quoted as uncertainty.

\section{- Trigger efficiency}

We use an OR of many trigger conditions which give us a trigger efficiency of close to $100 \%$. We assign an uncertainty of $5 \%$ to the trigger efficiency in all channels and treat it as uncorrelated between p17 and p20.

\section{- Instantaneous luminosity reweighting}

We reweight the instantaneous luminosity distributions of all MC samples to make them match Run IIa or Run IIb data distributions as appropriate. The uncertainty on this reweighting is $1.0 \%$.

\section{- Primary vertex modeling and selection}

We reweight the distribution of the primary vertices along the beamline in $\mathrm{MC}$ 
to match that in data [67], [68]. The uncertainty on this reweighting is $0.05 \%$ (negligible). The uncertainty on the difference in primary vertex selection efficiency between data and MC is $1.4 \%$.

\section{- Color Reconnection}

We estimate the systematics due to color reconnections by taking the difference in signal efficiency between a sample generated with PYTHIA TuneAPro and PYTHIA TuneACPro [69]. The relative difference on preselection and btagging efficiency between both simulations is found to be $1.0 \%$ and is assigned as an additional uncertainty to the central efficiencies for $t \bar{t}$ background and signal MC.

\section{- Relative b/light jet response}

This uncertainty takes into account the difference between the nominal inclusive jet response and the response for B- hadrons. To estimate this difference, particle jets in a $t \bar{t}$ lepton+jets sample were constructed and classified as b- or light jets. Single particle response curves for both data and MC were then applied to the particle jets to predict the energy of a reconstructed jet in the calorimeter. From these reconstructed energies, the ratio $p_{T}^{d a t a} / p_{T}^{M C}$ was then calculated separately for both b-jets and light jets and the double ratio 
evaluated:

$$
\frac{\left(p_{T}^{\text {data }} / p_{T}^{\mathrm{MC}}\right)_{\mathrm{b}-\text { jet }}}{\left(p_{T}^{\text {data }} / p_{T}^{\mathrm{MC}}\right)_{\text {light jet }}}
$$

resulting in a differnce of $1.8 \%$ [70]. The systematics uncertainty is then obtained by shifting down by $1.8 \%$ the nominal response of b-jets (identified based on the MC truth information).

\section{- Electron reconstruction and identification efficiency}

The electron scale factor uncertainty is taken from Ref. [55], but adapted to the electron acceptance cuts used in this analysis in accordance with [71]. The uncertainty includes contributions from the fit used to evaluate the background in the $Z$ boson sample, as well as dependencies on the luminosity, distance to closest jet, jet multiplicity, and electron $p_{T}$. The resulting total uncertainty is $2.8 \%$ for $\mathrm{p} 17$, and $3.8 \%$ for $\mathrm{p} 20$ data.

\section{- Muon reconstruction and identification efficiency}

The muon scale factor uncertainties for muon reconstruction (1.4\%), muon identification (1.2\%) and muon isolation (0.4\%) are taken from Table 6, 4 and 8 of Ref. [72]. They were estimated by the muon ID group, for the tag/probe method, background subtraction, and limited statistics in the parameterization. An additional uncertainty of $1 \%$ is added in quadrature to take into account the latest muon ID as explained in Appendix 5.1. The assigned total uncertainty 
is $2.1 \%$.

\section{- Jet Fragmentation and higher-order effects}

We have measured the systematic uncertainty due to jet fragmentation by comparing the acceptance of the nominal $t \bar{t}$ events generated with ALPGEN+PYTHIA (as used in the analysis) to ones generated with ALPGEN+HERWIG. The resulting uncertainty of $0.7 \%, 3.7 \%$ and $4.7 \%$ for events with 4,5 and 6 jets, is applied to the signal samples in the 2,3 and 4 jet bin, respectively.

We have also measured the systematic uncertainty due to a combination of jet fragmentation and higher order effects (We take the variations observed in the top pair cross section analysis described in $D \varnothing$ Note 6025 , averaged over run

period and lepton type.) comparing the acceptance of the nominal $t \bar{t}$ sample to ones generated with MC@NLO+HERWIG. The resulting uncertainty is $1.6 \%$ for events with 2 jets, $1.7 \%$ for events with 3 jets, and $7.0 \%$ for events with 4 jets. The variation is anti-correlated between events with 2 or 3 jets and events with 4 jets. This uncertainty is applied exclusively to the $t \bar{t}$ sample and accounts for both jet fragmentation and higher order effects.

\section{- Initial-state and final-state radiation}

We use $t \bar{t}$ samples that were generated varying the ISR/FSR settings and we have evaluated this uncertainty in those samples as follows: for signal MC, which have 2,3 , or 4 jets, we take the uncertainties from the $t \bar{t}$ events with 4 , 
5, 6 jets. For the $t \bar{t}$ background, we take numbers from the $t \bar{t}$ samples with 2, 3, and 4 jets directly. All uncertainties are averaged over the electron and muon channels, and are half the difference between the ISR and FSR samples. We find an uncertainty ranging from $0.8 \%$ to $10.9 \%$ and apply it to signal and $t \bar{t}$ samples.

\section{- b-jet fragmentation}

The size of the uncertainty from the $b$-jet modeling was evaluated in the $t \bar{t}$ pairs cross section analysis following the method described in Ref. [70]. The uncertainty arises from the difference between the fragmentation parameterizations preferred by SLD vs. LEP data. A $2.0 \%$ value is measured and applied to signal, $t \bar{t}$ and $Z$ bb samples.

\section{- Taggability}

The uncertainty associated with $b$-tagging in $\mathrm{MC}$ events includes components for the taggability and the tagging efficiency. The taggability uncertainties are evaluated by raising and lowering the taggability scale factor values by one standard deviation on each MC sample and repeating the entire analysis. The taggability uncertainties affect the normalization of the MC samples only and the values are between $3.1 \%$ and $21.5 \%$.

\section{- $\mathrm{W}+$ jets heavy-flavor scale factor correction}


The heavy-flavor scale factor correction, $\lambda_{H F}$, for $W b \bar{b}$ and $W c \bar{c}$ is measured in a sample that is orthogonal to the one in the analysis and found to be consistent with 1 , with a $12 \%$ uncertainty. Two other methods based on the candidate sample also confirm that $\lambda_{H F}$ is consistent with one.

\section{- $\mathrm{Z}+$ jets heavy-flavor scale factor correction}

The heavy-flavor scale factor $K_{H F}^{\prime}$ for $Z b \bar{b}$ and $Z c \bar{c}$ is determined from NLO calculations, and we set the correction to it, $\lambda_{H F}$ to be 1.0 (since we cannot normalize to data to check it) with an uncertainty of $12 \%$, taken from the $\lambda_{H F}$ for $W+$ jets events.

\section{- $\mathrm{W}+$ jets and multijets normalization}

We use a fit to the pretagged data to determine the $W+$ jets and multijets background normalizations. The uncertainties are estimated to be $30 \%$ for the multijets backgrounds (40\% for the Run IIb MU channel), and $1.8 \%$ for the $W+$ jets backgrounds.

\section{- Lepton Charge Mis-Identification}

The CP violation measurement needs to separate the samples into positive and negative subsets, according to the lepton charge in the final state. We select the $Z \rightarrow e e$ or $Z \rightarrow \mu \mu$ events to measure the charge mis-ID rate. We require each event to have exactly two electrons or two muons, and the invariant mass 
of the leptons between 80 and $100 \mathrm{GeV}$ ( $Z$ peak). We measured the fake rate below $1 \%$. And since we are not sensitive to effects of the order of $1 \%$ compared to the existing much larger systematic uncertainties, we decide to simply add a systematic uncertainty of $1 \%$ anti-correlated between the positive and negative charge samples. Details see Appendix D.

\section{- Sample statistics}

The MC and data samples we use to estimate the signal and background shapes are limited in size. As In particular the multijets background samples have low statistics after $b$ tagging. We take the background sample statistics into account for each sample in each bin of the final discriminant distribution.

\subsection{Uncertainties affecting normalization and shape}

The following systematics affects the shapes of the distributions of the signal and backgrounds, and the shapes of the combination BNN outputs.

\section{- Jet reconstruction and identification}

The efficiency to reconstruct and identify jets has an uncertainty varying from $0.04 \%$ to $3.7 \%$ for all MC samples. 


\section{- Jet energy resolution}

We assign a normalization and shape-changing uncertainty ranging from $0.2 \%$ to $11.6 \%$ as the jet energy resolution uncertainty for all signals and backgrounds.

\section{- Jet energy scale}

The JES correction is raised and lowered by one standard deviation on each MC sample and the whole analysis repeated, which produces a shape-changing uncertainty, and an overall normalization uncertainty. The normalization part ranges from $0.3 \%$ to $14.6 \%$ for all MC samples.

\section{- Vertex confirmation}

The efficiency to identify vertex-confirmed jets has an uncertainty varying from $0.1 \%$ to $9.6 \%$ for all MC samples.

\section{- $b$-tagging}

The uncertainty associated with $b$-tagging in MC events includes scale factor uncertainties $(\mathrm{SF})$ in the case of applying direct tagging and tag rate (TRF) uncertainties in the case of using random tagging. This is done simultaneously for heavy flavor and light jets, as it was done in the single top observation analysis. The SF and TRF uncertainties originate from several sources: statistical errors of MC event sets; the assumed fraction of heavy flavor in the multijets 
$\mathrm{MC}$ events for the mistag rate determination; and the parameterizations. The SF and TRF uncertainties affect both shape and normalization of the MC samples. The values range from $4.3 \%(5.8 \%)$ to $14.0 \%(11.2 \%)$ for single-tagged (double-tagged) samples.

\section{- $V+$ jets angular corrections}

We reweight the ALPGEN $W$ and $Z+$ jets background based on several pretagged data distributions. The uncertainty from these reweightings affects the shapes of the $W$ and $Z+$ jets background components. The reweighting procedure does not change the normalization of the $W$ and $Z+$ jets samples before $b$-tagging. After $b$-tagging, small normalization variations $(\approx 0.3 \%)$ are introduced by taggability and $b$-tagging scale factors.

\section{- Response to $b$ and $\bar{b}$ quarks}

The measurement of the top-antitop cross section difference could be affected by a different response of the calorimeter to quark and antiquark jets. Such a bias could come from a different calorimeter response to the $b$ and the $\bar{b}$ decays, respectively. To estimate this uncertainty, we rescale the $\mathrm{pT}$ (the 4 -vectors accordingly) of the $b$ and $\bar{b}$ by a factor of 0.9971 and 1.0021 respectively [73],

depending on $b$ or $\bar{b}$, and then re-apply the MVA filters. The measured effect is small, and we add another $1 \%$ flat systematic uncertainty to account for it. See Appendix F for details. 


\section{Chapter 11}

\section{CP Violation Measurement}

This chapter discusses the final cross section measurements for both top and antitop quarks, and the expected and observed CP asymmetry measurement results for all single top production channels, i.e. $s$-channel, $t$-channel and $s+t$-channel.

\subsection{Cross section measurement}

As can be seen from Section 2.4, to measure the CP Violation in single top, we must measure the cross sections separately both for top and antitop quarks. The cross section is determined in a Bayesian approach using the top_statistics software package [63]. The inputs are the BNN combined output distributions shown in Section 8.7.

These split MVA outputs are further fed into the top_statistics package to 
obtain the desired posteriors.

The cross section is measured by forming a two-dimensional $(2 d)$ posterior as a function of both the positive and the negative top cross sections. In this approach, the $2 d$ posterior is constructed with no theoretical assumption on either the positive or negative cross sections. From this $2 \mathrm{~d}$ posterior, we extract the individual positive (negative) cross section by integrating over the negative (positive) axis. Figure 11.1 shows an example of a $2 \mathrm{~d}$ posterior as a function of both the positive and the negative top cross sections.

The procedure is done separately for $s+t$ (considering $s+t$ as signal and all other contributions as background), $s$ (considering s as signal and adding the $t$ contribution to the backgrounds), and for $t$ (considering t as signal and adding the $s$ contribution to the backgrounds), in order to obtain a posterior density curve as function of the cross section asymmetry (defined in Section 2.4) for each individual channel. The central value of the asymmetry is taken from the peak (mode) in the posterior density function, and the $68 \%$ interval about the peak gives the uncertainty. One can see an example of this measurement from the illustrative Figure 11.2.

The next two chapters show the expected and observed results of the CP asymmetry measurement. Appendix ?? gives more supplement plots and results. 


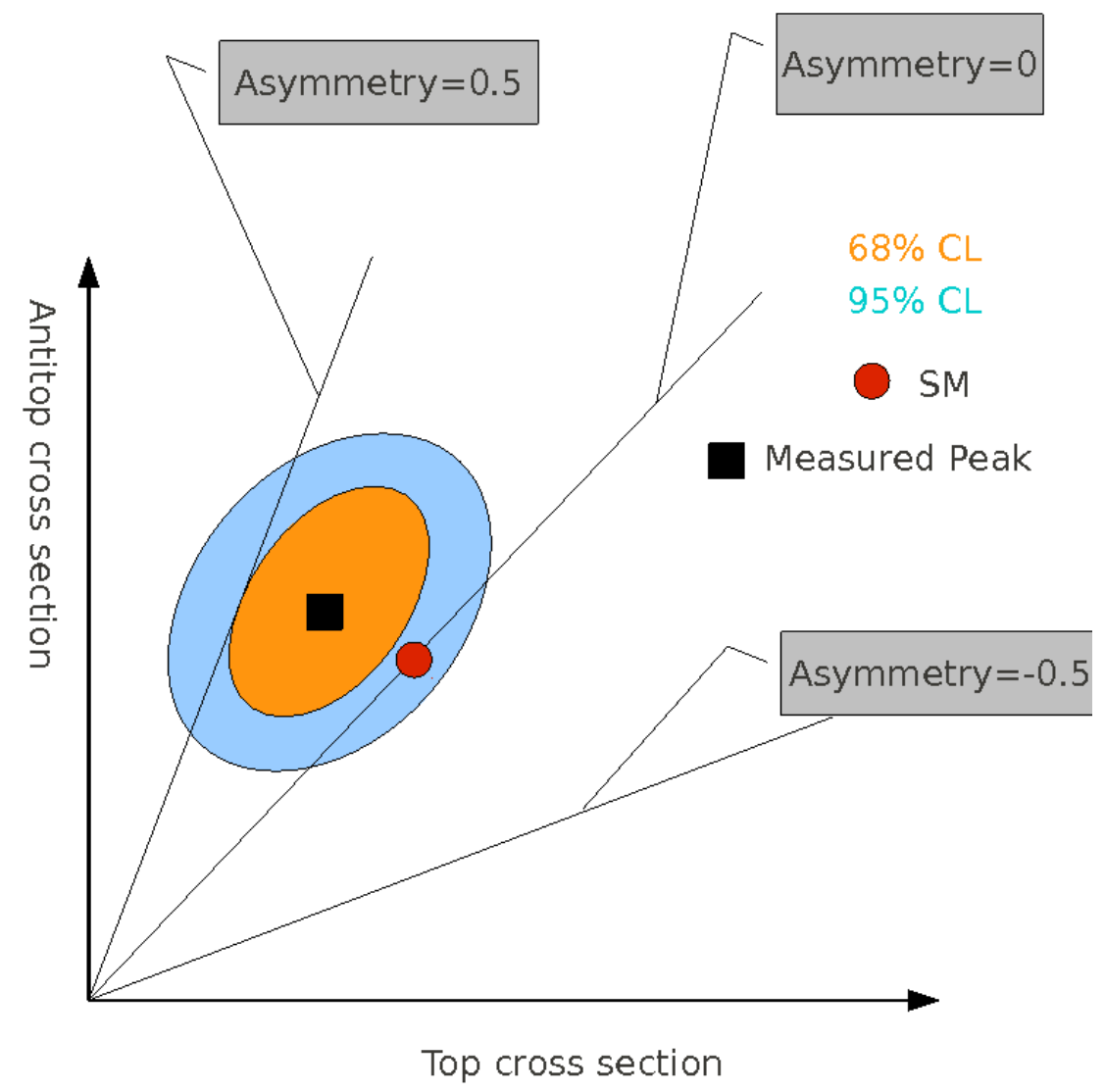

Figure 11.1: An illustrative example demonstrating a $2 d$ posterior as a function of both the positive and the negative top cross sections. This plot is obtained using the top_statistics package. The red small square denotes the Standard Model prediction (no CP violation). The black dot is the peak of the posterior which is close to our final results of top-antitop quark cross sections. The $68 \%, 90 \%$, and 95\% Confidence Limit (C.L.) are shown in contours of various colors. We also draw a diagonal line to visually show, how far away the measured peak is from CP symmetry, and whether it is shifted to the positive or negative side. 


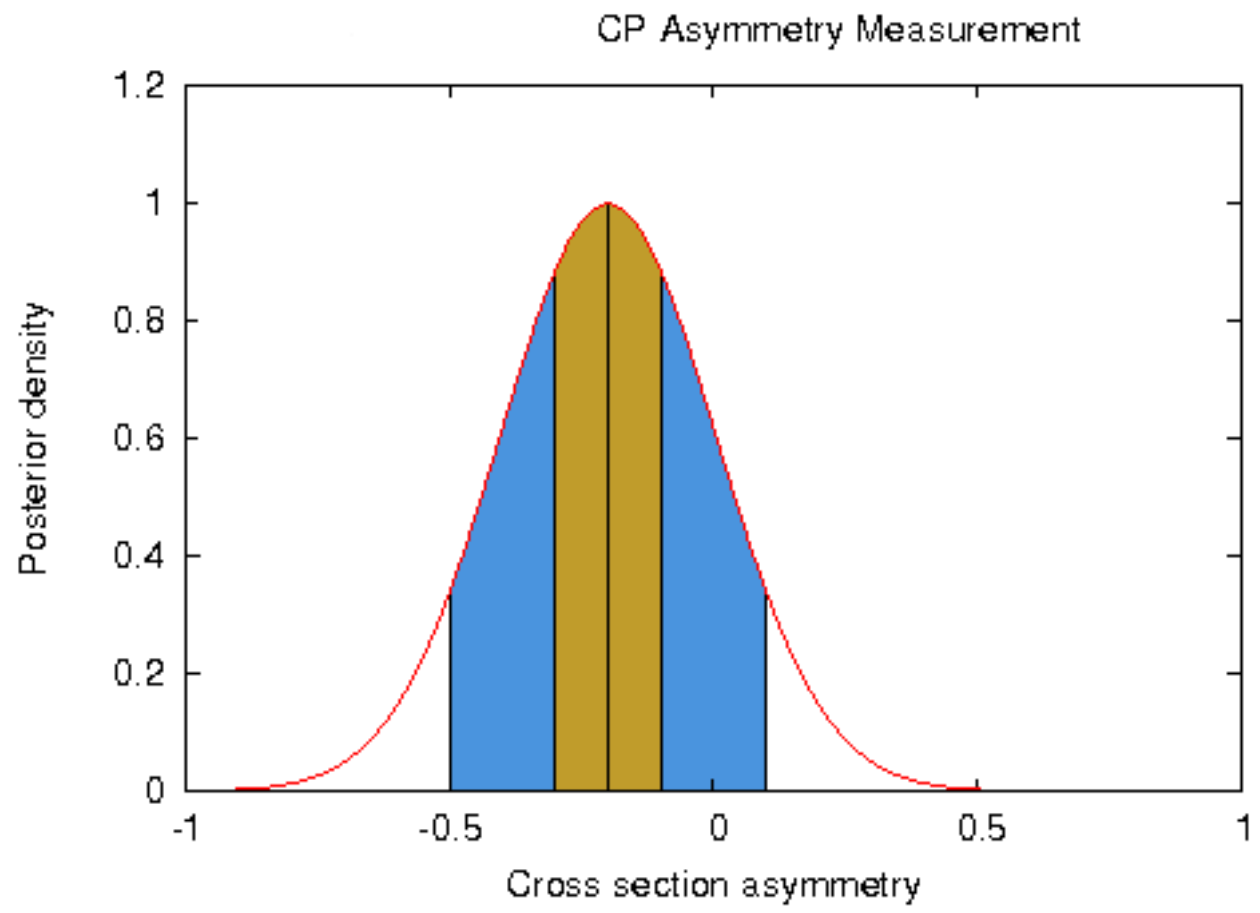

Figure 11.2: An illustrative example demonstrating how to measure the CP violation from an asymmetry posterior. This plot is obtained using the top_statistics package. We take the peak of the plot as the central value of the $\mathrm{CP}$ violation. The yellow area denotes the $68 \%$ CL interval, which is taken as the uncertainty of the CP violation measurement. We also draw a 95\% CL area with the light blue color. 


\subsection{Expected results}

The expected cross section is measured by setting the number of data events in each analysis channel to the expected number of background events plus the number of signal events, predicted when using the SM cross section value of $0.52 \mathrm{pb}$ for $s$-channel, $1.13 \mathrm{pb}$ for $t$-channel, and $1.65 \mathrm{pb}$ for $s+t$-channel (for a top mass of 172.5 GeV). The expected CP asymmetries are further calculated from the cross section measurements.

The posterior density functions for the $2 d$, single top production asymmetry, and cross sections, separately for top and antitop quarks, are shown in Figs. 11.3 to 11.5 taking into account all systematics.

From all the plots, we can see in each analysis channel, the expected cross section is very close to the standard model prediction, and thus the expected CP asymmetry is close to zero. This means the systematics do not bias our measurement.

Expected results for CP asymmetry in $s, t, s+t$ channels for different systematics are shown in Table 11.1.

The expected values are zero within large errors. The error is smallest in the $s+t$ channel, and largest in the $s$ channel. 
h2
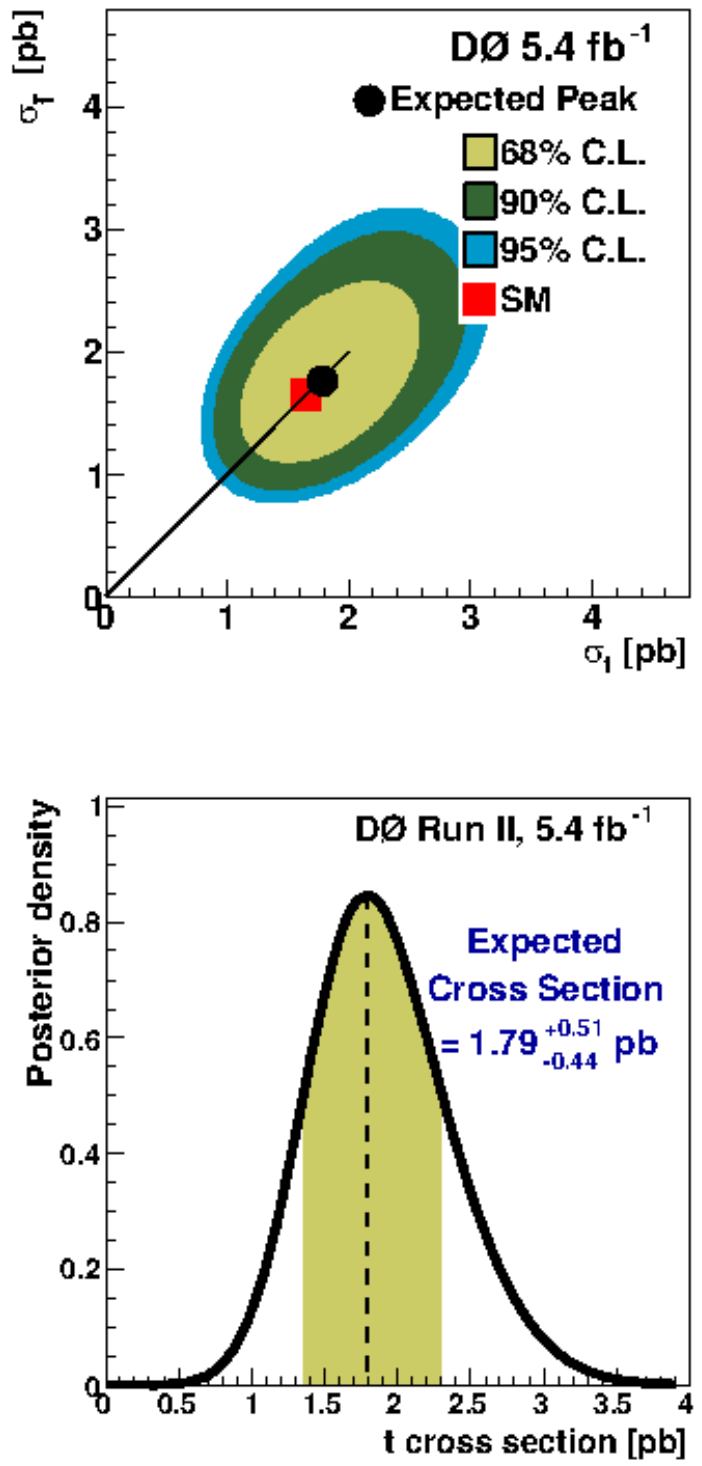
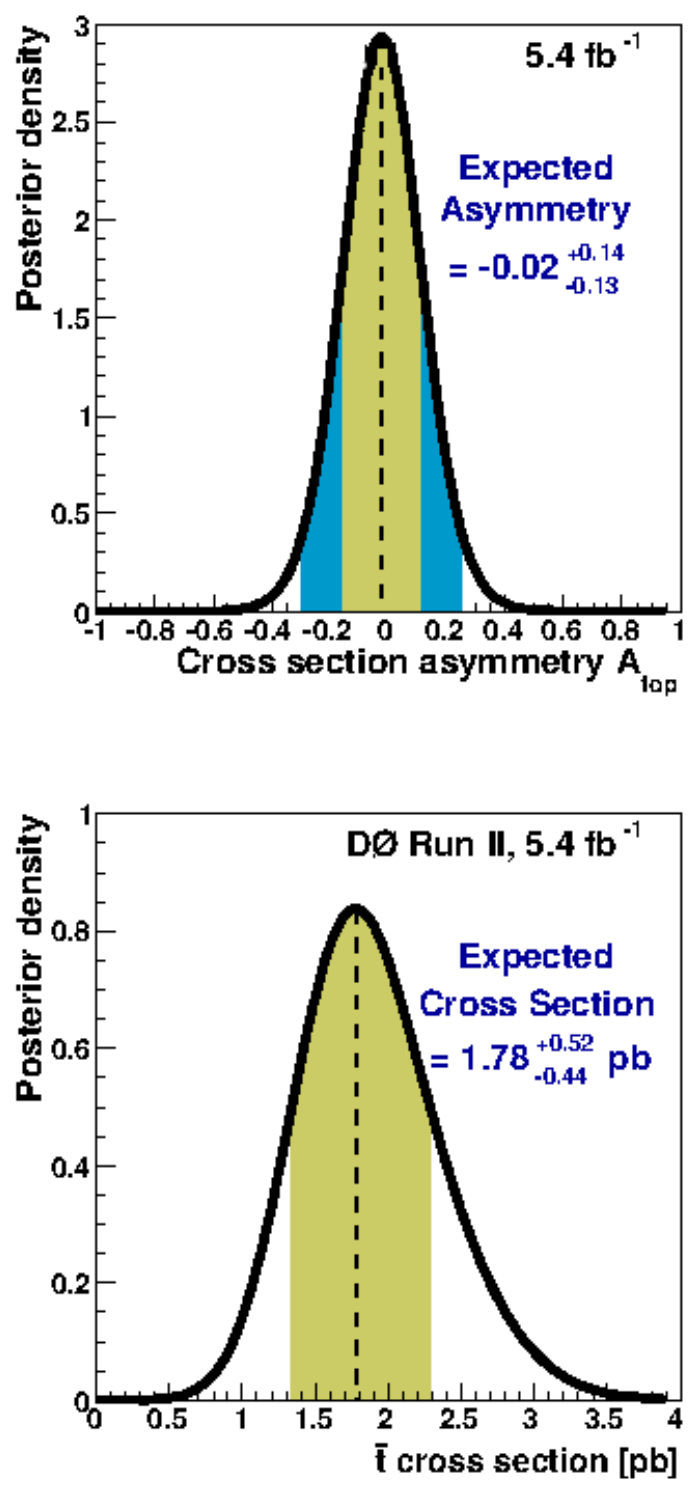

Figure 11.3: Expected posterior density distributions and measurements of top and anti-top cross sections in the $s+t$-channel. The top left plot shows the $2 d$ posterior of top and antitop production cross sections; top right plot shows the posterior of the top-antitop production cross section assymmetry $A_{t o p}$; bottom left plot shows the posterior of the top production cross section; bottom right plot shows the posterior of the antitop production cross section. The top (antitop) cross section are extracted by integrating over the antitop (top) axis respectively from the $2 \mathrm{~d}$ posterior. 
h2
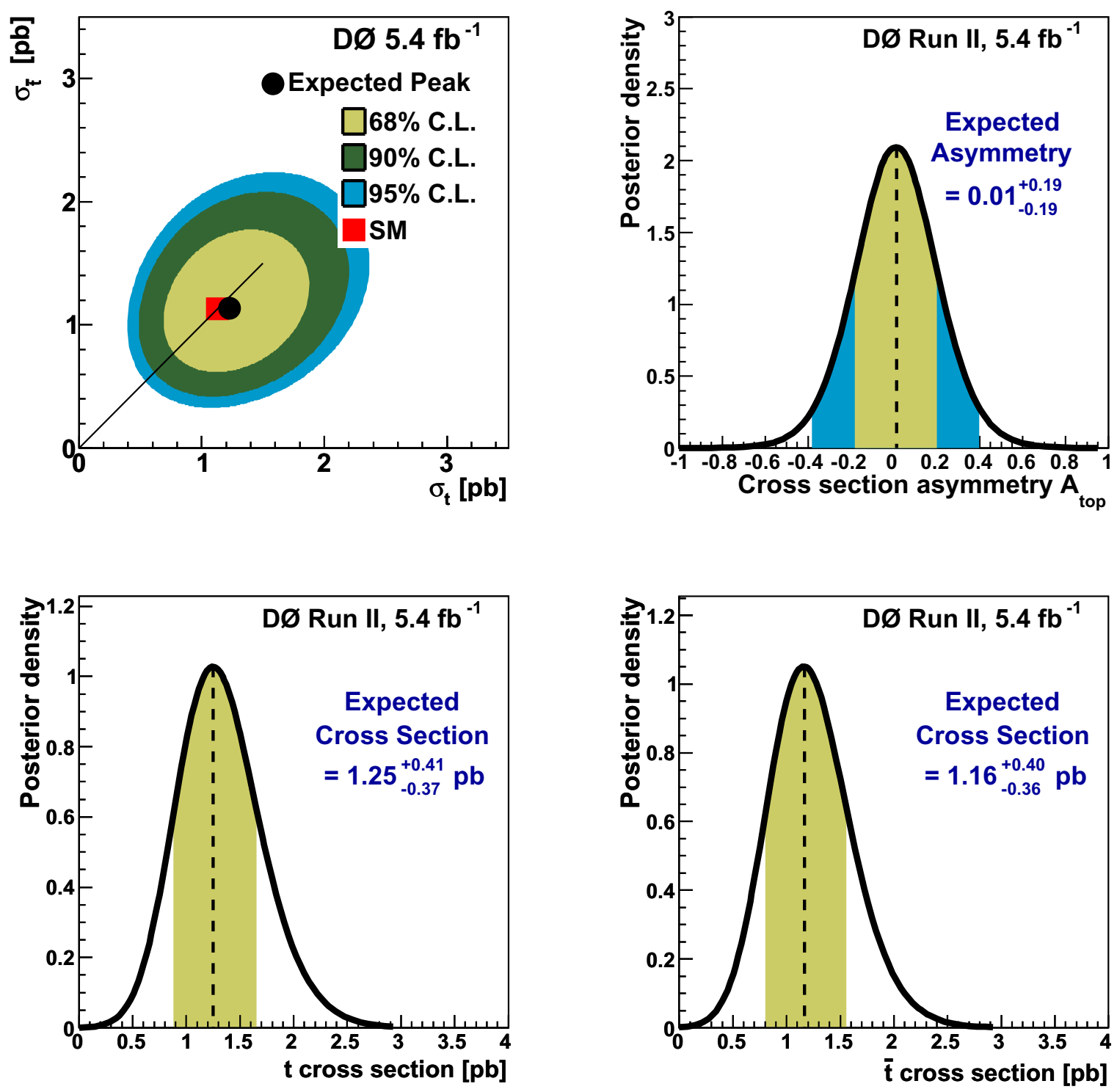

Figure 11.4: Expected posterior density distributions and measurements of top and anti-top cross sections in the $t$-channel. The top left plot shows the $2 d$ posterior of top and antitop production cross sections; top right plot shows the posterior of the top-antitop production cross section assymmetry $A_{t o p}$; bottom left plot shows the posterior of the top production cross section; bottom right plot shows the posterior of the antitop production cross section. The top (antitop) cross section are extracted by integrating over the antitop (top) axis respectively from the $2 \mathrm{~d}$ posterior. 
h2
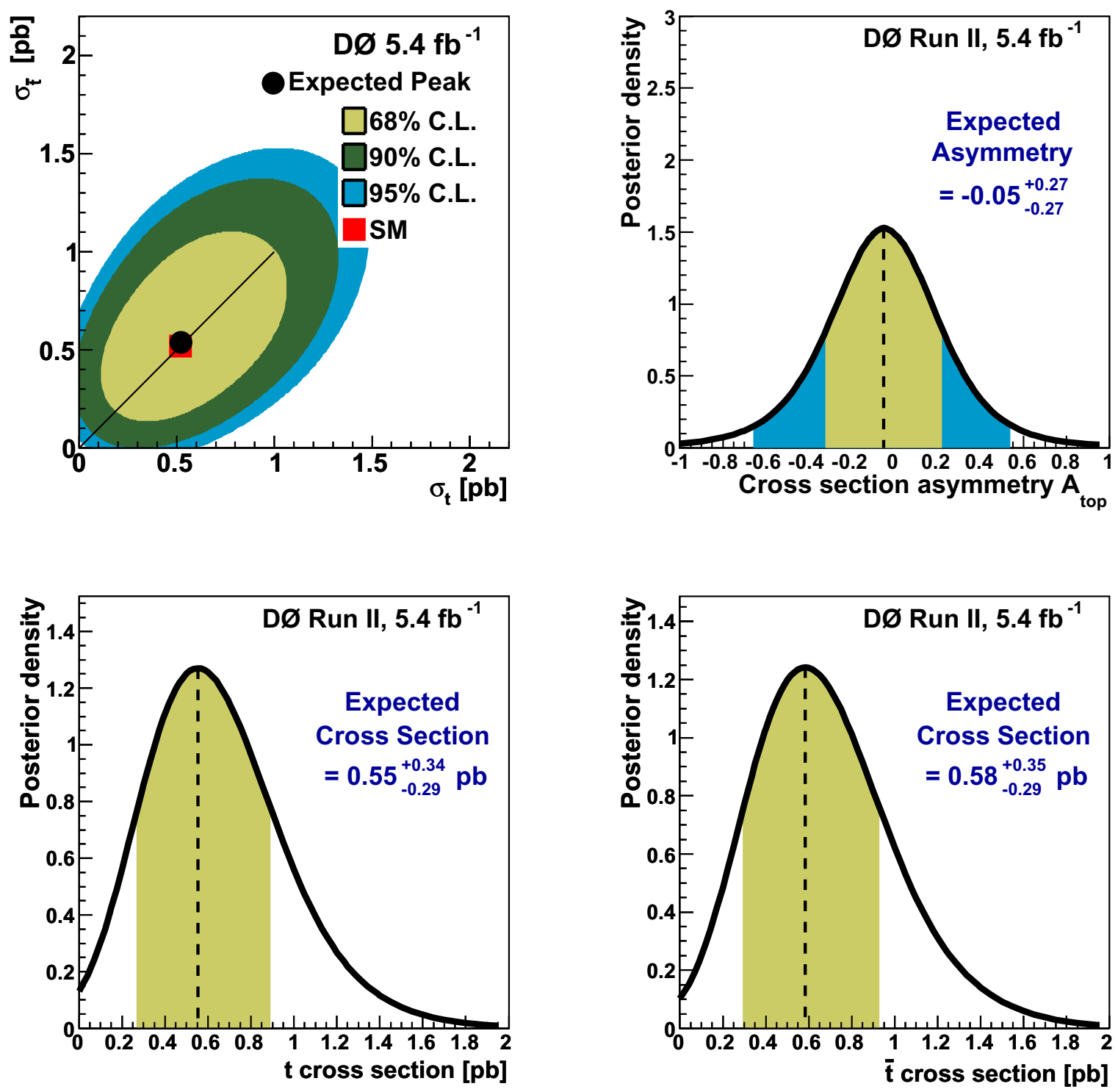

Figure 11.5: Expected posterior density distributions and measurements of top and anti-top cross sections in the $s$-channel. The top left plot shows the $2 d$ posterior of top and antitop production cross sections; top right plot shows the posterior of the top-antitop production cross section assymmetry $A_{t o p}$; bottom left plot shows the posterior of the top production cross section; bottom right plot shows the posterior of the antitop production cross section. The top (antitop) cross section are extracted by integrating over the antitop (top) axis respectively from the $2 \mathrm{~d}$ posterior. 
Table 11.1: Expected results for CP asymmetry in s, t, s+t channels.

\begin{tabular}{c|c} 
Discriminant & CP Asymmetry \\
\hline$t b$ & $-0.05_{-0.27}^{+0.28}$ \\
$t q b$ & $0.02_{-0.19}^{+0.19}$ \\
$t b+t q b$ & $-0.02_{-0.13}^{+0.14}$ \\
\hline
\end{tabular}




\subsection{Observed results}

The approach described in Sec. 11.2 is repeated using the data events to obtain the observed cross sections. The observed CP asymmetries are further calculated from the cross section measurements.

The posterior density functions for the $2 d$, single top production asymmetry, and cross sections separately for top and antitop quarks, are shown in Fig. 11.6 to Fig. 11.8 taking into account of all systematics. The colors show the $68 \%, 90 \%$, and 95\% CL regions.

Unlike the expected results, we can see the measured peak is shifted farther away from the Standard Model prediction, mostly around one standard deviation away. The $s$-channel deviates more than the $t$-channel, which is again larger than $s+t$ combined. The measured asymmetries all deviate in the same direction, i.e. to the negative part. This might be due to data fluctuation. We expect this bias will disappear as we have more statistics of events. Our analysis currently is still background dominated (S:B approximately 1:20). The $2 d$ plots show that the measured values are about one standard deviation away from the SM, while the asymmetry plot shows they are more than one standard deviation away. This is due to the integration in top_statistics, when converting the $2 d$ plot into $1 d$ asymmetry plot. We need at least three standard deviation to claim evidence, so we don't observe CP violation yet at this point. 
h2
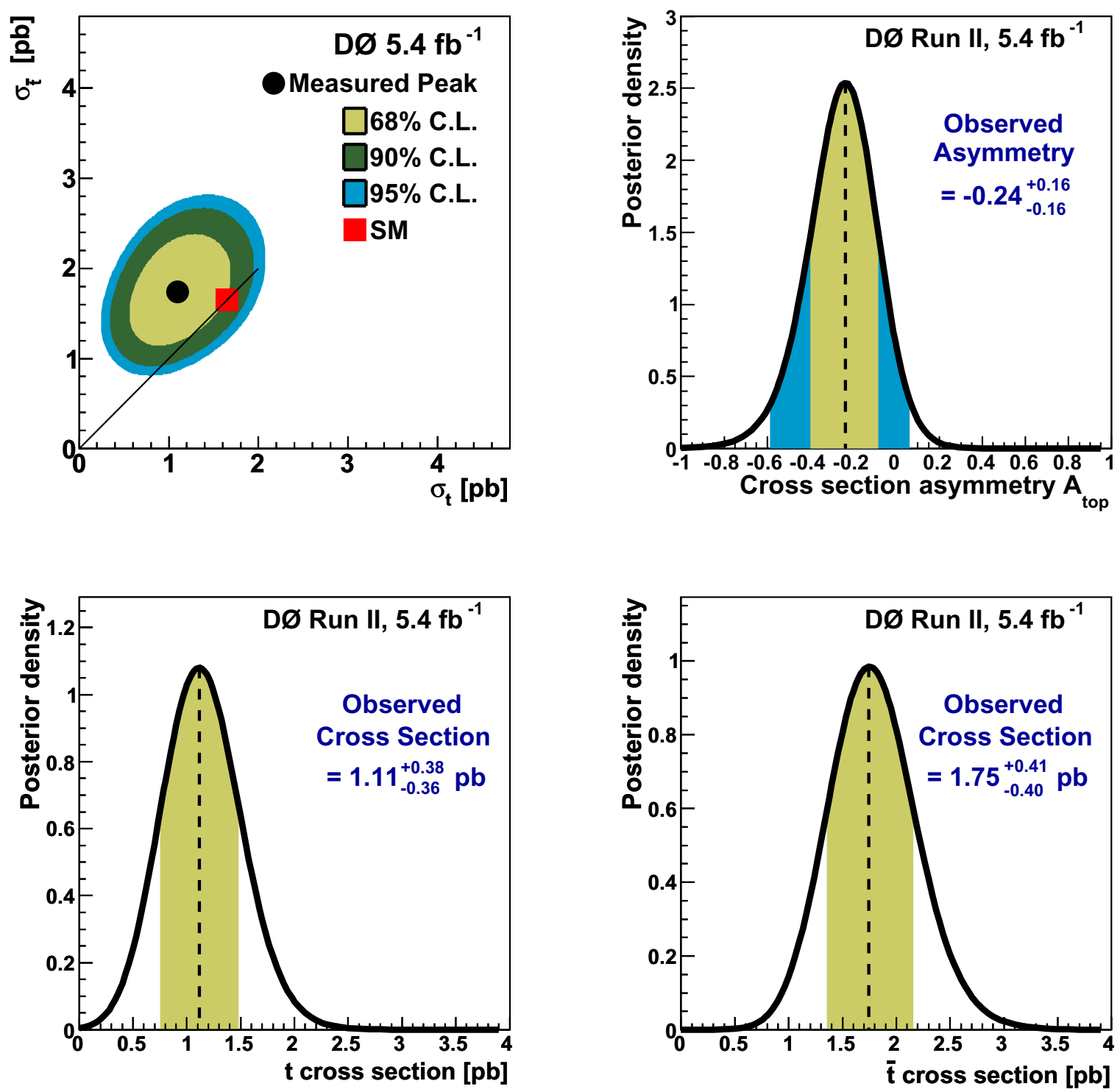

Figure 11.6: Observed posterior density distributions and measurements of top and anti-top cross sections in the $s+t$-channel. The top left plot shows the $2 d$ posterior of top and antitop production cross sections; top right plot shows the posterior of the top-antitop production cross section assymmetry $A_{t o p}$; bottom left plot shows the posterior of the top production cross section; bottom right plot shows the posterior of the antitop production cross section. The top (antitop) cross section are extracted by integrating over the antitop (top) axis respectively from the $2 \mathrm{~d}$ posterior. 
h2
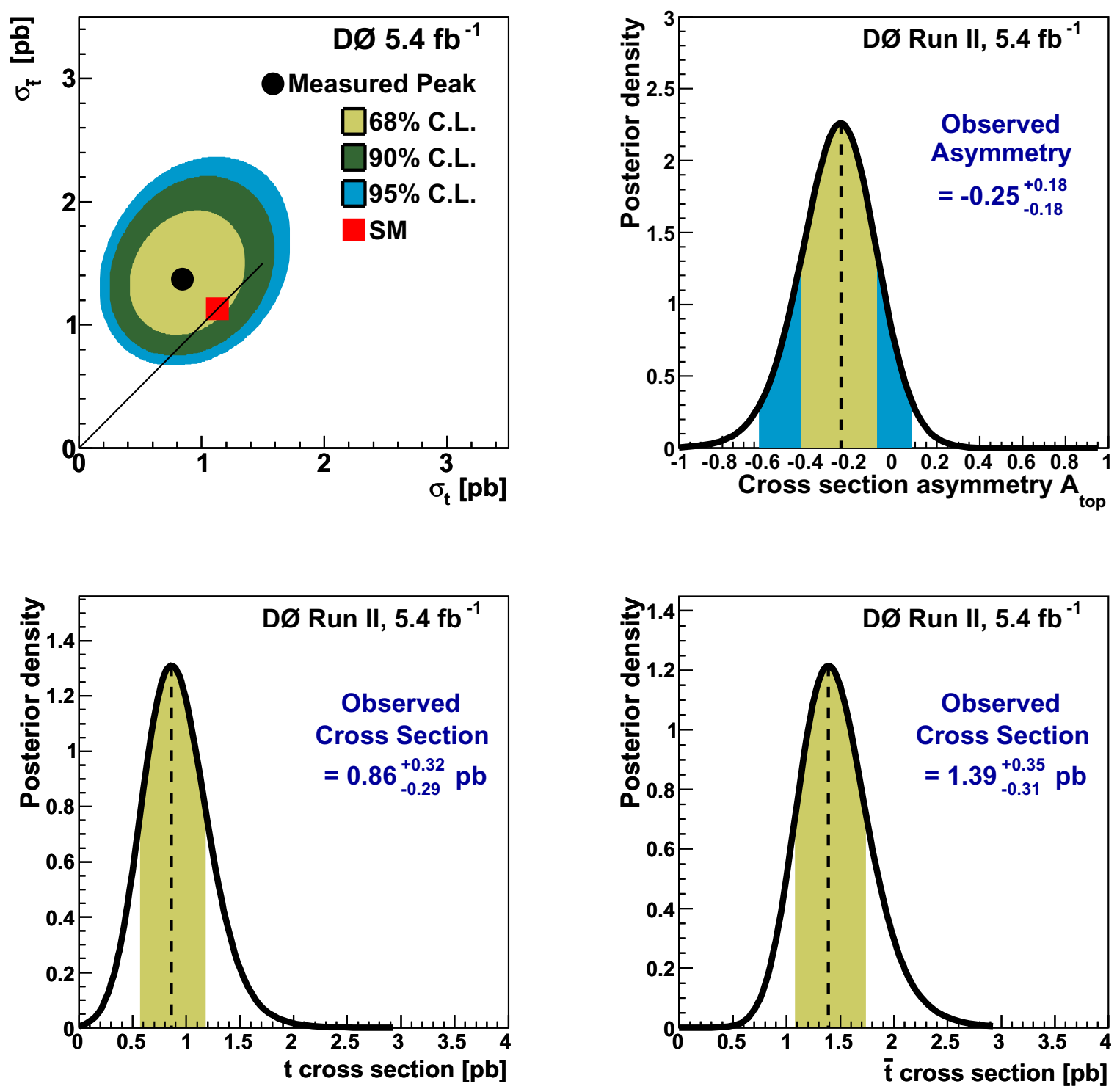

Figure 11.7: Observed posterior density distributions and measurements of top and anti-top cross sections in the $t$-channel. The top left plot shows the $2 d$ posterior of top and antitop production cross sections; top right plot shows the posterior of the top-antitop production cross section assymmetry $A_{t o p}$; bottom left plot shows the posterior of the top production cross section; bottom right plot shows the posterior of the antitop production cross section. The top (antitop) cross section are extracted by integrating over the antitop (top) axis respectively from the $2 \mathrm{~d}$ posterior. 
h2
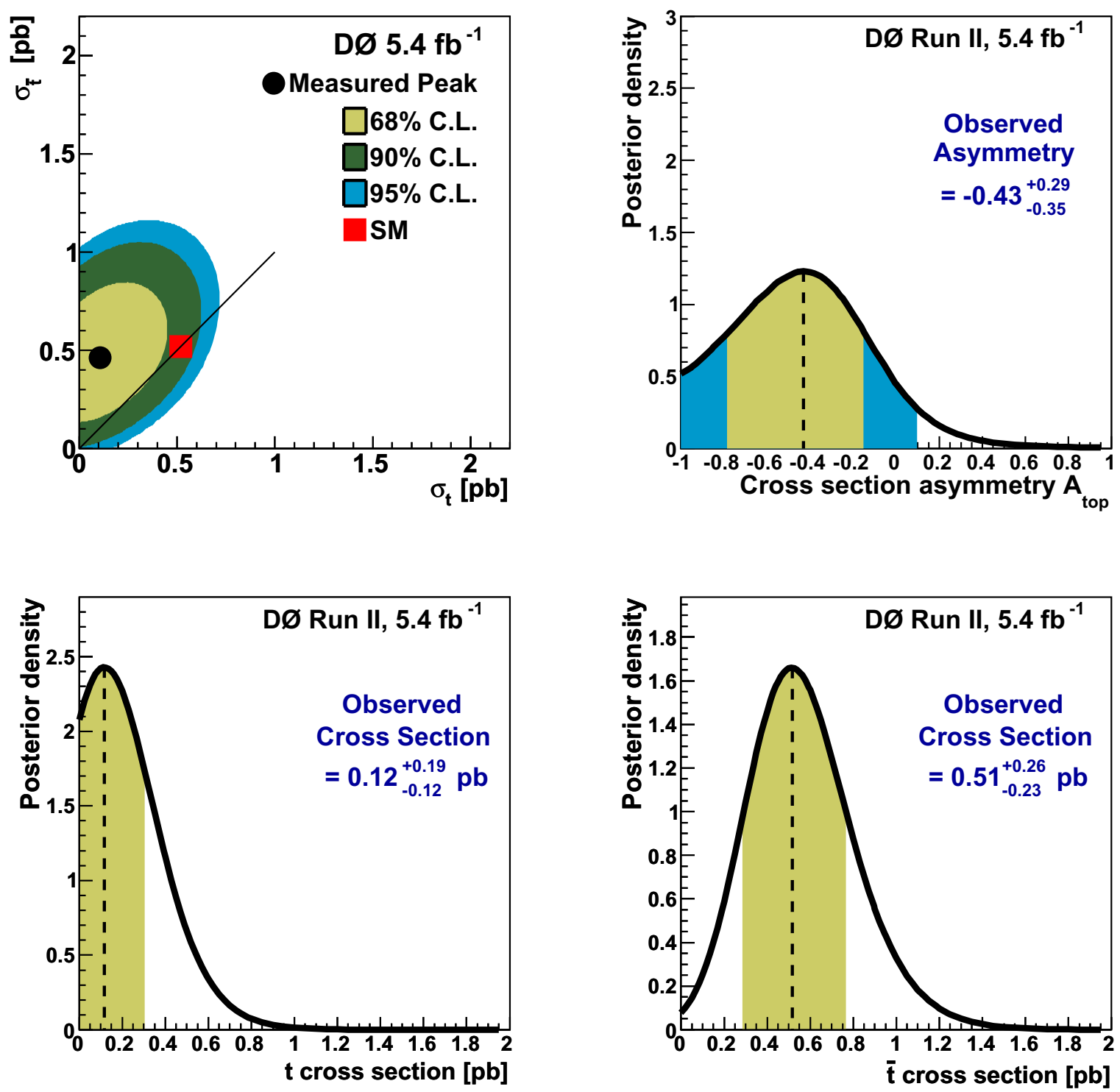

Figure 11.8: Observed posterior density distributions and measurements of top and anti-top cross sections in the $s$-channel. The top left plot shows the $2 d$ posterior of top and antitop production cross sections; top right plot shows the posterior of the top-antitop production cross section assymmetry $A_{t o p}$; bottom left plot shows the posterior of the top production cross section; bottom right plot shows the posterior of the antitop production cross section. The top (antitop) cross section are extracted by integrating over the antitop (top) axis respectively from the $2 \mathrm{~d}$ posterior. 
The observed results for CP asymmetry in $s, t, s+t$ channels for different systematics are summarized in Table 11.2.

Table 11.2: Observed results for $\mathrm{CP}$ asymmetry in $\mathrm{s}, \mathrm{t}, \mathrm{s}+\mathrm{t}$ channels.

\begin{tabular}{c|c} 
Discriminant & CP Asymmetry \\
\hline$t b$ & $-0.43_{-0.35}^{+0.29}$ \\
$t q b$ & $-0.25_{-0.18}^{+0.18}$ \\
$t b+t q b$ & $-0.24_{-0.16}^{+0.18}$ \\
\hline
\end{tabular}




\section{Chapter 12}

\section{Results and Conclusions}

In this thesis we present a search for $\mathrm{CP}$ violation using the single top quark final state with the $D \varnothing$ detector at the Fermilab Tevatron. We perform the search separately in the $s$-, $t$ - and $s+t$-channel using $5.4 \mathrm{fb}^{-1}$ of data. This analysis is an extension of the single top cross section measurement, and is the first analysis searching for $\mathrm{CP}$ violation in single top quark production.

The measured asymmetry $\mathcal{A}$ for $s-, t$ - and $s+t$-channel are $-0.43_{-0.35}^{+0.29},-0.25_{-0.18}^{+0.18}$ and $-0.24_{-0.16}^{+0.16}$ respectively. 
APPENDICIES 


\section{Appendix A}

\section{Plots After Splitting by the}

\section{Lepton Charge}

Figures A.2 to A.24 show various kinematic distributions in the tagged final samples after being splitted by the lepton charge. The plots are shown on the left for the positive samples and on the right for the negative samples. The legends are only shown in the first pair of plots (Fig. A.2). All other plots assume the same legend. The signal and background distributions use the color scheme illustrated in Figure A.1. 


\section{Key for Plots}

- Data

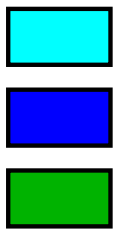

Signal: $t b$

Signal: $t q b$

$W b \bar{b}+$ jets

$W c \bar{c}+$ jets

$\square W+$ light jets

$\square Z b \bar{b}+$ jets

$\square Z c \bar{c}+\mathrm{jets}$

$\square Z+$ light jets

$W W+W Z+Z Z$

$t \bar{t} \rightarrow l l$

$t \bar{t} \rightarrow l+\mathrm{jets}$

Multijets

Figure A.1: Illustration of the color scheme used in plots of signals and backgrounds in the single top analyses. 

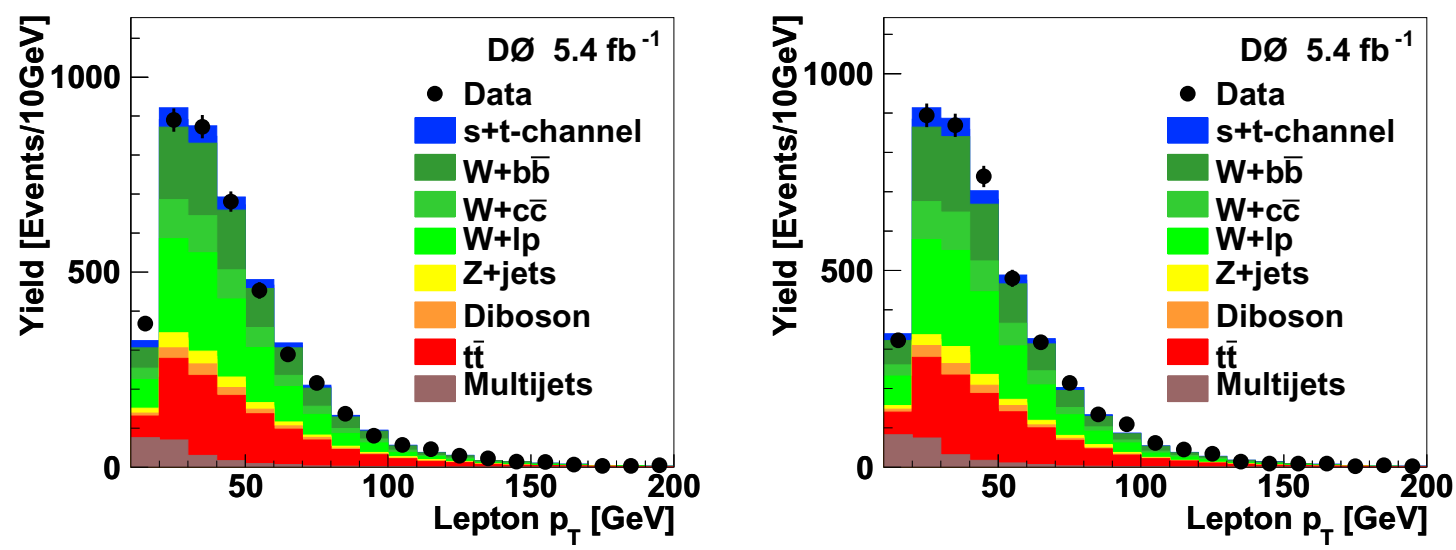

Figure A.2: The transverse momentum of the lepton in the electron+muon channel for positive (left) and negative (right) samples.
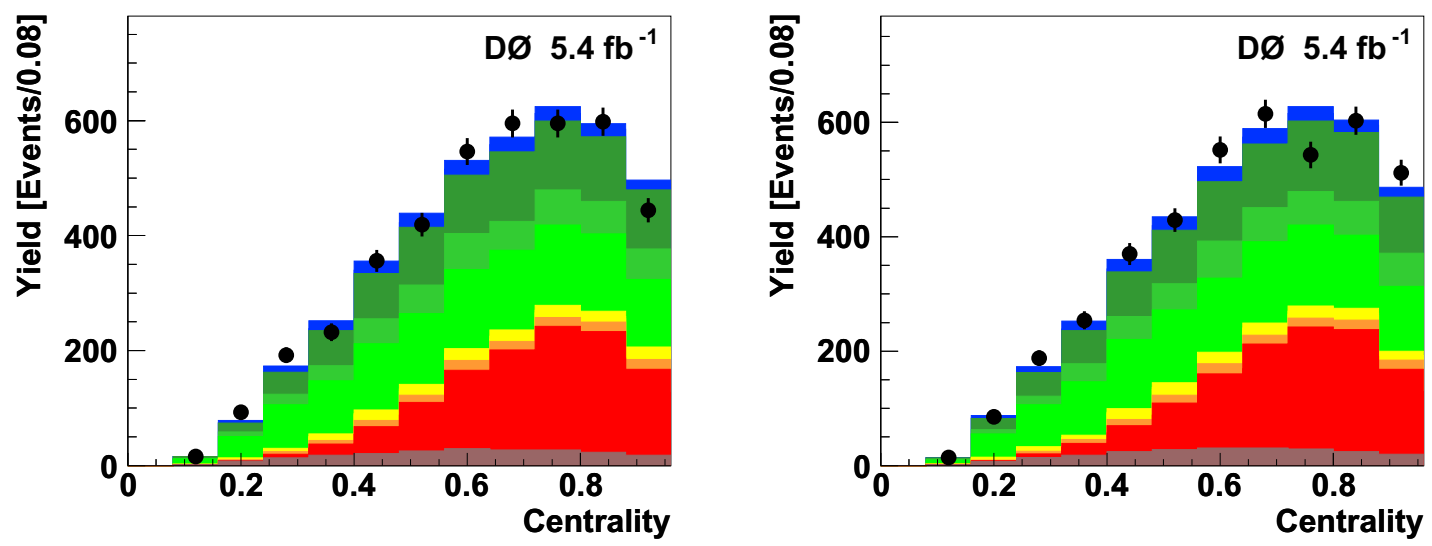

Figure A.3: The Centrality for All Jets in the electron+muon channel for positive (left) and negative (right) samples. 

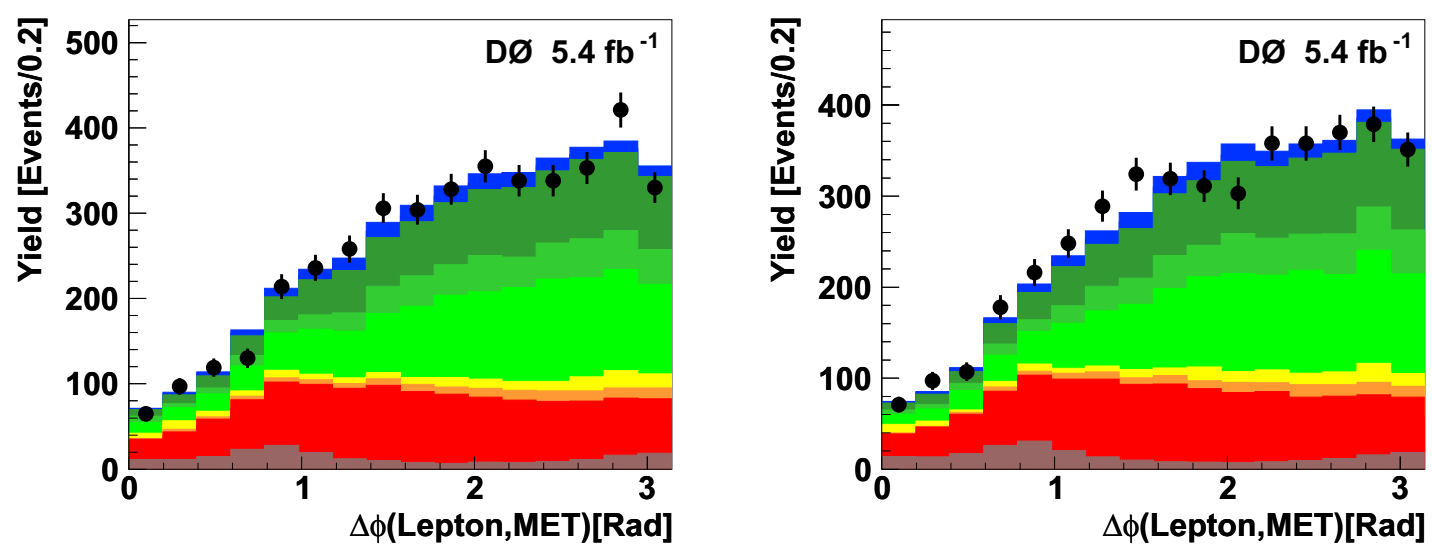

Figure A.4: The Delta Phi between Lepton MET in the electron+muon channel for positive (left) and negative (right) samples.
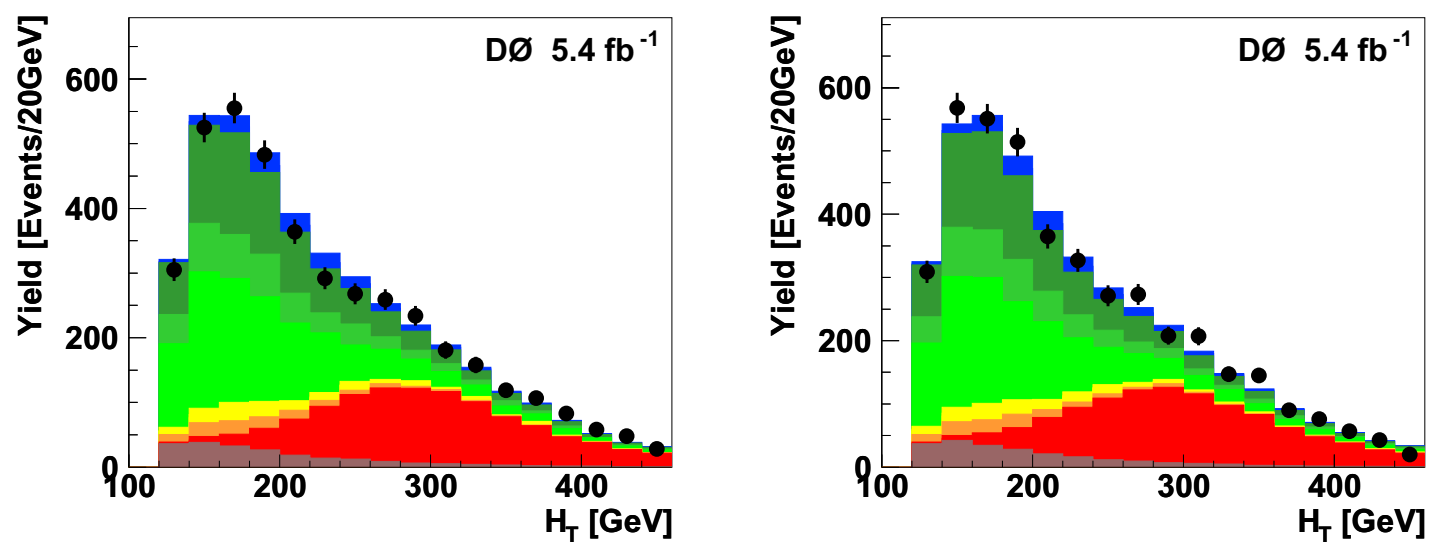

Figure A.5: The HT in the electron+muon channel for positive (left) and negative (right) samples. 

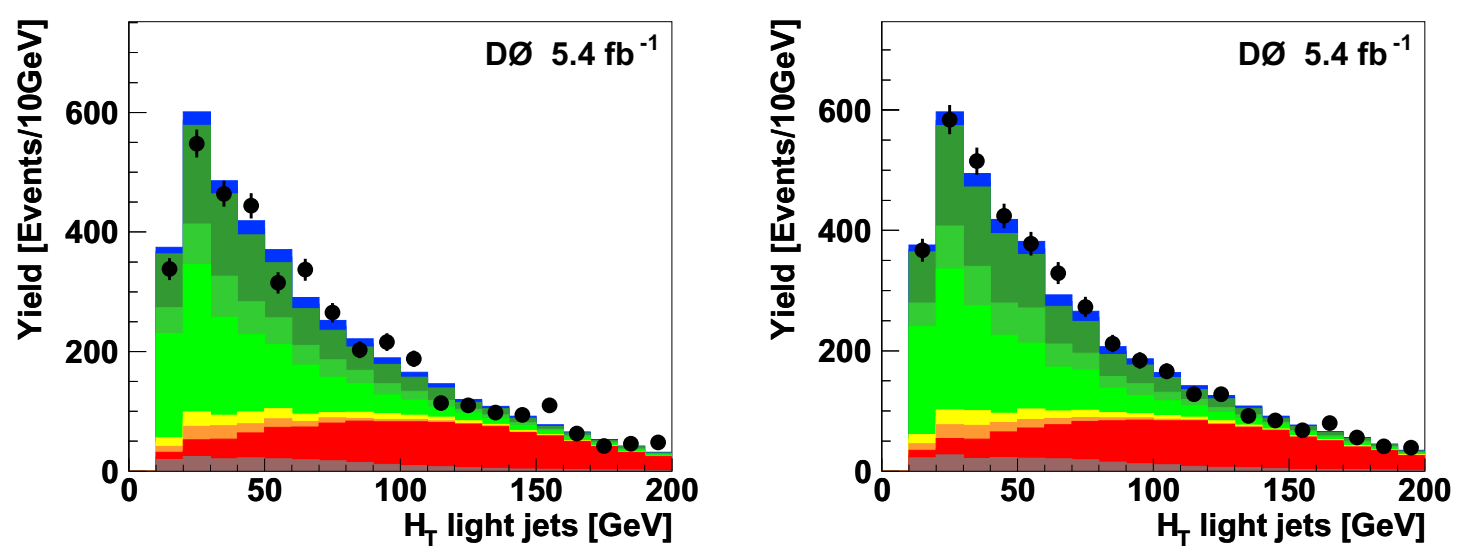

Figure A.6: The HT-AllJets-MinusBTaggedJet in the electron+muon channel for positive (left) and negative (right) samples.
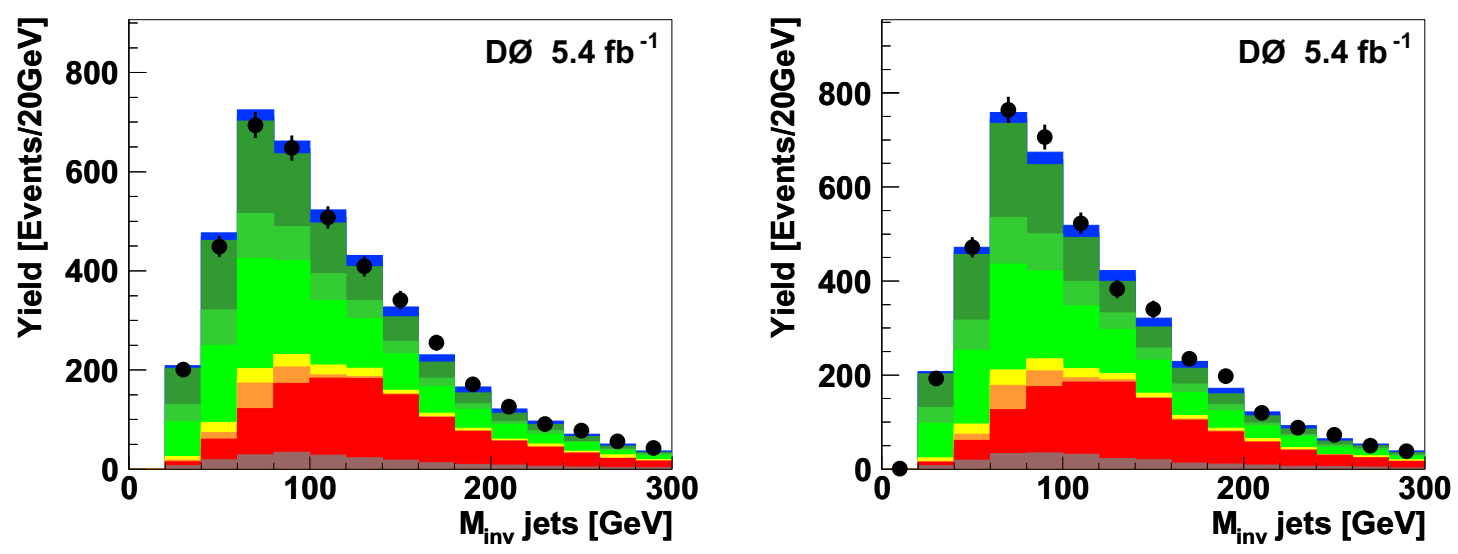

Figure A.7: The Invariant Mass for the two leading jets in the electron+muon channel for positive (left) and negative (right) samples. 

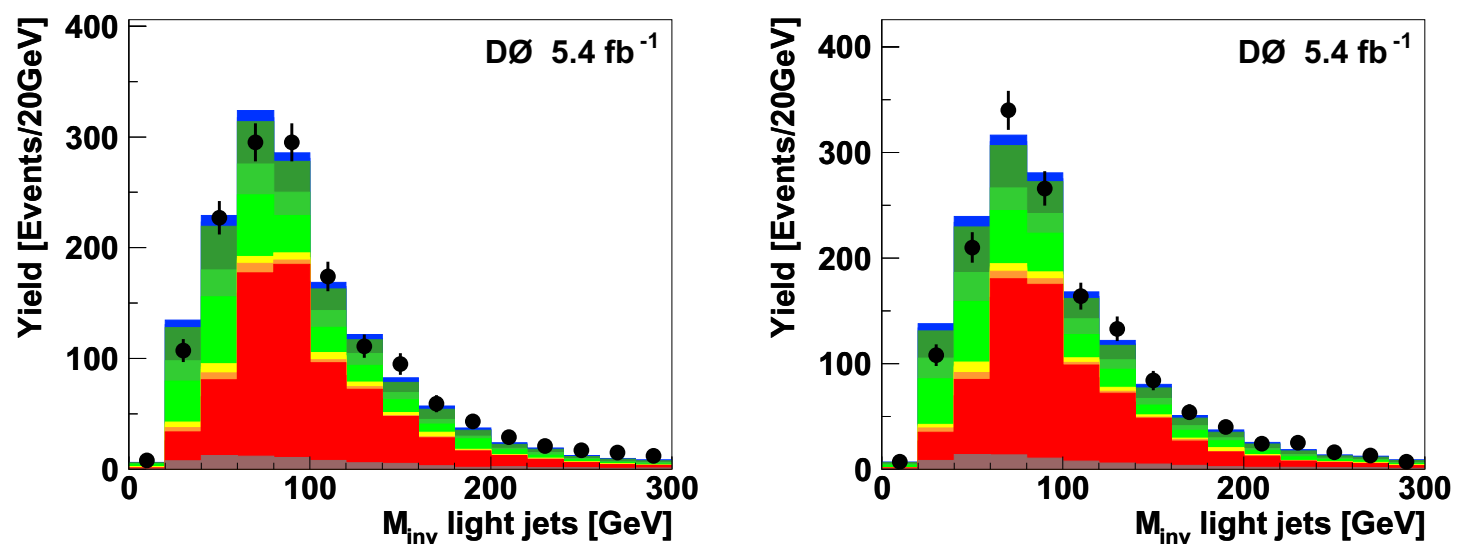

Figure A.8: The InvariantMass-LightQuarkJets1-2 in the electron+muon channel for positive (left) and negative (right) samples.
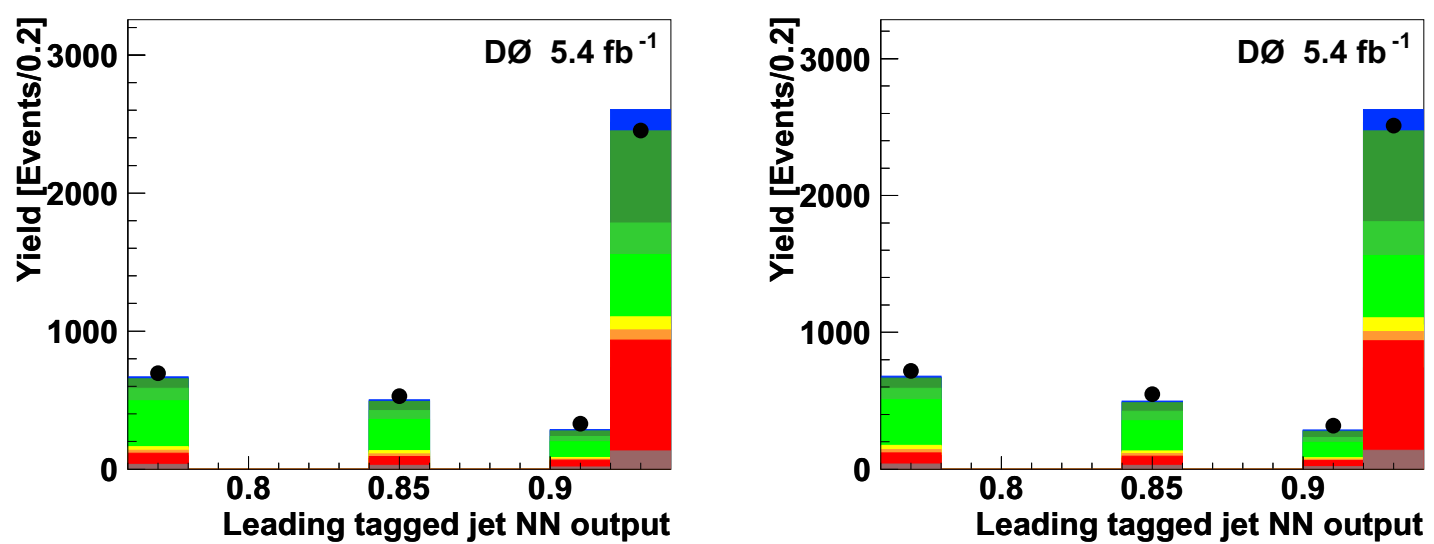

Figure A.9: The Leading b Tagged Jet in the electron+muon channel for positive (left) and negative (right) samples. 

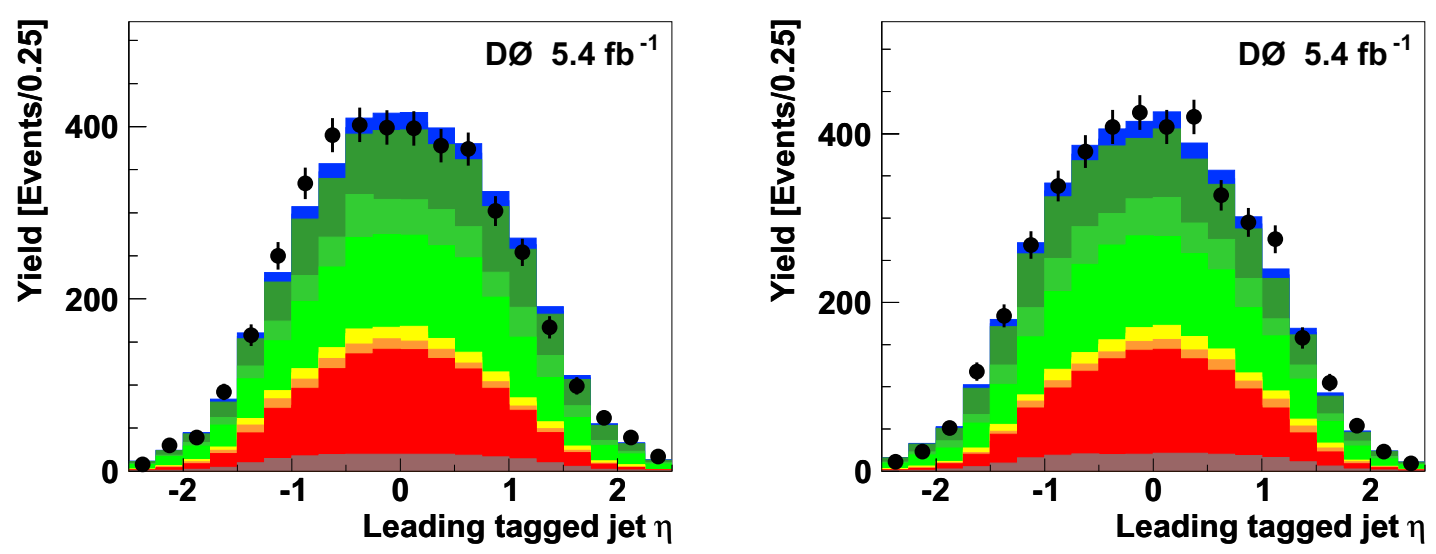

Figure A.10: The LeadingBTaggedJetEta in the electron+muon channel for positive (left) and negative (right) samples.
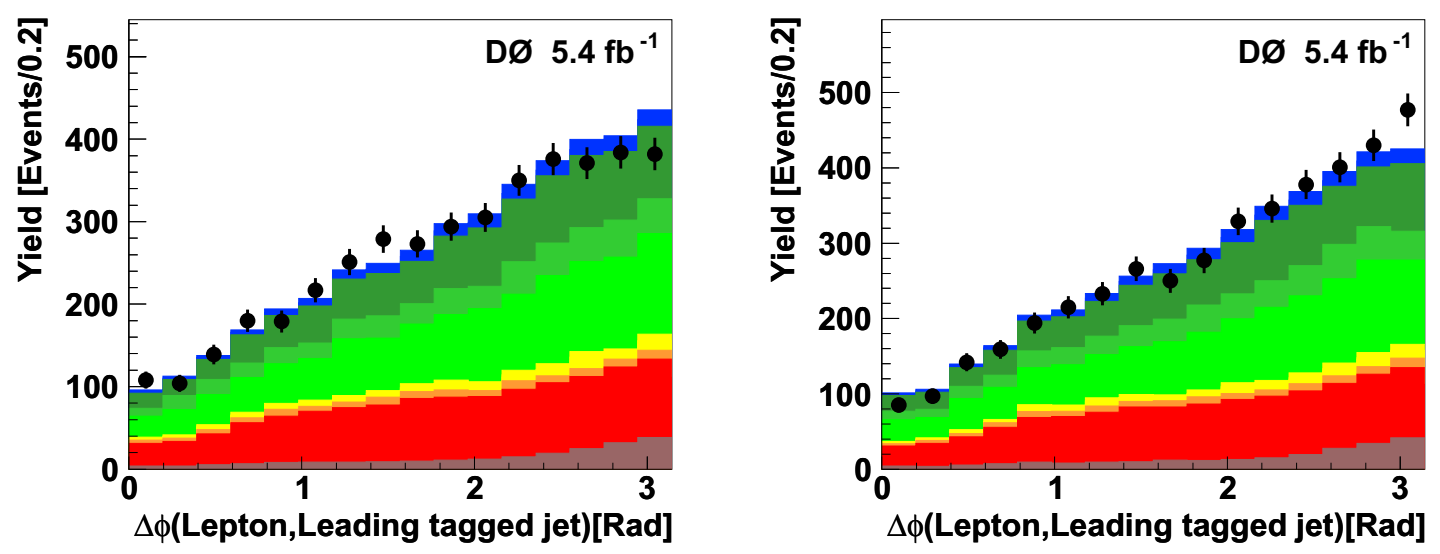

Figure A.11: The LeadingBTaggedJetLeptonDeltaPhi in the electron+muon channel for positive (left) and negative (right) samples. 

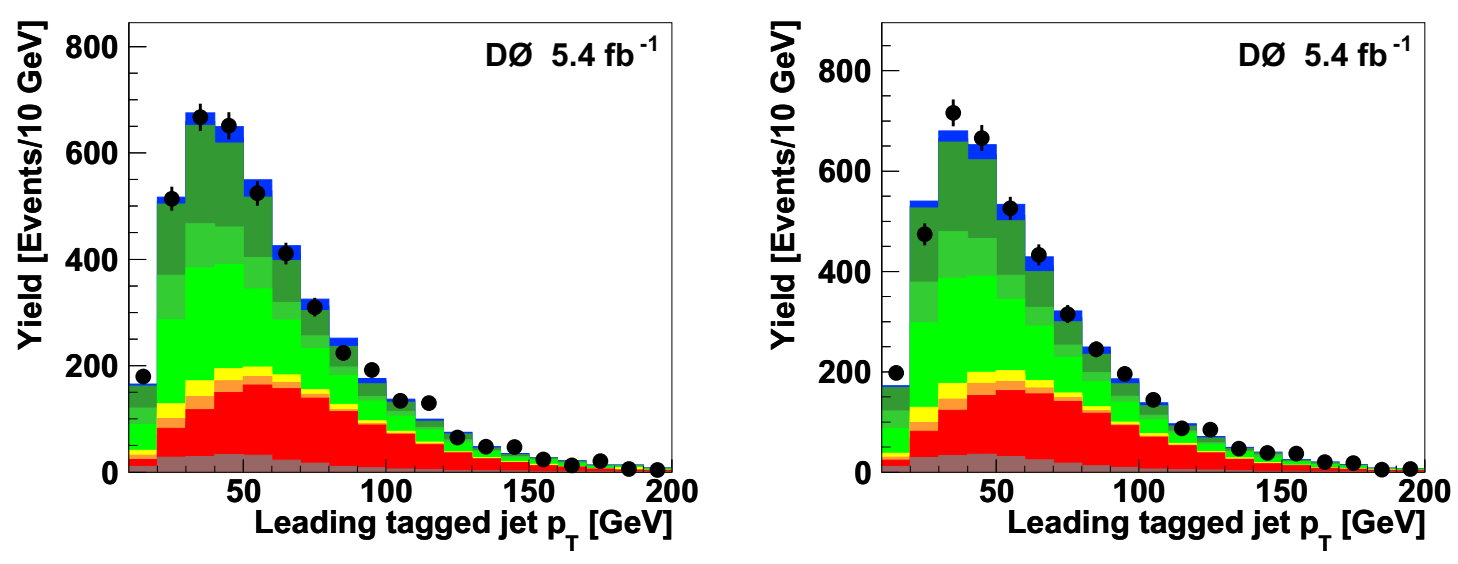

Figure A.12: The LeadingBTaggedJetPt in the electron+muon channel for positive (left) and negative (right) samples.
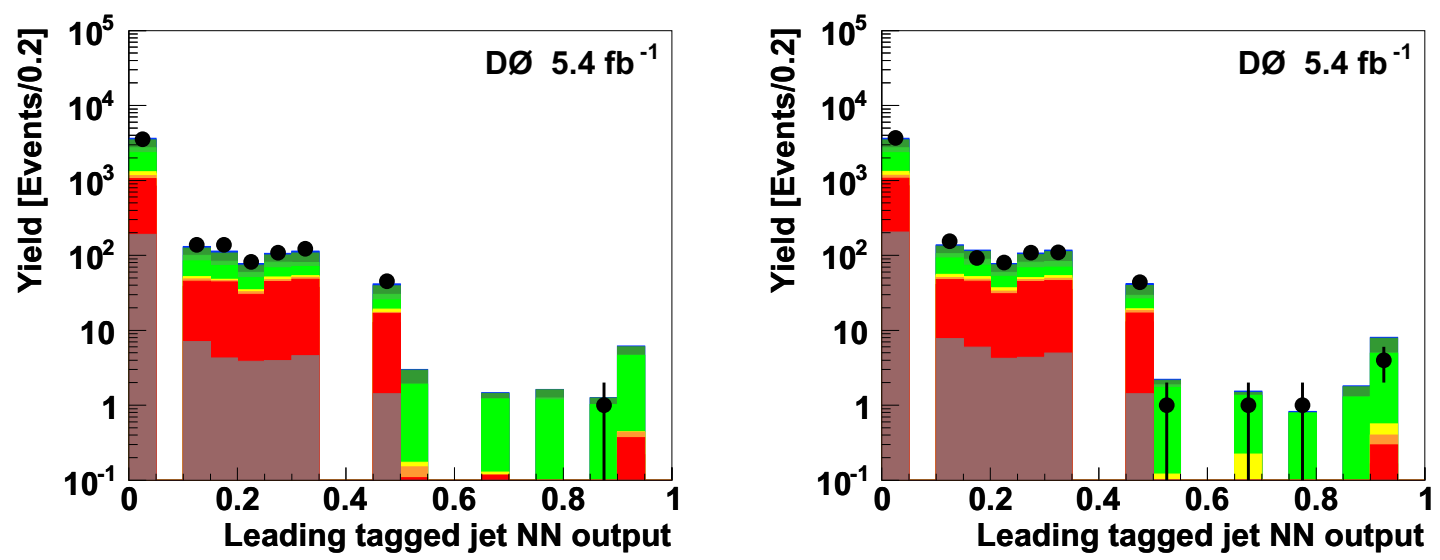

Figure A.13: The LeadingLightQuarkJetBTagNN in the electron+muon channel for positive (left) and negative (right) samples. 

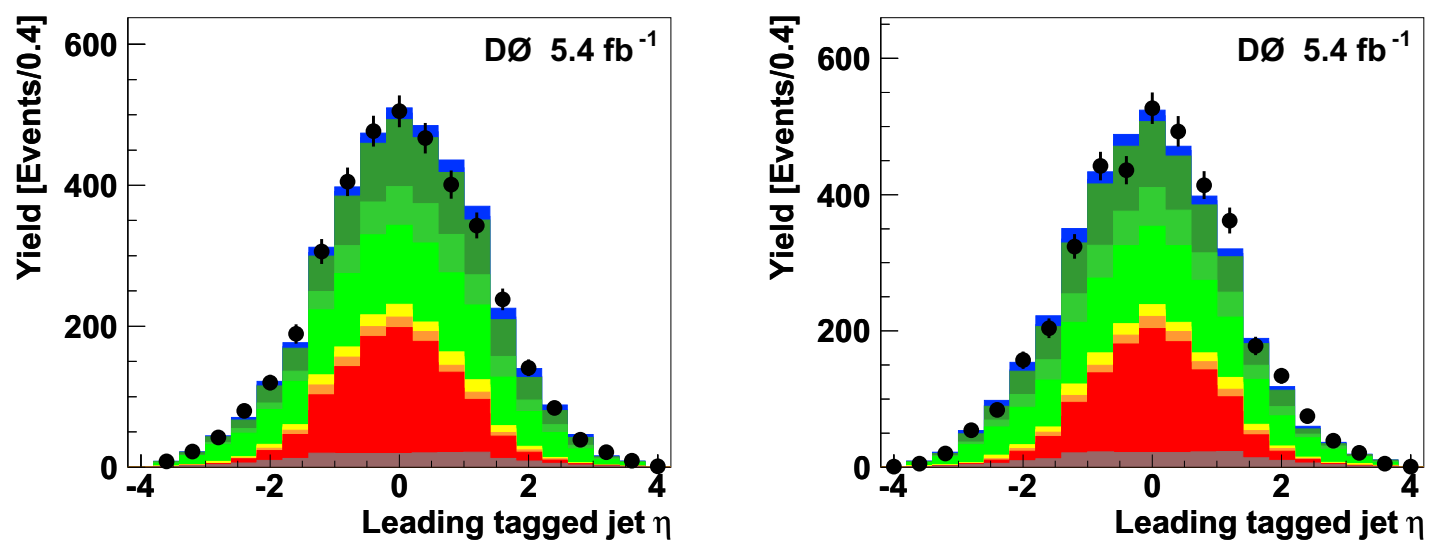

Figure A.14: The LeadingLightQuarkJetEta in the electron+muon channel for positive (left) and negative (right) samples.
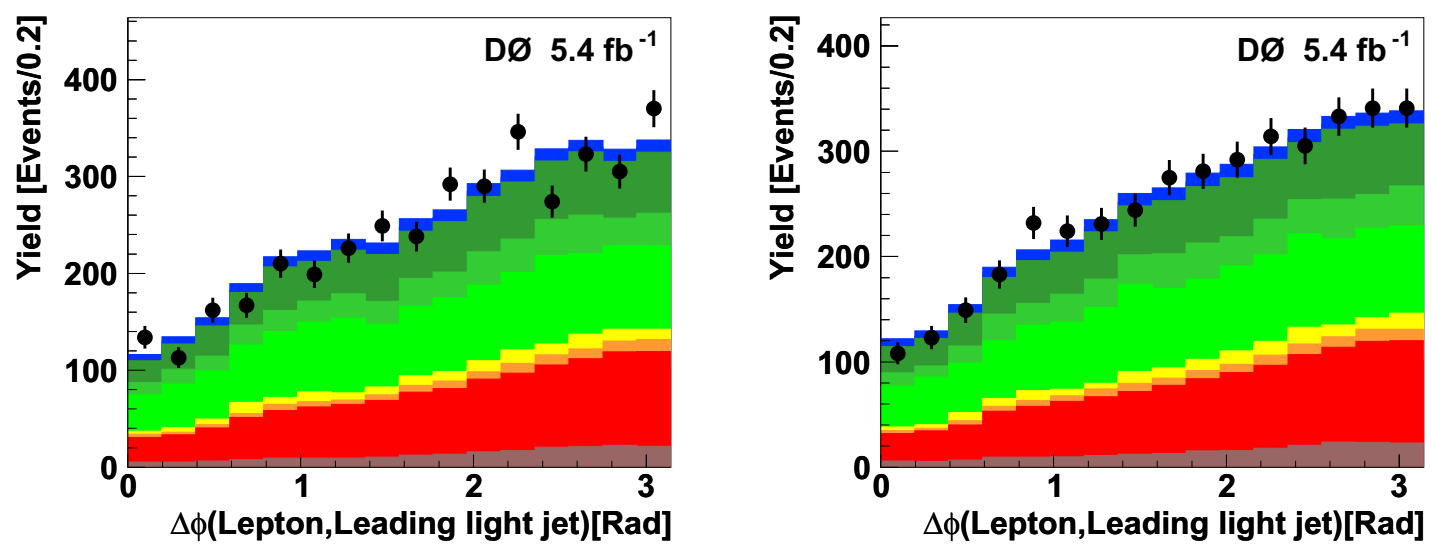

Figure A.15: The LeadingLightQuarkJetLeptonDeltaPhi in the electron+muon channel for positive (left) and negative (right) samples. 

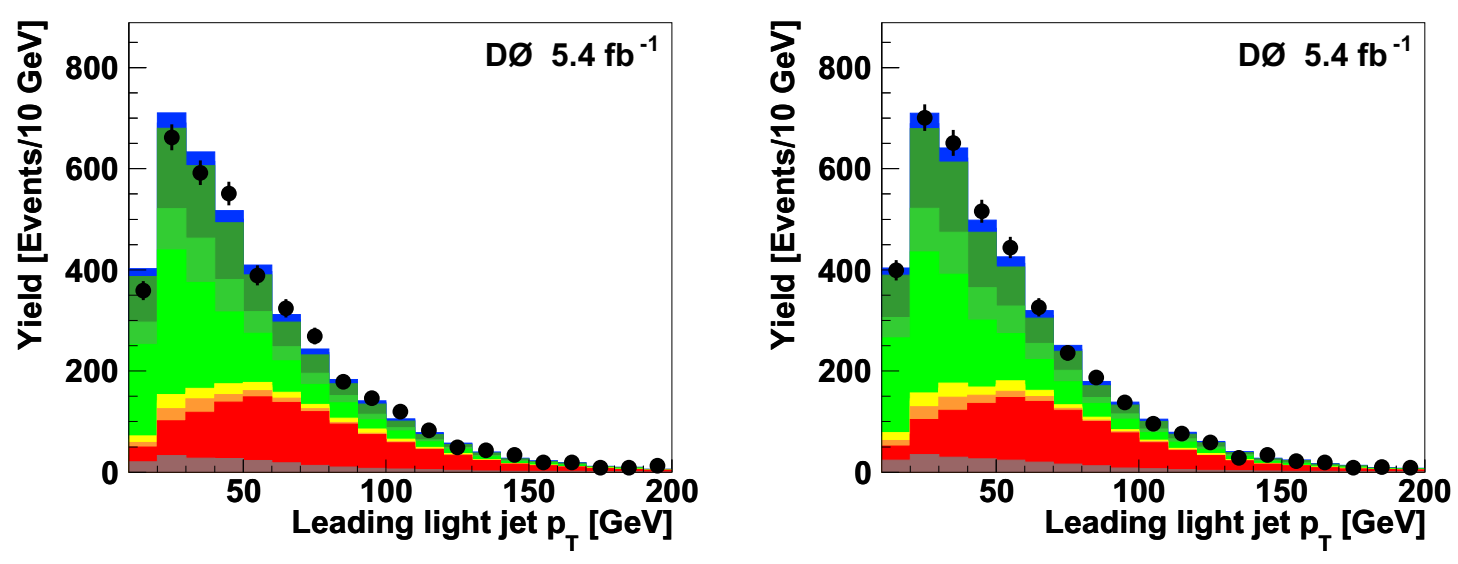

Figure A.16: The LeadingLightQuarkJetPt in the electron+muon channel for positive (left) and negative (right) samples.
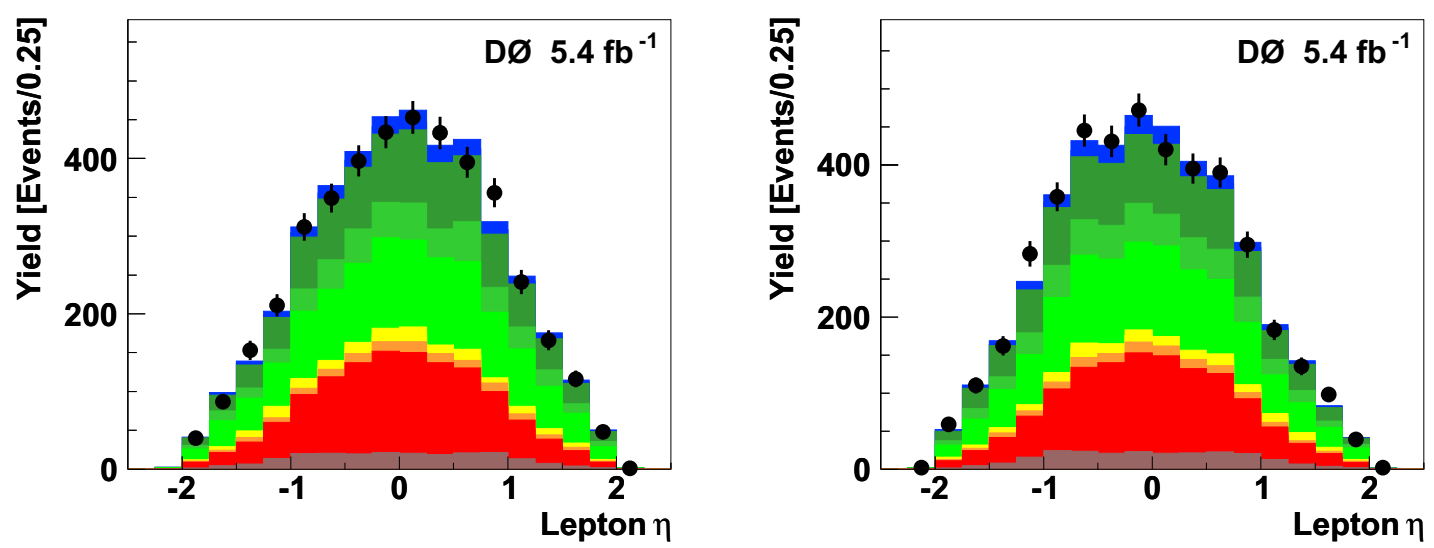

Figure A.17: The LeptonEta in the electron+muon channel for positive (left) and negative (right) samples. 

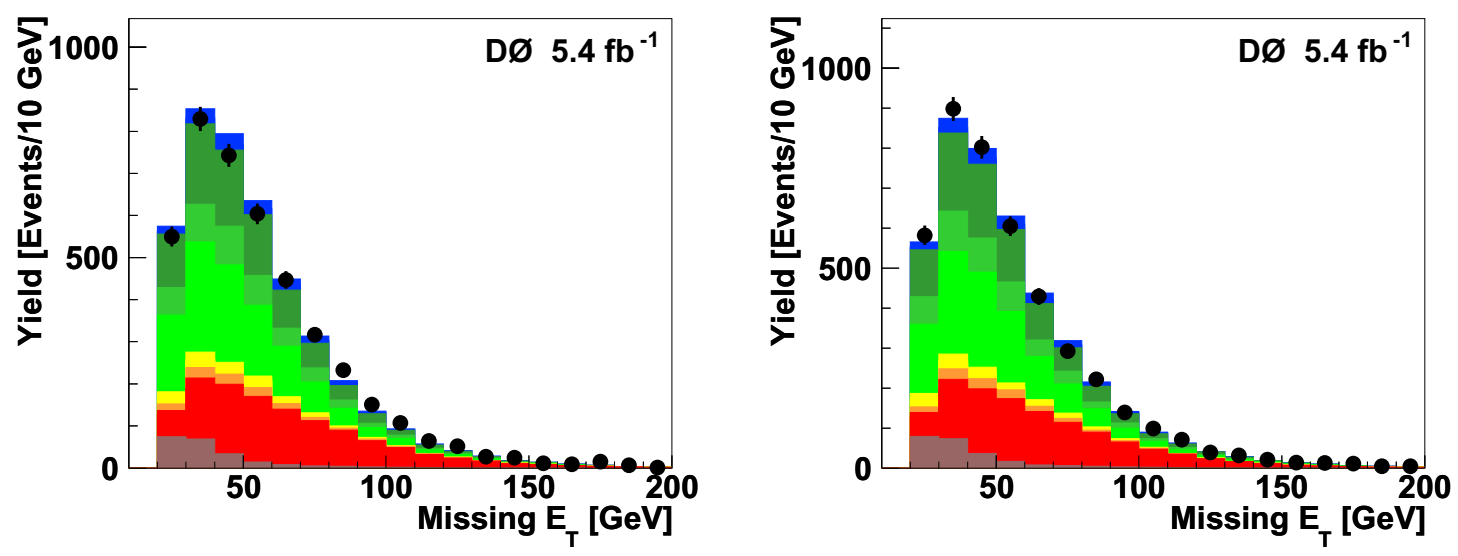

Figure A.18: The METPt in the electron+muon channel for positive (left) and negative (right) samples.
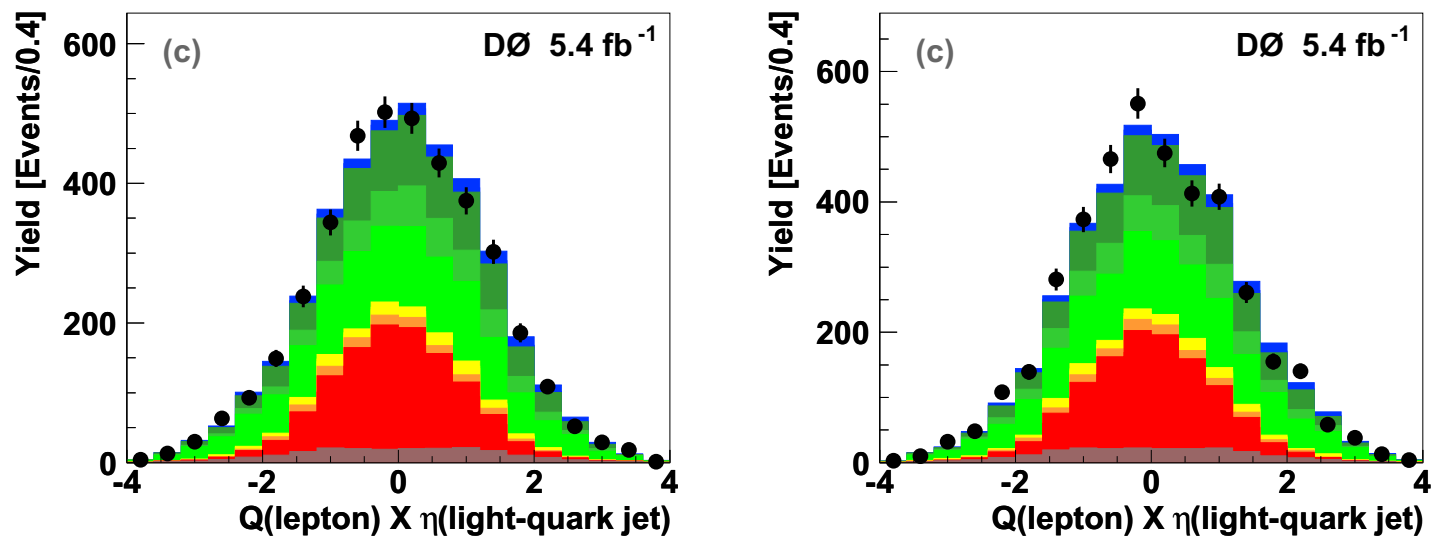

Figure A.19: The QTimesEta in the electron+muon channel for positive (left) and negative (right) samples. 

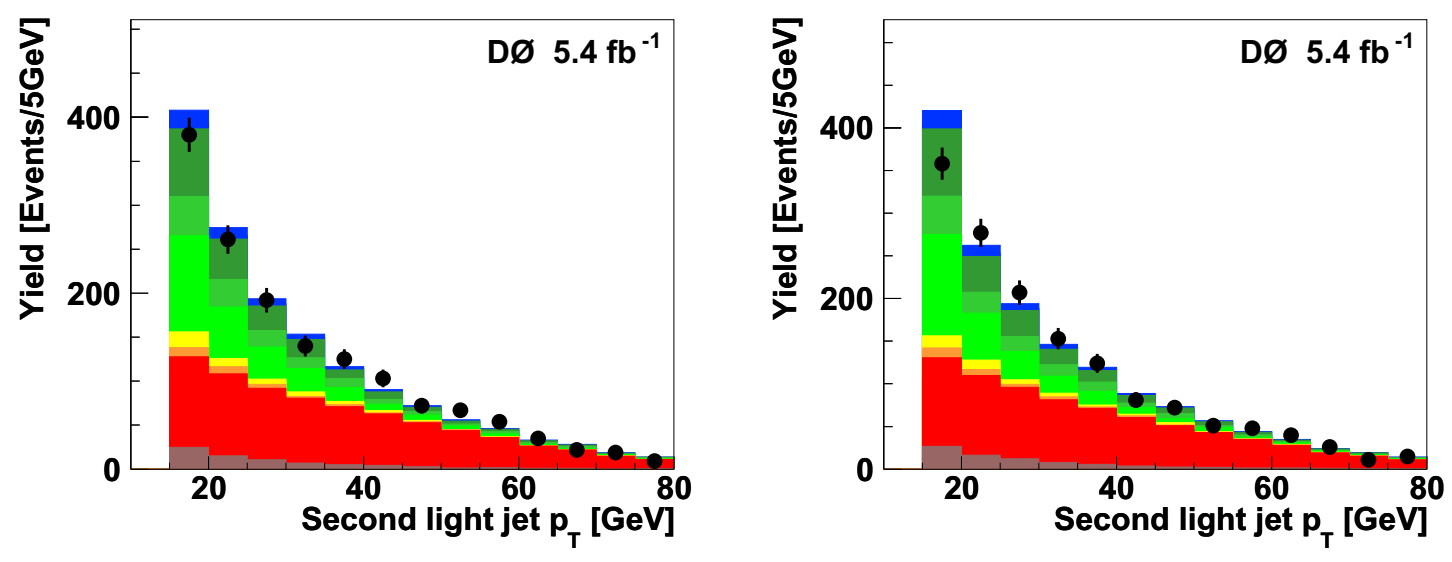

Figure A.20: The SecondLightQuarkJetPt in the electron+muon channel for positive (left) and negative (right) samples.
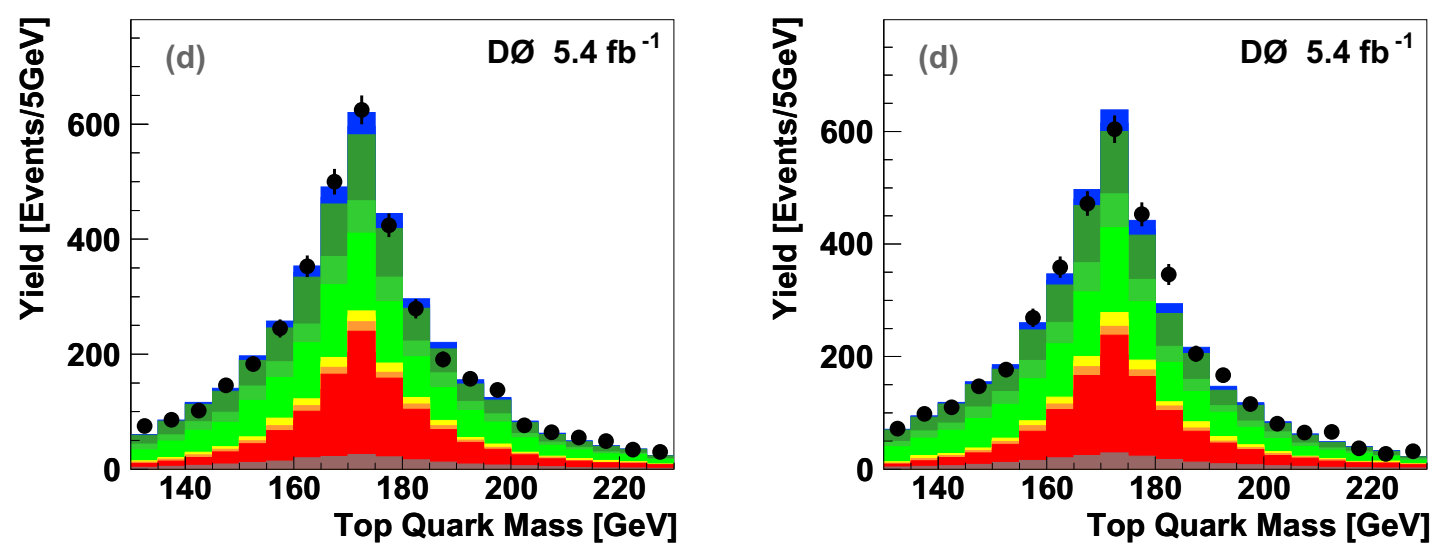

Figure A.21: The SigTopMass in the electron+muon channel for positive (left) and negative (right) samples. 

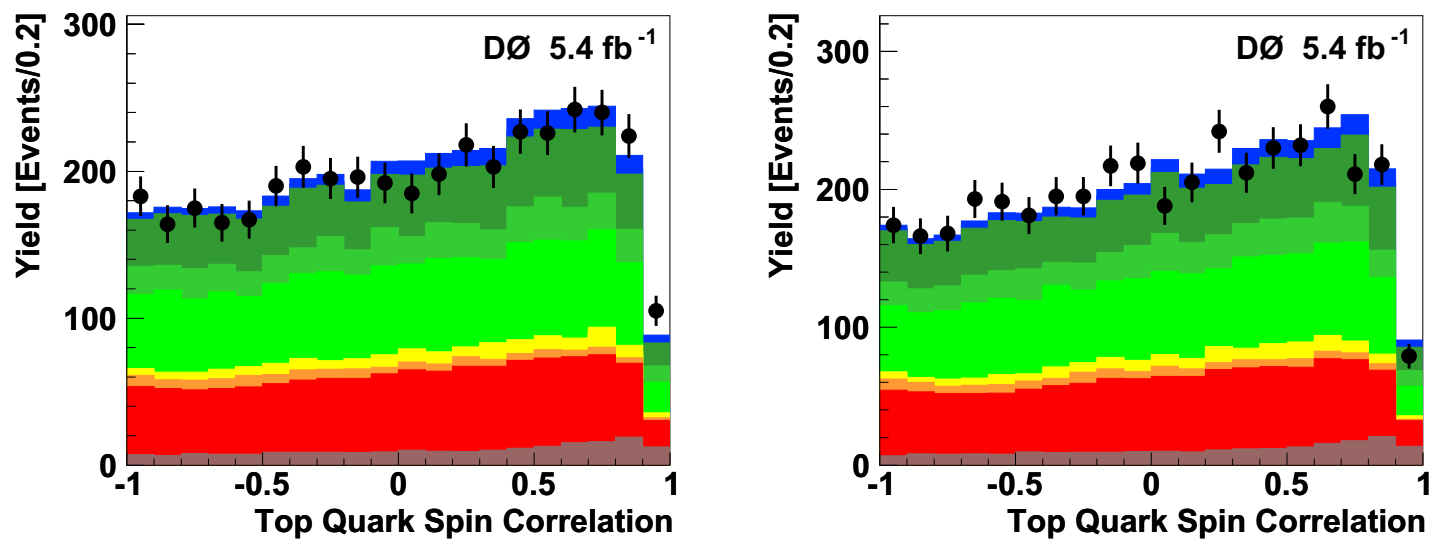

Figure A.22: The SpinCorr in the electron+muon channel for positive (left) and negative (right) samples.
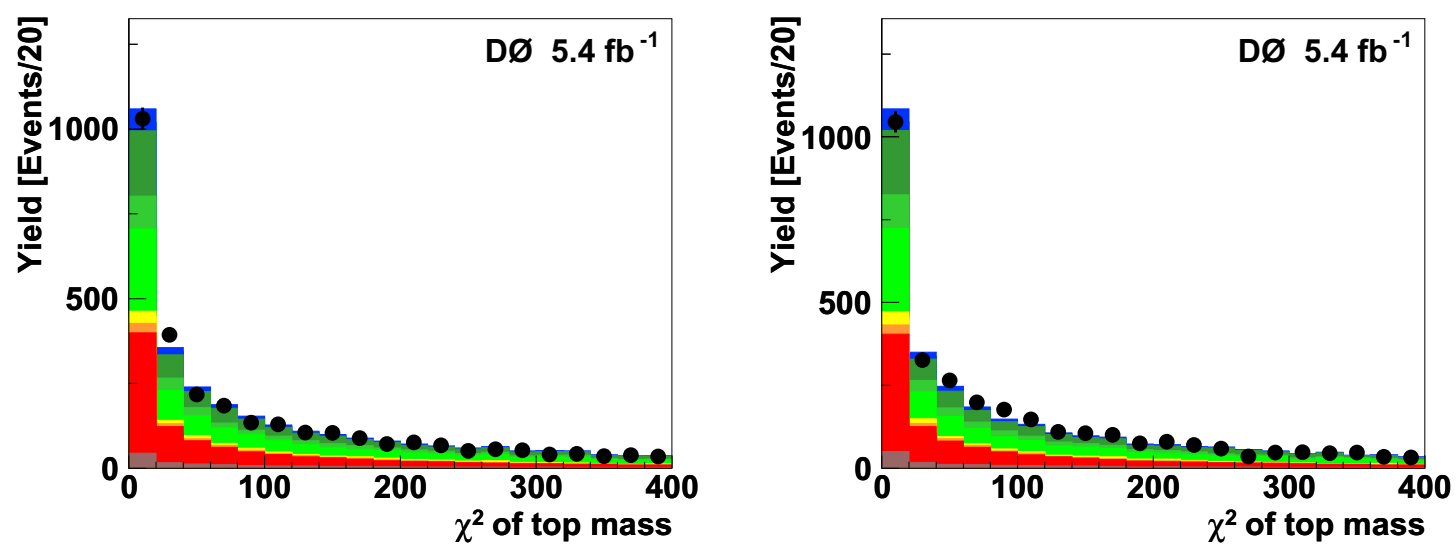

Figure A.23: The TopMassMinChiSqr in the electron+muon channel for positive (left) and negative (right) samples. 

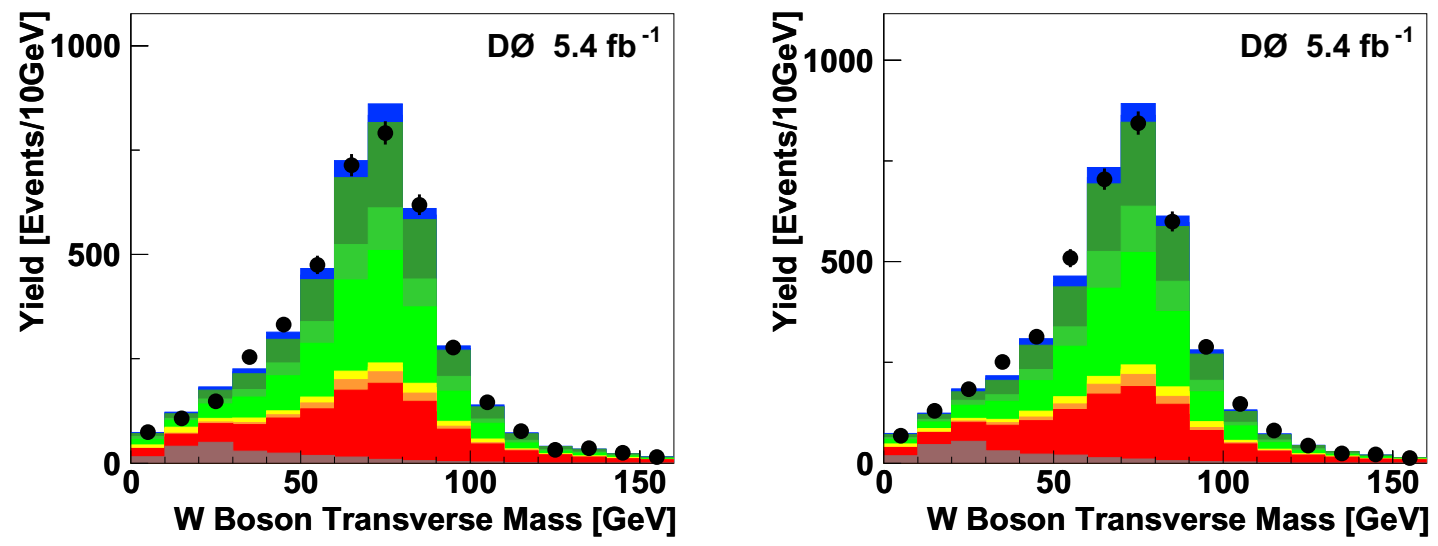

Figure A.24: The WT in the electron+muon channel for positive (left) and negative (right) samples. 


\section{Appendix B}

\section{SAM Definitions of Dataset Used in This Analysis}

The dataset is obtained from the MUinclusive and EMinclusive common sample group (CSG) skims:

- CSG_CAF_skim_PASS3_p18.13.01 for RunIIa

- CSG_CAF_skim_PASS2_p21.10.00 for Run IIb1

- CSG_CAF_skim_PASS4_p21.10.00_p20.12.00-04 and CSG_CAF_skim_PASS4_p21.12.00_p20.12.05_allfix for RunIIb2

and the following VJets definitions

- VJets_EM/MUinclusive_Moriond09_RunIIa_v1 for Run IIa 
- VJets_EMinclusive_Summer09_extended_RunIIb_v3 for Run IIb electrons

- VJets_MUinclusive_Summer09_extended_allfix_RunIIb for RunIIb muons 


\section{Appendix $\mathrm{C}$}

\section{Plots for discriminants after the}

\section{binning transformation}

Figures C.1 to C.6 show various discriminants distributions in the tagged final samples after the binning transformation applied to the discriminants $[58,30]$. When trying to calculate the cross section, in the high discriminant region there may be some bins in which there are some signal but no background events. To avoid this, we applied a binning transformation to MVA outputs that ensures that there is a minimum amount of effective background events in each bin. We followed the same procedure as described in detail in Appendix D of Ref. [58] and applied the same binning transformation function derived for the BNNComb discriminants in Ref. [30] to both positive and negative charge samples. The signal region with discriminant 
output from 0.8 to 1 are zoomed to show that there are in fact signals in that region. The "Sort" or "Ranked" plots sorted each bin according to their S/B ratios from left to right. 

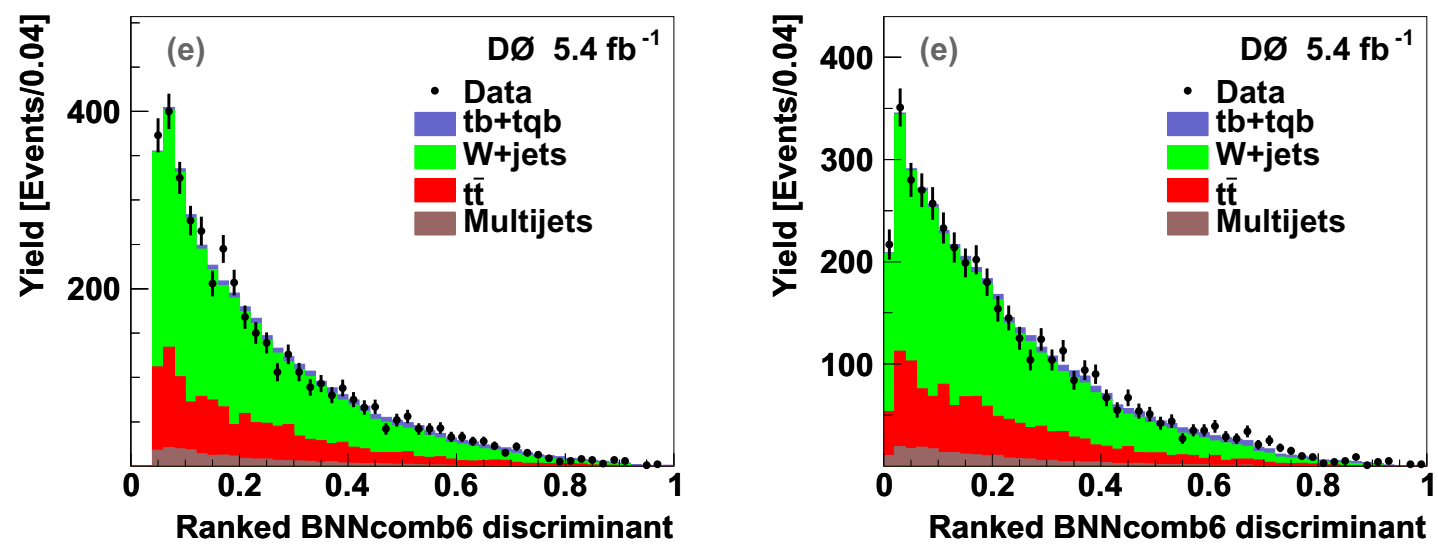

Figure C.1: The $s+t$ discriminant plots in the electron+muon channel for positive (left) and negative (right) events.
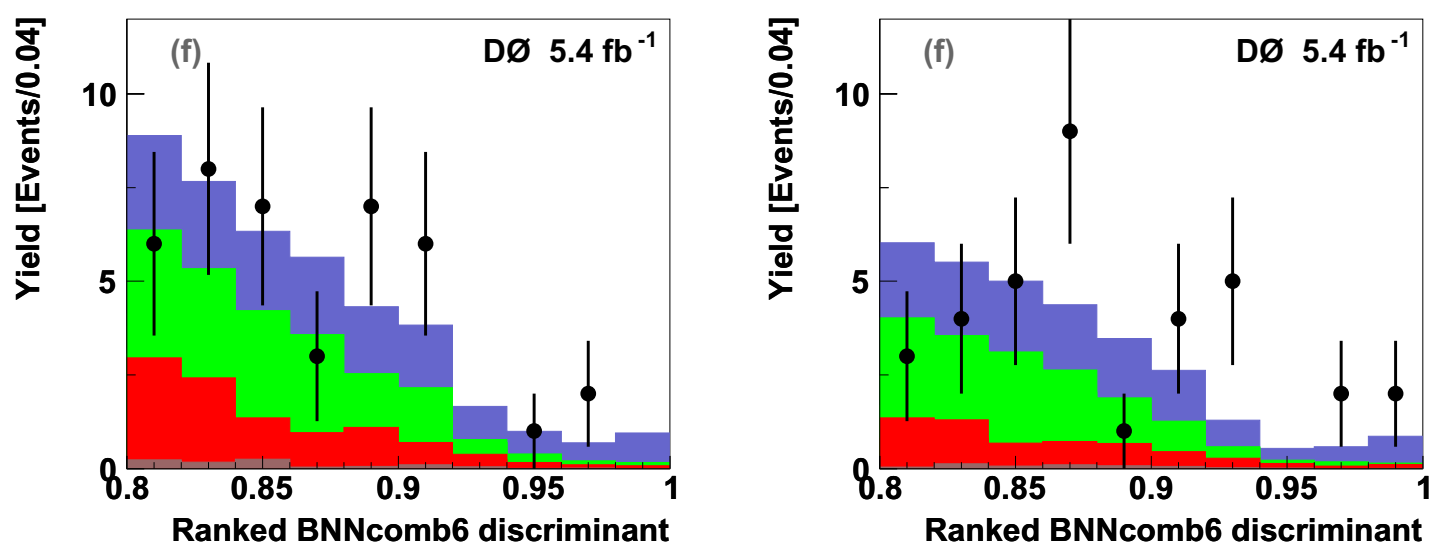

Figure C.2: The BNNcomb6SortZoom plots in the electron+muon channel for positive (left) and negative (right) events. 

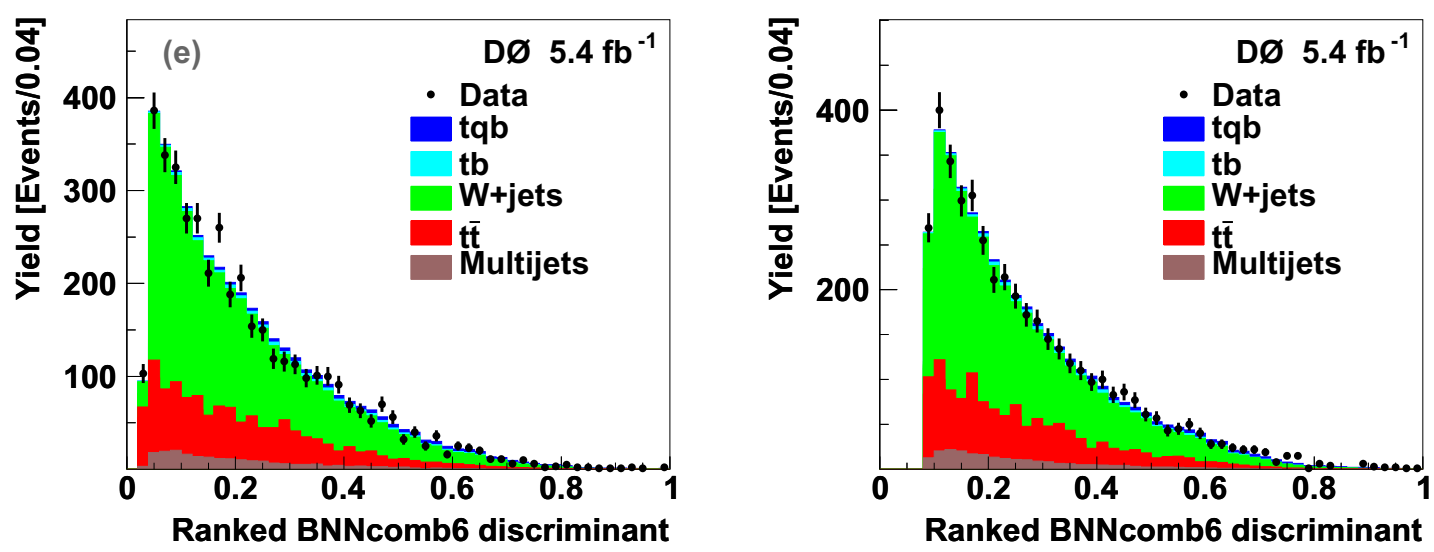

Figure C.3: The $t$ discriminant plots in the electron+muon channel for positive (left) and negative (right) events.
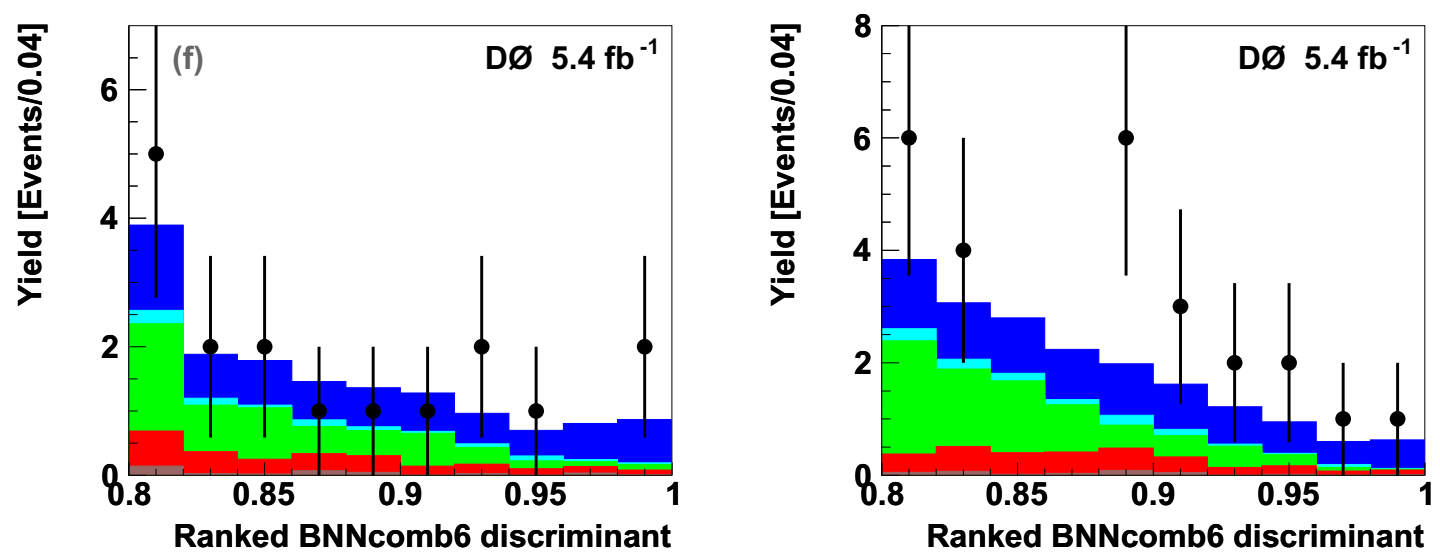

Figure C.4: The BNNcombTSortZoom plots in the electron+muon channel for positive (left) and negative (right) events. 

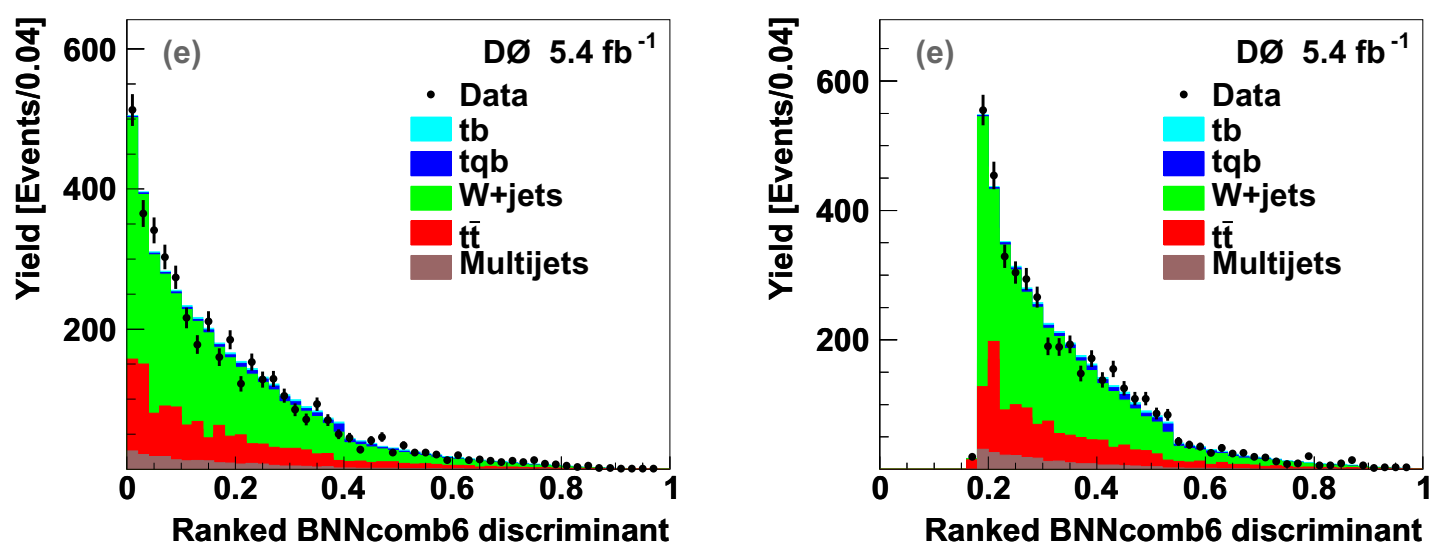

Figure C.5: The $s$ discriminant plots in the electron+muon channel for positive (left) and negative (right) events.
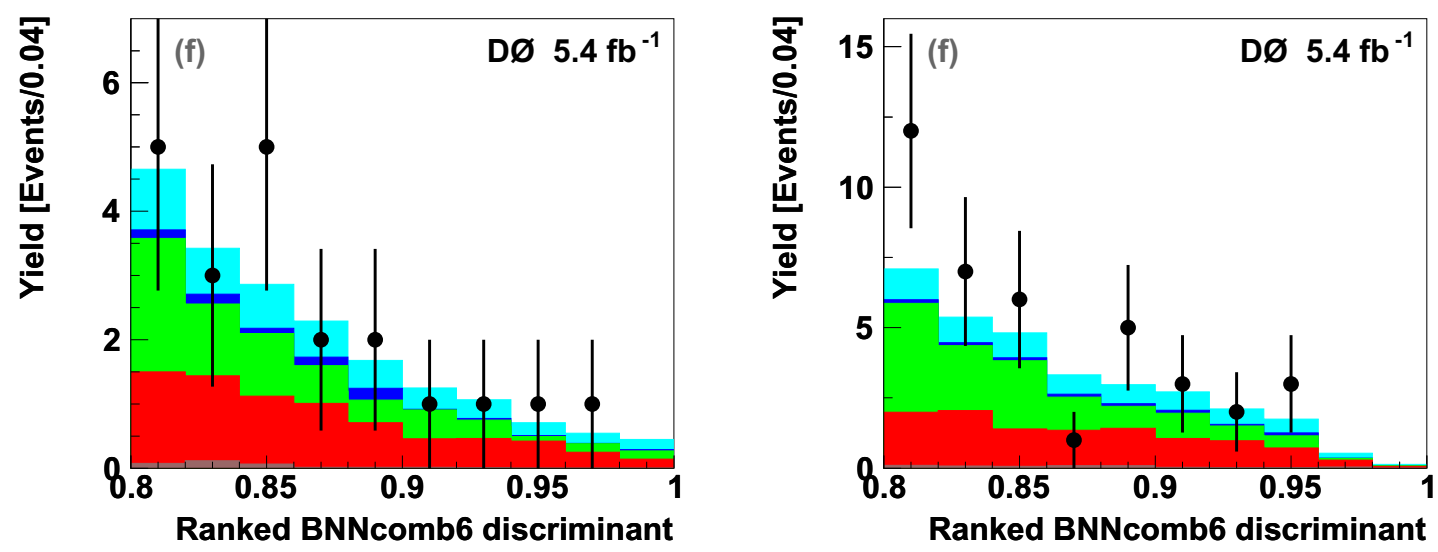

Figure C.6: The BNNcombSSortZoom plots in the electron+muon channel for positive (left) and negative (right) events. 


\section{Appendix D}

\section{Lepton Charge Mis-Identification}

The charge measurement is important for this analysis since $\mathrm{CP}$ violation can be diluted by the mis-identification (mis-ID) of the lepton charge. We rely on the track charge to determine whether the selected lepton carries positive or negative charge.

We select $Z \rightarrow e e$ or $Z \rightarrow \mu \mu$ events to measure the charge mis-ID rate. We require each event to have exactly two electrons or two muons, and the invariant mass of the leptons to be between 80 and $100 \mathrm{GeV}$ ( $Z$ peak). We assume that all same sign events (defined as the events containing two same sign leptons) under the $Z$ peak must have one lepton with the wrong charge, and that opposite sign events (defined as the events containing two opposite sign leptons) have correctly measured lepton charges. Therefore the charge mis-ID rate is defined as the ratio between the number of same sign events and the total number of di-lepton events. 
We measured the lepton charge mis-ID rate dependences on lepton detector eta and lepton transverse momentum using CSG 2EMhighpt and 2MUhighpt data samples. The luminosity of the data is $1.1 \mathrm{fb}^{-1}$ for p17 and $4.3 \mathrm{fb}^{-1}$ for p20.

We also measured the mis-ID rate from MC samples as a cross check.

The data and simulation samples used in this analysis are listed below. Electron Channel data samples:

- CSG_CAF_2EMhighpt_PASS3_p18.13.01 for RunIIa

- CSG_CAF_2EMhighpt_PASS4_p21.10.00_p20.12.00 for RunIIb

- CSG_CAF_2EMhighpt_PASS4_p21.10.00_p20.12.01 for RunIIb

- CSG_CAF_2EMhighpt_PASS4_p21.10.00_p20.12.02 for RunIIb

- CSG_CAF_2EMhighpt_PASS4_p21.10.00_p20.12.05 for RunIIb

- CSG_CAF_2EMhighpt_PASS2_p21.10.00 for RunIIb

Electron Channel MC samples:

- AFB-Zee-60-130GeV-p21 for RunIIb

- AFB-Zee-60-130GeV-NoExtraSmear for RunIIa

Muon Channel data samples:

- CSG_CAF_2MUhighpt_PASS3_p18.14.00 for RunIIa 
- CSG_CAF_2MUhighpt_PASS4_p21.10.00_p20.12.00 for RunIIb

- CSG_CAF_2MUhighpt_PASS4_p21.10.00_p20.12.01 for RunIIb

- CSG_CAF_2MUhighpt_PASS4_p21.10.00_p20.12.02 for RunIIb

- CSG_CAF_2MUhighpt_PASS4_p21.10.00_p20.12.03 for RunIIb

- CSG_CAF_2MUhighpt_PASS4_p21.10.00_p20.12.04 for RunIIb

- CSG_CAF_2MUhighpt_PASS4_p21.10.00_p20.12.05_summer2009 for RunIIb

- CSG_CAF_2MUhighpt_PASS2_p21.10.00 for RunIIb

Muon Channel MC samples:

- Zmumu-weigang-60-130GeV-p20_2 for RunIIb

- Zmumu-weigang-60-130GeV-p17 for RunIIa

For both channels we used the selection cuts as close as possible to the single top cross section measurement, except that we have 2 leptons instead of 1 (same isolation requirements for both leptons.), and we used the 2 jet inclusive bin while single top analysis uses 2, 3, 4 jet exclusive bins. Also there is no MET requirement. 


\section{D.1 Electron Charge Mis-ID Rate}

Electrons are defined as clusters of energy depositions in the electromagnetic section of the calorimeter, consistent in shape with an electromagnetic shower. We used these cuts for electrons in the charge mis-ID measurement:

- We require invariant mass $80<M_{12}<100 \mathrm{GeV}$

- We require electrons to be within the central calorimeter with $\left|\eta^{\text {det }}\right|<1.1$ (CC).

- electron transverse momentum $p_{T}>15 \mathrm{GeV}$

- $z$ (track, primaryvertex $)<1 \mathrm{~cm}$

- At lease $90 \%$ of the energy of the cluster must be contained in the electromagnetice section of the calorimeter. EMF $>90 \%$

- The isolation requirement is $\mathrm{ISO}<0.15$

- EM-likelihood $>0.85$

- $7 \times 7$ H-matrix $\chi^{2}<50$ HMx7cut $=50$.

- Track $p_{T}>5 \mathrm{GeV}$

- $\mathrm{RDCA}<2$. (= no rdca requirement.) 
- Track match changed from spatial match only to also EOP match included $($ track_match_spatialchi2prob ()$=>$ track_match_chi2prob ()$)$. This further lowers electron misid rate from 0.6 to 0.3 percent.

The mis-ID rate is less than $0.5 \%$ for both $\mathrm{MC}$ and data, in all bins. 


\section{D.2 Muon Charge Mis-ID Rate}

Muons are identified by combining tracks in the muon spectrometer $\left(\left|\eta^{\text {det }}\right|<2.0\right)$ with central detectors tracks. We used these cuts for muons in the charge mis-ID measurement:

- We require Invariant Mass $80<M_{12}<100 \mathrm{GeV}$

- We require muons to match the central calorimeter with $\left|\eta^{\text {det }}\right|<2.0$

- muon $p_{T}>15 \mathrm{GeV}$

- $z$ (track, primaryvertex $)<1 \mathrm{~cm}$

- We require muon not in a jet: $\Delta R($ muon, jet $)>0.5$

- Momenta of all tracks are within $R<0.5$ except muon track $<20 \%$ of muon pT: etTrkCone $5 / \mathrm{pT}<0.2$

- energy in cone $0.1<R<0.4$ less than $20 \%$ of muon pT: etHalo/pT $<0.2$

- pass 3 layers of muon scintilators: $\mid$ nseg $\mid=3$

- not from cosmic rays

- has a central track

- track Chi2Ndf()<4.0 
- when there is no SMT hits, require $D C A<0.2$ (track match muon in $\eta, \phi$.)

Figure D.1 shows the muon charge mis-ID rate as a function of lepton $p_{T}$ for Run IIa (left) and Run IIb (right).

The mis-ID rate is less than $1 \%$ for both MC and data, in all bins. 

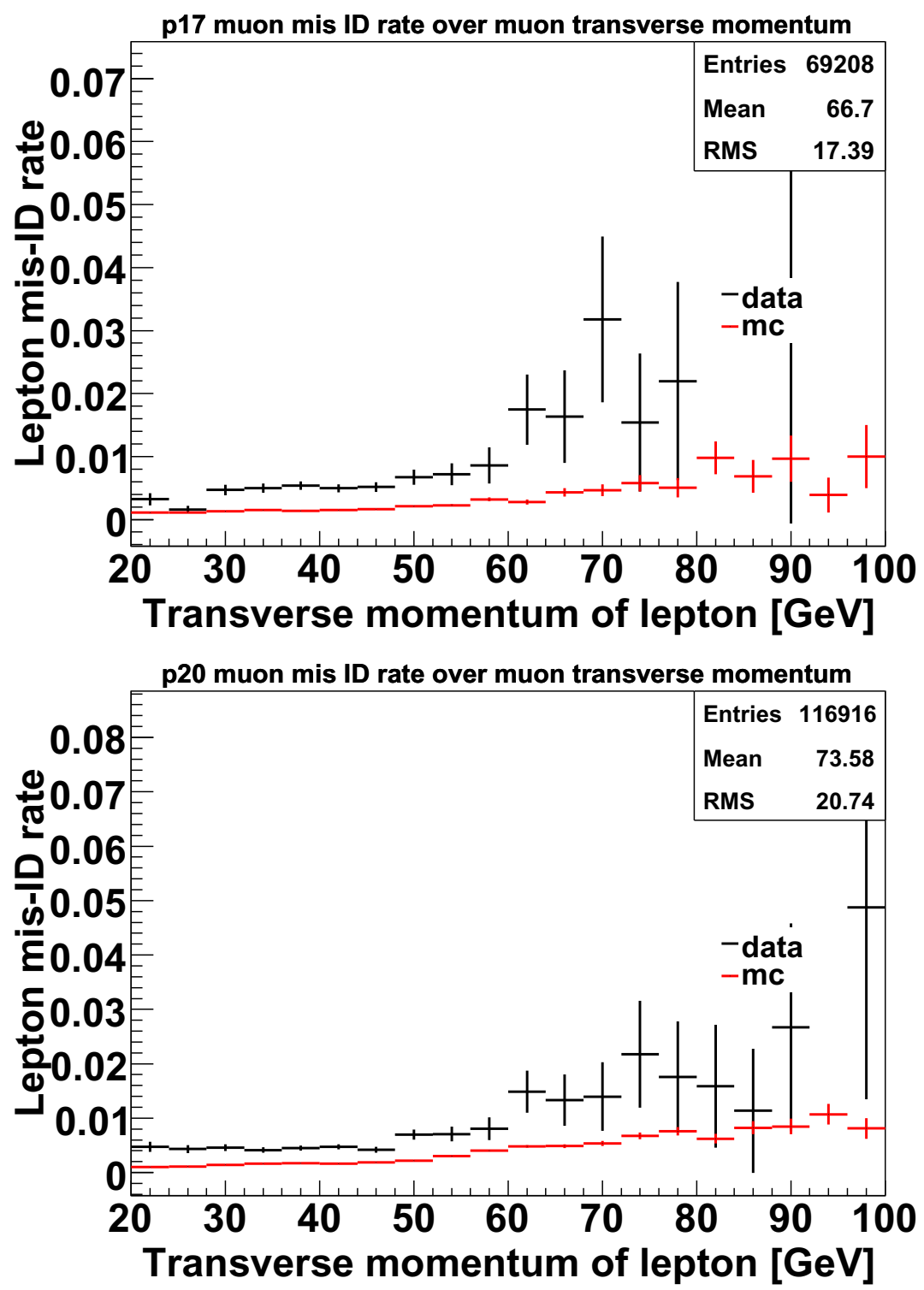

Figure D.1: The muon charge mis-ID rate as a function of lepton $p_{T}$ for Run IIa (left) and Run IIb (right). 


\section{D.3 Conclusion}

In both $\mathrm{MC}$ and data, the fake rate is always below 1\%, as shown in Table D.1. And since we are not sensitive to effects of the order of $1 \%$ compared to the existing much larger systematic uncertainties, we simply add a systematic uncertainty of $1 \%$ anticorrelated between the positive and negative charge samples. One may note that the data charge mis-ID rate is significantly higher than MC. This is because MC does not simulate our detector well. As long as they are all very small, it shouldn't affect the final result.

Table D.1: Overall Charge mis-ID Rates.

\begin{tabular}{c||cc|cc}
\multicolumn{1}{c||}{} & \multicolumn{2}{c}{ Overall charge mis-ID rates } \\
\multicolumn{1}{c|}{ Run IIa } & \multicolumn{2}{c}{ Run IIb } \\
& Electron & Muon & Electron & Muon \\
\hline Data & $0.22 \%(1 \pm 0.11)$ & $0.52 \%(1 \pm 0.053)$ & $0.32 \%(1 \pm 0.074)$ & $0.50 \%(1 \pm 0.041)$ \\
MC & $0.12 \%(1 \pm 0.036)$ & $0.15 \%(1 \pm 0.016)$ & $0.068 \%(1 \pm 0.054)$ & $0.18 \%(1 \pm 0.0068)$
\end{tabular}




\section{Appendix E}

\section{W Asymmetry Check}

We performed a cross-check, for measuring the $W^{+}$and $W^{-}$asymmetry in the $W+$ jets sample. This check was done by using $W+$ jets samples as the signal and tbtqb sample as a part of the backgrounds in the 2-jet and 1-tag bin. The expected and observed posterior density funcions are shown in Fig. E.1 to Fig. E.2, taking into account all systematics. The $W$ asymmetry is found to be consistent with 0 (as expected) and compared to the single top asymmetry we are trying to measure. This check is sensitive to possible bias from the detector effect, and we find that there are none. 


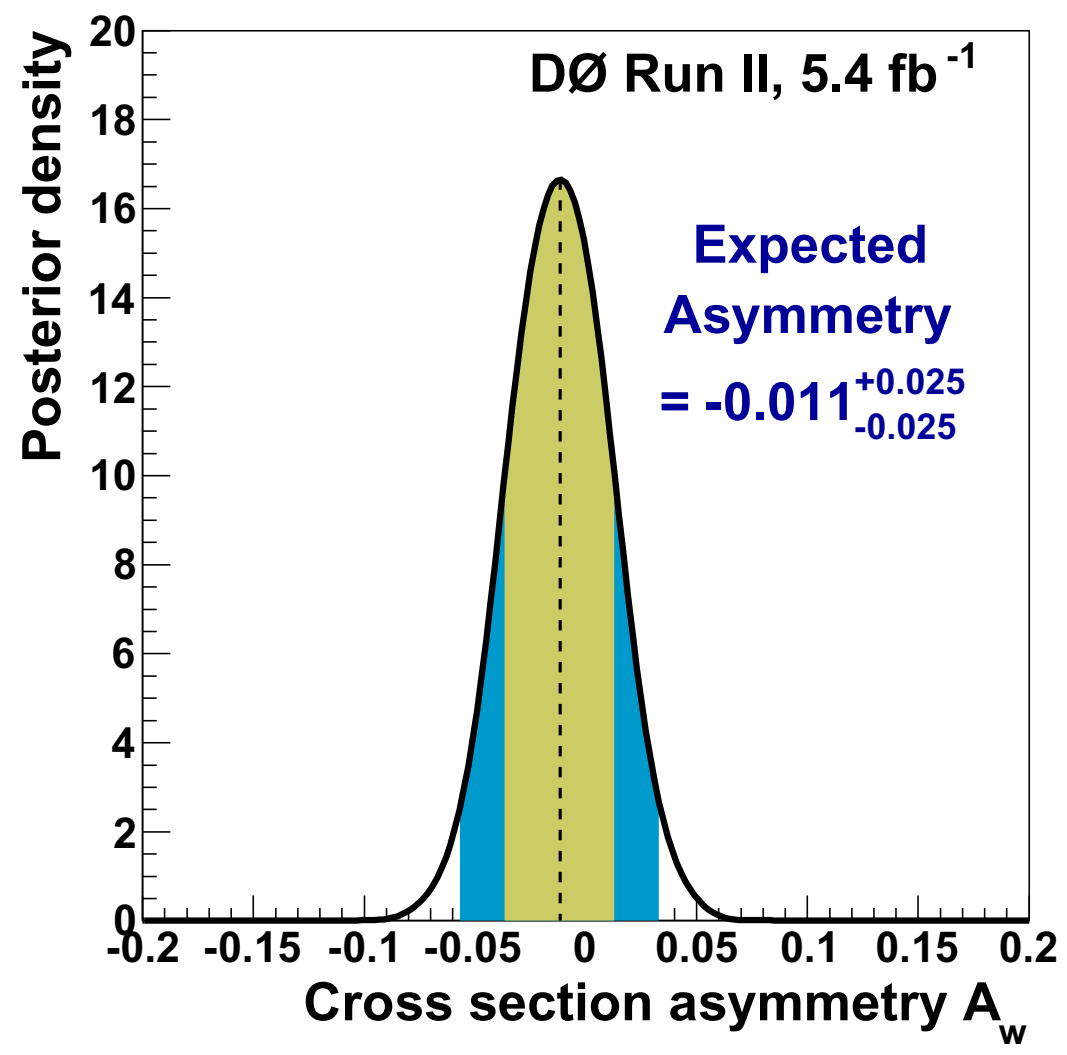

Figure E.1: Expected posterior density distributions for $W+$ jets asymmetry in the $s+t$-channel 2-jet 1-tag bin. 


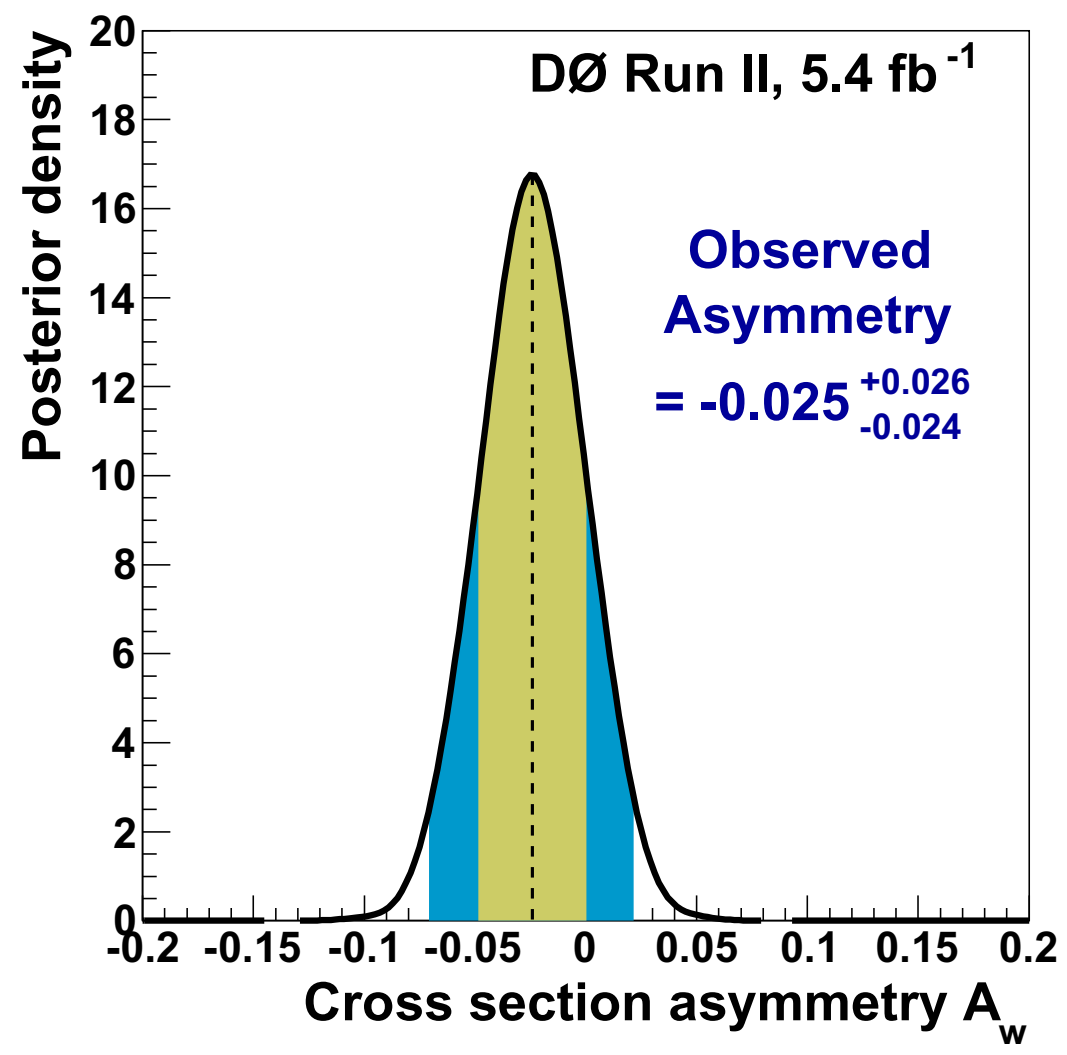

Figure E.2: Observed posterior density distributions for $W+$ jets asymmetry in the $s+t$-channel 2-jet 1-tag bin. 


\section{Appendix F}

\section{$b / \bar{b}$ Jet Energy Scale (JES) Check}

The measurement of the top-antitop cross section difference could be affected by a different response of the calorimeter to quark and antiquark jets. Such a bias could come from a different calorimeter response to the $b$ and the $\bar{b}$ decays, respectively. In principle, $c$ and $\bar{c}$ responses in the calorimeter could affect $b / \bar{b}$ JES also, but it's an even smaller effect, thus we only check $b$ and $\bar{b}$ responses check.

We rescale the pT (the 4 -vectors accordingly) of the $b$ and $\bar{b}$ by a factor of 0.9971 and 1.0021 respectively [73], depending on $b$ or $\bar{b}$, and then re-apply the MVA filters, for all MC samples. Then, we evaluate the difference in the $\mathrm{CP}$ violation extracted from the original and modified samples. We only do this for the signal, which we expect will have the largest effect, because our main measurements come from the signal region of the signal asymmetry posteriors. 
The Jet Energy Scale (JES) is applied before the event selection and can introduce changes not only to event yields, but also because the jet kinematics are changed. It can change the jet multiplicity distribution of the events, and thus affect the number of events passing the selection. Tables F.1 to F.4 shows the expected event yields for the signals after b-tagging before and after applying b/bbar JES for both "positive" and "negative" samples. We can see that within errors the event yields do not change. Table F.1: Number of expected yields for signals in "positive" samples after btagging.

\begin{tabular}{l|cccc} 
Source & 2 jets & 3 jets & 4 jets & All Channels \\
\hline$t b$ & $53 \pm 7.6$ & $22 \pm 3.7$ & $6.4 \pm 1.7$ & $81 \pm 13$ \\
$t q b$ & $70 \pm 5.6$ & $37 \pm 4.4$ & $13 \pm 2.9$ & $119 \pm 13$ \\
$t b+t q b$ & $122 \pm 13$ & $59 \pm 8.1$ & $19 \pm 4.6$ & $200 \pm 26$
\end{tabular}

Table F.2: Number of expected yields for signals in "positive" samples after b-tagging for b/bbar JES.

\begin{tabular}{l|cccc} 
Source & 2 jets & 3 jets & 4 jets & All Channels \\
\hline$t b$ & $53 \pm 0.84$ & $22 \pm 0.54$ & $6.5 \pm 0.26$ & $81 \pm 1.5$ \\
$t q b$ & $70 \pm 1.2$ & $36 \pm 0.95$ & $13 \pm 0.53$ & $120 \pm 2.4$ \\
$t b+t q b$ & $123 \pm 1.9$ & $58 \pm 1.4$ & $19 \pm 0.74$ & $200 \pm 3.9$
\end{tabular}

To check the normalization effects, we compare a few variables before and after 
Table F.3: Number of expected yields for signals in "negative" samples after btagging.

\begin{tabular}{l|cccc} 
Source & 2 jets & 3 jets & 4 jets & All Channels \\
\hline$t b$ & $52 \pm 7.6$ & $22 \pm 3.7$ & $6.5 \pm 1.6$ & $80 \pm 13$ \\
$t q b$ & $71 \pm 5.5$ & $36 \pm 4.2$ & $13 \pm 2.9$ & $120 \pm 13$ \\
$t b+t q b$ & $123 \pm 13$ & $58 \pm 7.9$ & $20 \pm 4.5$ & $200 \pm 25$
\end{tabular}

Table F.4: Number of expected yields for signals in "negative" samples after btagging for $\mathrm{b} / \mathrm{bbar}$ JES

\begin{tabular}{l|cccc} 
Source & 2 jets & 3 jets & 4 jets & All Channels \\
\hline$t b$ & $52 \pm 0.84$ & $22 \pm 0.54$ & $6.4 \pm 0.26$ & $80 \pm 1.5$ \\
$t q b$ & $71 \pm 1.2$ & $36 \pm 0.94$ & $13 \pm 0.52$ & $120 \pm 2.4$ \\
$t b+t q b$ & $123 \pm 1.9$ & $58 \pm 1.4$ & $19 \pm 0.73$ & $200 \pm 3.9$
\end{tabular}


$b / \bar{b}$ JES. To obtain a numerical estimate, we integrate from the peak bin, and look into the ratios of the integrals before and after this JES. This is done for $t b+t q b$ combined for the one tag two jet bin for positive and negative samples separately. We looked into several variables that are sensitive to this $b / \bar{b}$ JES: HT for all jets, Leading jet transverse momentum, Single top mass. The results are summarized in Table F.5. The plots are show in Figure F.1 to Figure F.6.

Table F.5: Ratio of integrals for several kinematic variables before and after $\mathrm{b} / \bar{b}$ JES: HT for all jets, Leading jet transverse momentum, Single top mass, in the positive and negative tbtqb samples in one tag two jet bin. The integral is from the peak bin.

\begin{tabular}{l|cc} 
& positive & negative \\
\hline HT $_{\text {All Jets }}$ & 0.987 & 0.993 \\
PT $_{\text {Jet } 1}$ & 0.986 & 0.994 \\
$M_{\text {top }}$ & 0.990 & 0.982
\end{tabular}

Based on these findings, we apply another 1\% anti-correlated flat uncertainty on single top and ttbar and Wbb samples, i.e. 0.01 for the top files and -0.01 for the antitop files. 


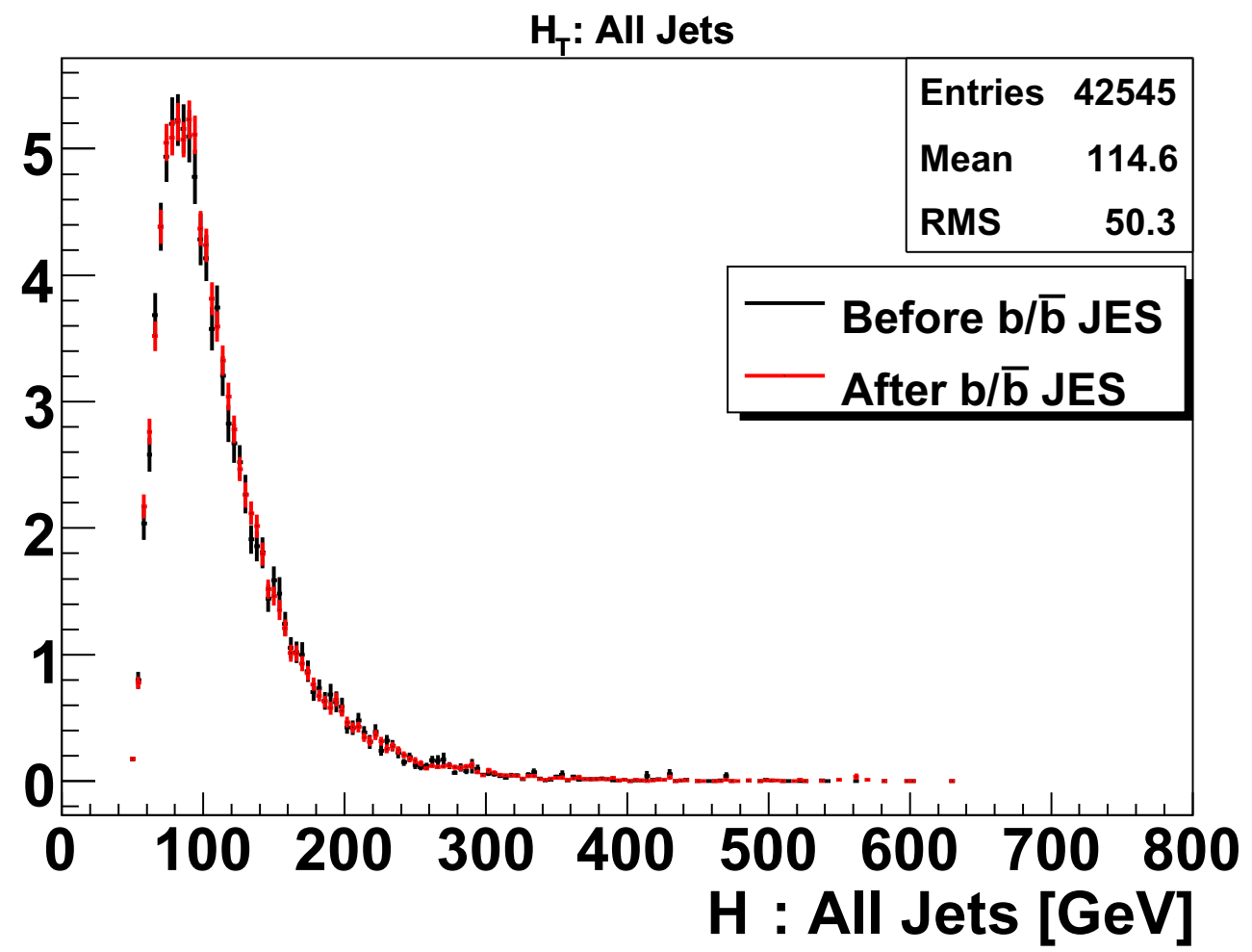

Figure F.1: HT for all jets before and after $\mathrm{b} / \bar{b}$ JES for positive tbtqb samples in one tag two jet bin. 


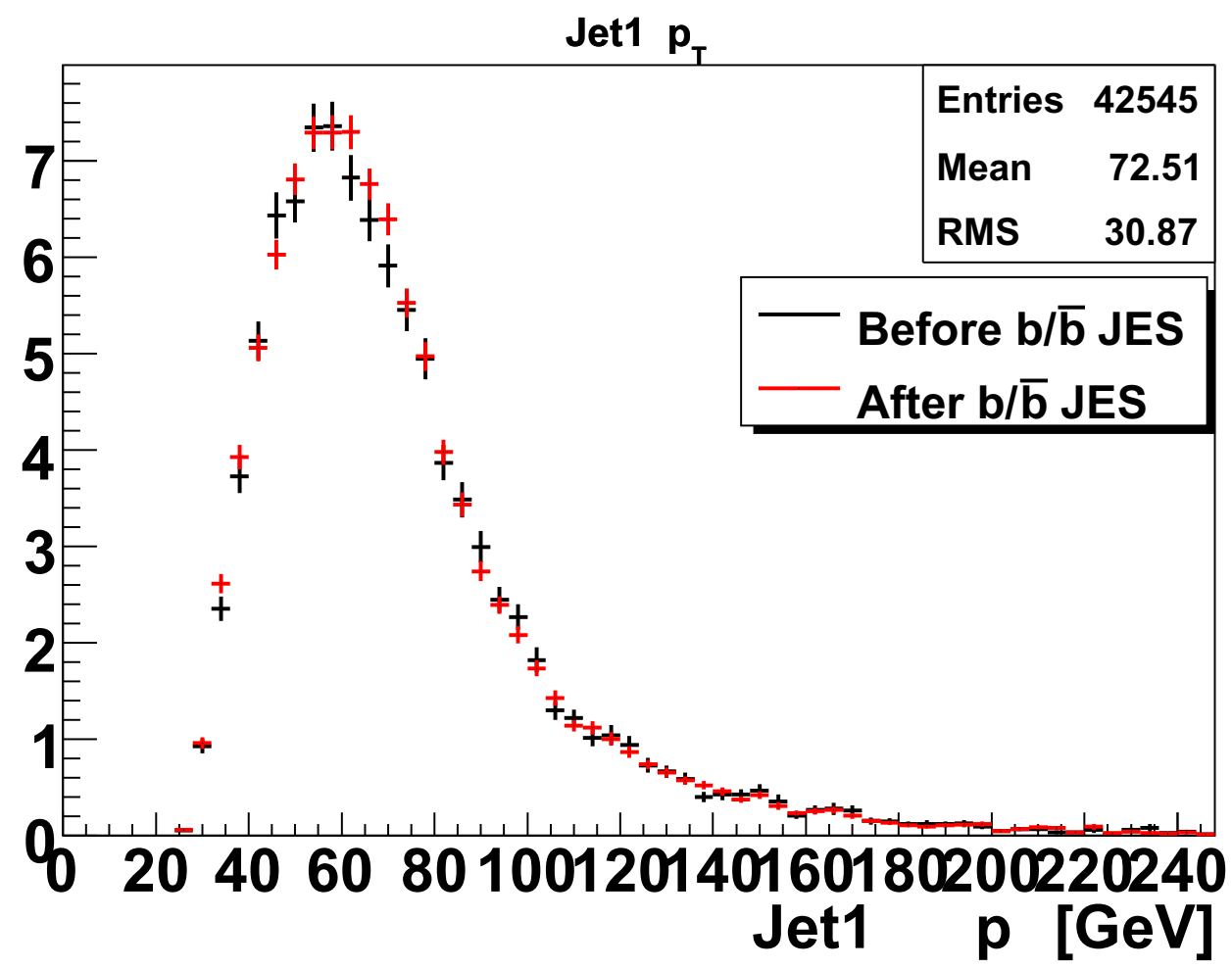

Figure F.2: Leading jet transverse momentum before and after $\mathrm{b} / \bar{b}$ JES for positive tbtqb samples in one tag two jet bin. 
Top mass corresponding to minimal significance

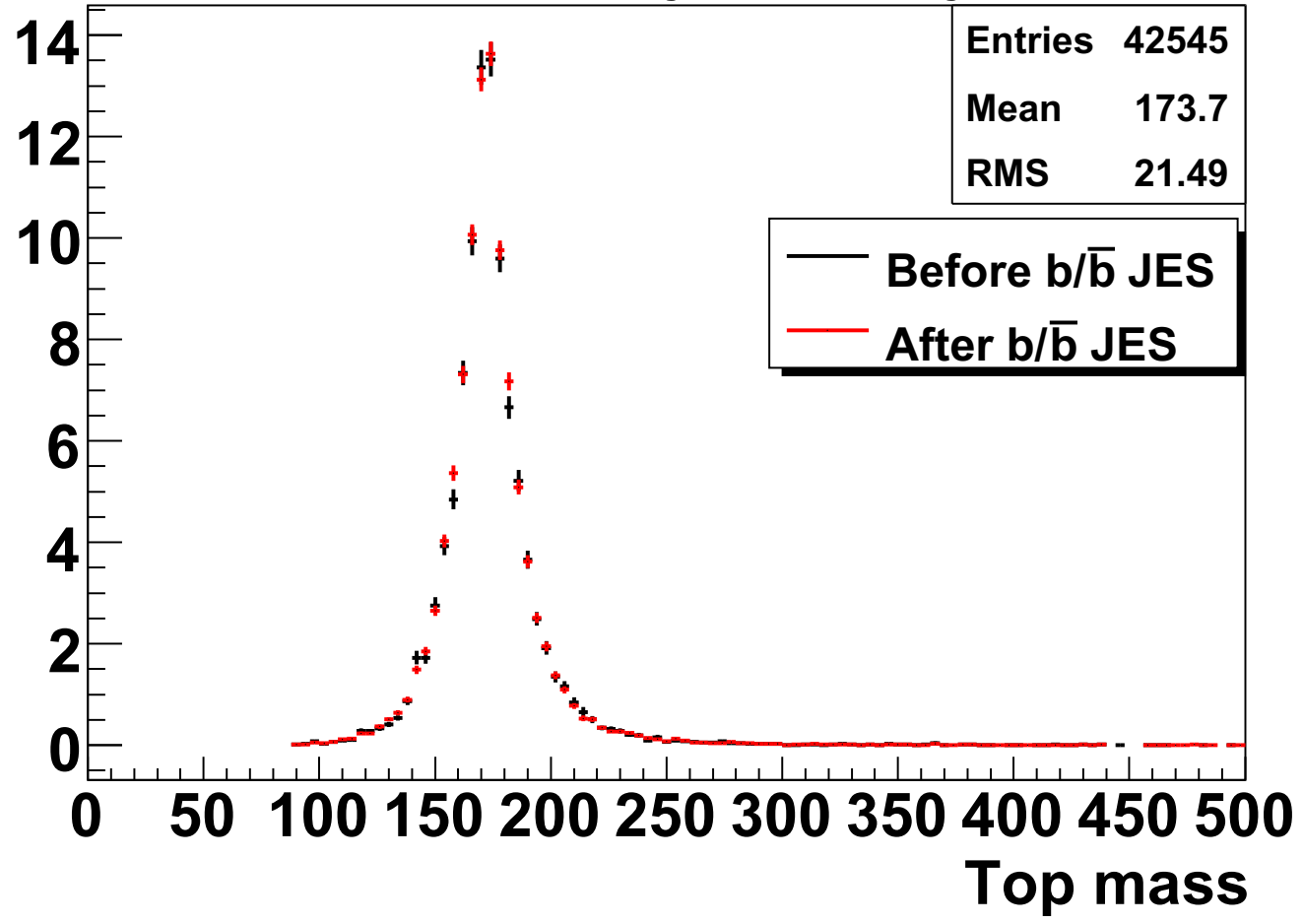

Figure F.3: Single top mass before and after b/ $\bar{b}$ JES for positive tbtqb samples in one tag two jet bin. 


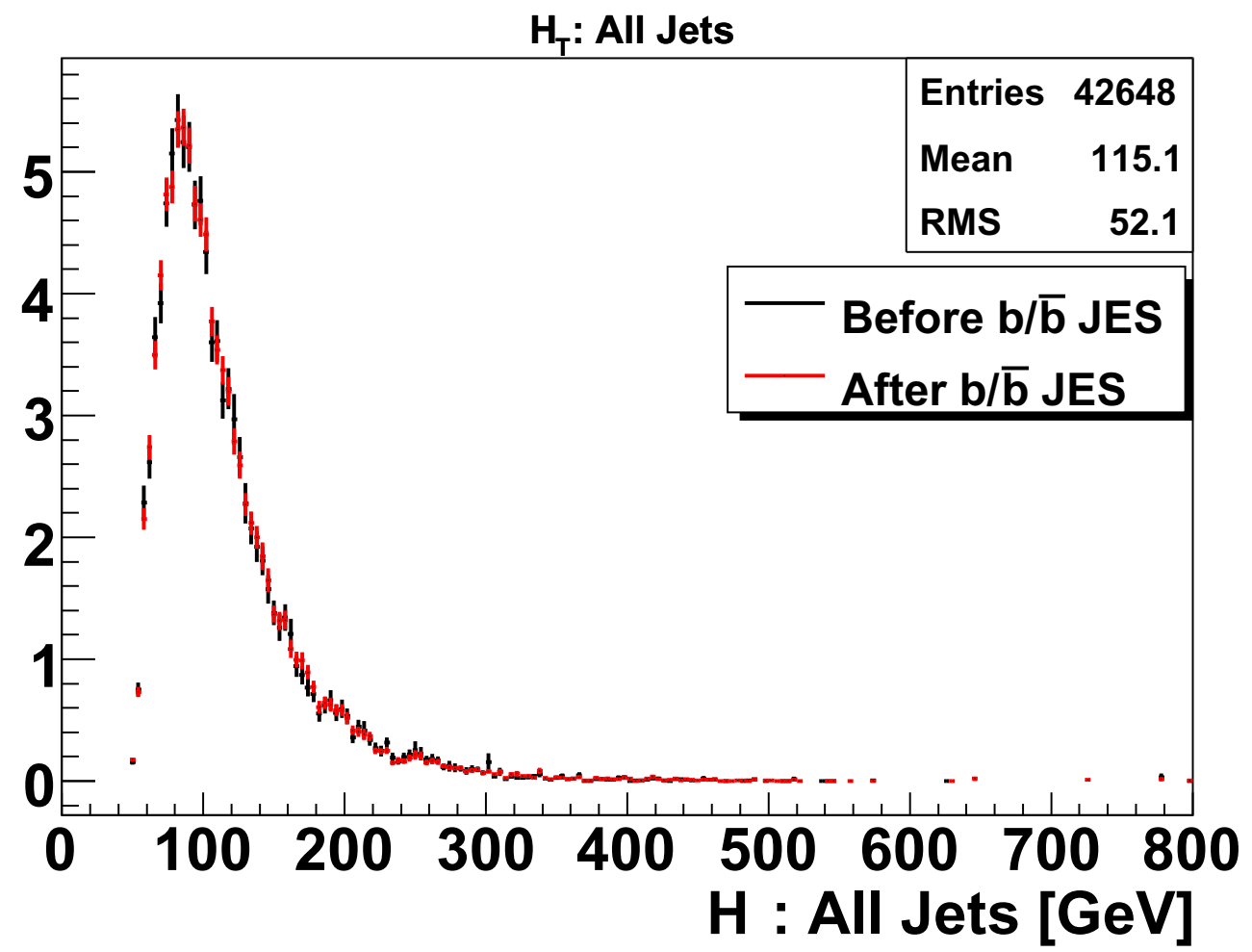

Figure F.4: HT for all jets before and after b/ $/ \bar{b}$ JES for negative tbtqb samples in one tag two jet bin. 


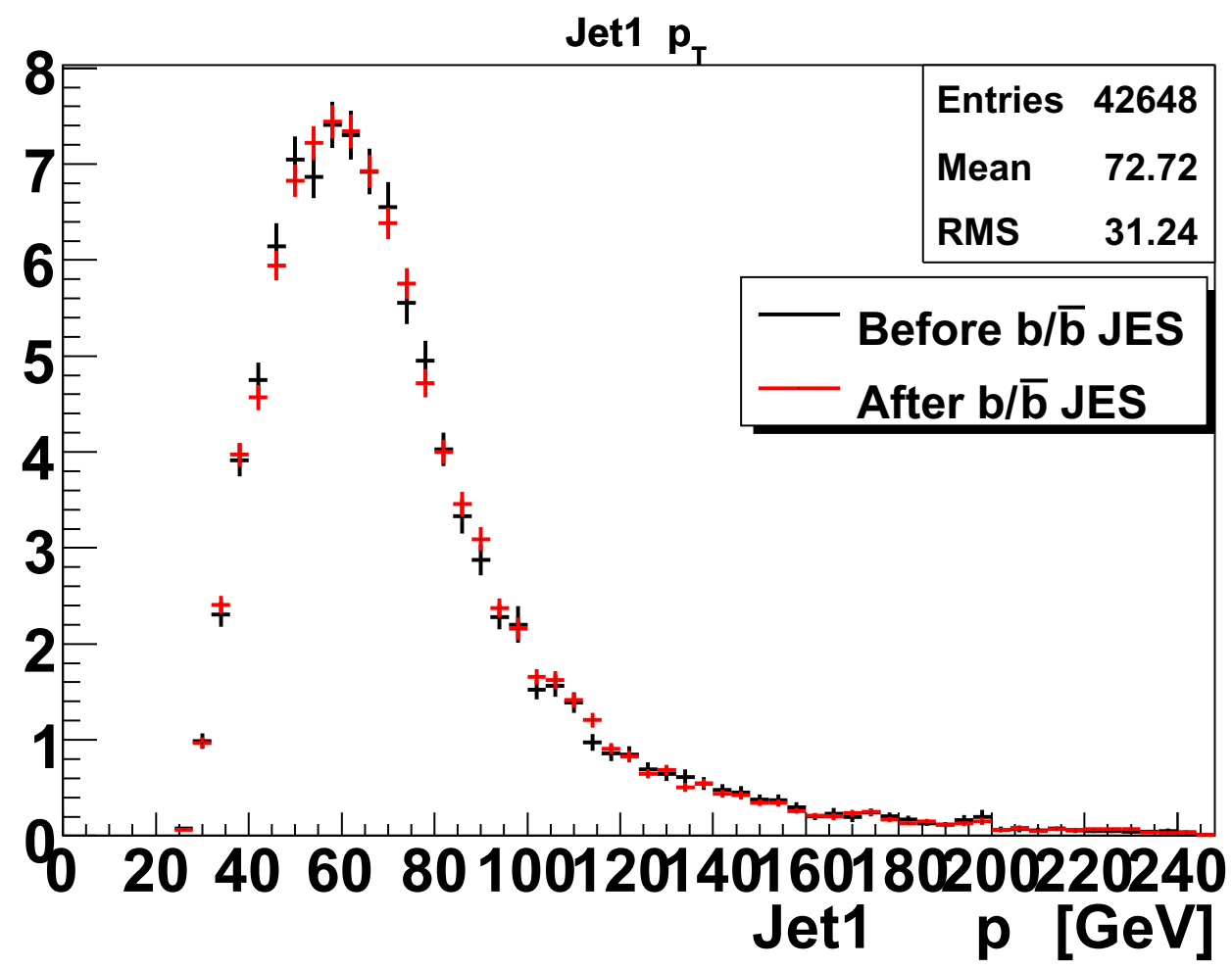

Figure F.5: Leading jet transverse momentum before and after $\mathrm{b} / \bar{b}$ JES for negative tbtqb samples in one tag two jet bin. 
Top mass corresponding to minimal significance

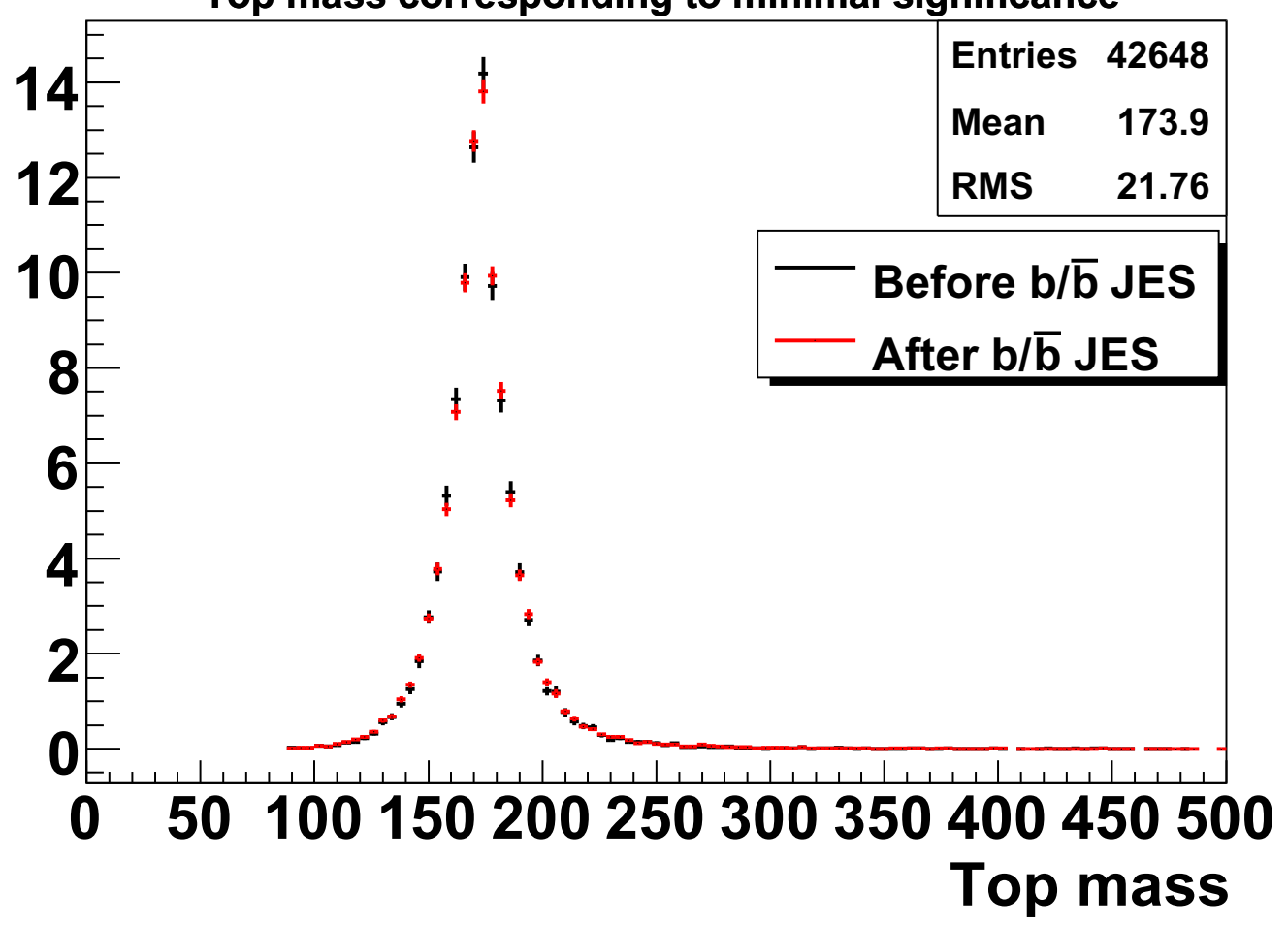

Figure F.6: Single top mass before and after b/ $\bar{b}$ JES for negative tbtqb samples in one tag two jet bin. 


\section{Appendix G}

\section{Production and decay asymmetries}

The asymmetry in single top production can be expressed as follows:

$$
\mathcal{A}_{P}=\frac{\sigma(p \bar{p} \rightarrow t X)-\sigma(p \bar{p} \rightarrow \bar{t} X)}{\sigma(p \bar{p} \rightarrow t X)+\sigma(p \bar{p} \rightarrow \bar{t} X)} .
$$

The asymmetry can also originate from the top decay $t \rightarrow b W^{+}$or $\bar{t} \rightarrow \bar{b} W^{-}$, quantified by the partial rate asymmetry:

$$
\mathcal{A}_{D}=\frac{\Gamma\left(t \rightarrow b W^{+}\right)-\Gamma\left(\bar{t} \rightarrow \bar{b} W^{-}\right)}{\Gamma\left(t \rightarrow b W^{+}\right)+\Gamma\left(\bar{t} \rightarrow \bar{b} W^{-}\right)}
$$

where $\Gamma$ denotes the decay rate.

It is customary when measuring a cross section to assume that the top quark decays exclusively in $t \rightarrow b W$ and ignore any possible $\mathrm{CP}$ violation contribution from 
the decay process. However, in the more general case, the measured asymmetry $\mathcal{A}$ is equal to

$$
\mathcal{A}=\frac{\sigma(p \bar{p} \rightarrow t X) \mathcal{B}\left(t \rightarrow b W^{+}\right)-\sigma(p \bar{p} \rightarrow \bar{t} X) \mathcal{B}\left(\bar{t} \rightarrow \bar{b} W^{-}\right)}{\sigma(p \bar{p} \rightarrow t X) \mathcal{B}\left(t \rightarrow b W^{+}\right)+\sigma(p \bar{p} \rightarrow \bar{t} X) \mathcal{B}\left(\bar{t} \rightarrow \bar{b} W^{-}\right)}
$$

where $\mathcal{B}$ denotes the branching ratio. Note the asymmetry defined in this way is always smaller than one.

The decay asymmetry can be written as a function of $\Gamma\left(t \rightarrow b W^{+}\right)=\Gamma_{t} \mathcal{B}(t \rightarrow$ $\left.b W^{+}\right)$and $\Gamma\left(\bar{t} \rightarrow \bar{b} W^{-}\right)=\Gamma_{\bar{t}} \mathcal{B}\left(\bar{t} \rightarrow \bar{b} W^{-}\right)$where $\Gamma_{t}$ and $\Gamma_{\bar{t}}$ are the total decay width for $t$ and $\bar{t}$ quark. Because of the conservation $\Gamma_{t}=\Gamma_{\bar{t}}=\Gamma$, the measured asymmetry can be expressed in terms of the partial decay rates

$$
\mathcal{A}=\frac{\sigma(p \bar{p} \rightarrow t X) \Gamma\left(t \rightarrow b W^{+}\right)-\sigma(p \bar{p} \rightarrow \bar{t} X) \Gamma\left(\bar{t} \rightarrow \bar{b} W^{-}\right)}{\sigma(p \bar{p} \rightarrow t X) \Gamma\left(t \rightarrow b W^{+}\right)+\sigma(p \bar{p} \rightarrow \bar{t} X) \Gamma\left(\bar{t} \rightarrow \bar{b} W^{-}\right)}
$$

Therefore the measured asymmetry contains contributions from both production and partial rate asymmetries. In particular, it can be shown that

$$
\mathcal{A}=\frac{\mathcal{A}_{P}+\mathcal{A}_{D}}{1+\mathcal{A}_{P} \mathcal{A}_{D}}
$$

If one assumes that $\mathrm{CP}$ violation only happens in the top production or $\mathcal{A}_{D}$ is negligible [5], then we have $\mathcal{A}_{D} \approx 0, \mathcal{A}=\mathcal{A}_{P}$. On the other hand, if one assumes 
that $\mathcal{A}_{D}=\mathcal{A}_{P}$, then $\mathcal{A}=\frac{2 \mathcal{A}_{P}}{1+\mathcal{A}_{P}^{2}}$. In this analysis, we only measure the asymmetry $\mathcal{A}$ 
BIBLIOGRAPHY 


\section{BIBLIOGRAPHY}

[1] N. Kidonakis. Single top quark production at the tevatron: threshold resummation and finite-order soft gluon corrections. Phys. Rev. D, 74:114012, 2006.

[2] L. D. Landau. On the Conservation Laws in Weak Interactions . Zh. Eksp. Teor. Fiz., 32:405, 1957.

[3] V.L. Fitch J. Christenson, J.W. Cronin and R. Turlay. Evidence for the $2 \pi$ Decay of the K20 Meson. Phys. Rev. Lett., 13:138.

[4] S. Abachi et al. Observation of the top quark. Phys. Rev. Lett., 74:2632, 1995.

[5] D Atwood S. Bar-Shalom and A. Soni. CP Violation in Single Top Production and Decay Via $p b a r p \rightarrow t b a r b+X \rightarrow W^{+} b \bar{b}+X$ Within the MSSM: A possible Application for Measuring $\arg \left(A_{t}\right)$ at Hadron Colliders . Phys. Rev. D, 57:1495.

[6] G. Eilan A. Soni D. Atwood, S. Bar-Shalom. CP nonconservation in $p \bar{p} \rightarrow t b X$ at the Fermilab Tevatron. Phys. Rev. D, 54:5412.

[7] Makoto Kobayashi and Toshihide Maskawa. CP Violation in the Renormalizable Theory of Weak Interaction. Prog. Theor. Phys., 49:652-657, 1973.

[8] G. Eilan A. Soni D. Atwood, S. Bar-Shalom. CP Violation in Top Physics . Phys. Rept., 1:347.

[9] S. F. Novaes. Standard model: An Introduction. hep-ph/0001283, 1999. 
[10] V. D. Barger and R. J. N. Phillips. Collider Physics (Frontiers in Physics). Addison Wesley Publishing Company, 1987.

[11] D. Griffiths. Introduction to elementary particles. Weinheim, USA: Wiley-VCH, 1987.

[12] M. Kaku. Quantum Field Theory, a modern introduction. Oxford University Press, 1993.

[13] C.-P. Yuan. A New Method to Detect a Heavy Top Quark at the Tevatron. Phys. Rev., D41:42, 1990.

[14] S. Willenbrock and D. Dicus. Production of Heavy Quarks from W Gluon Fusion. Phys. Rev., D34:155, 1986.

[15] V. M. Abazov et al. Observation of Single Top Quark Production. arXiv:0903.0850, 2009.

[16] T. Aaltonen et al. First Observation of Electroweak Single Top Quark Production. arXiv:0903.0885, 2009.

[17] Wikipedia. Standard model — wikipedia, the free encyclopedia, 2009. [Online; accessed 8-July-2009].

[18] W. Pauli. Über den Zusammenhang des Abschlusses der Elektronengruppen im Atom mit der Komplexstruktur der Spektren (¿On the Connexion between the Completion of Electron Groups in an Atom with the Complex Structure of Spectrai) . Z. Phys., 31:765, 1925.

[19] R. Brout F. Englert. Broken Symmetry and the Mass of Gauge Vector Mesons. Phys. Rev. Lett., 13(9):321-323, 1964.

[20] P. W. Higgs. Broken Symmetries and the Masses of Gauge Bosons. Phys. Rev. Lett., 13(16):508-509, 1964. 
[21] T.W.B. Kibble G.S. Guralnik, C.R. Hagen. Global Conservation Laws and Massless Particles. Phys. Rev. Lett., 13(20):585-587, 1964.

[22] NASA. Seven-year wilson microwave anisotropy probe (wmap) observations: Sky maps, systematic errors, and basic results, 2010. [nasa.gov. Retrieved 201012-02.].

[23] F. P. An. Observation of electron-antineutrino disappearance at Daya Bay. arXiv:1203.1669v2, 2012.

[24] B. A. Brak. The Hierarchy Problem in the Standard Model and Little Higgs Theories (Chapter 5). 2004.

[25] V. Trimble. Existence and nature of dark matter in the universe. Annual Review of Astronomy and Astrophysics, 25:425-472, 1987.

[26] C. Amsler et al. Review of particle physics. Phys. Lett., B667:1, 2008.

[27] F. Maltoni F. Tramontano J. M. Campbell, R. Frederix. NLO predictions for t-channel production of single top and fourth generation quarks at hadron colliders. arXiv:0907.3933, 2009.

[28] C.-P. Yuan. Strategies for Probing CP Properties in the Top Quark System at $e^{-} e^{+}$and Hadron Colliders . Mod. Phys. Lett. A, 10:627.

[29] The Single Top Working Group. Single top quark production in $5.4 \mathrm{fb}^{-1}$ of data - signal and background modeling and event selection. DØ Note, 6099, 2010 .

[30] The Single Top Working Group. Measurement of the single top quark production cross section in $5.4 \mathrm{fb}^{-1}$ of data. DØ Note, $\mathbf{6 1 2 8}, 2010$.

[31] (Ed. ) F.T. Cole, (Ed. ) E.L. Goldwasser, and (Ed. ) R.R. Wilson. National Accelerator Laboratory design report January 1968. 1968. FERMILAB-DESIGN1968-01. 
[32] Fermilab National Accelerator Laboratory, 2008. http://www.fnal.gov.

[33] Pushpalatha C. Bhat and William J. Spalding. Fermilab collider Run II: Accelerator status and upgrades. AIP Conf. Proc., 753:30-41, 2005.

[34] Fermilab. Tevatron luminosity, 2002. [Online; accessed 28-January-2012].

[35] Wikimedia Commons. A schematic of the basic components of a hermetic detector, 2012. http://upload.wikimedia.org/wikipedia/commons/e/eb/Hermetic.png.

[36] T. Han. Collider Phenomenology - Basic Knowledge and Techniques. 2005.

[37] G-Links Co. G-links, 2012. http://www.g-links.com.tw/.

[38] Cypress Semiconductor Corporation. Cypress hotlinks, 2012. http://www.cypress.com/.

[39] Fermilab National Accelerator Laboratory. D algorithms group. how to run reco, $2008 . \quad$ http://wwwd0.fnal.gov/computing/algorithms/howto/howtoreco.html.

[40] Wikipedia. Hough transform, 2012. [Online; accessed February-2012].

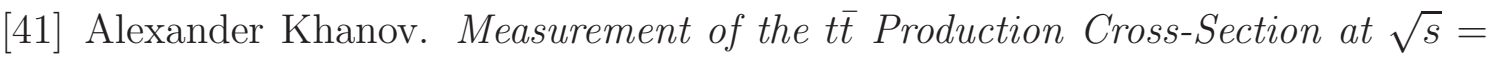
1.96 TeVUsingLifetimeTagging. PhD thesis, University of Rochester, 2004.

[42] G. Borissov. Ordering a chaos or .. technical details of aa tracking All DØ Meeting. 2003. http://www-d0.fnal.gov/atwork/adm/d0_private/2003-0228/adm_talk.ps.

[43] A. Schwartzman and C. Tully. Primary vertex reconstruction by means of adaptive vertex fitting. DØ Note, 4918, 2005. 
[44] Preliminary p17 jes for data and me (jetcorr v07-01-02), 2004. http://wwwd0.fnal.gov/phys_id/jes/d0_private/certified/certified_jes.html.

[45] N. Makovec and J.-F. Grivaz. Shifting, smearing and removing simulated jets. DØ Note, 4914, 2005.

[46] A. Harel and J. Kvita. p20 jetid efficiencies and scale factors. DØ Note, 5634, 2008.

[47] M. Anastasoaie, S. Robinson, and T. Scanlon. Performance of the nn $b$-tagging tool on p17 data. DØ Note, 5213, 2006.

[48] E. Barberis et. al. The matrix method and its error calculation. DØ Note, 4564, 2004.

[49] E. Boos, V. Bunichev, L. Dudko, V. Savrin, and V. Sherstnev. Method for simulating electroweak top-quark production events in the nlo approximation: Singletop event generator. Physics of Atomic Nuclei, 69(8):1317, 2006.

[50] J. Pumplin et. al. New generation of parton distributions with uncertainties from global QCD analysis. J. High Energy Physics, 07:012, 2002.

[51] T. Sjostrand, S. Mrenna, and P. Skands. PYTHIA 6.4 Physics and Manual. J. High Energy Physics, 05:026, 2006.

[52] D.J. Lange. The EvtGen particle decay simulation package. Nucl. Instrum. Meth., A462:152-155, 2001.

[53] M. Mangano, M. Moretti, F. Piccinini, R. Pittau, and A.D. Polosa. ALPGEN, a generator for hard multiparton processes in hadronic collisions. J. High Energy Physics, 07:001, 2003.

[54] J. Hays et al. Single electron efficiencies in p17 data and monte-carlo using p18.05.00 d0correct. DØ Note, 5105, 2006. 
[55] J. Atramentov et al. Electron and photon identification with p20 data. DØ Note, $5761,2008$.

[56] P. Calfayan et al. Muon identification certification for p17 data. DØ Note, $\mathbf{5 1 5 7 ,} 2006$.

[57] S. Muanza. A compilation of mcfm v5.1 cross section. DØ Note, 5406, 2007.

[58] The Single Top Working Group. Observation of single top quark production in $2.3 \mathrm{fb}^{-} 1$ of data using boosted decision trees. DØ Note, 5811, 2009.

[59] Y. Freund and R.E. Schapire. Experiments with a new boosting algorithm. Machine Learning: Proceedings of the Thirteenth International Conference, :148, 1996.

[60] B. A. Berg. Markov Chain Monte Carlo Simulations and Their Statistical Analysis. World Scientific Singapore, 2004.

[61] B. J. Pendleton S. Duane, A. D. Kennedy and D. Roweth. The boosting approach to machine learning an overview. MSRI Workshop on Nonlinear Estimation and Classification, 2002, 2002.

[62] The Single Top Working Group. Measurement of the single top quark production cross section in $5.4 \mathrm{fb}^{-} 1$ of data. DØ Note, 6128, 2011.

[63] R. Schwienhorst et. al. Statistical methods implemented in the package top_statistics. DØ Note, 5817, 2008.

[64] S. Moch and journal $=\mathrm{PRD}$ volume $=78$ pages $=034003$ year $=2008 \mathrm{P}$. Uwer, title $=$ Theoretical status and prospects for top-quark pair production at hadron colliders.

[65] F. Deliot et. al. $\mathrm{Z} / \gamma^{*}$ cross section computation at nnlo using the fewz code. DØ Note, 6050, 2010. 
[66] T. Nunnemann. The mcfm (n)nlo calculations of the diboson cross sections and their uncertainties, MCFM version 3.4.5. http://wwwclued0.fnal.gov/ nunne/cross-sections/mcfm_cross-sections.html.

[67] H. Schellman. The longitudinal shape of the luminous region at DØ. DØ Note, $\mathbf{5 1 4 2 ,} 2006$.

[68] H. Schellman. Run iib longitudinal beam shape. DØ Note, 5540, 2007.

[69] F. Deliot et. al. Systematic uncertainties in top quark measurements. DØ Note, $6024,2010$.

[70] Y. Peters, M. Begel, K. Hamacher, , and D. Wicke. Reweighting of the fragmentation function for the D $\varnothing$ monte carlo. DØ Note, 5325, 2007.

[71] V.M. Abazov et al. Measurement of the $t$ anti-t production cross section in $\mathrm{p}$ anti-p collisions at $\mathrm{s}^{* *}(1 / 2)=1.96$-tev using kinematic characteristics of lepton + jets events. Phys. Lett. B, 626:45, 2005.

[72] O. Brandt et. al. Muon identification certification for the summer 2009 extended dataset (run iib-1 and -2). DØ Note, 6109, 2010.

[73] O. Brandt et. al. Measurement of the mass difference between top and antitop quarks in the lepton+jets channel using the matrix element method on $3.6 \mathrm{fb}-1$ of run iib data. DØ Note, 6105, 2011. 